Modeled Neutron and Charged-Particle Induced Nuclear Reaction Cross Sections for Radiochemistry in the Region of Yttrium, Zirconium, Niobium, and Molybdenum

R. D. Hoffman, K. Kelley, F. S. Dietrich, R. Bauer, M. G. Mustafa

June 20, 2006 
This document was prepared as an account of work sponsored by an agency of the United States Government. Neither the United States Government nor the University of California nor any of their employees, makes any warranty, express or implied, or assumes any legal liability or responsibility for the accuracy, completeness, or usefulness of any information, apparatus, product, or process disclosed, or represents that its use would not infringe privately owned rights. Reference herein to any specific commercial product, process, or service by trade name, trademark, manufacturer, or otherwise, does not necessarily constitute or imply its endorsement, recommendation, or favoring by the United States Government or the University of California. The views and opinions of authors expressed herein do not necessarily state or reflect those of the United States Government or the University of California, and shall not be used for advertising or product endorsement purposes.

This work was performed under the auspices of the U.S. Department of Energy by University of California, Lawrence Livermore National Laboratory under Contract W-7405-Eng-48. 
UCRL-TR-222275

\title{
Modeled Neutron and Charged-Particle Induced Nuclear Reaction Cross Sections for Radiochemistry in the region of Yttrium, Zirconium, Niobium, and Molybdenum
}

\author{
R.D. Hoffman, K. Kelley, F. S. Dietrich and R. Bauer \\ Nuclear Theory and Modeling Group \\ Physics and Advanced Technologies, N-Division \\ Lawrence Livermore National Laboratory \\ Livermore, CA 94550 \\ rdhoffman@llnl.gov \\ M. G. Mustafa \\ Nuclear and Defense Technologies, AX-Division \\ Lawrence Livermore National Laboratory \\ Livermore, CA 94550
}

(June 20, 2006)

\begin{abstract}
We have developed a set of modeled nuclear reaction cross sections for use in radiochemical diagnostics. Systematics for the input parameters required by the Hauser-Feshbach statistical model were developed and used to calculate neutron, proton, and deuteron induced nuclear reaction cross sections for targets ranging from strontium $(Z=38)$ to rhodium $(Z=45)$.
\end{abstract}

Subject headings: Nuclear cross sections, Radiochemistry, Nuclear Physics

\section{Introduction}

\subsection{Radiochemistry}

Various aspects of nuclear explosive device performance can be determined through the use of radiochemistry. During the UGT (Under Ground Test) Program, select naturally occurring elements were often loaded into a device prior to a test and their activation products subsequently retrieved for counting. The products are measured as isotopic ratios (such as ${ }^{87} \mathrm{Y} /{ }^{88} \mathrm{Y}$ produced from a stable isotope of the naturally occurring element). From the measured activity and prior knowledge of the amount of loaded detector material, performance aspects could be inferred by comparing the measured isotope ratios with those calculated us- ing particle fluences from one of the design codes and group-averaged cross section sets that have been prepared for this purpose.

This paper continues the collaborative effort between AX-Division (DNT) and N-Division (PAT) to update and improve the existing RADCHEM cross section detector sets. Previous papers treated the regions of bromine and krypton (Hoffman et al. 2004a), iodine and xenon (Hoffman et al. 2004b), samarium, europium, and gadolinium (Hoffman et al. 2004c), scandium, titanium, vanadium, chromium, manganese, and iron (Kelley et al. 2005), arsenic (Kelley et al. 2006a), and nickel, copper, and zinc (Kelley et al. 2006b). Here we focus on reactions proceeding on targets of yttrium, zirconium, niobium, and molybdenum. 


\section{Contents}

1 Introduction $\quad 1$

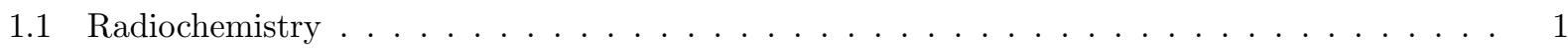

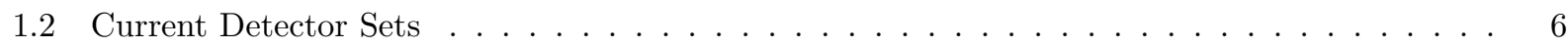

1.3 Motivation for Updating the Detector Sets . . . . . . . . . . . . . . . . . . . . 6

1.4 Proposed Detector Sets $\ldots \ldots \ldots \ldots \ldots \ldots \ldots$

$\begin{array}{lll}2 & \text { Nuclear Reaction Theory } & \mathbf{7}\end{array}$

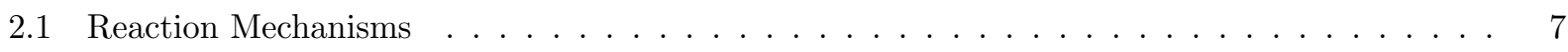

2.2 Hauser-Feshbach Statistical Model . . . . . . . . . . . . . . . . . . 7

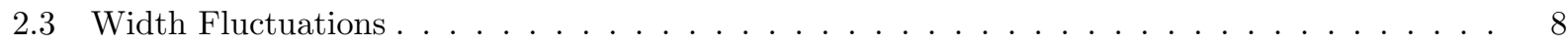

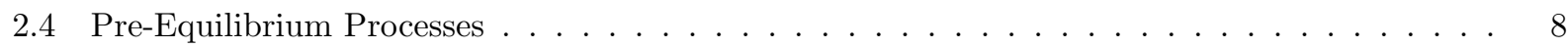

2.5 The STAPRE Hauser-Feshbach Reaction Code . . . . . . . . . . . . . . . . 9

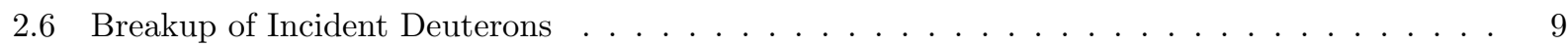

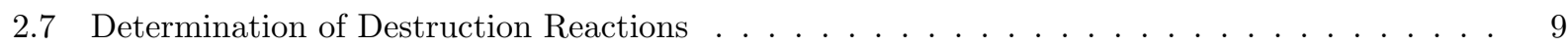

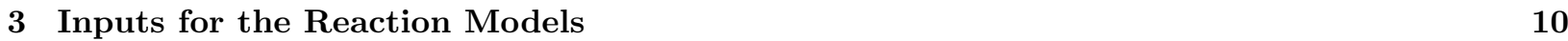

3.1 Nuclear Structure Data . . . . . . . . . . . . . . . . . . . . . . . . . . . . . . 10

3.1 .1 Ground State Masses and $J^{\pi}$ Assignments . . . . . . . . . . . . . . . 10

3.1 .2 Nuclear Level Schemes . . . . . . . . . . . . . . . . . . . . . . . . . 10

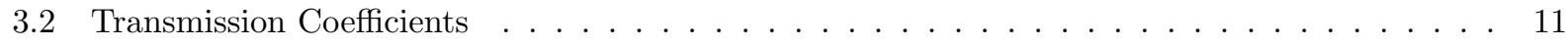

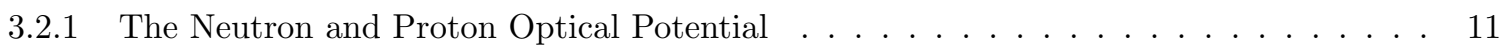

3.2 .2 The Alpha and Deuteron Optical Potentials . . . . . . . . . . . . . . . . . . 14

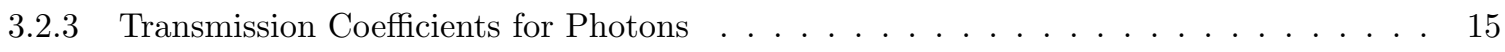

3.3 Nuclear Level Densities _. . . . . . . . . . . . . . . . . . . . . . . . 17

3.3 .1 Level Density Models . . . . . . . . . . . . . . . . . . . . 17

3.3 .2 Fermi-Gas Level Densities . . . . . . . . . . . . . . . . . . . . 18

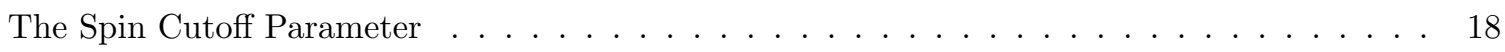

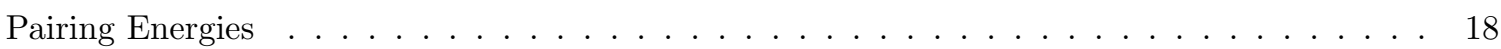

The Level Density Parameter . . . . . . . . . . . . . . . . . . . . . . . 19

Shell Corrections . . . . . . . . . . . . . . . . . . . . . . . 19

3.3.3 Constant Temperature Level Density . . . . . . . . . . . . . . . . . . . . . 19

Behavior of the Spin Cutoff Parameter Below $E_{x} \ldots \ldots \ldots \ldots \ldots \ldots$

3.4 Pre-Equilibrium Model Parameters . . . . . . . . . . . . . . . . . . . 22

4 Modeled Cross Sections $\quad 22$

4.1 Comparison to Measured Cross Sections . . . . . . . . . . . . . . . . . . 22

4.1.1 Comparison to experimental $(\mathrm{n}, \gamma)$ capture cross sections . . . . . . . . . . . 22

4.1.2 Comparison to Maxwellian averaged $(\mathrm{n}, \gamma)$ capture cross sections . . . . . . . . . . 23

4.1 .3 Comparison to experimental $(\mathrm{n}, 2 \mathrm{n})$ cross sections $\ldots \ldots \ldots \ldots \ldots \ldots$

4.1.4 Comparison to experimental $(\mathrm{n}, \mathrm{p})$ cross sections $\ldots \ldots \ldots \ldots \ldots \ldots$

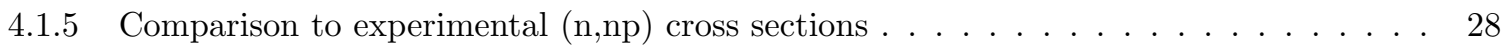

4.1.6 Comparison to experimental $(\mathrm{n}, \alpha)$ cross sections $\ldots \ldots \ldots \ldots \ldots \ldots$

4.1.7 Comparison to other experimental neutron induced cross sections . . . . . . . . . . . . 31

4.1.8 Comparison to experimental $(\mathrm{p}, \mathrm{n})$ cross sections $\ldots \ldots \ldots \ldots \ldots \ldots \ldots$

4.1.9 Comparison to other experimental charged particle cross sections . . . . . . . . . . 32 


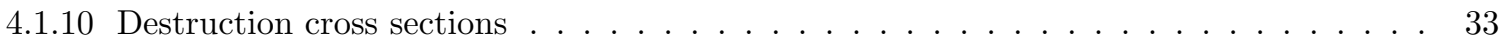

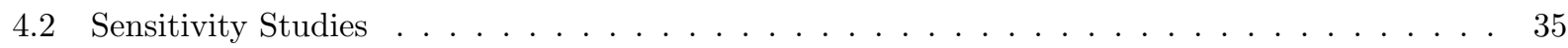

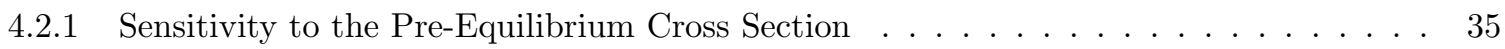

4.2 .2 Sensitivity to the Alpha Preformation Parameter . . . . . . . . . . . . . . 36

4.2.3 Sensitivity to the Level Density - Shell Correction Systematic . . . . . . . . . . . . . . 37

4.2.4 Sensitivity to the Normalization of the $\gamma$-ray Transmission Coefficient . . . . . . . . 38

4.2.5 Sensitivity to the Inclusion of Width Fluctuation Corrections . . . . . . . . . . . . . 39

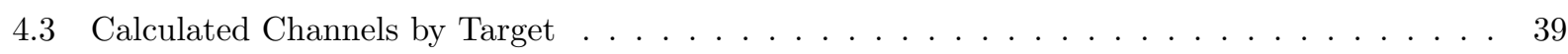

5 Conclusions $\quad 40$

A Cross Sections Included in the Detector Sets $\quad 45$

A.1 Cross Sections in the Existing RADCHEM Detector Sets . . . . . . . . . . . . . 45

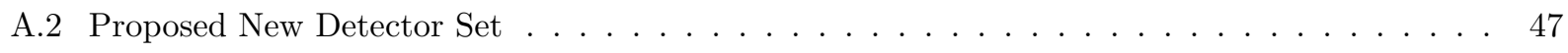

B Model Input Parameters $\quad \mathbf{5 4}$

B.1 Binding and Separation Energies $\ldots \ldots \ldots \ldots \ldots \ldots \ldots \ldots \ldots$

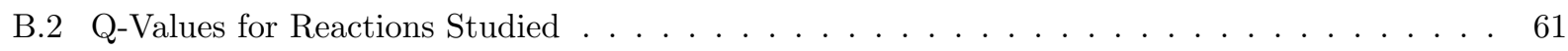

B.3 Modified Discrete Level Schemes . . . . . . . . . . . . . . . . . . . . . . 67

B.4 Level Density Parameters . . . . . . . . . . . . . . . . . . . . . 81

$\begin{array}{lr}\text { C Modeled Cross Sections Compared to Measurements } & \mathbf{8 8}\end{array}$

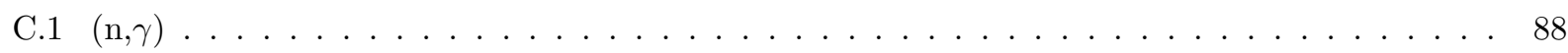

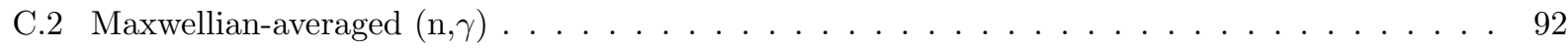

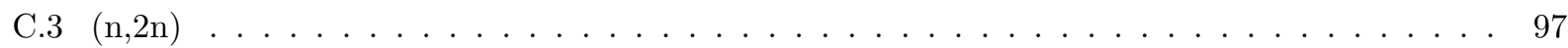

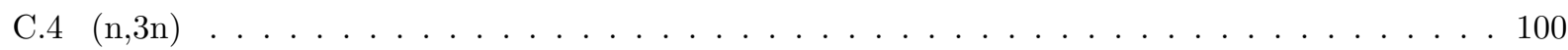

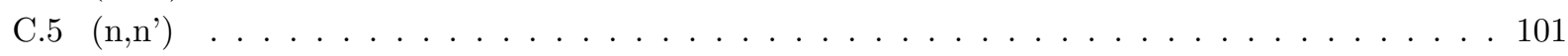

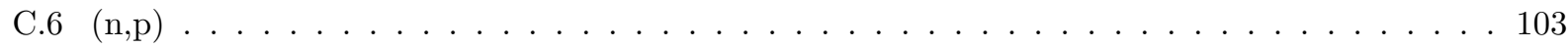

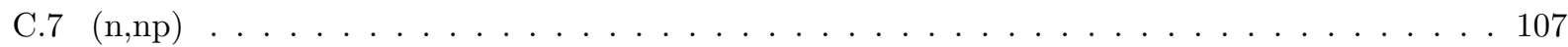

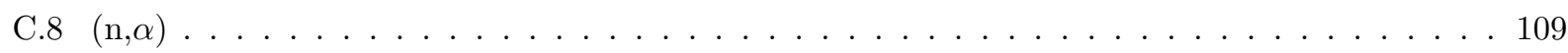

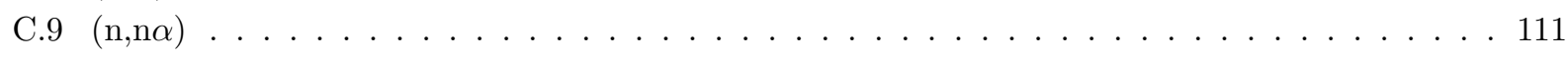

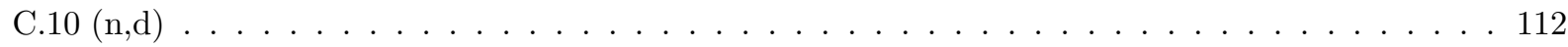

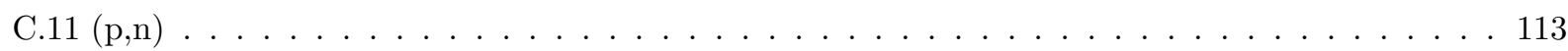

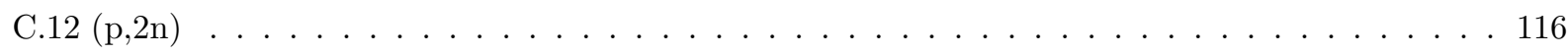

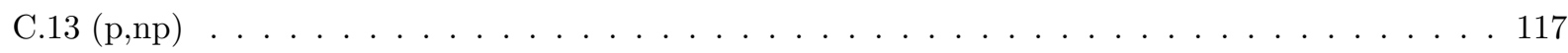

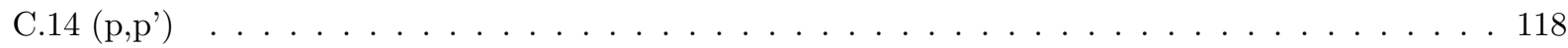

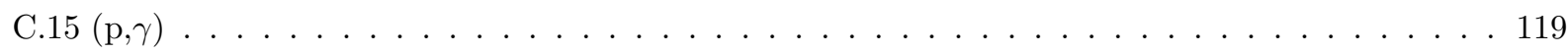

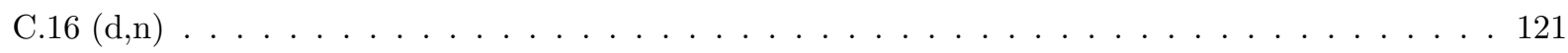

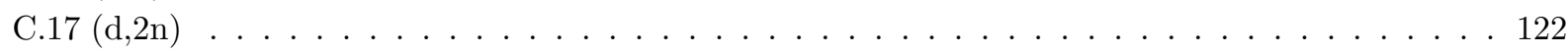

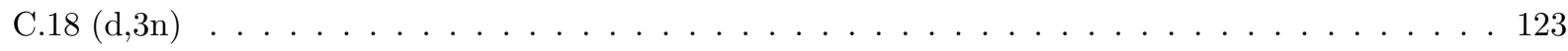

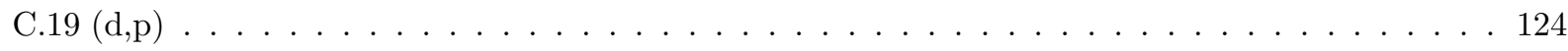

D Activation Cross Sections by Target $\quad 125$

\section{List of Figures}

1 Total measured neutron cross sections vs Koning-Delaroche for select targets _ . . . . . . . 12

2 Measured s- and p-wave strength functions and mean scattering radii vs Koning-Delaroche . 14 


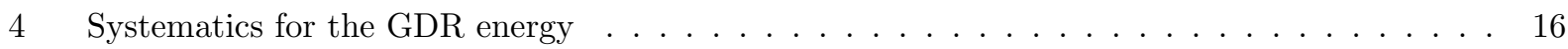

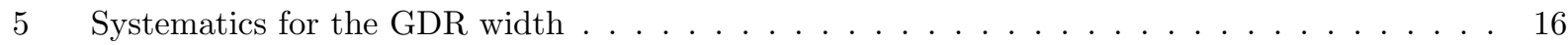

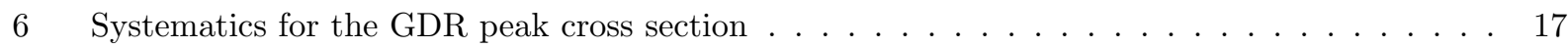

$7 \quad$ Systematics for average total s-wave radiation widths. $\ldots \ldots \ldots \ldots \ldots$

$8 \quad \chi^{2}$ fit to experimentally determined shell corrections, used to systematically determine unknown shell corrections. . . . . . . . . . . . . . . . . . . . . . . 19

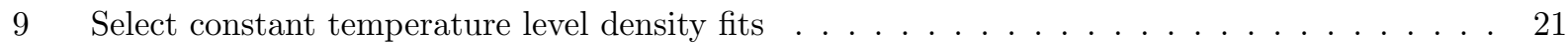

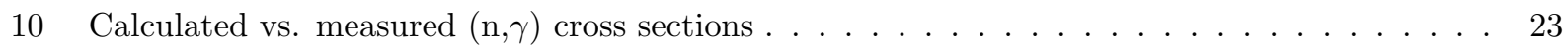

11 Calculated vs. recommended Maxwellian-averaged capture cross sections . . . . . . . . . . 25

12 Calculated vs. measured $(\mathrm{n}, 2 \mathrm{n})$ cross sections $\ldots \ldots \ldots \ldots \ldots \ldots \ldots \ldots$

13 Calculated vs. measured $(\mathrm{n}, \mathrm{p})$ cross sections $\ldots \ldots \ldots \ldots \ldots \ldots \ldots$

14 Calculated vs. measured $(\mathrm{n}, \mathrm{np})$ cross sections $\ldots \ldots \ldots \ldots \ldots \ldots$

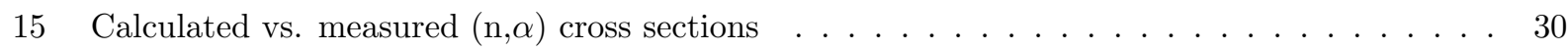

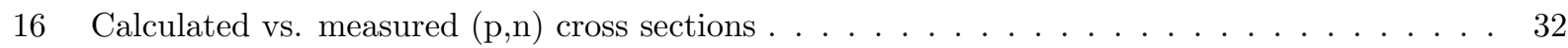

17 Select calculated $(\mathrm{n}, \mathrm{X})$ cross sections $\ldots \ldots \ldots \ldots \ldots \ldots \ldots \ldots \ldots$

18 Select calculated charged particle destruction cross sections . . . . . . . . . . . . . 34

19 Sensitivity of select activation cross sections to the $\langle F M\rangle$ parameter $\ldots \ldots \ldots \ldots$

20 Sensitivity of select activation cross sections to the alpha preformation parameter . . . . . . 37

21 Sensitivity to variations in the level density shell correction $\delta W \ldots \ldots \ldots \ldots$

22 Sensitivity to a $\pm 30 \%$ adjustment of the experimental s-wave average photon width $\Gamma_{\gamma} . \quad \ldots \quad 39$

23 Sensitivity to inclusion or exclusion of width fluctuation corrections $W \ldots \ldots \ldots$

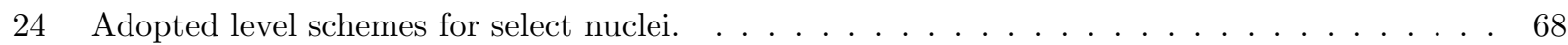

25 Modeled neutron capture cross sections compared to measurement . . . . . . . . . . . . . 88

26 Modeled Maxwellian-averaged neutron capture cross sections compared to measurement . . . 92

27 Modeled $(\mathrm{n}, 2 \mathrm{n})$ cross sections compared to measurement $\ldots \ldots \ldots \ldots \ldots \ldots$

28 Modeled $(\mathrm{n}, 3 \mathrm{n})$ cross sections compared to measurement $\ldots \ldots \ldots \ldots$. . . . . . . . 100

29 Modeled $\left(\mathrm{n}, \mathrm{n}^{\prime}\right)$ cross sections compared to measurement . . . . . . . . . . . . . . . . . 101

30 Modeled $(\mathrm{n}, \mathrm{p})$ cross sections compared to measurement . . . . . . . . . . . . . . . 103

31 Modeled $(\mathrm{n}, \mathrm{np})$ cross sections compared to measurement . . . . . . . . . . . . . . . 107

32 Modeled $(\mathrm{n}, \alpha)$ cross sections compared to measurement $\ldots \ldots \ldots \ldots$

33 Modeled $(\mathrm{n}, \mathrm{n} \alpha)$ cross sections compared to measurement . . . . . . . . . . . . . . . . . 111

34 Modeled $(\mathrm{n}, \mathrm{d})$ cross sections compared to measurement . . . . . . . . . . . . . . . 112

35 Modeled $(\mathrm{p}, \mathrm{n})$ cross sections compared to measurement $\ldots \ldots \ldots \ldots \ldots$

36 Modeled $(\mathrm{p}, 2 \mathrm{n})$ cross sections compared to measurement $\ldots \ldots \ldots \ldots \ldots$

37 Modeled $(\mathrm{p}, \mathrm{np})$ cross sections compared to measurement . . . . . . . . . . . . . . . . 117

38 Modeled $\left(\mathrm{p}, \mathrm{p}^{\prime}\right)$ cross sections compared to measurement $\ldots \ldots \ldots \ldots \ldots$

39 Modeled $(\mathrm{p}, \gamma)$ cross sections compared to measurement $\ldots \ldots \ldots \ldots$. . . . . . . . . 119

40 Modeled $(\mathrm{d}, \mathrm{n})$ cross sections compared to measurement $\ldots \ldots \ldots \ldots \ldots$. . . . . . . 121

41 Modeled $(\mathrm{d}, 2 \mathrm{n})$ cross sections compared to measurement . . . . . . . . . . . . . . . 122

42 Modeled $(\mathrm{d}, 3 \mathrm{n})$ cross sections compared to measurement . . . . . . . . . . . . . . 123

43 Modeled $(\mathrm{d}, \mathrm{p})$ cross sections compared to measurement . . . . . . . . . . . . . . . . . . 124

44 Activation cross sections for $\mathrm{N}=46$ targets of $\mathrm{Y}$, $\mathrm{Zr}$, and $\mathrm{Nb} \ldots \ldots \ldots \ldots$

45 Activation cross sections for $\mathrm{N}=47$ targets of $\mathrm{Y}, \mathrm{Zr}$, and $\mathrm{Nb} \ldots \ldots \ldots \ldots$

46 Activation cross sections for $\mathrm{N}=48$ targets of $\mathrm{Y}, \mathrm{Zr}, \mathrm{Nb}$, and $\mathrm{Mo} \ldots \ldots \ldots$

47 Activation cross sections for $\mathrm{N}=49$ targets of $\mathrm{Y}, \mathrm{Zr}, \mathrm{Nb}$, and $\mathrm{Mo} \ldots \ldots \ldots$ 
48 Activation cross sections for $\mathrm{N}=50$ targets of $\mathrm{Y}, \mathrm{Zr}, \mathrm{Nb}$, and $\mathrm{Mo} \ldots \ldots \ldots$

49 Activation cross sections for $\mathrm{N}=51$ targets of $\mathrm{Y}, \mathrm{Zr}, \mathrm{Nb}$, and $\mathrm{Mo} \ldots \ldots \ldots$

50 Activation cross sections for $\mathrm{N}=52$ targets of $\mathrm{Y}, \mathrm{Zr}, \mathrm{Nb}$, and $\mathrm{Mo} \ldots \ldots \ldots$

51 Activation cross sections for $\mathrm{N}=53$ targets of $\mathrm{Y}, \mathrm{Zr}, \mathrm{Nb}$, and $\mathrm{Mo} \ldots \ldots \ldots$

52 Activation cross sections for $\mathrm{N}=54$ targets of $\mathrm{Y}, \mathrm{Zr}, \mathrm{Nb}$, and $\mathrm{Mo} \ldots \ldots \ldots$

53 Activation cross sections for $\mathrm{N}=55$ targets of $\mathrm{Zr}, \mathrm{Nb}$, and $\mathrm{Mo} \ldots \ldots \ldots \ldots$

54 Activation cross sections for $\mathrm{N}=56$ targets of $\mathrm{Zr}, \mathrm{Nb}$, and $\mathrm{Mo} \ldots \ldots \ldots \ldots$

\section{List of Tables}

1 Comparison of our modeled $(\mathrm{n}, \gamma)$ cross sections to experimental data at $30 \pm 2 \mathrm{keV} \ldots \ldots$

2 Comparison of our modeled Maxwellian-averaged $(\mathrm{n}, \gamma)$ cross sections (in millibarns) to recommended values at $30 \mathrm{keV} \ldots \ldots \ldots \ldots \ldots \ldots \ldots$

3 Comparison of our modeled $(\mathrm{n}, 2 \mathrm{n})$ cross sections to experimental data at $14.1 \pm 0.1 \mathrm{MeV}$. . . 28

4 Sensitivity of critical $14.1 \mathrm{MeV}(\mathrm{n}, 2 \mathrm{n})$ cross sections to the $\langle F M\rangle$ parameter $\ldots \ldots \ldots . \ldots 35$

5 Cross sections available in existing RADCHEM detector sets . . . . . . . . . . . . . . 45

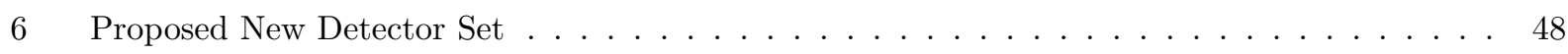

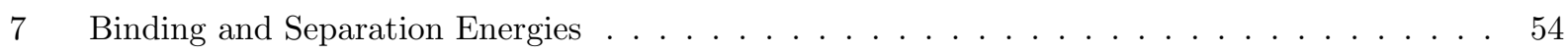

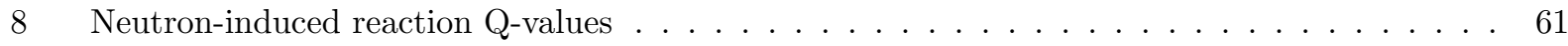

9 Charged-particle reaction $\mathrm{Q}$-values $\ldots \ldots \ldots \ldots \ldots \ldots \ldots$

$10 \quad$ Level Density Parameters . . . . . . . . . . . . . . . . . . . . . . 81 


\subsection{Current Detector Sets}

Over the last 40 years a number of detector sets have been developed at LLNL and LANL. Twenty-three neutron threshold detector sets and five charged particle sets are currently available. The sets of interest in this modeling effort are as follows:

- Yttrium neutron-induced set (YT0585), used to calculate the production of ${ }^{87} \mathrm{Y}$ $\left(t_{1 / 2}=3.317 \mathrm{~d}\right),{ }^{87 m} \mathrm{Y}\left(t_{1 / 2}=13.37 \mathrm{~h}\right)$, and ${ }^{88} \mathrm{Y}\left(t_{1 / 2}=106.7 \mathrm{~d}\right)$ from stable ${ }^{89} \mathrm{Y}$.

- Yttrium charged-particle set (YT0488), used in conjunction with sets YT0585 and Zr0982 to calculate the production of zirconium isotopes from stable ${ }^{89} \mathrm{Y}$.

- Zirconium neutron-induced set (Zr0982), used to calculate the production of ${ }^{87} \mathrm{Zr}$ $\left(t_{1 / 2}=1.68 \mathrm{~h}\right),{ }^{88} \mathrm{Zr}\left(t_{1 / 2}=82.6 \mathrm{~d}\right)$, and ${ }^{89}{ }^{8 \mathrm{Zr}}\left(t_{1 / 2}=3.268 \mathrm{~d}\right)$ from stable zirconium.

- Niobium neutron-induced set (Nb0179), used to calculate the production of ${ }^{92 m} \mathrm{Nb}$ $\left(t_{1 / 2}=10.15 \mathrm{~d}\right)$ from stable ${ }^{93} \mathrm{Nb}$.

- Molybdenum neutron-induced set (Mo1278), used to calculate the production of ${ }^{88} \mathrm{Zr}$ $\left(t_{1 / 2}=82.6 \mathrm{~d}\right),{ }^{89} \mathrm{Zr}\left(t_{1 / 2}=3.268 \mathrm{~d}\right)$, and ${ }^{93 m} \mathrm{Nb}\left(t_{1 / 2}=16.13 \mathrm{y}\right)$ from stable molybdenum.

The cross sections available in these detector sets, as listed in (Nethaway 1998) are summarized in table 5 in appendix A.1. Most of the cross sections are taken from calculations performed at LLNL and LANL between 1972 and 1988. The chargedparticle reactions on ${ }^{89 g} \mathrm{Y}$ are taken from measurement. Several other cross sections (primarily $(\mathrm{n}, 2 \mathrm{n})$ reactions on stable targets) have been scaled to match measured cross section data at or around $14.1 \mathrm{MeV}$ of incident energy. These sets can be accessed on the world wide web at http://nuclear.llnl.gov/CNP/nads/main.html.

\subsection{Motivation for Updating the Detector Sets}

Previous successes in updating other detector sets suggest that the YT0488, YT0585, Zr0982, Nb0179, and Mo1278 sets may also benefit from a new evaluation.

Many of the RADCHEM detector sets updated in our previous modeling efforts had a clear need for improvement Some of the more disturbing shortfalls of these sets included neutron capture cross sections being copied from other targets and adjusted, $(\mathrm{n}, 2 \mathrm{n})$ cross sections with an assumed maximum cross section and standard shape rising from a calculated threshold, and educated guesses for extrapolating cross sections to higher or lower energies. In each of these cases it is preferable to model the cross section using all available input data and then scale the cross section to match a measured data point if necessary (i.e. scale a cross section with a calculated shape).

The detector sets considered in the present work do not suffer from such deficiencies. Every cross section included was either measured or calculated. However, we sometimes find that only a few neutron-induced reactions of interest for a given target were calculated explicitly. The remaining reaction channels (in $\mathrm{Y}$ ) were lumped together as a so-called $(\mathrm{n}, \mathrm{X})$ "destruction" cross section. In several instances, the $(\mathrm{n}, \mathrm{X})$ cross section is significantly larger than the channels explicitly calculated. In these cases, it is desirable to know which reactions dominate at a given incident energy. Furthermore those reactions should be explicitly included in the network so that the largest nuclear flows can be properly accounted for. The detector sets of interest in this work also have several instances where a dominant reaction channel (neutron capture for ${ }^{92} \mathrm{Nb}$, for example) was grouped in with an $(\mathrm{n}, \mathrm{X})$ destruction cross section.

Another motivation is drawn from the general improvement in cross section modeling capabilities. In the nearly two decades since these sets were developed, many new cross section measurements have been performed, and the amount of nuclear structure data used to constrain model parameters has increased. Additionally, several efforts have been made to develop consistent approaches to modeling nuclear reaction cross sections (Belgya et al. 2005), and there are more accurate methods of calculating and estimating cross sections for which we have no data.

Thirdly, our proposed new evaluation of these cross sections will include an in-depth investigation into the sensitivity of the modeled cross sections to variations in the various statistical model inputs. In doing so we are able to determine which parameters are the most important for a given reaction. This also allows us to estimate how much a calculated cross section will change if new experimental measurements place more constraints on 
the model inputs.

\subsection{Proposed Detector Sets}

We consider as targets each of the isotopes listed in Table 6 of Appendix A.2. For each of these targets, we model the reaction channels indicated in the table. In cases where the residual nucleus has a long-lived isomer (which we define as $t_{1 / 2}>1 \mu \mathrm{s}$ ), we model individual cross sections leading to the ground and isomeric states, as well as an "activation" cross section, defined as the total cross section producing a given isotope. This modeling effort includes all of the reactions previously available in the RADCHEM detector sets, but also includes many additional targets and reaction channels. These additional reactions are included primarily to provide further comparisons to measured cross section data and bolster our confidence in the accuracy of cross sections modeled for unstable targets. These reactions also account for the various possible destruction reactions that are significant in this mass range.

Our goal is to develop a consistent set that reproduces, as closely as possible, measured cross sections on targets in the local region of interest. To do this we develop local systematics for the many input quantities used in the theoretical reaction modeling calculations. These systematics are based on experimental data that are often only available for compound nuclear systems formed from a stable target plus a neutron. Of course, we use experimental data whenever it is available, but reactions proceeding through unstable systems are unavoidable in radiochemistry. Short of developing new experimental techniques to measure cross sections on unstable targets, our only hope of reproducing measured activity from UGT shots, and addressing the uncertainty associated with the nuclear cross sections, is to develop cross section sets that reproduce well the measured cross sections in the local region of interest.

In $\S 2$ we describe the theoretical techniques used in the modeling effort. $\S 3$ describes the input parameters. $\S 4$ gives results. We conclude with $\S 5$.

\section{Nuclear Reaction Theory}

\subsection{Reaction Mechanisms}

Conceptually, we consider nuclear reaction mechanisms to be of two general types: direct processes and compound processes. Direct processes can be pictured as simple interactions of the incident particle with the nuclear potential of the target nucleus. They proceed on a rapid time scale (of order $\sim 10^{-22} \mathrm{~s}$ ), and the reaction products are often highly peaked in the incident particle direction. Direct reactions are generally quite small over the energy range of interest in this study, and have not been included in our calculations.

Compound processes are pictured as complicated interactions proceeding over a much longer timescale $\left(10^{-15}-10^{-18} \mathrm{~s}\right)$ in which the reaction is mediated by the formation of a "compound nucleus", with the excitation energy of the incident particle being statistically "shared" with the ensemble of nucleons in the target over all energetically allowed degrees of freedom. The reaction products are largely isotropic.

Other intermediate reaction mechanisms exist between these two extremes. We refer to these as "pre-equilibrium" nuclear processes, where a particle may be emitted from the target+projectile compound system prior to equilibration. Over the energy range of interest to this project (a few $\mathrm{keV}$ to $20 \mathrm{MeV}$ ) we will consider pre-equilibrium and compound nuclear processes, with the preequilibrium processes operating principally above $10 \mathrm{MeV}$ of incident particle energy.

\subsection{Hauser-Feshbach Statistical Model}

A traditional theoretical approach to compound nuclear reactions is the statistical or HauserFeshbach model (Hauser \& Feshbach 1952). This model is valid for high level densities in the compound nucleus, allowing one to use energy averaged transmission coefficients $T$, which describe absorption via an imaginary part in the (optical) nucleon-nucleus potential (Mahaux \& Weidenmüller 1979). For the reaction I (in state $\mu$ ) $+j \rightarrow k+\mathrm{L}$ (in state $\nu$ ), with $\mathrm{I}^{\nu}+j$ interacting with center-of-mass energy $\mathrm{E}_{j}^{\mu}$ (in $\mathrm{MeV}$ ), the average cross section is given by

$$
\sigma_{j k}^{\mu \nu}\left(E_{j}^{\mu}\right)=\frac{\pi \lambda_{j}^{2}}{g_{I}^{\mu} g_{j}} \sum_{J, \pi} g_{J} \frac{T_{j}^{\mu}\left(J^{\pi}\right) T_{k}^{\nu}\left(J^{\pi}\right)}{T_{t o t}\left(J^{\pi}\right)} W\left(J^{\pi}\right)
$$

where the summation extends over all compound nuclear spins and parities $J^{\pi}, \mu$ and $\nu$ are states in the target and product $(=0$ for the ground state, 1 for the $1^{\text {st }}$ excited state, etc.). The cross section has units of area, described by $\pi \lambda_{j}^{2}=$ $0.6566\left(\hat{A}_{j} E_{j}^{\mu}\right)^{-1}$ barns, with $\hat{A}_{j}=\left(A_{I} A_{j}\right) /\left(A_{I}+\right.$ $\left.A_{j}\right)$ being the reduced mass in atomic mass units and $E_{j}^{\mu}$ is the center of mass energy in units of 
$\mathrm{MeV} . \lambda_{j}$ is the wavelength related to the wave number $k_{j}$ in the target plus incident particle channel by $\lambda_{j}=1 / k_{j}$ The statistical weights are given by $g_{y}^{x}=\left(2 J_{y}^{x}+1\right)$. Items without superscripts refer to the compound nucleus.

The transmission coefficients in the numerator are given by $T_{j}^{\mu}\left(J^{\pi}\right)=$ the total transmission coefficient for forming the state $J^{\pi}$ in the compound nucleus $I^{\mu}+j$ at energy $E_{j}^{\mu}$. Likewise, $T_{k}^{\nu}\left(J^{\pi}\right)$ is the same as $T_{j}^{\mu}\left(J^{\pi}\right)$ but for the pair $L^{\nu}+k$ at energy $E_{k}^{\nu}$. Implicit in these definitions is a sum over all possible $l$-waves and channel spins, i.e.

$$
T_{j}^{\mu}\left(J^{\pi}\right)=\sum_{l, s} T_{j}^{\mu}\left(J^{\pi}, l, s\right)
$$

where $l$ is any partial wave number (orbital angular momentum) that can couple the state $\mu$ to the compound nuclear state having spin and parity $J^{\pi}$ subject to quantum mechanical selection rules and $s$ is the vector sum of the spins $J_{I}^{\mu}$ and $J_{j}$. Hence $s$ takes on all integer (or half-integer) numbers from $\left|J_{I}^{\mu}-J_{j}\right|$ to $J_{I}^{\mu}+J_{j}$.

$T_{\text {tot }}$ represents the sum of transmission coefficients over all possible decay channels (i.e. for all particles and photons). The cross section for the formation of species L, regardless of its state $\nu$, is obtained by summing Eq. [1] over all bound states $\nu$ of $\mathrm{L}$ for which the reaction is energetically allowed.

When evaluating these sums, if energies become of interest which exceed the highest discrete excited state for which energy, spin, and parity are explicitly known, a nuclear level density formula must be employed. Specifically, the definitions for the transmission coefficients $T_{j}\left(J^{\pi}\right), T_{k}\left(J^{\pi}\right)$, and $T_{t o t}\left(J^{\pi}\right)$ must be modified:

$$
\begin{aligned}
& T_{k}\left(J^{\pi}\right)=\sum_{\nu=0}^{\omega} T_{k}^{\nu}\left(J^{\pi}\right)+ \\
& \sum_{J^{\nu} \pi^{\nu}} \int_{\xi_{L}^{\omega}}^{\xi_{L}^{\text {max }}} T_{k}^{\nu}\left(\xi_{L}^{\nu}, J^{\pi}\right) \rho\left(\xi_{L}^{\nu}, J^{\nu}, \pi^{\nu}\right) d \xi_{L}^{\nu} d \pi^{\nu} d J^{\nu}
\end{aligned}
$$

where for the nucleus $\mathrm{L}, \xi_{L}^{\omega}$ is the energy of the highest excited state, $\omega$, of known energy, spin, and parity; $\xi_{L}^{\max }=E_{k}^{0}=E_{j}^{0}+Q_{j k}$ is the maximum excitation energy available, and $\rho\left(\xi_{L}^{\nu}, J^{\nu}, \pi^{\nu}\right)$ is the density of states per unit energy of spin and parity $J^{\nu}$ and $\pi^{\nu}$ at the excitation energy $\xi_{L}^{\nu}$. The above integral approximates a sum and is subject to the same quantum mechanical restrictions implied in the definition of the transmission function.

\subsection{Width Fluctuations}

In addition to the ingredients required for Eq. [1], we apply width fluctuation corrections $\left(W\left(J^{\pi}\right)\right.$, hereafter WFC), which define correlation factors with which all partial channels of incoming particle $j$ and outgoing particle $k$, passing through excited state $(E, J, \pi)$, should be multiplied. The major effect is to enhance the elastic channel and accordingly decrease the other open channels. They are most often observed at or near channel opening energies, for example when a $(\mathrm{p}, \gamma)$ and a $(\mathrm{p}, \mathrm{n})$ channel compete and the weaker $(\mathrm{p}, \gamma)$ channel is enhanced. Above a few $\mathrm{MeV}$ of excitation energy, when many competing channels are open, WFC's can be neglected.

A reasonably complete treatment for the WFC, obtained with the Gaussian orthogonal ensemble (GOE) approach, requires the evaluation of a triple integral and to date has been considered much to costly to apply in nuclear cross section calculations. Several approximations have been developed, the most popular ones are the Moldauer model (Moldauer 1976), and the HRTW model (Hofmann et al. 1975). We use the Moldauer model approximation in this study. For a detailed description of the full (GOE) treatment and a comparison with the Moldauer and HRTW approximation models mentioned above, see (Hilaire Lagrange \& Koning 2003).

\subsection{Pre-Equilibrium Processes}

For excitation energies starting around 10 $\mathrm{MeV}$, pre-equilibrium processes become important. The pre-equilibrium cross section is subtracted from the total reaction cross section leading to the first compound nucleus, and is usually unimportant for subsequent compound nuclei. Here we describe equilibration of the compound nuclear system in terms of the exciton model (Cline \& Blann 1971) including alpha particle emission (Milazzo-Colli \& Braga-Marcazzan 1973). We adopt an initial 2-particle 1-hole configuration. Average rates for internal transitions, corrected for the Pauli principle by Cline (1972), are related by the formulas of Williams (1970) to the absolute square of the average effective matrix element $|M|$ of the residual interactions as per Eq. [7] of (Uhl \& Strohmaier 1976). The dependence of $|M|^{2}$ on mass number and excitation energy is

$$
|M|^{2}=\langle F M\rangle A^{-3} E^{-1}
$$

The description of alpha particle emission in the 
pre-equilibrium model is a straightforward extension of nucleon emission, assuming nucleons preform alpha clusters. In making such an extension, one introduces a parameter $\phi$ which represents the probability that the incoming particle will strike a pre-formed alpha cluster.

In the pre-equilibrium stage of the reaction, particle emission is assumed to be the only decay mode. For the equilibration, the WFC corrected Hauser Feshbach formula (Eq. [1]) is applied. All subsequent processes are treated as sequential evaporation steps.

\subsection{The STAPRE Hauser-Feshbach Reac- tion Code}

We model our cross sections using the statistical model code STAPRE (Uhl \& Strohmaier 1976), which embodies all of the physical models discussed above. The version of the code we use is STAPRE-H95 (Avrigeanu \& Avrigeanu 1976), available from the NEA web site. We have made several modifications, primarily to the level density routines. Prior versions of the code were used to develop parts of the existing RADCHEM data sets (Vonach 1982).

In the following we discuss the important ingredients of statistical model calculations, and the methods utilized to estimate them. These are the requisite nuclear structure data, such as the binding energies of all nuclei included (which define the separation and reaction threshold energies and Q-values of the various reaction channels considered), as well as the energies, spins, and parities of the ground states and all known excited states of these nuclei, and the detailed branching ratios for the gamma-ray cascade from excited to lowlying states. Also needed are parameters controlling the width fluctuation corrections and the preequilibrium model, the particle and $\gamma$-transmission coefficients, and the nuclear level densities of all nuclei involved in a given reaction. The reliability with which these ingredients can be calculated determines the accuracy (or reliability) of a given cross section calculation.

\subsection{Breakup of Incident Deuterons}

Due to its relatively weak binding, an incident deuteron may be separated into its constituent nucleons in the presence of the Coulomb barrier. The now separate nucleons are then free to interact with the target nucleus by either scattering (elastically or inelastically) or fusing to the target. The STAPRE code does not address these so-called "deuteron breakup" processes. We must first calculate the breakup and breakup-fusion cross sections using the methods of (Udagawa \& Tamura 1986) and express the resulting total breakup/breakup-fusion cross section as a fraction of the reaction cross section. The reaction cross section in the STAPRE code can then be reduced by the appropriate amount.

A more thorough treatment of deuteron breakup would allow the excited target (in the case of an inelastically scattered nucleon) or compound nucleus (in the case of breakup-fusion) to decay via the usual statistical models. However, this capability has not yet been incorporated into our suite of reaction codes.

\subsection{Determination of Destruction Reac- tions}

Reaction channels other than those listed explicitly in Appendix A.2 may comprise a significant portion of the total reaction cross section. Rather than attempt to calculate all kinematically allowed channels explicitly, we supply a "destruction" cross section to account for additional depletion of a given target. The destruction cross section is determined by subtracting from the reaction cross section the sum of the compound elastic and all other explicitly calculated channels. The remaining cross section is designated $(n, X),(p, X)$, or $(\mathrm{d}, \mathrm{X})$ depending on the incident particle. Since we have explicitly calculated most of the important neutron induced channels, our $(\mathrm{n}, \mathrm{X})$ cross sections are expected to be quite small.

The greatest obstacle faced in calculating destruction cross sections is the numerical precision at which cross sections are stored in the computer memory or files. To eliminate numerical "noise" that arises from these precision issues, we apply a sequence of filters to our "raw" calculated destruction cross sections. First, since we have included all open channels up to about $3 \mathrm{MeV}$ of incident energy for each incident particle considered in this study, we set any resulting destruction cross section below $3 \mathrm{MeV}$ to zero (actually to 1 nanobarn, which is in practice equal to zero for network calculations). Any point in the energy grid where the cross section is non-zero but the cross section at the grid point to both sides is zero (i.e. "spikes" that show up in the cross section) are also set to zero. A two step Savitsky-Golay smoothing filter (linear over a short range and quadratic over a larger range) is then applied. Any cross section lower than 1 millibarn or $0.1 \%$ of the reaction 
cross section (whichever is greater) is then set to zero, and the smoothing filter applied again. This is followed by one last search for "spikes". A comparison of the raw destruction cross section and the filtered one indicates that the filtering process does not significantly alter the "real" destruction cross section (i.e. the destruction cross section due to unaccounted reaction channels, as opposed to that arising from precision error).

This concludes our discussion of the methods and models used in our calculations. we now proceed to describe the various quantities used as input in these models.

\section{Inputs for the Reaction Models}

\subsection{Nuclear Structure Data}

\subsubsection{Ground State Masses and $J^{\pi}$ Assignments}

We adopt for nuclear masses the experimental mass excess values of (Wapstra et al. 2003; Audi et al. 2003). In the event that an experimental mass excess is not available, we adopt the values from the finite range droplet model (FRDM) (Möller et al. 1995). Spin and parity assignments are from (Tuli 2000). In appendix B.1 we present the binding energies (in $\mathrm{MeV}$, calculated from the adopted masses) and the separation energies for neutrons, protons, $\alpha$-particles, and deuterons for each of the nuclei included in this study. Ground state spin and parity assignments are given in Table 6 (Appendix A.2). In Appendix B.2, we provide reaction $\mathrm{Q}$-values for the cross sections modeled in this study.

\subsubsection{Nuclear Level Schemes}

The nuclear structure data needed to model the gamma-ray cascade was adopted from (Belgya et al. 2005). We include all levels up to the energy where spins, parities, and $\gamma$-ray branching ratios have been unambiguously assigned. For several isotopes, including ${ }^{76-83} \mathrm{Br}$, ${ }^{77-84} \mathrm{Kr},{ }^{78} \mathrm{Rb},{ }^{83-89} \mathrm{Sr},{ }^{84-91} \mathrm{Y}$, and ${ }^{86-91} \mathrm{Zr}$, additional evaluations were performed by R. Bauer (Bauer 2003). The modified nuclear level schemes for the bromine, krypton, and rubidium isotopes can be found in (Hoffman et al. 2004a). The remaining modified schemes, including level energies, spin and parity assignments, and $\gamma$-ray branching ratios, may be found in appendix B.3.

Occasionally we encounter a situation where, due to missing spin/parity assignments or branching ratios, an isomer is not included in the level scheme. In most of these cases, the only missing data was branching ratios. In order to include the isomers in our calculations, we have estimated the missing branching ratios as follows:

- ${ }^{94} \mathrm{Y}$ : First excited state decays to the ground state (only possible $\gamma$-ray decay).

- ${ }^{90} \mathrm{Zr}$ : Branching ratios were removed from the first and second excited states $(0+$ at $1.761 \mathrm{MeV}, 2+$ at $2.186 \mathrm{MeV})$. Even though these states have very short lifetimes (61.3 ns and 88.4 fs, respectively), they were treated as isomers in the RADCHEM Zr0982 detector set. We have also considered these states as isomers to maintain consistency with the RADCHEM set.

- ${ }^{91} \mathrm{Nb}:$ In order to include the $17 / 2$ - isomer at $2.034 \mathrm{MeV}$, we assume that the second excited state $(7 / 2$ - at $1.040 \mathrm{MeV})$ decays by an E1 transition to the $9 / 2+$ ground state. We also assume that the $9 / 2+$ level at 1.885 $\mathrm{MeV}$ decays $67 \%$ of the time by E1 to the $7 / 2$ - state at $1.040 \mathrm{MeV}$ and $33 \%$ of the time by M1 to the $7 / 2+$ state at $1.581 \mathrm{MeV}$. Since the first isomer lies below these states $(1 / 2$ at $0.104 \mathrm{MeV}$ ), these assumed branching ratios will have an effect on our modeled cross sections, particularly those that have ${ }^{91} \mathrm{Nb}$ as a residual.

- ${ }^{90}$ Mo: Filled in several branching ratios for levels between the $0+$ ground state and the 8- isomer at $2.875 \mathrm{MeV}$. Since any population of these levels will eventually decay to the ground state, the choice of branching ratios will not affect our modeled cross sections.

- ${ }^{93}$ Tc: For the $7 / 2$ - level at $1.555 \mathrm{MeV}$, we assume an E1 transition to the ground state. This branching ratio was needed in order to include the $17 / 2$ - isomer at $2.185 \mathrm{MeV}$. This choice of branching could affect the amount of cross section going to the ground state and first isomer at $0.392 \mathrm{MeV}$.

- ${ }^{98}$ Tc: Assumed that the third excited state (3- at $0.073 \mathrm{MeV}$ ) decays via E1 to the second excited state. This is done in order to include the 2 - isomer at $0.091 \mathrm{MeV}$ in our level scheme. There are no isomers between this state and the ground state, so this assumed branching ratio will not affect our modeled cross sections. 
Each of these modified level schemes are also included in appendix B.3. For ${ }^{100} \mathrm{Rh}$ the number of missing levels, spins, and/or parities prevented the inclusion of the isomer in our calculations.

A few of the nuclei included in this study have a first excited state that is nearly degenerate in energy with the ground state. Since having two levels with the same energy cannot be handled by our Hauser-Feshbach code, we have increased the energy of the first excited state for these nuclei to $10 \mathrm{eV}$. The isotopes affected are ${ }^{96} \mathrm{Y},{ }^{98} \mathrm{Y},{ }^{87} \mathrm{Nb}$, ${ }^{88} \mathrm{Nb},{ }^{89} \mathrm{Nb},{ }^{91} \mathrm{Ru},{ }^{94} \mathrm{Rh}$, and ${ }^{98} \mathrm{Rh}$.

In some cases a visual inspection of the cumulative number of levels as a function of energy suggests that levels are missing from the discrete level scheme. In such cases, we reduce the number of discrete levels used in our calculations (see section 3.3.3). In cases where no discrete level data is available we only include a ground state in our calculations.

\subsection{Transmission Coefficients}

Energy-averaged transmission coefficients are needed for each particle considered as a possible exit channel in the Hauser-Feshbach denominator. In our modeling effort, we include outgoing neutrons, protons, $\alpha$-particles, deuterons, and photons. We do not include other light particles (such as tritons and ${ }^{3} \mathrm{He}$ ).

\subsubsection{The Neutron and Proton Optical Potential}

For the calculation of the neutron and proton particle transmission coefficients, we adopt the optical model of (Koning \& Delaroche 2003). Although they have tuned their parameters to fit data for many different species (see their Tables 6 and 7), we use the global nucleon-nucleon optical model potential (OMP), as it gives a very satisfactory fit to measured total neutron cross section data and measured total proton reaction cross section data in the range of interest to us. Specifically, we adopt the potential depth parameters and Fermi energies for the neutron and proton global OMP defined in their Section 5.2, tables 14 and 15. The particle transmission coefficients were generated by the optical model code ECIS96 (Raynal 1996). Although designed for coupled channel calculations, we used the code in a spherical optical model mode.

We present in Figure (1) results of the Koning \& Delaroche optical model compared to measured total neutron cross sections for select targets in our region of interest. For nuclei with $A<96$, the optical model prediction (solid black line) closely replicates the measured total neutron cross sections above $\sim 500 \mathrm{keV}$. At lower energies, the measured cross sections generally exhibit structure due to individual resonances. This structure cannot be reproduced by an optical model, which only predicts average cross sections.

We have performed additional comparisons for total neutron cross sections on targets of strontium, technetium, and rhodium, with similar results. This optical potential has also produced favorable comparisons to measured total neutron cross sections in other regions of the periodic chart (Hoffman et al. 2004a; Hoffman et al. 2004b; Hoffman et al. 2004c; Kelley et al. 2005; Kelley et al. 2006). Further evaluations are provided in (Koning \& Delaroche 2003).

For nuclei with $A \geq 96$, the optical model over predicts the measured total neutron cross section by roughly $50 \%$ between $\sim 80-500 \mathrm{keV}$. One possible cause is that we have neglected nuclear deformations. The heavier nuclei in our region of interest are somewhat deformed, and the collectivity of the even-even nuclei (defined as the ratio of the energies of the first $J^{\pi}=4^{+}$to $J^{\pi}=2^{+}$ levels) above $A=96$ have values ranging between 2.0 and 2.6, indicating that the nuclei may be better treated as deformed vibrators. However, the loaded detector elements considered in this study (stable yttrium, zirconium, niobium, and molybdenum) almost all lie below $A=96$. All of the reactions that will significantly affect the production of the radioactive species of interest lie at or below $A=92$. Thus, we may adopt the simpler spherical model without introducing significant errors into the modeled cross sections.

Further information regarding the quality of the neutron optical potential may be obtained by comparing the s- and p-wave strength functions and mean scattering radii predicted by the model to measured values. We make such a comparison in Figure 2. Each of these plots show the ratio of the quantity predicted by the optical potential to the measured value, plotted against the mass of the compound (target plus neutron) system. The error bars reflect this same ratio using the upper and lower errors in the measured values. Hence, if the error bars cross unity, the optical model prediction is within the errors of the measured value. For the s-wave strength functions $\left(S_{0}\right)$, we see that many of the optical model predictions lie within the errors of the measured values. In all cases, the modeled/measured ratio is with a factor of two (in- 

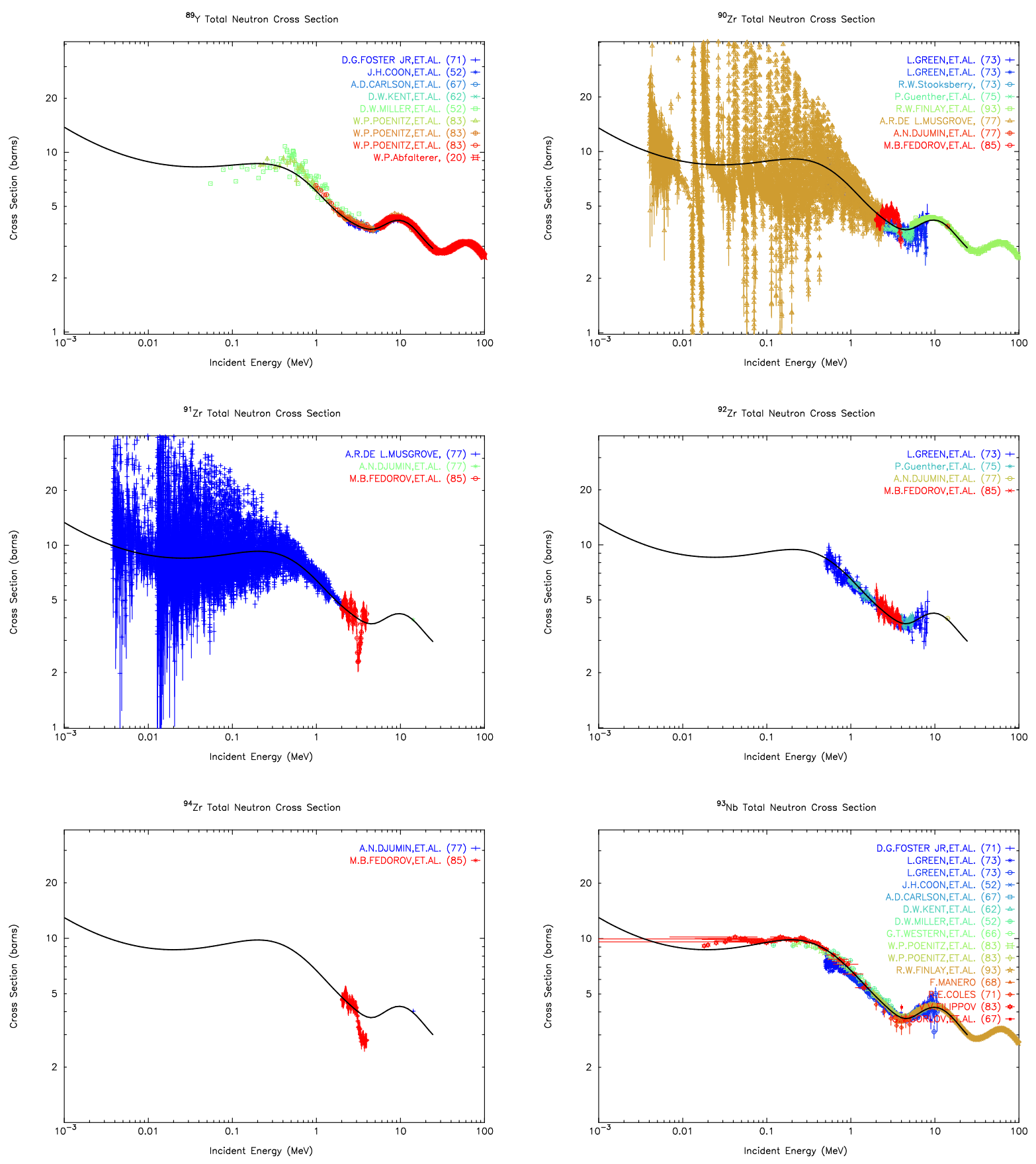

Fig. 1.- Total measured neutron cross sections vs those predicted by the optical model of Koning-Delaroche for select targets in the range $38 \leq Z \leq 45$. Measured values were obtained from (EXFOR 2006). The optical model prediction is indicated by the solid black line.

dicated by the two outer dotted lines). Similarly, the predicted p-wave strength functions $\left(S_{1}\right)$, also fall within a factor of two of the measured values. The mean scattering radii predicted by the optical potential $\left(R^{\prime}\right)$, are slightly higher than the measurements.
There is some evidence that the proton transmission coefficients derived from this optical potential may be too large for lighter nuclei (Kelley et al. 2005). To gauge whether or not the proton transmission function is also too large for nuclei in the current region of interest 

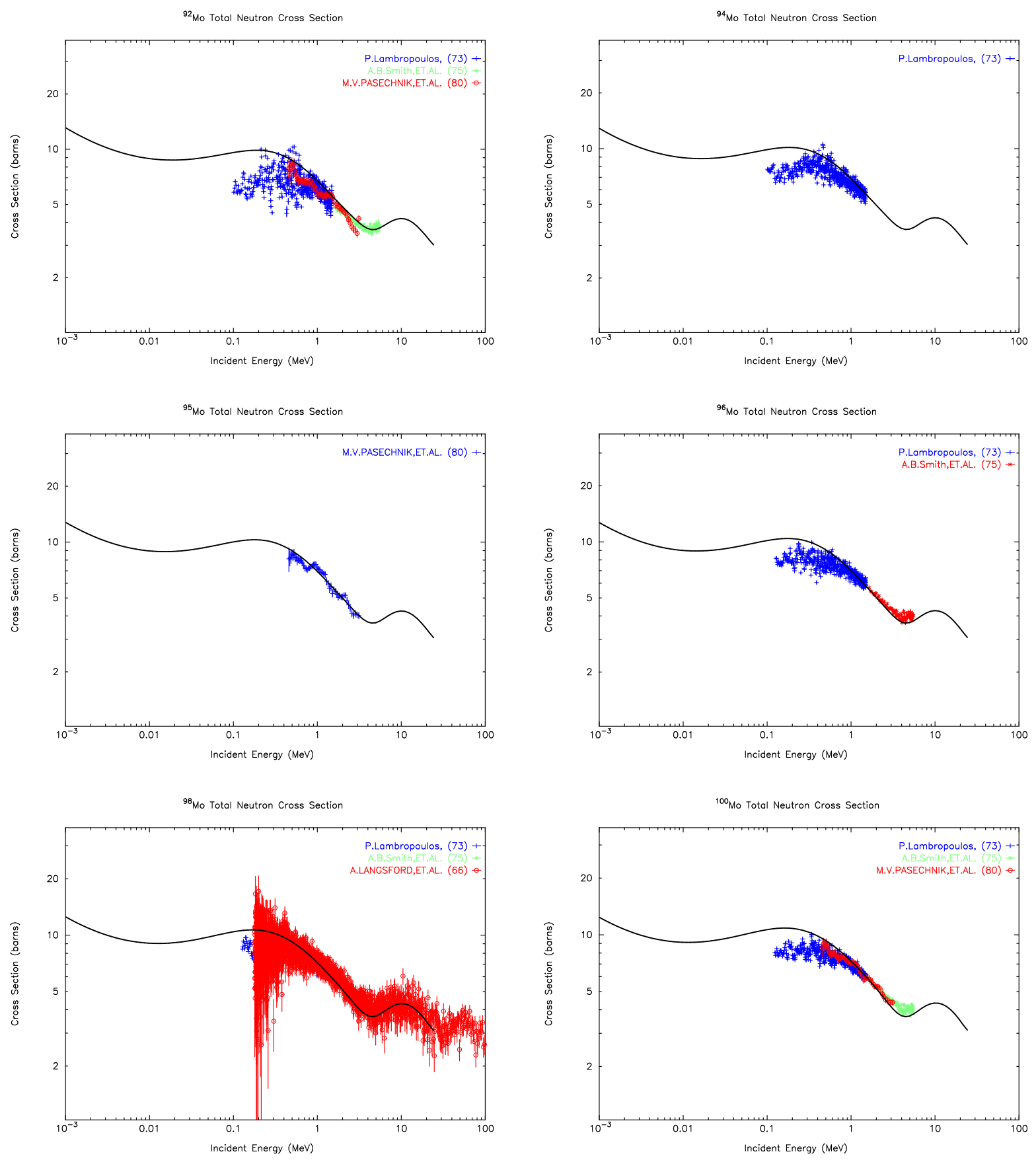

Fig. 1.- (continued)

(38 $\leq Z \leq 45$ ), we compare the total proton reaction cross section predicted by the optical model to measured values in Figure 3. In this figure, the black line represents the optical model calculation and the red data represents measurements taken from (EXFOR 2006). These comparisons indicate that the proton transmission coefficients for the

Koning and Delaroche optical potential may be somewhat high for the lighter targets considered in this study, though only by $10-15 \%$ at most at 14.5 MeV. A large proton transmission coefficient should only appreciably affect the calculated $(\mathrm{p}, \mathrm{n})$, $(\mathrm{p}, 2 \mathrm{n}),(\mathrm{n}, \mathrm{p})$, and $(\mathrm{n}, \mathrm{np})$ cross sections. 

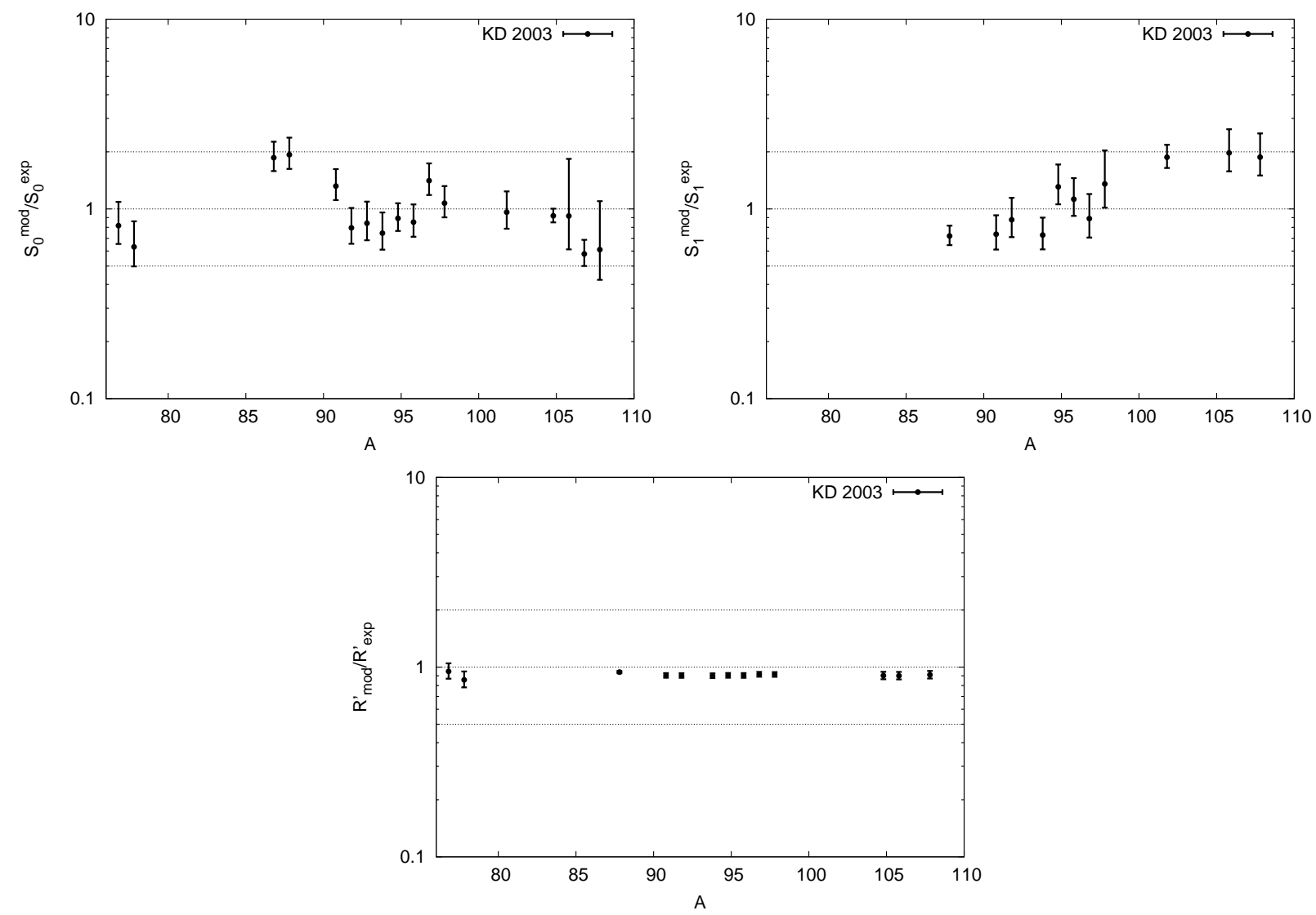

Fig. 2.- Measured s- and p-wave strength functions and mean scattering radii compared to the predictions of the Koning and Delaroche optical potential. The measured values for the s- and p-wave strength functions $\left(S_{0}\right.$ and $\left.S_{1}\right)$ are taken from (Belgya et al. 2005). Measured scattering radii are taken from (Mughabghab et al. 1981). Plotted are the ratios of the modeled quantities to their measured counterparts. The dotted lines indicate unity and factor of two deviations.

\subsubsection{The Alpha and Deuteron Optical Poten- tials}

We have included possible alpha and deuteron exit channels (and appropriate transmission coefficients) in this modeling effort. For these particles we use the well-established spherical optical potentials of (Avrigeanu et al. 1994) and (Lohr \& Haeberli 1974), respectively.

We do not include a quality analysis of these potentials in this report. This is primarily due to the lack of total cross section (and total reaction cross section) data in the local region at energies of interest in this study. For the majority of the reaction channels critical to the the radiochemical diagnostics, the deuteron and alpha particle exit channels are quite small when compared to the dominant exit channel, and hence errors introduced through the deuteron and alpha particle optical models should be insignificant. The only exceptions are the $(\mathrm{n}, \alpha)$ and $(\mathrm{n}, \mathrm{n} \alpha)$ reactions producing zirconium from stable molybdenum in the detector set Mo1278. Nonetheless, the degree to which our modeling effort is able to reproduce measured (n, $\alpha)$ and $(\mathrm{n}, \mathrm{n} \alpha)$ cross sections up to 15 $\mathrm{MeV}$ in the region of interest gives us some confidence in the alpha particle optical potential (see Appendices C.8 and C.9).

The deuteron exit channels are always very small compared to the dominant channel, and were not included in the previous RADCHEM detector sets. Incident deuteron reactions, particularly $(\mathrm{d}, 2 \mathrm{n})$ and $(\mathrm{d}, 3 \mathrm{n})$, are of importance in radiochemical diagnostics, though. The deuteron induced reactions of greatest importance have been measured, and will be used in lieu of modeled cross sections in the UGT analysis. 

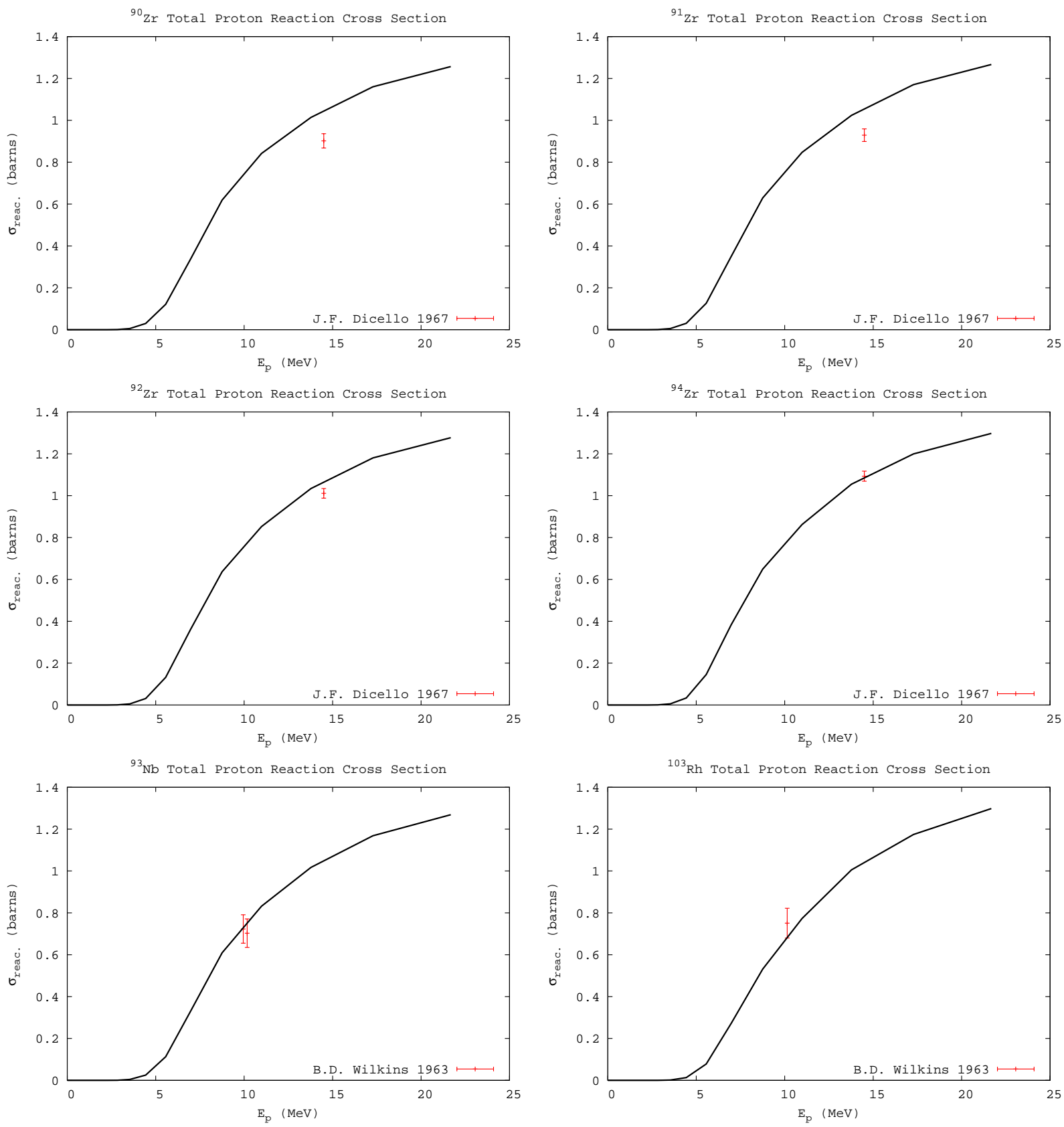

Fig. 3.- Total measured proton reaction cross sections vs those predicted by the optical model of KoningDelaroche for select targets in the range $38 \leq Z \leq 45$. Measured values were obtained from (EXFOR 2006). The optical model prediction is indicated by the solid black line.

\subsubsection{Transmission Coefficients for Photons}

Gamma ray transmission coefficients were calculated using a simple model which depends only on the multi-pole type (XL) and the transition energy $(\epsilon)$. They are related to the gamma ray strength function $f_{X L}^{\gamma}(\epsilon)$ by

$$
T_{X L}^{\gamma}(\epsilon)=2 \pi \epsilon^{2 L+1} f_{X L}^{\gamma}(\epsilon)
$$

The energy dependence of the strength function was determined using the GDR model with enhanced generalized Lorentzian (EGLO) line shapes (Kopecky et al. 1993). In particular, the E1 strength function is given by

$$
f_{E 1}^{\gamma}(\epsilon)=\mathcal{N} \frac{4}{3 \pi} \frac{e^{2}}{\hbar c} \frac{1}{M_{p} c^{2}} \times
$$




$$
\begin{gathered}
{\left[\frac{\epsilon \Gamma_{G D R}\left(\epsilon, T_{f}\right)}{\left(\epsilon^{2}-E_{G D R}^{2}\right)^{2}+\left(\Gamma_{G D R}\left(\epsilon, T_{f}\right) \epsilon\right)^{2}}+\right.} \\
\left.0.7 \frac{\Gamma_{G D R}\left(0, T_{f}\right)}{\epsilon^{3}}\right]
\end{gathered}
$$

where $M_{p}$ is the proton mass. The energy dependent width $\Gamma_{G D R}\left(\epsilon, T_{f}\right)$ is given by

$$
\begin{aligned}
\Gamma_{G D R}\left(\epsilon, T_{f}\right)= & {\left[\kappa+(1-\kappa) \frac{\epsilon-\epsilon}{E_{G D R}-\epsilon}\right] \times } \\
& \frac{\Gamma_{G D R}}{E_{G D R}^{2}}\left[\epsilon^{2}+\left(2 \pi T_{f}\right)^{2}\right]
\end{aligned}
$$

with $\epsilon=4.5 \mathrm{MeV}$. For nuclei with $A<148$, the factor $\kappa$ is unity. For heavier nuclei, $\kappa=1+$ $0.009(A-148)^{2} \exp [-0.18(A-148)]$. The $T_{f}$ that appears in Equations 6 and 7 is the temperature of the final state, determined from the level density parameters. For a backshifted transition energy $U=S_{n}-\epsilon-\Delta$ one first determines the energy dependent level density parameter $a$ (see Section 3.3.2). Provided $U$ is positive, the temperature is given by

$$
T_{f}=\frac{a}{2}[1+\sqrt{1+4 a U}]
$$

Otherwise, $T_{f}=1 / a$.

The parameters $E_{G D R}, \Gamma_{G D R}$, and $\sigma_{G D R}$ (the energy, width, and peak cross section of the GDR resonance) have been measured for several nuclei (Belgya et al. 2005). Based on these measurements, we have adopted a systematic description of these parameters for other targets. Using only measured GDR parameters from the local region of interest, we fit systematic values of the form $E_{G D R} \propto A^{-1 / 6}, \Gamma_{G D R} \propto A^{-1 / 3}$, and $\sigma_{G D R} \propto A$. As suggested in (Woosley et al. 1976), the GDR width of nuclei on or near closed shells will generally be lower than those predicted by such systematics. To account for these "shell effects", we divide the measured GDR widths of closed shell nuclei by a factor of 0.6 and those one nuclei away from a closed shell by a factor of 0.8 prior to fitting the systematics. The resulting systematic GDR widths for closed shell and near-closed shell nuclei are then multiplied by factors of 0.6 and 0.8 , respectively. Measured GDR parameters are used in preference to systematics where they are available. The resulting systematic fits are presented in Figures (4-6), and are given by

$$
\begin{aligned}
E_{G D R} & =\frac{34.3506 \mathrm{MeV}}{A^{1 / 6}} \\
\Gamma_{G D R} & =\frac{26.7114 \mathrm{MeV}}{A^{1 / 3}}
\end{aligned}
$$

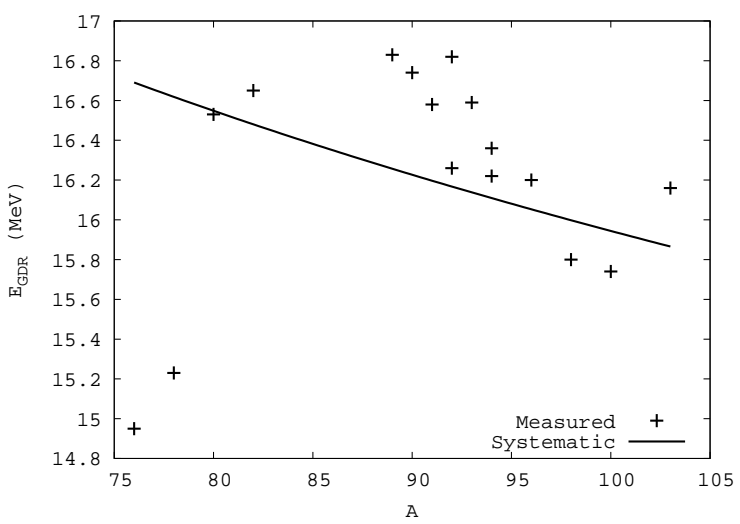

Fig. 4.- Systematics for the GDR energy. The measured data in the local region of interest are taken from (Belgya et al. 2005).

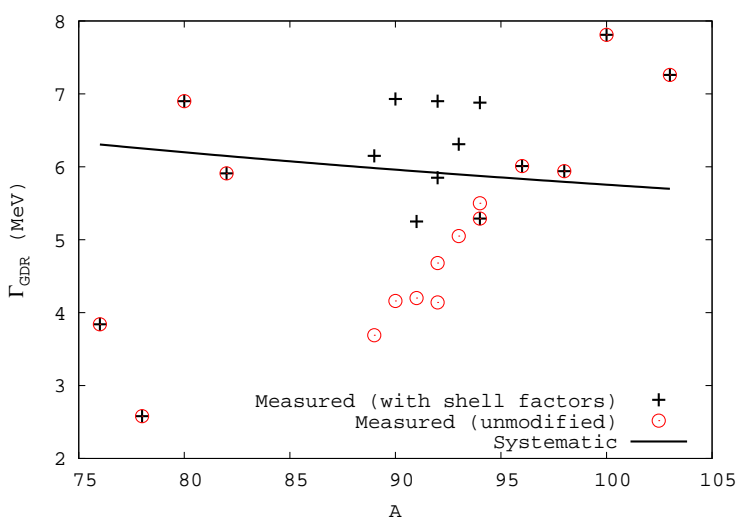

Fig. 5.- Systematics for the GDR width. The unmodified measured data in the local region of interest, indicated by the red circles, are taken from (Belgya et al. 2005). The modified data to which the systematic was fit, as described in the text, is indicated by the solid black markers.

$$
\sigma_{G D R}=A \times 1.81357 \mathrm{mb}
$$

where $A$ is the mass number of the compound nucleus. We consider only a single E1 resonance.

We also include M1, E2, M2, E3, and M3 transitions in our modeling. For the M1 strength function, we adopt a Simple Lorentzian (SLO) model

$$
\begin{array}{r}
f_{M 1}^{\gamma}(\epsilon)=\mathcal{N}_{M 1} \frac{4}{3 \pi} \frac{e^{2}}{\hbar c} \frac{1}{M_{p} c^{2}} \times \\
\frac{\epsilon \Gamma_{G D R}}{\left(\epsilon^{2}-E_{G D R}^{2}\right)^{2}+\left(\Gamma_{G D R} \epsilon\right)^{2}}
\end{array}
$$

with the global set of GDR parameters given in (Belgya et al. 2005):

$$
E_{G D R}=\frac{41}{A^{1 / 3}}
$$




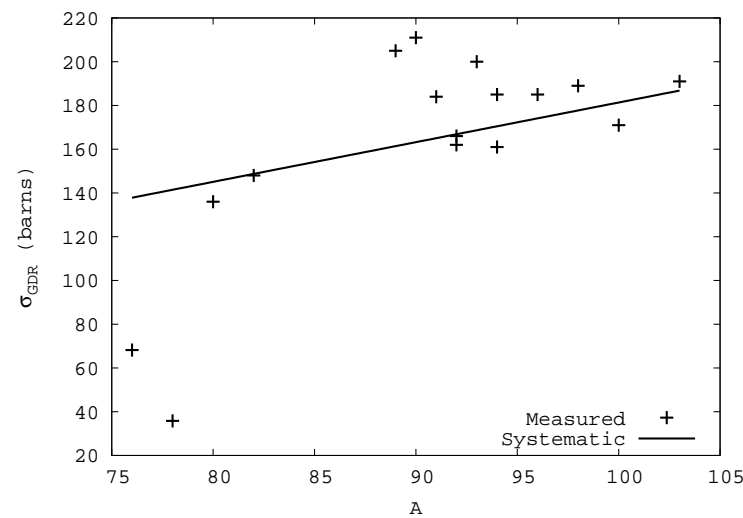

Fig. 6.- Systematics for the GDR peak cross section. The measured data in the local region of interest are taken from (Belgya et al. 2005).

$$
\Gamma_{G D R}=4
$$

The overall normalization for the M1 strength function $\left(\mathcal{N}_{M 1}\right)$ is determined such that

$$
\frac{f_{E 1}\left(S_{n}\right)}{f_{M 1}\left(S_{n}\right)}=0.0588 \cdot A^{0.878}
$$

where $S_{n}$ is the neutron separation energy.

The remaining transmission coefficients are simply proportional to $\epsilon^{2 L+1}$, i.e. their strength functions are constants. In particular,

$$
\begin{aligned}
f_{E 2}^{\gamma}(\epsilon) & =7.2 \times 10^{-7} A_{C}^{2 / 3} f_{E 1}^{\gamma}\left(S_{n}\right) \\
f_{M 2}^{\gamma}(\epsilon) & =2.2 \times 10^{-7} f_{E 1}^{\gamma}\left(S_{n}\right) \\
f_{E 3}^{\gamma}(\epsilon) & =3.4 \times 10^{-13} A_{C}^{4 / 3} f_{E 1}^{\gamma}\left(S_{n}\right) \\
f_{M 3}^{\gamma}(\epsilon) & =1.1 \times 10^{-13} A_{C}^{2 / 3} f_{E 1}^{\gamma}\left(S_{n}\right)
\end{aligned}
$$

The factor $\mathcal{N}$ appearing in equation 6 is an overall normalization constant, determined by fitting the average total s-wave radiation width at the neutron binding energy,

$$
\begin{gathered}
\left\langle\Gamma_{\gamma}\right\rangle_{0}=\frac{J+1}{2 J+1}\left\langle\Gamma_{\gamma}\left(B_{n}, J+\frac{1}{2}\right)\right\rangle \\
+\frac{J}{2 J+1}\left\langle\Gamma_{\gamma}\left(B_{n}, J-\frac{1}{2}\right)\right\rangle \\
\Gamma_{\gamma}(E, J)=\frac{T_{\gamma}(E, J)}{2 \pi \rho(E, J)}(\mathrm{meV})
\end{gathered}
$$

(Uhl \& Strohmaier 1976). Here, $J$ is the spin of the target nucleus. The gamma-ray transmission coefficients are evaluated as in Equation 3 with the summation over multipoles instead of spins and parities.
Since the total s-wave radiation width is generally measured only for stable isotopes plus a neutron, we have developed a systematic approach for estimating this value for the many unstable nuclei in our region of interest. Systematic descriptions of the average total s-wave radiation width generally exhibit a dependence on the mass and s-wave resonance spacing $\left(D_{0}\right)$ of the nucleus (Gardner 1975). We find that the measured radiation widths from (Belgya et al. 2005) are generally well fit by a plane in the $\left(A, \log _{10} D_{0}\right)$ coordinate space:

$$
\begin{aligned}
\left\langle\Gamma_{\gamma}\right\rangle_{0}^{\text {sys }}= & (251.536 A-14590.7) \\
& \times\left(\log _{10} D_{0}-8.10272\right)
\end{aligned}
$$

This systematic is shown in Figure (7). Whenever they are available, we use measured radiation widths instead of systematics.

\subsection{Nuclear Level Densities}

\subsubsection{Level Density Models}

Another important input to the statistical model code is the nuclear level density. For this project, we have adopted a standardized, semiempirical approach which is numerically efficient, can be tied to experimental data, and is fairly accurate. The level density is described by two functions. Both are energy dependent, and the second factor contains the spin dependence. This is the "Back-shifted Fermi Gas" formulation of the nuclear level density:

$$
\rho(U, J)=\rho(U) f(U, J)
$$

where $\rho(U)$ is the state density, with $U=E-\Delta$ the back-shifted energy. $\Delta$ is the so called "backshift", and $J$ is the spin of the compound nucleus. It is assumed that the parity distribution of nuclear states is equal, i.e. for a given parity $\Pi$ $\rho(U, J, \Pi)=\frac{1}{2} \rho(U, J)$. We will further treat each of these in two ways, depending on the excitation energy of interest. The demarcation point will be roughly between the energy range of the known excited levels of a given compound nucleus (the low energy domain), and near (and above) the neutron binding energy (the high energy domain).

For the high energy domain, we describe the level density assuming a Fermi gas formula,

$$
\begin{array}{r}
\rho(U)=\frac{\sqrt{\pi}}{12} \frac{\exp (2 \sqrt{a U})}{a^{1 / 4} U^{5 / 4}} \frac{1}{\sqrt{2 \pi} \sigma} \\
f(U, J)=\frac{2 J+1}{2 \sigma^{2}} \exp \left[\frac{-\left(J+\frac{1}{2}\right)^{2}}{2 \sigma^{2}}\right]
\end{array}
$$



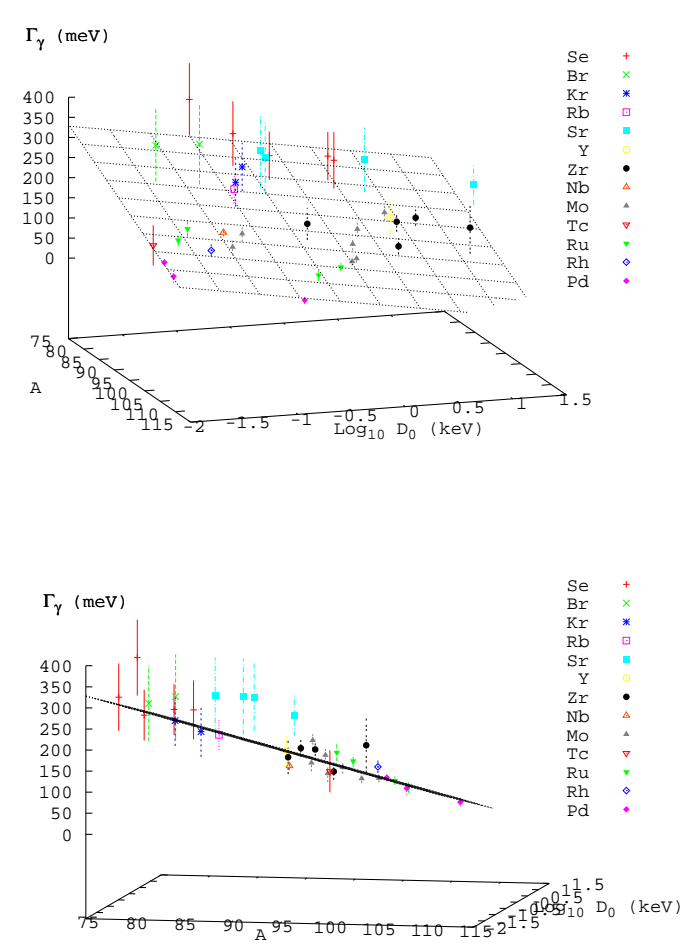

Fig. 7.- Systematics for average total s-wave radiation widths. The systematic is presented from two perspectives. The upper pane shows the relative mass and resonance spacing of the nuclei with measured radiation widths. The lower pane show the same systematic, only looking directly down the systematic plane to illustrate deviations of the systematic from measured values.

where $a(E)$ is the level density parameter (in $\left.\mathrm{MeV}^{-1}\right)$. The spin cutoff parameter $\sigma^{2}$ is defined as

$$
\sigma^{2}=0.01496 \lambda A^{5 / 3} \sqrt{\frac{U}{a}}
$$

The level density assumes an equal distribution of parity states. Note that at low excitation energy (for a positive back-shift), Eq. (17) diverges. At low energies, the nuclear level density is better described by a constant temperature formula:

$$
\rho(E) \propto \exp \frac{E-E_{0}}{T}
$$

The level density parameters can be calculated using experimental data. For the Fermi-gas state density (Eq. 17), the level density parameter, $a(E)$, can be related to the average level spacing $\left(D_{0}\right)$ near the neutron binding energy. The back- shift $\Delta$ is calculated as a difference in binding energies of adjacent nuclei (Bohr \& Mottelson). The constant temperature parameters $E_{0}$ and $T$ are fixed by the choice of a matching energy $E_{x}$ (chosen such that the state density that goes through the low lying spectroscopic levels) and the condition that the two state densities match tangentially at $E_{x}$. We describe below how we determined these parameters for all of the nuclei considered in this study.

\subsubsection{Fermi-Gas Level Densities}

Our goal is to fit the level density parameter $a$ in Eq. (17) to experimental data where available. We adopt an energy dependent form, $a(U, Z, N)$, and begin by fixing the spin cutoff parameter and the pairing energies.

\section{The Spin Cutoff Parameter}

The spin cutoff parameter $\sigma^{2}$, Eq. (19), characterizes the spin distribution of the Fermi gas level density. It depends on the parameters $a$, the level density parameter, and $\lambda$, which determines the effective moment of inertia for the nucleus in question. In principle it could be determined by experiment, for example, by comparing ratios of cross sections leading to different isomers of the product nucleus (Keisch 1963). Because data like this is often sparse, especially in the limited regions of the periodic chart we are interested in, and because we are often interested in reactions that proceed on or through radioactive species where no such data exists, we must resort to models. In our analysis, we fix $\lambda=1$ in Eq. (19), corresponding to the moment of inertia of a rigid sphere.

\section{Pairing Energies}

In determining the backshift $\Delta$, also known as the pairing energy, we use the method of (Rauscher et al. 1997). The total pairing energy is equal to the sum of the proton and neutron pairing energies

$$
\begin{aligned}
\Delta(Z, N) & =\frac{1}{2}\left(\Delta_{p}+\Delta_{n}\right) \\
\Delta_{p}(Z, N) & =E^{G}(Z, N) \\
& -\frac{1}{2} E^{G}(Z-1, N) \\
& -\frac{1}{2} E^{G}(Z+1, N) \\
\Delta_{n}(Z, N) & =E^{G}(Z, N)
\end{aligned}
$$




$$
\begin{aligned}
& -\frac{1}{2} E^{G}(Z, N-1) \\
& -\frac{1}{2} E^{G}(Z, N+1)
\end{aligned}
$$

where $E^{G}(Z, N)$ is the binding energy of the nucleus $(Z, N)$.

\section{The Level Density Parameter}

At high energies, the level density parameter $a$ behaves essentially as a function of mass number only. However, it has been shown (Iljinov et al. 1992) that at low energies it is more appropriate to use an energy dependent form:

$$
a(U, Z, N)=\tilde{a}(A)\left[1+\delta W(Z, N) \frac{f(U)}{U}\right]
$$

with

$$
f(U)=1-\exp (-\gamma U)
$$

and as usual $U=E-\Delta$.

In previous works where a more global prescription was developed [(Rauscher et al. 1997), (Iljinov et al. 1992)], one would adopt a semiempirical shell correction, $\delta W(Z, N)$, and fit $\tilde{a}(A)$ to known experimental data. Here we choose to adopt a simple form for the mass dependent term and fit the shell correction. In our analysis, we followed the convention of (Rauscher et al. 1997) in choosing the parameters $\gamma=0.04884$ and $\tilde{a}=$ $0.1337 A-0.06571 A^{2 / 3}$.

\section{Shell Corrections}

Given an asymptotic value of the level density parameter, the shell corrections can be determined for select nuclei from experimental values of the average level spacings $D_{0}$ as determined by neutron resonance analysis (Belgya et al. 2005). For $s$-wave resonances (neutron angular momentum equal to zero), the calculated level spacing, $D_{\text {calc }}$, evaluated at the neutron binding energy $U=$ $B_{n}-\Delta$, is related to the nuclear level density (e.g. Equations 16-18)

$$
D_{\text {calc }}=\frac{2}{\rho\left(U, J=\frac{1}{2}\right)}
$$

for nuclei with spin $s=0$ and

$$
D_{\text {calc }}=\frac{2}{\rho\left(U, J=s+\frac{1}{2}\right)+\rho\left(U, J=s-\frac{1}{2}\right)}
$$

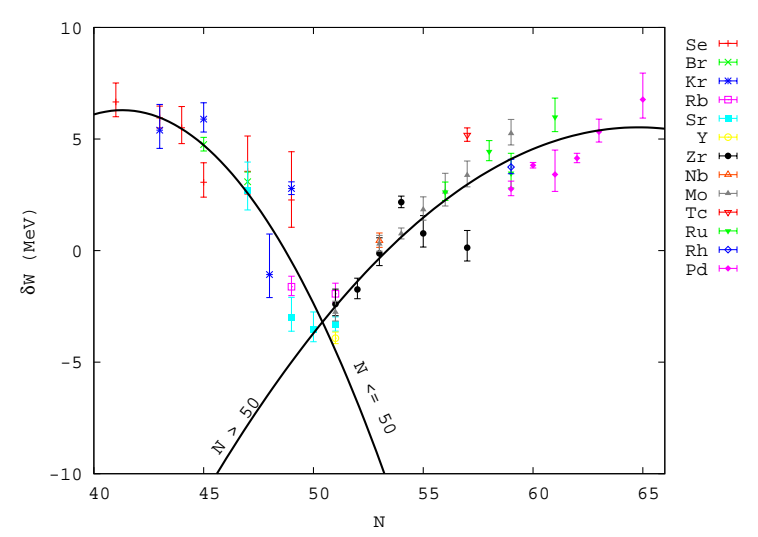

Fig. 8. $-\chi^{2}$ fit to experimentally determined shell corrections, used to systematically determine unknown shell corrections.

for nuclei with $s \neq 0$. In each case, the level densities $\rho(U, J)$ were calculated using the other parameters $(\lambda, \Delta, \gamma, \tilde{a}(A))$ set as previously described. We then numerically solved for the value of $\delta W$ that would minimize the quantity $D_{\text {calc }}-$ $D_{0}$ using root bisection methods.

There are only a limited number of nuclei for which the average resonance spacing $D_{0}$ has been measured (i.e. for compound nuclei formed from a stable target plus a neutron). As a result, we were required to systematically predict the shell correction for the remaining nuclei in the range of interest. After plotting the "experimental" $\delta W$ in our region of interest and their associated errors we noted two segments with a roughly quadratic behavior. A $\chi^{2}$ fit to the data results in the systematic

$$
\begin{array}{ll}
N \leq 50 & : \quad \delta W=-0.114 N^{2}+9.45 N-188.8 \\
N>50 & : \quad \delta W=-0.042 N^{2}+5.46 N-171.3
\end{array}
$$

This systematic, along with the derived "experimental" shell corrections, is shown in Figure 8. Of course, where available, we always use an experimentally determined shell correction over a systematic one.

\subsubsection{Constant Temperature Level Density}

For the lower energy regions, below the neutron binding energy $B_{n}$, the nuclear level density has the same formulation as Equation (16). However, particularly at and below the pairing energy $\Delta$, the state density in Equation (17) becomes imaginary. Unfortunately, experimental level schemes are rarely known above $2 \mathrm{MeV}$ of excitation en- 
ergy. In practice we are forced again to assume a model and use all available experimental data to constrain its parameters.

Of course the two prescriptions for the level density must match at some energy intermediate to where they are constrained by experiment. Henceforth we will refer to the high energy level density as $\rho_{1}$, and the low energy density as $\rho_{2}$.

Gilbert and Cameron (Gilbert \& Cameron 1965) noticed that the cumulative number of observed levels (the so-called staircase plot, which increase exponentially), can be fit with straight lines in a semi-log plot. They adopted a constant temperature formula to fit these:

$$
N(E)=\exp \left[\frac{E-E_{0}}{T}\right]
$$

with $N(E)$ being the cumulative number of levels at excitation energy $E, E_{0}$ and $T$ are two free parameters to be fit to the observed level structure. The observable level density is given by

$$
\rho_{2}(E)=\frac{d N(E)}{d E}=\frac{1}{T} \exp \left[\frac{E-E_{0}}{T}\right]
$$

where $T$ now takes on the meaning of a nuclear temperature which is constant in the region of the discrete levels. We assume that Eq. (27) can be extrapolated from the region of the known discrete levels to higher energies, where the Fermi-gas level density $\left(\rho_{1}\right)$ is valid. We then define the notion of a fit to the total level density over the entire range as being achieved if: (a) a good fit can be made to the low lying levels, (b) the observed level spacing at the neutron binding energy is exactly reproduced, and (c) the energy of the matching point $E_{x}$ for the two prescriptions falls between $E=\Delta$ and $E=B_{n}$, and that they match at this point with the same slope, i.e. for $E=E_{x}$ :

$$
\begin{aligned}
\rho_{1}\left(E_{x}\right) & =\rho_{2}\left(E_{x}\right) \\
\frac{d \log \rho_{1}\left(E_{x}\right)}{d E} & =\frac{d \log \rho_{2}\left(E_{x}\right)}{d E}
\end{aligned}
$$

From the first of these, we can determine $E_{0}$ :

$$
E_{0}=E_{x}-T \log T \rho_{1}\left(U_{x}\right)
$$

where $U_{x}=E_{x}-\Delta$. The second condition can be satisfied by assuming that at $E_{x}$ the constant nuclear temperature $T$ of the low lying states is equal to the energy dependent nuclear temperature $\tau\left(U_{x}\right)$ of the high excited states,

$$
\begin{aligned}
\frac{1}{T} & =\sqrt{\frac{a}{U_{x}}}-\frac{3}{2 U_{x}} \\
+ & \frac{(\tilde{a}-a)\left(1+\gamma U_{x}\right)+\tilde{a} \gamma \delta W}{\sqrt{a U_{x}}}
\end{aligned}
$$

where $a$ is given by Eq. (22). If there is no shell correction, the latter term in the above equation is zero. Typical values for the matching energy are $2 \leq E_{x} \leq 8 \mathrm{MeV}$, and are approximated by $E_{x}=2.5+\frac{150}{A}+\Delta$ (Gilbert \& Cameron 1965). However, we fit the spectroscopic levels for each nucleus manually, adjusting $E_{x}$ to give the best possible fit.

Occasionally when fitting $E_{x}$ to the spectroscopic discrete levels, one encounters a situation where the ideal $E_{x}$ either lies below the backshift (so that the Fermi gas portion of the level density diverges) or results in a negative temperature when $E_{0}$ and $T$ are determined by the tangential match at $E_{x}$. We prefer to avoid such unphysical level densities. Usually, these situations occur where the cumulative number of levels (i.e. the "staircase plot") is not well fit by a constant temperature formula because levels are likely missing from the scheme. In these cases, we reduce the number of levels fit until they are well described by a constant temperature formula, and adjust $E_{x}$ to get the best match possible. We also make an extra effort to fit the level density to the highest known discrete level included in the level scheme, so that the effective level density used in the Hauser-Feshbach calculations will be continuous between energies where the discrete levels and level densities are used.

We present in Figure (9) a sample of constant temperature fits to low lying levels. In these figures, the blue lines represent the cumulative number of levels for which spins and parities are known. The green lines represent additional levels above the point where the level scheme is complete. Red lines represent Fermi gas level densities, and magenta lines represent constant temperature level densities. Red circles indicate the value of the matching energy $E_{x}$. The larger plot shows the the fit compared to the levels used in our Hauser-Feshbach calculation. The smaller insets show the fit compared to all available levels, including those above the point where the level scheme is considered complete.

\section{Behavior of the Spin Cutoff Parameter Below $E_{x}$}

At the matching energy $E_{x}$, the spin cutoff parameter is given by Equation (19). For energies below $E_{x}$, we assume $\sigma^{2}=\sigma^{2}\left(E_{x}\right)$.

Other treatments have been suggested for the behavior of $\sigma^{2}$ below $E_{x}$. One may define $E_{c u t}$ as the energy of the highest known excited level for 

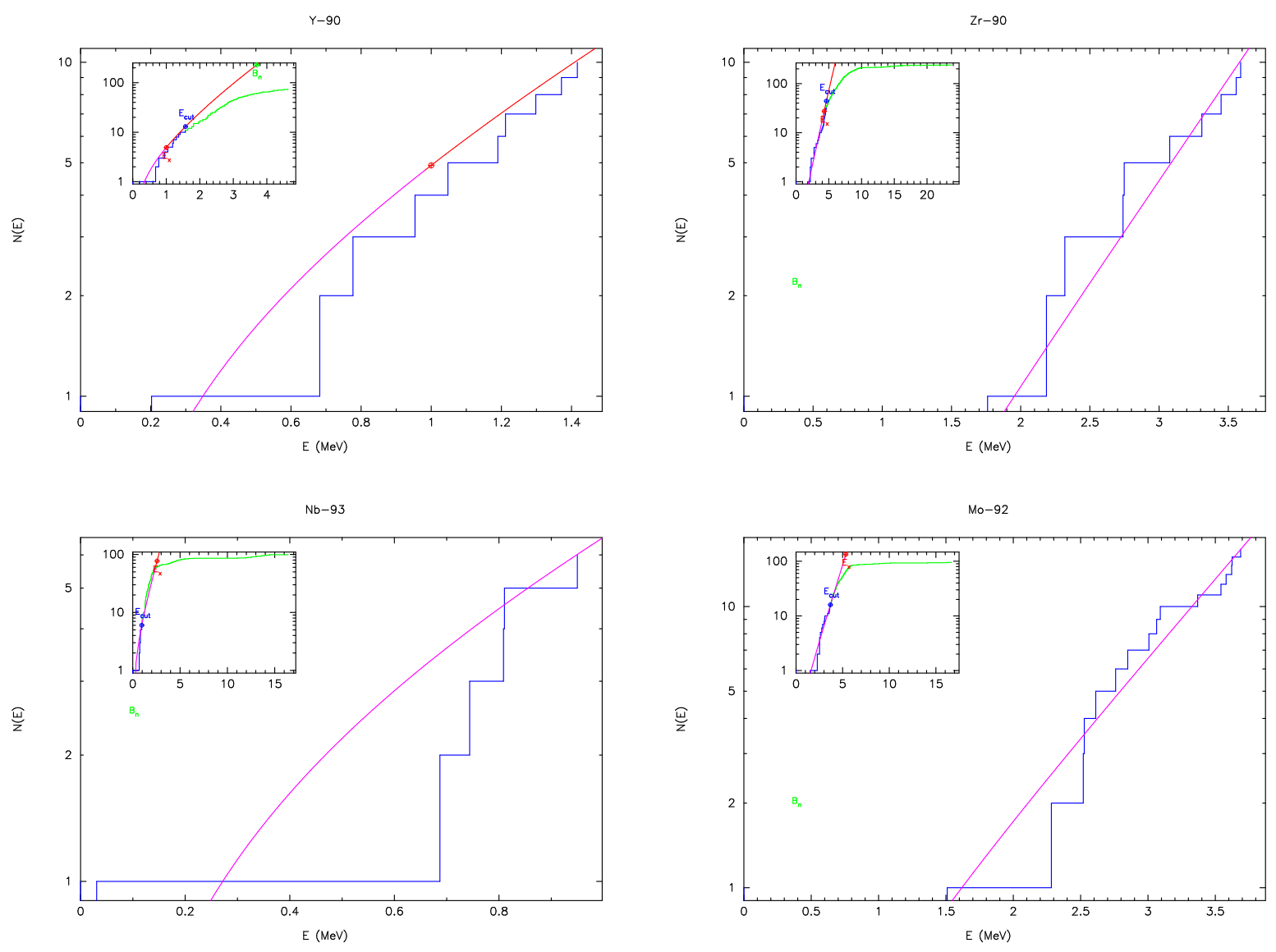

Fig. 9.- Select constant temperature level density fits to the low lying spectroscopic levels. See text for details.

which energy, spin and parity are explicitly known. Using the known spectroscopic levels, a low energy spin cutoff parameter at $E_{c u t}$ may be defined:

$$
\sigma_{E_{\text {cut }}}^{2}=\frac{1}{2 N} \sum_{i=1}^{N}\left(J_{i}+\frac{1}{2}\right)^{2}
$$

where $\mathrm{N}$ represents the number of the level with energy $E_{c u t}, J_{i}$ are the spins of the individual levels. The sum excludes the ground state $(\mathrm{i}=0)$. This value is used for energies $0 \leq E \leq E_{\text {cut }}$. For energies $E_{\text {cut }} \leq E \leq E_{x}$, the spin cutoff parameter is given by a linear fit between these two values (Equations (19) and (33)). Specifically,

$$
\sigma_{I}^{2}=\sigma_{E_{x}}^{2}-\frac{\sigma_{E_{x}}^{2}-\sigma_{E_{c u t}}^{2}}{E_{x}-E_{c u t}}\left(E_{x}-E\right)
$$

This conforms to the treatment of $\sigma^{2}$ in the IDA reaction code system (Reffo 1978). This particular treatment works well when there are a reasonably large number of levels to fit.
Another treatment, used in the GNASH code system (Chadwick 1998), defines:

$$
\begin{aligned}
\sigma_{H}^{2} & =\sigma_{E_{x}}^{2} \\
U_{L} & =\max \left(E_{c u t}-\Delta, 0.1\right) \\
\sigma_{L}^{2} & =\lambda \sqrt{a U_{L}} A^{2 / 3} \\
\sigma_{G}^{2} & =\sigma_{L}^{2}+\frac{E-\frac{1}{2} E_{c u t}}{E_{x}-\frac{1}{2} E_{c u t}}\left(\sigma_{H}^{2}-\sigma_{L}^{2}\right)
\end{aligned}
$$

The form $\sigma_{G}^{2}$ is then used between $\frac{1}{2} E_{c u t}$ and $E_{x}$.

The behavior of $\sigma^{2}$ below $E_{x}$ will only affect the level density used in Hauser-Feshbach calculations between $E_{c u t}$ and $E_{x}$, since the discrete levels are accounted for individually. The changes that arise in the level density between $E_{c u t}$ and $E_{x}$ due to the choice of how $\sigma$ is treated in this range are generally small.

The fitted parameters for the total level density are presented in Appendix B.4. The symbols in the legend are the same as described above. In column five, an " $\mathrm{x}$ " indicates the shell correction 
$\delta W$ was derived from an experimentally known level spacing $D_{0}$, an "s" indicates the shell correction was derived from the systematic shown in Figure 8. The last column indicates the number of excited states included in the fit.

\subsection{Pre-Equilibrium Model Parameters}

The exciton model described in section 2.4 has two free parameters which can be tuned to best reproduce measured cross sections in conjunction with the other statistical model inputs. The first is the constant $\langle F M\rangle$ which scales the average effective matrix element of the residual interactions. Increasing this parameter enhances the particle emission rates and, subsequently, the fraction of the reaction cross section involved in preequilibrium processes. We have found that a value of $\langle F M\rangle=250 \mathrm{MeV}$ is optimal for reproducing measured $(\mathrm{n}, 2 \mathrm{n}),(\mathrm{n}, \mathrm{p})$, and $(\mathrm{p}, \mathrm{n})$ cross sections in this region. Radiative neutron capture reactions, being very small at incident energies where pre-equilibrium processes are important, exhibit no sensitivity to this parameter.

The other tunable parameter, $\phi$, describes the probability of alpha cluster preformation. It follows that an increase in this variable will lead to an enhancement of alpha particle emission during the pre-equilibrium phase. However, since alpha emission is small compared to nucleon emission, modifying $\phi$ will have negligible effects on cross sections that do not involve alpha particles in the exit channel. We may thus use the $(\mathrm{n}, \alpha)$ reaction to tune this parameter, for which we find a value of $\phi=0.20$ to give satisfactory results. Previous works suggest that for compound nuclei of various masses this parameter will generally fall in the range $0.1 \leq \phi \leq 0.8$ (Milazzo-Colli \& Braga-Marcazzan 1973).

We assume an initial two-particle one-hole configuration for the exciton model. In calculating emission rates we use the nuclear level densities developed in section 3.3.

\section{Modeled Cross Sections}

\subsection{Comparison to Measured Cross Sec- tions}

Having developed the various input quantities based on available experimental data in the previous section, we now turn to the results of the STAPRE-H95 model and compare to available measured cross sections in the region of interest. We restrict our attention primarily to ground state targets of ${ }^{89} \mathrm{Y},{ }^{90} \mathrm{Zr},{ }^{93} \mathrm{Nb}$, and ${ }^{92,94} \mathrm{Mo}$ (stable loaded detector elements closest in mass to the measured radioactivities). Comparisons to other measured cross sections are provided in the appendices.

\subsubsection{Comparison to experimental $(n, \gamma)$ capture cross sections}

In Figure 10 we present comparisons for neutron capture reactions on select targets. Shown is the activation cross section (solid black lines in all plots that follow) defined as the sum of emission (both particle emission and gamma-ray cascade) from the compound nucleus that eventually leads to the ground state of the product (final) nucleus. We also provide (where appropriate) separate cross sections that decay to the ground state (red lines), and any long lived isomer (blue and green lines, see Appendix A.2 for a list of the isomers and their respective half-lives). These cross sections are plotted against the available experimental data, taken from the Experimental Nuclear Reaction Data File (EXFOR 2006). Cross sections for the total, ground, and isomeric states are colored in a similar manner to the modeled cross sections (gray is activation, orange is to ground, and light blue and green to an isomer, respectively), with different symbols distinguishing results from various experiments.

Our results are in excellent agreement with the wealth of measured data for ${ }^{89} \mathrm{Y}(\mathrm{n}, \gamma){ }^{90} \mathrm{Y}$ and ${ }^{93} \mathrm{Nb}(\mathrm{n}, \gamma){ }^{94} \mathrm{Nb}$. The only general exception is around $14 \mathrm{MeV}$ incident energy, where direct capture mechanisms (not included in our calculations) begin to become significant. Since the neutron capture cross section is usually two or three orders of magnitude smaller than the dominant neutron induced reactions at these energies, neglecting direct capture will introduce negligible errors into any network calculations. For ${ }^{90} \mathrm{Zr}(\mathrm{n}, \gamma){ }^{91} \mathrm{Zr}$, our calculation is high by roughly a factor of 1.5 between $\sim 5-50 \mathrm{keV}$. Below $5 \mathrm{keV}$, the effects of individual resonances (which cannot be reproduced with the statistical model) are present. For ${ }^{94} \mathrm{Mo}(\mathrm{n}, \gamma){ }^{95} \mathrm{Mo}$ our calculation is in excellent agreement with the single measured data point.

Additional comparisons for neutron capture cross sections are presented in Appendix C.1. A similar degree of agreement between our calculations and experiment is found in these comparisons (though our calculation appears to be high for ${ }^{87} \mathrm{Sr}(\mathrm{n}, \gamma){ }^{88} \mathrm{Sr}$ and ${ }^{99} \mathrm{Tc}(\mathrm{n}, \gamma){ }^{100} \mathrm{Tc}$, and a bit low for $\left.{ }^{96} \mathrm{Ru}(\mathrm{n}, \gamma){ }^{97} \mathrm{Ru}\right)$. Such results are con- 

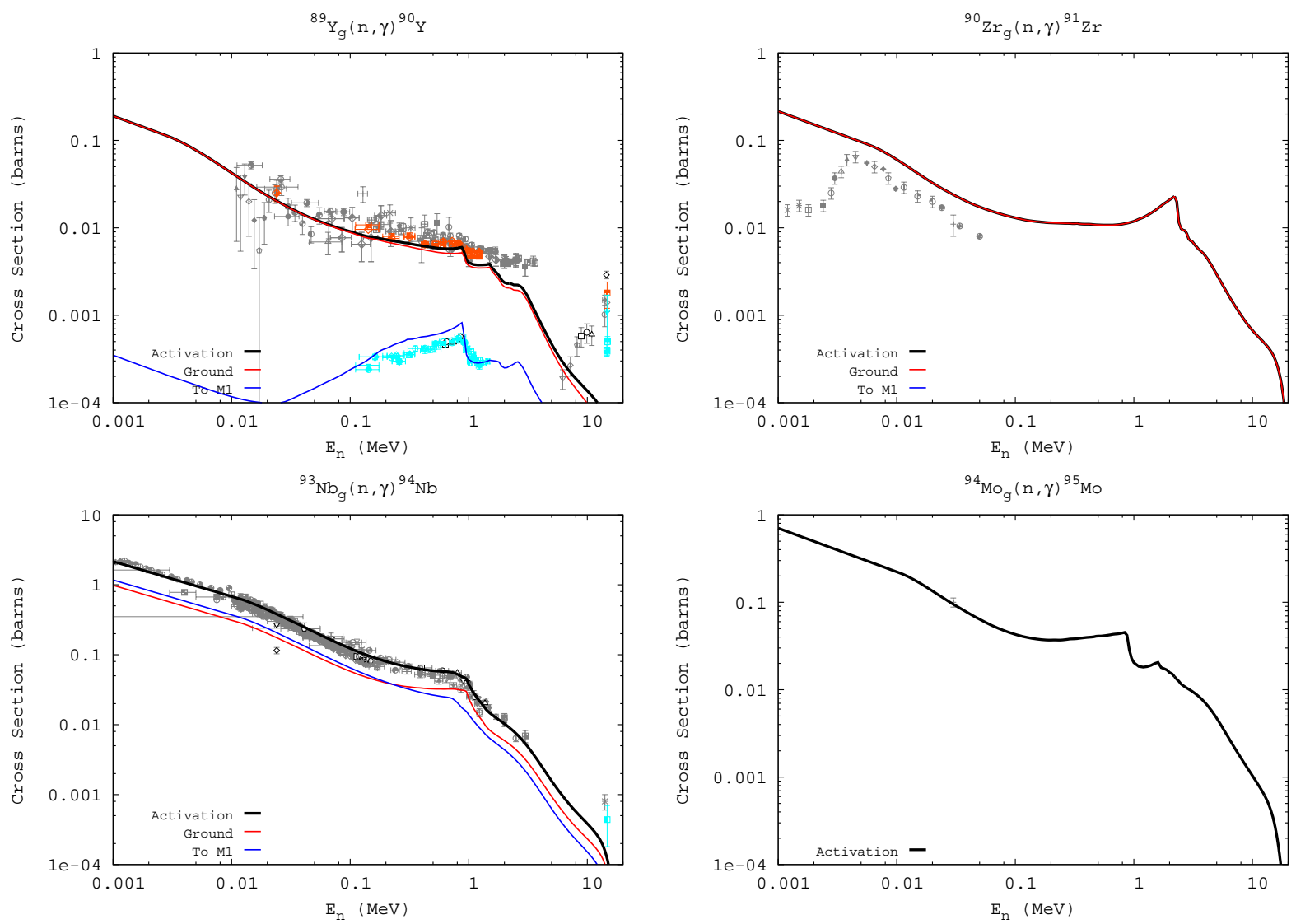

Fig. 10.- Calculated vs. measured $(\mathrm{n}, \gamma)$ cross sections on select stable isotopes in the region of interest. The data is taken from (EXFOR 2006). The black, red, and blue solid lines represent our modeled cross sections (total, leading to the ground state, and leading to the first isomer, respectively). The Gery, orange, and light blue data points are measured cross section data (total, ground state, and first isomer).

sidered good for $(\mathrm{n}, \gamma)$ activation cross sections. Using global systematics, $(\mathrm{n}, \gamma)$ cross sections can typically be modeled within a factor of two, often to within 30\% (Hoffman et al. 1999).

A quantitative comparison of our calculated cross sections to the experimental data shown in Figures 10 and 25 is given in Table 1 . For $(\mathrm{n}, \gamma)$ reactions we restrict our analysis to data with incident neutron energies of $30 \pm 2 \mathrm{keV}$. For each target listed in column (1), the subsequent column entries identify: (2) Res., the state of the residual (product) nucleus (activation, ground state, isomer); (3) $\mathrm{N}$, the number of experimental data points falling within the incident energy range; (4) $\bar{\sigma}$, the weighted average of the measured data (in barns), with weights corresponding to the inverse geometric mean of the errors in cross section and energy (i.e. $\left.w_{i}=\left(d E^{2}+d \sigma^{2}\right)^{-1 / 2}\right)$; (5) Dev., the standard deviation from the mean (also in barns), which gives an indication of the spread in the ex- perimental data; (6) $\sigma_{\text {mod. }}$, our modeled capture cross section (in barns); and ( 7$) \bar{\sigma} / \sigma_{\text {mod. }}$, the ratio of the weighted average of the data to our modeled value, which may be used as a scaling factor to be applied to a given cross section to bring it into conformity with its respective average experimental value. Also provided at the bottom of the table is an average absolute percent error (defined as $\left.\% E=N^{-1} \sum_{i=1}^{N}\left(\left|\sigma_{i}^{\text {mod. }}-\bar{\sigma}_{i}\right| / \bar{\sigma}_{i}\right) \times 100 \%\right)$ for all capture cross sections and the activation cross sections only.

On average, our modeled cross sections are within $35 \%$ of the weighted mean at $30 \mathrm{keV}$. Our activation cross sections are within $30 \%$ on average.

\subsubsection{Comparison to Maxwellian averaged $(n, \gamma)$ capture cross sections}

Yet another comparison to experimental data comes from the extensive efforts to measure and 
Table 1: Comparison of our modeled $(\mathrm{n}, \gamma)$ cross sections to experimental data at $30 \pm 2 \mathrm{keV}$

\begin{tabular}{|c|c|c|c|c|c|c|}
\hline${ }^{{ }^{A} Z}$ & Res. & $\mathrm{N}$ & $\bar{\sigma}$ & Dev. & $\sigma_{\text {mod }}$ & $\bar{\sigma} / \sigma_{\text {mod }}$ \\
\hline${ }^{84} \mathrm{Sr}$ & A & 1 & 0.470 & 0.000 & 0.426 & 1.103 \\
\hline${ }^{86} \mathrm{Sr}$ & M1 & 1 & 0.122 & 0.000 & 0.051 & 2.397 \\
\hline${ }^{86} \mathrm{Sr}$ & A & 21 & 0.097 & 0.061 & 0.068 & 1.434 \\
\hline${ }^{87} \mathrm{Sr}$ & A & 21 & 0.089 & 0.026 & 0.182 & 0.491 \\
\hline${ }^{89} \mathrm{Y}$ & A & 6 & 0.024 & 0.008 & 0.017 & 1.360 \\
\hline${ }^{90} \mathrm{Zr}$ & A & 1 & 0.011 & 0.000 & 0.024 & 0.459 \\
\hline${ }^{91} \mathrm{Zr}$ & A & 2 & 0.064 & 0.006 & 0.061 & 1.043 \\
\hline${ }^{92} \mathrm{Zr}$ & A & 1 & 0.034 & 0.000 & 0.039 & 0.871 \\
\hline${ }^{94} \mathrm{Zr}$ & A & 3 & 0.021 & 0.003 & 0.025 & 0.838 \\
\hline${ }^{96} \mathrm{Zr}$ & A & 2 & 0.022 & 0.014 & 0.016 & 1.366 \\
\hline${ }^{93} \mathrm{Nb}$ & A & 18 & 0.281 & 0.030 & 0.319 & 0.882 \\
\hline${ }^{94} \mathrm{Mo}$ & A & 1 & 0.100 & 0.000 & 0.095 & 1.052 \\
\hline${ }^{95} \mathrm{Mo}$ & A & 1 & 0.440 & 0.000 & 0.276 & 1.596 \\
\hline 96 Mo & A & 2 & 0.103 & 0.002 & 0.093 & 1.108 \\
\hline${ }^{97} \mathrm{Mo}$ & A & 1 & 0.330 & 0.000 & 0.330 & 1.000 \\
\hline${ }^{98} \mathrm{Mo}$ & $\mathrm{A}$ & 7 & 0.108 & 0.125 & 0.078 & 1.380 \\
\hline${ }^{100} \mathrm{Mo}$ & A & 4 & 0.099 & 0.034 & 0.095 & 1.043 \\
\hline${ }^{99} \mathrm{Tc}$ & A & 7 & 0.860 & 0.207 & 1.454 & 0.591 \\
\hline${ }^{96} \mathrm{Ru}$ & A & 1 & 0.312 & 0.000 & 0.162 & 1.931 \\
\hline${ }^{101} \mathrm{Ru}$ & A & 10 & 1.294 & 0.078 & 1.255 & 1.031 \\
\hline${ }^{102} \mathrm{Ru}$ & A & 11 & 0.214 & 0.051 & 0.133 & 1.603 \\
\hline${ }^{104} \mathrm{Ru}$ & $\mathrm{A}$ & 12 & 0.169 & 0.030 & 0.215 & 0.789 \\
\hline${ }^{103} \mathrm{Rh}$ & GS & 1 & 0.600 & 0.000 & 0.989 & 0.607 \\
\hline${ }^{103} \mathrm{Rh}$ & M1 & 1 & 0.045 & 0.000 & 0.070 & 0.640 \\
\hline${ }^{103} \mathrm{Rh}$ & A & 48 & 0.911 & 0.108 & 1.058 & 0.861 \\
\hline \multicolumn{5}{|c|}{ Average error: } & $\begin{array}{l}\text { error: } \\
\text { only): }\end{array}$ & $\begin{array}{l}33.2 \% \\
29.6 \%\end{array}$ \\
\hline
\end{tabular}

evaluate Maxwellian averaged capture cross sections for astrophysical applications (Bao et al. 2000). The Maxwellian-averaged neutron capture cross section is defined as the reaction rate $\langle\sigma v\rangle$ divided by the mean velocity $v_{T}=\sqrt{2 k T / \mu}$ at a given temperature $T$. Here, $\mu$ is the reduced mass. For particle fluences and temperatures typical to stellar nucleosynthesis, the velocity distribution of the neutrons is well described by a Maxwell-Boltzmann distribution. In this case, the Maxwellian-averaged cross section reduces to (Beer et al. 1992)

$$
\begin{aligned}
\frac{\langle\sigma v\rangle}{v_{T}} & =\frac{\int_{0}^{\infty} \sigma_{n \gamma} v \Phi(v) d v}{v_{T}} \\
& =\frac{2}{\sqrt{\pi}(k T)^{2}} \int_{0}^{\infty} \sigma_{n \gamma}(E) W(E, k T) d E
\end{aligned}
$$

where $W(E, k T)=E \exp (-E / k T)$ and $E$ is the center of mass energy.

Figure 11 compares our calculated Maxwellian-
Table 2: Comparison of our modeled Maxwellianaveraged (n, $\gamma$ ) cross sections (in millibarns) to rec-

\begin{tabular}{|c|c|c|c|}
\hline${ }^{{ }^{A} Z}$ & Recommended & Modeled & $\% \mathrm{E}$ \\
\hline${ }^{84} \mathrm{Sr}$ & $368 \pm 126$ & 404.5 & 9.9 \\
\hline${ }^{86} \mathrm{Sr}$ & $64 \pm 3$ & 69.7 & 8.9 \\
\hline${ }^{87} \mathrm{Sr}$ & $92 \pm 4$ & 176.2 & 91.6 \\
\hline${ }^{88} \mathrm{Sr}$ & $6.2 \pm 0.3$ & 12.8 & 106.6 \\
\hline${ }^{89} \mathrm{Sr}$ & $19 \pm 14$ & 27.0 & 42.3 \\
\hline${ }^{89} \mathrm{Y}$ & $19.0 \pm 0.6$ & 19.0 & 0.2 \\
\hline${ }^{90} \mathrm{Zr}$ & $21 \pm 2$ & 26.2 & 24.9 \\
\hline${ }^{91} \mathrm{Zr}$ & $60 \pm 8$ & 63.1 & 5.1 \\
\hline${ }^{92} \mathrm{Zr}$ & $33 \pm 4$ & 41.9 & 27.1 \\
\hline${ }^{93} \mathrm{Zr}$ & $95 \pm 10$ & 191.2 & 101.2 \\
\hline${ }^{94} \mathrm{Zr}$ & $26 \pm 1$ & 25.8 & 0.6 \\
\hline${ }^{95} \mathrm{Zr}$ & $79 \pm 12$ & 116.7 & 47.8 \\
\hline${ }^{96} \mathrm{Zr}$ & $10.7 \pm 0.5$ & 18.1 & 69.2 \\
\hline${ }^{93} \mathrm{Nb}$ & $266 \pm 5$ & 305.7 & 14.9 \\
\hline${ }^{94} \mathrm{Nb}$ & $482 \pm 92$ & 609.5 & 26.4 \\
\hline${ }^{95} \mathrm{Nb}$ & $310 \pm 65$ & 379.9 & 22.6 \\
\hline${ }^{92} \mathrm{Mo}$ & $70 \pm 10$ & 64.5 & 7.9 \\
\hline${ }^{94} \mathrm{Mo}$ & $102 \pm 20$ & 96.7 & 5.2 \\
\hline${ }^{95} \mathrm{Mo}$ & $292 \pm 12$ & 268.1 & 8.2 \\
\hline${ }^{96} \mathrm{Mo}$ & $112 \pm 8$ & 89.8 & 19.8 \\
\hline${ }^{97} \mathrm{Mo}$ & $339 \pm 14$ & 310.9 & 8.3 \\
\hline${ }^{98} \mathrm{Mo}$ & $99 \pm 7$ & 79.6 & 19.6 \\
\hline${ }^{99} \mathrm{Mo}$ & $240 \pm 40$ & 231.8 & 3.4 \\
\hline${ }^{100} \mathrm{Mo}$ & $108 \pm 14$ & 98.1 & 9.2 \\
\hline${ }^{99} \mathrm{Tc}$ & $781 \pm 50$ & 1377.8 & 76.4 \\
\hline${ }^{96} \mathrm{Ru}$ & $238 \pm 60$ & 161.6 & 32.1 \\
\hline${ }^{98} \mathrm{Ru}$ & $173 \pm 36$ & 184.3 & 6.5 \\
\hline${ }^{99} \mathrm{Ru}$ & $631 \pm 99$ & 997.9 & 58.1 \\
\hline${ }^{100} \mathrm{Ru}$ & $206 \pm 13$ & 177.2 & 14.0 \\
\hline${ }^{101} \mathrm{Ru}$ & $996 \pm 40$ & 1186.6 & 19.1 \\
\hline${ }^{102} \mathrm{Ru}$ & $186 \pm 11$ & 136.7 & 26.5 \\
\hline${ }^{103} \mathrm{Ru}$ & $343 \pm 52$ & 502.9 & 46.6 \\
\hline${ }^{104} \mathrm{Ru}$ & $161 \pm 10$ & 213.0 & 32.3 \\
\hline${ }^{103} \mathrm{Rh}$ & $811 \pm 14$ & 972.3 & 19.9 \\
\hline \multicolumn{3}{|c|}{ Average percent error: } & $29.8 \%$ \\
\hline
\end{tabular}
ommended values at $30 \mathrm{keV}$

averaged capture cross sections for select targets to their evaluated counterparts (Bao et al. 2000). The error bars on all points are identical and represent the measured error for a given cross section at $30 \mathrm{keV}$. We used spline interpolation to determine the value of the $(n, \gamma)$ cross section between points on the energy grid. For energies below our lowest grid energy, we assume an $(\mathrm{n}, \gamma)$ cross section with an $E_{l a b}^{-1 / 2}$ dependence. For energies greater than our highest grid energy, we take the cross section to be zero. 

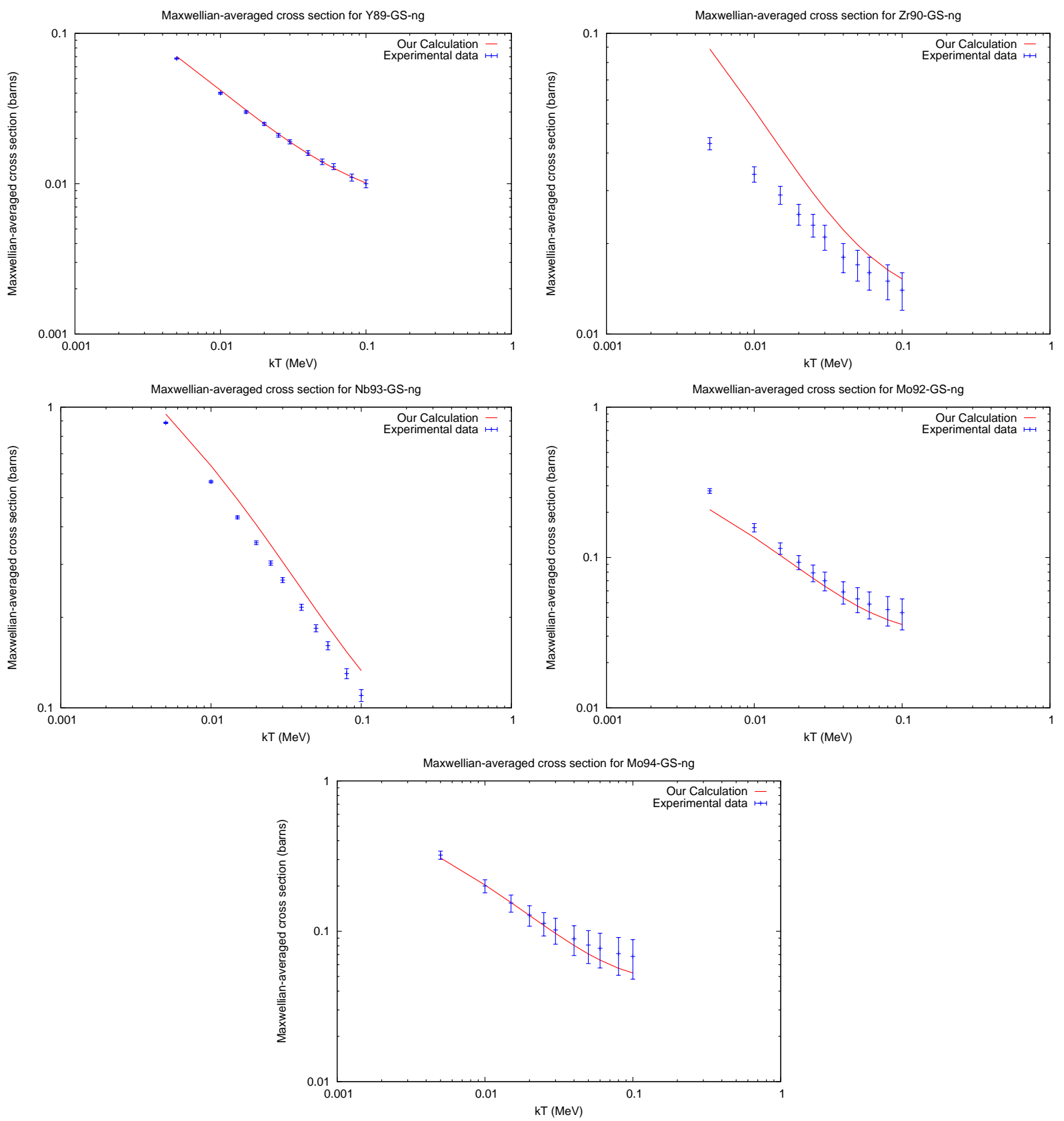

Fig. 11. - Calculated vs. recommended Maxwellian-averaged capture cross sections on select stable isotopes in the region of interest. The data is taken from (Bao et al. 2000). The solid line represent our modeled cross section. The data points are the recommended values, with the error bars for each energy identical to the quoted error at $30 \mathrm{keV}$.

Overall our calculated Maxwellian averaged cross sections agree with those of (Bao et al. 2000). Our result for ${ }^{90} \mathrm{Zr}$ is a bit high, consistent with Figure 10. Additional comparisons found in Appendix C.2 exhibit similar agreement.

It is also useful to establish a quantitative measure of how well our model calculations can replicate the recommended $30 \mathrm{keV}$ Maxwellianaveraged cross sections. We define the average percent error as

$$
\% E=\frac{1}{N} \sum_{i=1}^{N} \frac{\left|\frac{\left\langle\sigma_{i}^{m o d} v\right\rangle}{v_{T}}-\frac{\left\langle\sigma_{i}^{r e c} v\right\rangle}{v_{T}}\right|}{\frac{\left\langle\sigma_{i}^{r e c} v\right\rangle}{v_{T}}} \times 100 \%
$$


The errors for each of our modeled $30 \mathrm{keV}$ Maxwellian-averaged cross sections, relative to the recommended values, are listed in Table 2 . Listed in the table are the target, recommended cross section (with error, in millibarns), our modeled value, and the percent error. On average, our modeled Maxwellian averaged capture cross sections are within $30 \%$ of the recommended values, consistent with our findings in Table 1.

\subsubsection{Comparison to experimental $(n, 2 n)$ cross sections}

Our calculated $(\mathrm{n}, 2 \mathrm{n})$ cross sections for select targets are presented in Figure 12. We begin with ${ }^{88} \mathrm{Y}(\mathrm{n}, 2 \mathrm{n}){ }^{87} \mathrm{Y}$, a rare case where an $(\mathrm{n}, 2 \mathrm{n})$ cross section has been measured for a radioactive target. The amount of data is understandably limited, consisting of two measurements near 14 and $15 \mathrm{MeV}$. Our calculation is within the error bar for the point at $14.8 \mathrm{MeV}$, and only slightly below the error bar for the point at $14.2 \mathrm{MeV}$.

As one would expect, the ${ }^{89} \mathrm{Y}(\mathrm{n}, 2 \mathrm{n})^{88} \mathrm{Y}$ cross section has been measured in many different experiments. For the most part, the various data sets are in good agreement. We note that a few of the data sets, indicated by the open black data points at $15 \mathrm{MeV}$, are not identified as going to the final state (i.e. the data file does not specify whether the measured cross section leads to the ground state, isomer, etc...). It is likely that these measurements are cross sections leading to the first or second isomer. Overall, our calculation is in good agreement with the data. An improvement could be made by adjusting the pre-equilibrium parameters, as will be discussed in section 4.2.1.

Our calculation also exhibits good agreement against measured data for the ${ }^{88} \mathrm{Zr}(\mathrm{n}, 2 \mathrm{n})^{87} \mathrm{Zr}$ reaction. Again, this cross section has been measured numerous times, and the various data sets are generally consistent with each other. We can also achieve slightly better agreement with this cross section by modifying the pre-equilibrium parameters, but only in a manner which would reduce the agreement with ${ }^{89} Y(n, 2 n)^{88} Y$. See section 4.2 .1 for details. We note that ${ }^{88} \mathrm{Zr}(\mathrm{n}, 2 \mathrm{n})^{87} \mathrm{Zr}$ has been measured at $14.8 \mathrm{MeV}$, and our calculation is in near perfect agreement with this measurement (see Appendix C.3).

The ${ }^{93} \mathrm{Nb}(\mathrm{n}, 2 \mathrm{n})^{92} \mathrm{Nb}$ calculation is in good agreement with measured activation cross section data. The relative amounts of cross section leading to the first and second isomers (blue and green lines, respectively), however, does not appear to correspond with the measurements. The source of this disagreement lies primarily in our assignment of isomers. We treat as isomers any state with a lifetime greater than $1 \mu s$. The lifetime of the first isomer is quite long (10 days), while that of the second isomer is only $5.9 \mu \mathrm{s}$. This second excited state, which decays via internal transition to the first isomer, would not normally be considered an isomer itself, and therefore measurements would include the population of the second excited state into the cross section leading to the first isomer. One will note that the sum of our first and second isomer cross sections would lie in near perfect agreement with the measured first isomer cross section.

Our calculation reproduces the measured ${ }^{92} \mathrm{Mo}(\mathrm{n}, 2 \mathrm{n}){ }^{91} \mathrm{Mo}$ activation cross section up to about $16 \mathrm{MeV}$. The agreement with the cross section leading to the isomer is in good agreement for the entire energy range. There is a considerable amount of spread in the data for the cross section leading to the ground state, as well as the cross section leading to the isomer below $15 \mathrm{MeV}$.

The data for ${ }^{94} \mathrm{Mo}(\mathrm{n}, 2 \mathrm{n}){ }^{93} \mathrm{Mo}$ is quite limited. Our cross section leading to the isomer is in excellent agreement with the data. However, our calculation is higher than the lone activation cross section data point by more than a factor of two.

Appendix C.3 presents additional comparisons between our modeled (n,2n) cross sections and experiment. We note that there is a significant disagreement between the measured activation data sets for ${ }^{84} \mathrm{Sr}(\mathrm{n}, 2 \mathrm{n}){ }^{83} \mathrm{Sr}$, and that the lowest data set may be a misidentified cross section leading to the isomer. Also, we note for ${ }^{91} \mathrm{Zr}(\mathrm{n}, 2 \mathrm{n}){ }^{90} \mathrm{Zr}$ that the measured cross section leading to the first isomer is in excellent agreement with our cross section leading to the third isomer. This is due to our considering the first two excited states of ${ }^{90} \mathrm{Zr}$, whose lifetimes are $61.3 \mathrm{~ns}$ and 88.4 fs, respectively, to be isomers, consistent with what was done in the previous RADCHEM data sets. Our third isomer (lifetime $809 \mathrm{~ms}$ ) corresponds to the state identified as the first isomer in the experiments. See section 3.1.2.

As a further means of evaluating our $(n, 2 n)$ cross sections, we present in Table 3 a quantified comparison of our results to the experimental data shown in Figures 12 and 27. We include all data with incident energies of $14.1 \pm 0.1 \mathrm{MeV}$. The format of the table is identical to Table 1 .

The average deviation of our cross sections from the weighted means for the $(n, 2 n)$ reac- 

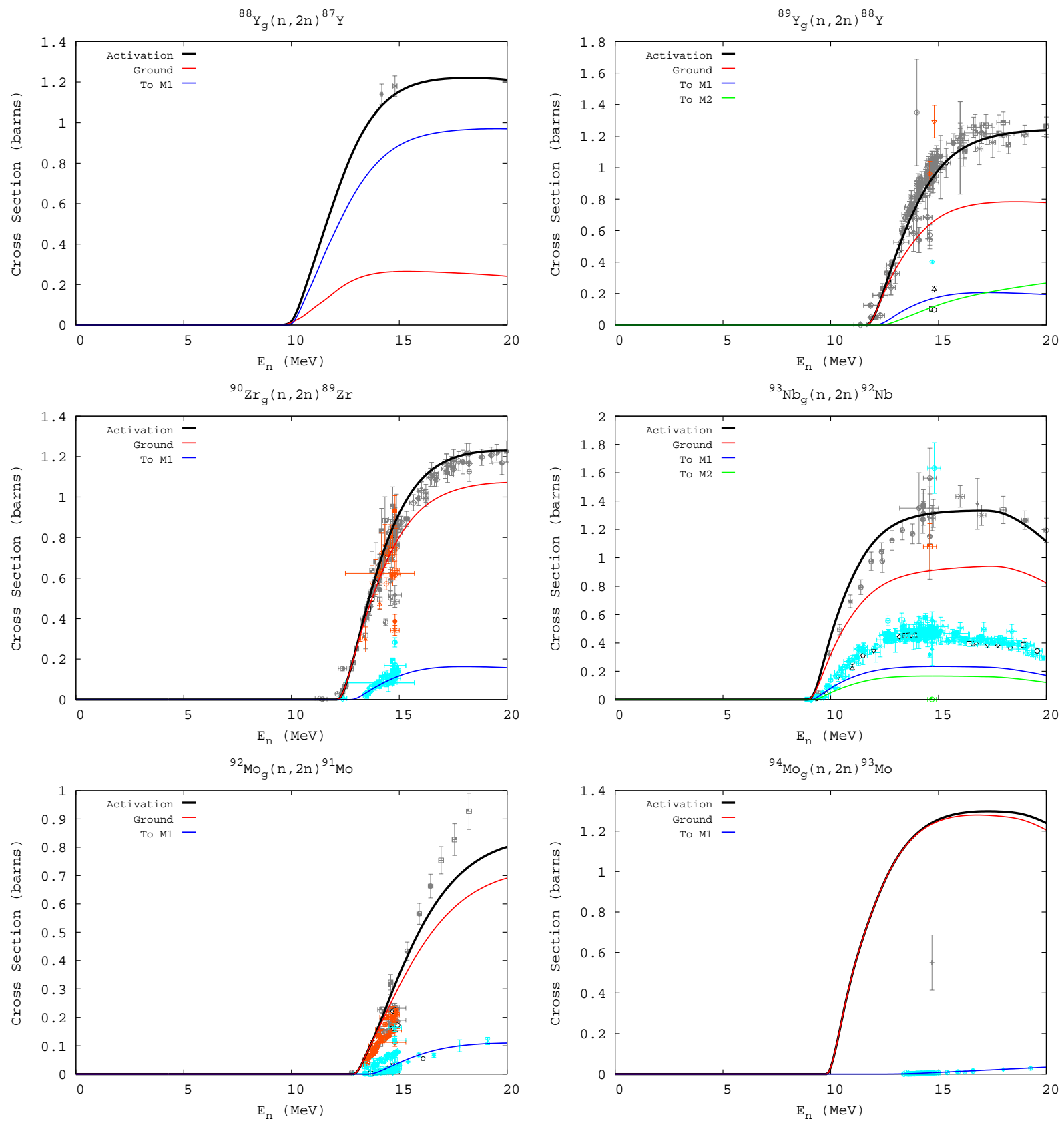

Fig. 12.- Calculated vs. measured $(\mathrm{n}, 2 \mathrm{n})$ cross sections on select stable isotopes in the region of interest. The data is taken from (EXFOR 2006). The black, red, and blue solid lines represent our modeled cross sections (total, leading to the ground state, and leading to the first isomer, respectively). The Gery, orange, and light blue data points are measured cross section data (total, ground state, and first isomer).

tions is $16.6 \%$ when only activation measurements are considered. For three cases (namely ${ }^{84} \mathrm{Sr}(\mathrm{n}, 2 \mathrm{n}){ }^{83} \mathrm{Sr},{ }^{92} \mathrm{Mo}(\mathrm{n}, 2 \mathrm{n}){ }^{91} \mathrm{Mo},{ }^{96} \mathrm{Ru}(\mathrm{n}, 2 \mathrm{n}){ }^{95} \mathrm{Ru}$, and $\left.{ }^{103} \operatorname{Rh}(\mathrm{n}, 2 \mathrm{n}){ }^{102} \mathrm{Rh}\right)$ there are several activation data sets that are not in agreement with each other. In each of these cases, our calculation lies close to at least one of the data sets. Excluding these three reactions, the average deviation for the $(\mathrm{n}, 2 \mathrm{n})$ activation cross sections is $6.4 \%$.

Larger deviations occur for the cross sections to specific final states (ground state, isomers). When these are included in the average, the deviation is closer to $20 \%$ ( $12.5 \%$ when the three reactions noted above and ${ }^{103} \mathrm{Rh}(\mathrm{n}, 2 \mathrm{n})^{102} \mathrm{Rh}_{m 1}$, which ex- 
Table 3: Comparison of our modeled (n,2n) cross sections to experimental data at $14.1 \pm 0.1 \mathrm{MeV}$

\begin{tabular}{|c|c|c|c|c|c|c|}
\hline${ }^{A} Z$ & Res. & $\mathrm{N}$ & $\bar{\sigma}$ & Dev. & $\sigma_{\text {mod }}$ & $\bar{\sigma} / \sigma_{m o}$ \\
\hline${ }^{84} \mathrm{Sr}$ & A & 4 & 0.526 & 0.349 & 0.558 & 0.942 \\
\hline${ }^{86} \mathrm{Sr}$ & GS & 1 & .547 & 0.000 & 0.639 & 0.856 \\
\hline${ }^{86} \mathrm{Sr}$ & M1 & 3 & .257 & 0.043 & 0.184 & 1.397 \\
\hline${ }^{86} \mathrm{Sr}$ & A & 2 & .731 & 0.034 & 0.823 & 0.888 \\
\hline${ }^{88} \mathrm{Sr}$ & M1 & 5 & .225 & 0.029 & 0.209 & 1.077 \\
\hline${ }^{88} \mathrm{Y}$ & A & 1 & 1.140 & 0.000 & 1.073 & 1.062 \\
\hline${ }^{89} \mathrm{Y}$ & A & 22 & .853 & 0.147 & 0.791 & 1.078 \\
\hline${ }^{90} \mathrm{Zr}$ & GS & 4 & .520 & 0.120 & & .862 \\
\hline${ }^{90} \mathrm{Zr}$ & M1 & 11 & .078 & 0.0 & & .062 \\
\hline${ }^{90} \mathrm{Zr}$ & A & 21 & 0.618 & 0.0 & 0.676 & 915 \\
\hline${ }^{96} \mathrm{Zr}$ & A & 5 & 1.544 & 0.0 & 1.536 & 1.005 \\
\hline${ }^{93} \mathrm{Nb}$ & M1 & 37 & .459 & & & 1.973 \\
\hline${ }^{93} \mathrm{Nb}$ & A & 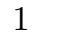 & 1.350 & 0.000 & 1.2 & 1.041 \\
\hline${ }^{92} \mathrm{Mo}$ & GS & 8 & .123 & 0.032 & 0.170 & 0.726 \\
\hline${ }^{92} \mathrm{Mo}$ & M1 & 14 & .008 & 0.019 & 0.010 & 0.848 \\
\hline${ }^{92} \mathrm{Mo}$ & A & 8 & 129 & 0.044 & 0.180 & 0.716 \\
\hline${ }^{94} \mathrm{Mo}$ & M1 & 5 & 003 & 0.000 & 0.004 & 0.779 \\
\hline${ }^{100} \mathrm{Mo}$ & $\mathrm{A}$ & 9 & 1.472 & 0.162 & 1.537 & 0.958 \\
\hline${ }^{96} \mathrm{Ru}$ & A & 4 & 0.576 & 0.032 & 0.870 & 0.661 \\
\hline${ }^{98} \mathrm{Ru}$ & A & 2 & 1.093 & 0.102 & 1.206 & 0.906 \\
\hline${ }^{4} \mathrm{Ru}$ & A & 3 & 1.607 & 0.531 & 1.556 & 1.033 \\
\hline${ }^{3} \mathrm{Rh}$ & GS & 5 & 0.677 & 0.111 & 0.608 & 1.113 \\
\hline${ }^{103} \mathrm{Rh}$ & M1 & 5 & 0.494 & 0.093 & 0.762 & 0.648 \\
\hline${ }^{103} \mathrm{Rh}$ & A & 4 & 0.846 & 0.113 & 1.369 & 0.618 \\
\hline \multicolumn{6}{|c|}{ Average error: } & $\begin{array}{l}20.3 \% \\
16.6 \% \\
\end{array}$ \\
\hline
\end{tabular}

hibits a similar disparity in the measured data, and ${ }^{93} \mathrm{Nb}(\mathrm{n}, 2 \mathrm{n}){ }^{92} \mathrm{Nb}_{m 1}$, as discussed earlier, are excluded).

\subsubsection{Comparison to experimental $(n, p)$ cross sections}

For most stable isotopes, the neutron capture and $(\mathrm{n}, 2 \mathrm{n})$ reactions are the dominant neutron induced reaction channels at low and high incident energies, respectively. However, as one moves to the proton rich side of stability, proton separation energies become small, and reaction channels involving charged particles in the exit channel, specifically $(n, p)$ and $(n, n p)$ can become dominant.

In Figure 13 we present our modeled cross sections for select target isotopes compared to measurement. For ${ }^{89} \mathrm{Y}(\mathrm{n}, \mathrm{p}){ }^{89} \mathrm{Sr}$ our calculation is in good agreement with the data between threshold and $\sim 10 \mathrm{MeV}$. At $14 \mathrm{MeV}$ incident energy, our calculation lies roughly $50 \%$ higher than the bulk of the experimental data. This behavior is a common feature in our $(n, p)$ cross sections, and is likely due to the simple treatment of pre-equilibrium. Fortunately, the $(\mathrm{n}, \mathrm{p})$ cross section is rarely dominant above $\sim 12 \mathrm{MeV}$ (see Appendix D), so that errors in our calculation at and above $14 \mathrm{MeV}$ will likely have insignificant impact in network calculations.

Our results for the other three targets in Figure 13 are in better agreement with experiment up to $\sim 15 \mathrm{MeV}$ incident energy. We note that for the ${ }^{92} \mathrm{Mo}(\mathrm{n}, \mathrm{p}){ }^{92} \mathrm{Nb}$ reaction the same situation arises that we saw for ${ }^{93} \mathrm{Nb}(\mathrm{n}, 2 \mathrm{n}){ }^{92} \mathrm{Nb}$ (Section 4.1.3) where the sum of our cross sections leading to the first and second isomer in ${ }^{92} \mathrm{Nb}$ sum roughly to the measured cross section to the first isomer. We remind the reader that this is due to our considering the second excited state (lifetime $5.9 \mu \mathrm{s}$, decaying to the first isomer) as an isomer, where it would not be considered an isomer in the experiments.

Additional comparisons of our $(\mathrm{n}, \mathrm{p})$ cross sections to experiment can be found in Appendix C.6. The agreement between the calculation and measurement in comparable to that presented in Figure 13.

Due to the spread in the data in measured $(\mathrm{n}, \mathrm{p})$ cross sections and the presence of large error bars, a quantitative analysis of our calculations similar to that found in Tables 1 and 3 is difficult. For example, our calculation for ${ }^{100} \mathrm{Ru}(\mathrm{n}, \mathrm{p})^{100} \mathrm{Tc}$ is actually in good agreement with the data, but averaging the measured cross sections at $14.8 \mathrm{MeV}$ would suggest our cross section is high by nearly a factor of two. There are also several instances where the activation cross section data is not consistent with the sum of the measured cross sections leading to the ground state and isomers.

\subsubsection{Comparison to experimental $(n, n p)$ cross sections}

On the proton rich side of stability, the $(\mathrm{n}, \mathrm{np})$ reaction can be larger than the $(\mathrm{n}, 2 \mathrm{n})$ reaction near $14 \mathrm{MeV}$ incident energy (see Appendix D). Unfortunately, experimental data for this reaction is sparse in this region. Available measurements generally consist of only a few data points near threshold, with cross sections on the order of $10 \mathrm{mb}$ or less (see Figure 31 of Appendix C.7). Only two $(\mathrm{n}, \mathrm{np})$ reactions in this region have measured data in an energy range where the cross section is greater than a few hundred millibarns. These are shown in Figure 14. For ${ }^{88} \mathrm{Zr}(\mathrm{n}, \mathrm{np}){ }^{87} \mathrm{Y}$, the measured activation cross section is $253 \pm 25 \mathrm{mb}$ at $14.8 \pm 0.1 \mathrm{MeV}$ incident en- 

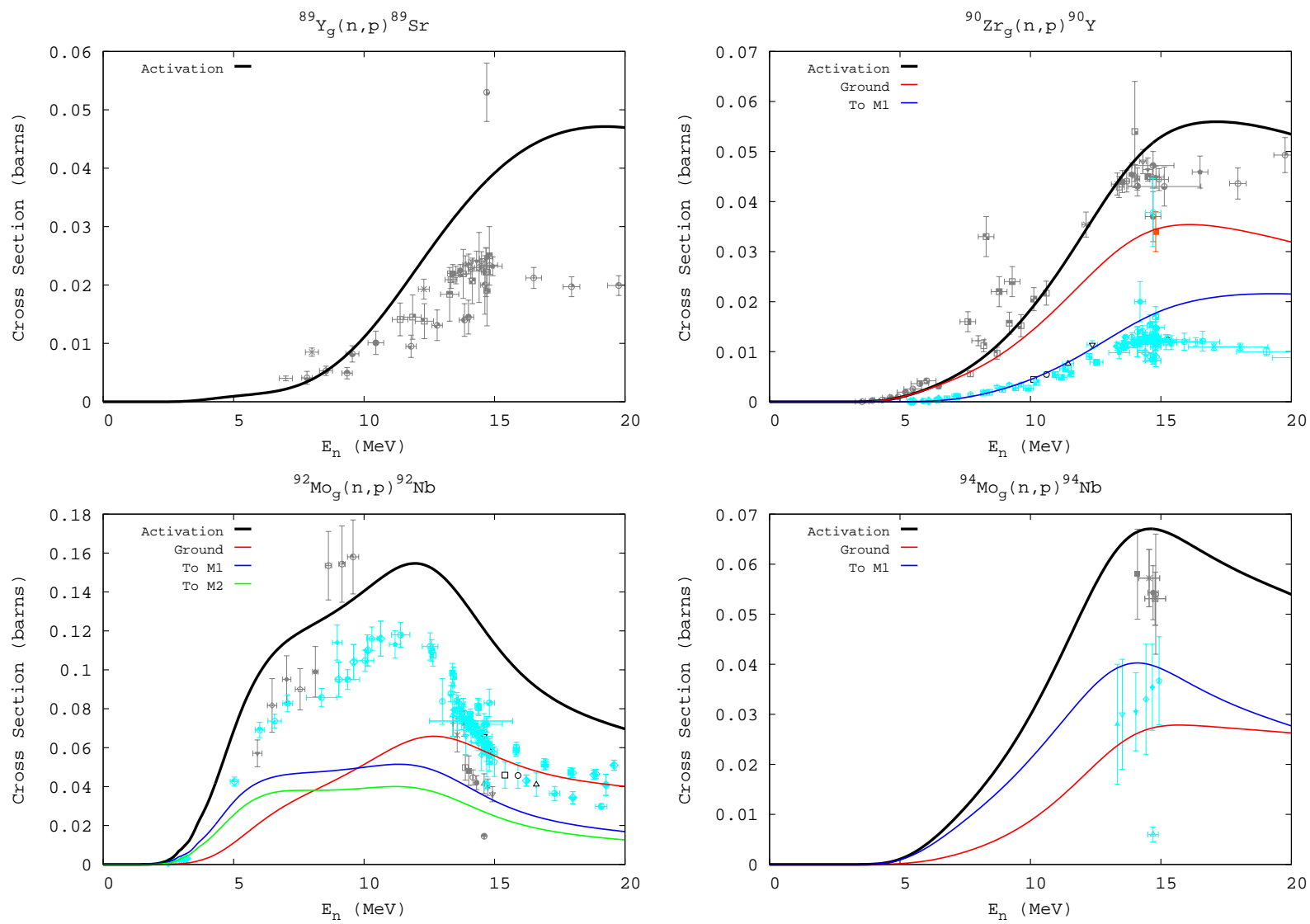

Fig. 13.- Calculated vs. measured (n,p) cross sections on select stable isotopes in the region of interest. The data is taken from (EXFOR 2006). The black, red, and blue solid lines represent our modeled cross sections (total, leading to the ground state, and leading to the first isomer, respectively). The Gery, orange, and light blue data points are measured cross section data (total, ground state, and first isomer).
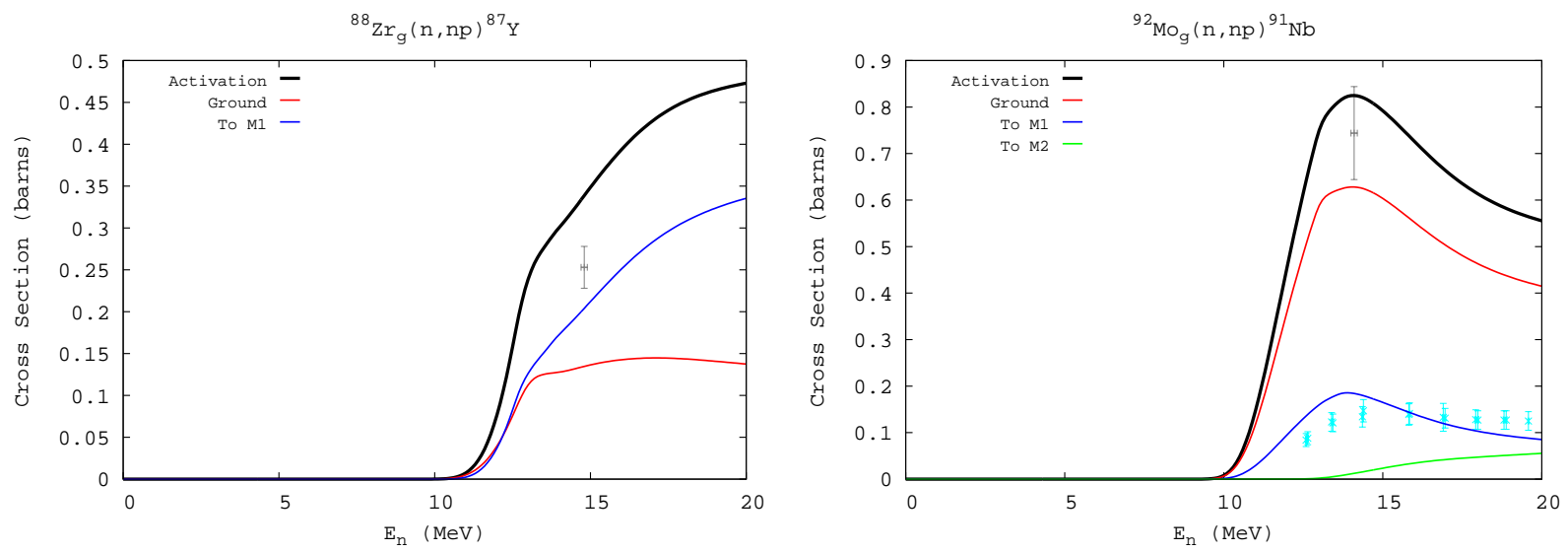

Fig. 14.- Calculated vs. measured (n,np) cross sections on select stable isotopes in the region of interest. The data is taken from (EXFOR 2006). The black, red, and blue solid lines represent our modeled cross sections (total, leading to the ground state, and leading to the first isomer, respectively). The Gery, orange, and light blue data points are measured cross section data (total, ground state, and first isomer).

ergy (Prestwood et al. 1984). Our calculation for this reaction yields a cross section of $338.7 \mathrm{mb}$ at
14.8 $\mathrm{MeV}$, which is high compared to the measurement by $34 \%$. For ${ }^{92} \mathrm{Mo}(\mathrm{n}, \mathrm{np})^{91} \mathrm{Nb}$, our calculated 

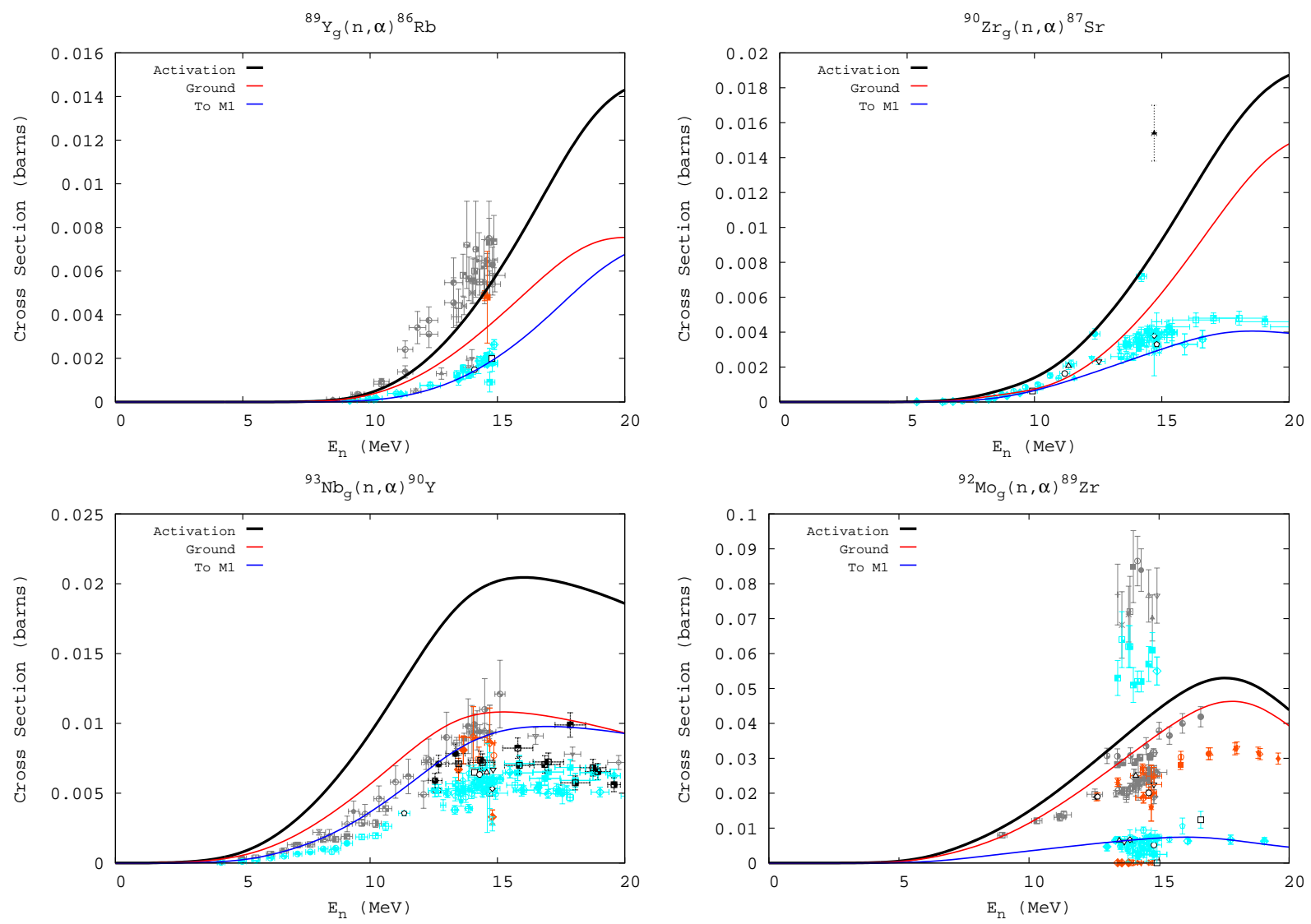

Fig. 15.- Calculated vs. measured $(\mathrm{n}, \alpha)$ cross sections on select stable isotopes in the region of interest. The data is taken from (EXFOR 2006). The black, red, and blue solid lines represent our modeled cross sections (total, leading to the ground state, and leading to the first isomer, respectively). The Gery, orange, and light blue data points are measured cross section data (total, ground state, and first isomer).

activation cross section is within the errors of the measured data, and the agreement with the measured cross section leading to the isomer of ${ }^{91} \mathrm{Nb}$ is good.

The scarcity of measured (n,np) data makes it difficult to estimate the accuracy at which we can predict $(\mathrm{n}, \mathrm{np})$ cross sections.

\subsubsection{Comparison to experimental $(n, \alpha)$ cross sections}

The $(\mathrm{n}, \alpha)$ channel is generally small compared to other open channels. However, due to the prominence of $(\mathrm{n}, \alpha)$ cross sections in the Mo1278 detector set, we make special mention of them here.

The availability and quality of $(\mathrm{n}, \alpha)$ data in this region is comparable to what is available for $(n, p)$. There are occasions where the spread in the data is quite large, and many of the data points have rather large error bars. Again, this precludes us from making a meaningful quantitative analysis of our $(\mathrm{n}, \alpha)$ calculations. However, we present in Figure 15 a sample of our results compared to data for a few select targets. Additional comparisons can be found in Appendix C.8.

For ${ }^{89} \mathrm{Y}(\mathrm{n}, \alpha)^{86} \mathrm{Rb}$, our calculation is in good agreement with experiment. For ${ }^{90} \mathrm{Zr}(\mathrm{n}, \alpha){ }^{87} \mathrm{Sr}$, only data for cross section leading to the isomer is available (the other data points did not unambiguously designate a final state). Our cross section is in reasonable agreement with the bulk of the measured data. Our modeled cross section for ${ }^{93} \mathrm{Nb}(\mathrm{n}, \alpha){ }^{90} \mathrm{Y}$ is high, though we note that the activation cross section data is in conflict with the bulk of the data to the ground state and isomer, i.e. the sum of the ground state and isomer cross sections would yield an activation cross section somewhat higher than what has been measured. We note particularly that our cross section to the ground state is in reasonably fair agreement with 
experiment, being high be perhaps $\sim 20 \%$. Our cross section to the isomer of ${ }^{90} \mathrm{Y}$ appears to be high by about $60 \%$. Our activation cross section would be $\sim 40-50 \%$ higher than the sum of the measured ground state and isomer cross sections.

The experimental data for ${ }^{92} \mathrm{Mo}(\mathrm{n}, \alpha)^{89} \mathrm{Zr}$ is rather disparate. There are two distinct groups of measurements, each involving several experiments, for the activation cross section. The same is true for the cross sections leading to the ground state and first isomer. Our calculated cross section leading to the isomer is in very good agreement with the lower set of isomer data, while our ground state cross section (and consequently our activation cross section) appear to be high by $\sim 20$ $25 \%$ at $14 \mathrm{MeV}$.

Similar agreement will be found for the other comparisons in Appendix C.8. For the most part, our calculations do quite well, with a few notable exceptions, including ${ }^{99} \mathrm{Tc}(\mathrm{n}, \alpha){ }^{96} \mathrm{Nb}$ and ${ }^{103} \operatorname{Rh}(\mathrm{n}, \alpha)^{100} \mathrm{Tc}$, for which we are high by a factor of $\sim 2$.

\subsubsection{Comparison to other experimental neutron induced cross sections}

We have also made comparisons for other neutron induced reaction channels, described here briefly.

For the two $(n, 3 n)$ reactions for which data is available (Appendix C.4) our calculations fare quite well. However, the thresholds for these reactions are well above $14 \mathrm{MeV}$.

Our calculations agree nicely with the measured (n,n') data (Appendix C.5). We remind the reader of the issue regarding the isomers in ${ }^{90} \mathrm{Zr}$, as explained in section 3.1.2, which is why our (n,n') cross section for that target leaving the nucleus in the third isomer compares favorably to the data leading to the first isomer.

The $(\mathrm{n}, \mathrm{n} \alpha)$ channel is always very small compared to the dominant channel, and the data is limited (Appendix C.9). A similar statement can be made of the $(n, d)$ cross sections (Appendix C.10).

\subsubsection{Comparison to experimental $(p, n)$ cross sections}

Our calculations for $(\mathrm{p}, \mathrm{n})$ cross sections are compared to experimental data for four select targets in Figure 16. The black data on the plot for ${ }^{89} \mathrm{Y}(\mathrm{p}, \mathrm{n})^{89} \mathrm{Zr}$ represents measurements from (Mustafa et al. 1988), used in the Yt0488 detec- tor set. Our calculation runs higher than the measurement by roughly $15 \%$, with the overall shape in good agreement. The amounts going to the ground state and isomer are somewhat more uncertain. Our ground state cross section is in good agreement with one data set (solid red squares), but is significantly higher than the other larger set (red open squares). Keep in mind that the measured cross section will be used for radiochemical analysis in lieu of this calculation.

The agreement between our calculated activation cross section and experiment is satisfactory for ${ }^{90} \mathrm{Zr}(\mathrm{p}, \mathrm{n})^{90} \mathrm{Nb}$. The amounts going to the various final states of ${ }^{90} \mathrm{Nb}$ are more in question. Certainly the isomer data set below the reaction threshold is not to be believed. The experimental isomer data is most likely associated with the second excited state (lifetime 18.8 seconds), which is denoted as the second isomer in our modeling effort. The first excited state (first isomer in our modeling, lifetime $63 \mu \mathrm{s}$ ) decays to the ground state, and the eighth excited state (third isomer in our calculation, lifetime $6.19 \mathrm{~ms}$ ) decays to the second excited state (second isomer in our calculation). So, in reality the ground state data (red data points) should be compared to the sum of our ground state and first isomer calculations (red and blue lines). The comparison would be fairly good, though the data is limited. The experimental isomer data (light blue points) should be compared to the sum of our second and third isomer cross sections (green and magenta lines). It appears that our calculation would overestimate the measured isomer cross section.

Our calculated ${ }^{93} \mathrm{Nb}(\mathrm{p}, \mathrm{n}){ }^{93}$ Mo activation cross section is in excellent agreement with experiment. The cross section leading to the isomer in ${ }^{93} \mathrm{Mo}$ also does quite well up to $15 \mathrm{MeV}$ incident energy. For ${ }^{94} \mathrm{Mo}(\mathrm{p}, \mathrm{n}){ }^{94} \mathrm{Tc}$, our cross section leading to the ground state is in fair agreement with the data, though it appears out isomer and activation cross sections may be a bit high.

Additional comparisons to measured $(\mathrm{p}, \mathrm{n})$ cross sections can be found in Appendix C.11. Overall, our calculations generally do well in reproducing the experimental data, typically to within $\sim 20 \%$. We note that for ${ }^{100} \mathrm{Ru}(\mathrm{p}, \mathrm{n}){ }^{100} \mathrm{Rh}$ our discrete level scheme for ${ }^{100} \mathrm{Ru}$ did not encompass the isomer, which prevents us from calculating isomer and ground state cross sections. We also note (yet again) that the sum of the first and second isomer cross sections of ${ }^{92} \mathrm{Zr}(\mathrm{p}, \mathrm{n})^{92} \mathrm{Nb}$ should be summed for comparison to the measured isomer data. 

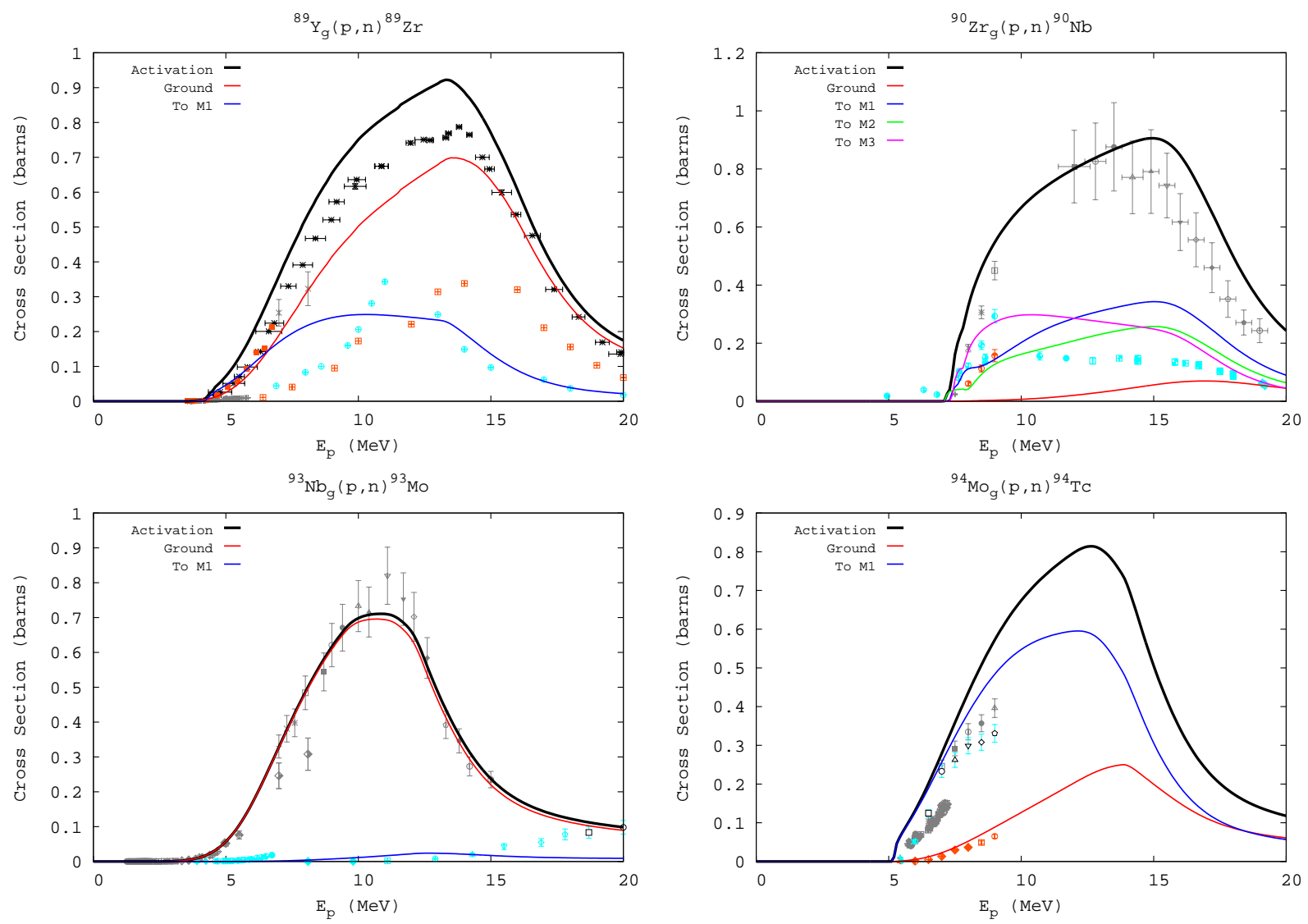

Fig. 16.- Calculated vs. measured $(\mathrm{p}, \mathrm{n})$ cross sections on select stable isotopes in the region of interest. The data is taken from (EXFOR 2006). The black, red, and blue solid lines represent our modeled cross sections (total, leading to the ground state, and leading to the first isomer, respectively). The Gery, orange, and light blue data points are measured cross section data (total, ground state, and first isomer).

\subsubsection{Comparison to other experimental charged particle cross sections}

In general, the available data for other charged particle reactions, including (p,2n), (p,pn), (p,p'), $(\mathrm{d}, \mathrm{n}),(\mathrm{d}, 2 \mathrm{n})$, and $(\mathrm{d}, 3 \mathrm{n})$, is sparse. We provide comparisons between our calculations and measurement for these reactions in Appendices C.12C.19. Our calculations for the proton induced reactions are typically in good agreement with the data, keeping in mind that the first and second isomer cross sections for ${ }^{93} \mathrm{Nb}(\mathrm{p}, \mathrm{np}){ }^{92} \mathrm{Nb}$ should be summed before comparing to the data (see Section 4.1.3). We also note that our calculation for ${ }^{89} \mathrm{Y}\left(\mathrm{p}, \mathrm{p}^{\prime}\right)^{89} \mathrm{Y}$ is several orders of magnitude greater than the measurements. Whether this is a result of errors in the modeling or in reporting the data has yet to be determined.

We also may make a few general statements regarding our modeled deuteron induced cross sections. By far, the greatest difficulty in modeling these cross sections accurately lies in the breakup calculations. These calculations are in turn highly dependent on the choice of optical potential employed. Our calculated $(\mathrm{d}, \mathrm{n})$ cross sections are generally in good agreement with the data near threshold, but then tend to either over- or underestimate the peak significantly. We note that the peak of this cross section occurs usually between $5-10 \mathrm{MeV}$ of incident energy, which is also where the deuteron breakup fraction (the fraction of reaction cross section used in deuteron breakup) is typically the largest. We find a somewhat better agreement between our modeled $(\mathrm{d}, 2 \mathrm{n})$ cross sections and the data, usually being within $20 \%$. The available $(\mathrm{d}, 3 \mathrm{n})$ data is limited to a single point at $19 \mathrm{MeV}$ incident energy for the ${ }^{89} \mathrm{Y}$ target.

We make note that the charged particle cross sections of greatest import in radiochemical analysis, i.e. those leading directly from the loaded detector elements to the measured radioactive 

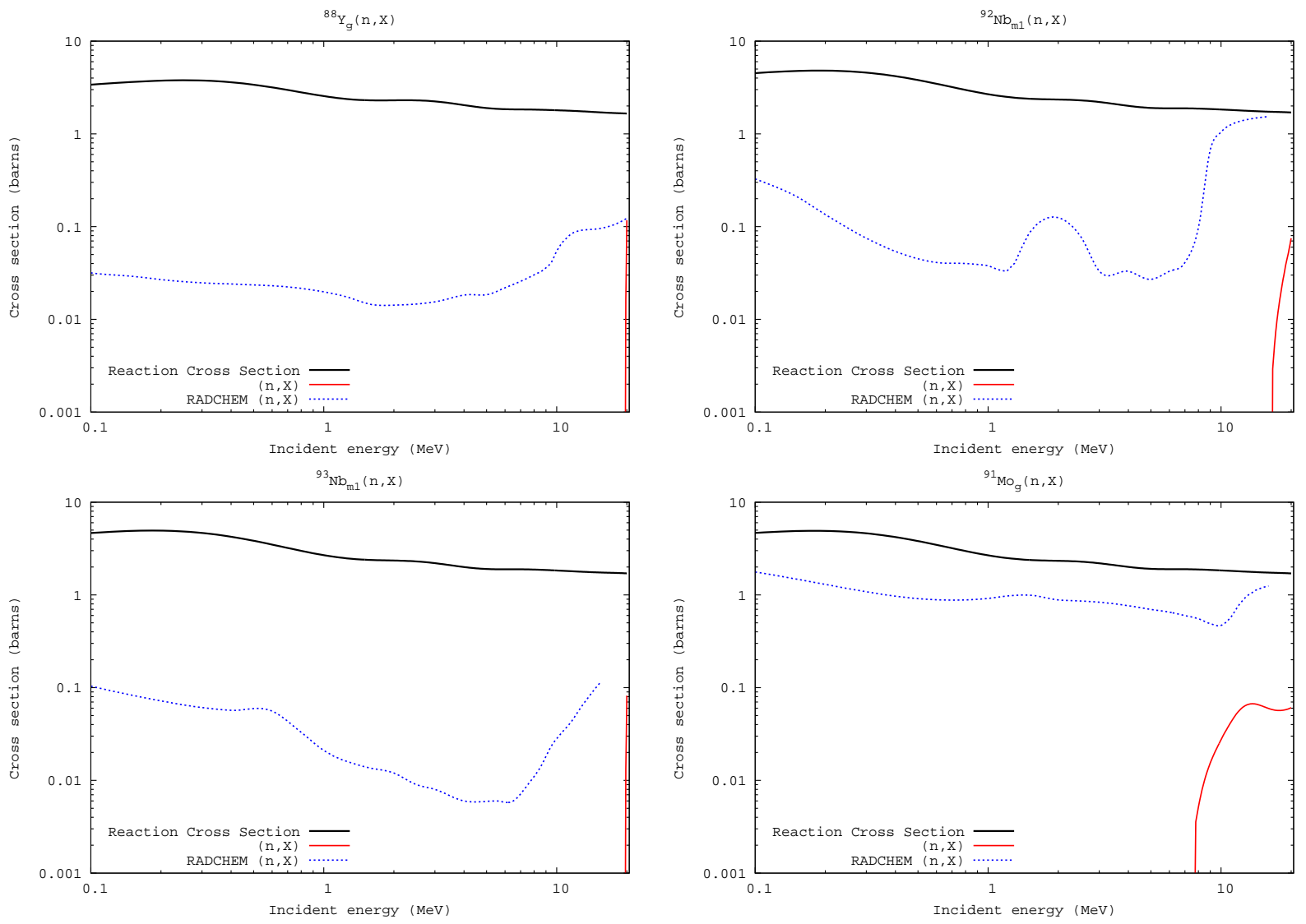

Fig. 17.- Calculated $(\mathrm{n}, \mathrm{X})$ cross sections on select targets. The reaction cross section from our calculation is represented by the black line, and our calculated $(\mathrm{n}, \mathrm{X})$ cross section by the red line. The blue line represents the $(\mathrm{n}, \mathrm{X})$ cross section from the RADCHEM database.

species, have been measured. These measured cross sections should be used in lieu of any theory calculation in UGT analysis.

\subsubsection{Destruction cross sections}

In Figure 17 we present select $(\mathrm{n}, \mathrm{X})$ cross sections, calculated via the method of section 2.7 . Additional $(\mathrm{n}, \mathrm{X})$ cross sections can be seen on the plots in Appendix D. The targets represented in Figure 17 were chosen because (a) our calculated $(\mathrm{n}, \mathrm{X})$ cross section was greater than $1 \mathrm{mb},(\mathrm{b})$ an $(\mathrm{n}, \mathrm{X})$ reaction for the target had been included in the previous detector set, and (c) with the exception of ${ }^{91} \mathrm{Mo}$, each of these targets are one of activities measured during UGT. We have included ${ }^{91}$ Mo on the basis that it is among our larger $(n, X)$ cross sections. Because we have made a special effort to include all of the most important neutron reaction channels in this study, many of our $(n, X)$ cross sections are less than $1 \mathrm{mb}$ over the entire energy range studied.
We note that three of our $(\mathrm{n}, \mathrm{X})$ cross sections are non-existent below $15 \mathrm{MeV}$ of incident energy (recall that any $(\mathrm{n}, \mathrm{X})$ cross section less that $1 \mathrm{mb}$ is set to zero to avoid "noise" due to precision issues). The exception here is ${ }^{91} \mathrm{Mo}(\mathrm{n}, \mathrm{X})$ which is still an order of magnitude smaller than the dominant reaction channel over the entire energy range. This is representative of our $(\mathrm{n}, \mathrm{X})$ cross sections. They can, in all likelihood, be left out of any network calculations, provided that all of the other important channels are included.

On the other hand, the $(\mathrm{n}, \mathrm{X})$ cross sections in the existing RADCHEM database can be quite large, in some cases comprising upwards of $20-25 \%$ of the reaction cross section or more. For the targets shown here, we specifically note that the following important ( $>10 \mathrm{mb}$ at some given energy) channels have been grouped in with the $(\mathrm{n}, \mathrm{X})$ cross section: the (n,p), (n,np), and $(\mathrm{n}, \alpha)$ reactions on ${ }^{88} \mathrm{Y}_{g}$; the $(\mathrm{n}, \gamma),(\mathrm{n}, \mathrm{p}),(\mathrm{n}, \mathrm{np})$, and $(\mathrm{n}, \alpha)$ reactions on ${ }^{92} \mathrm{Nb}_{m 1}$; the $(\mathrm{n}, \gamma),(\mathrm{n}, 2 \mathrm{n}),(\mathrm{n}, \mathrm{p})$, and $(\mathrm{n}, \alpha)$ re- 

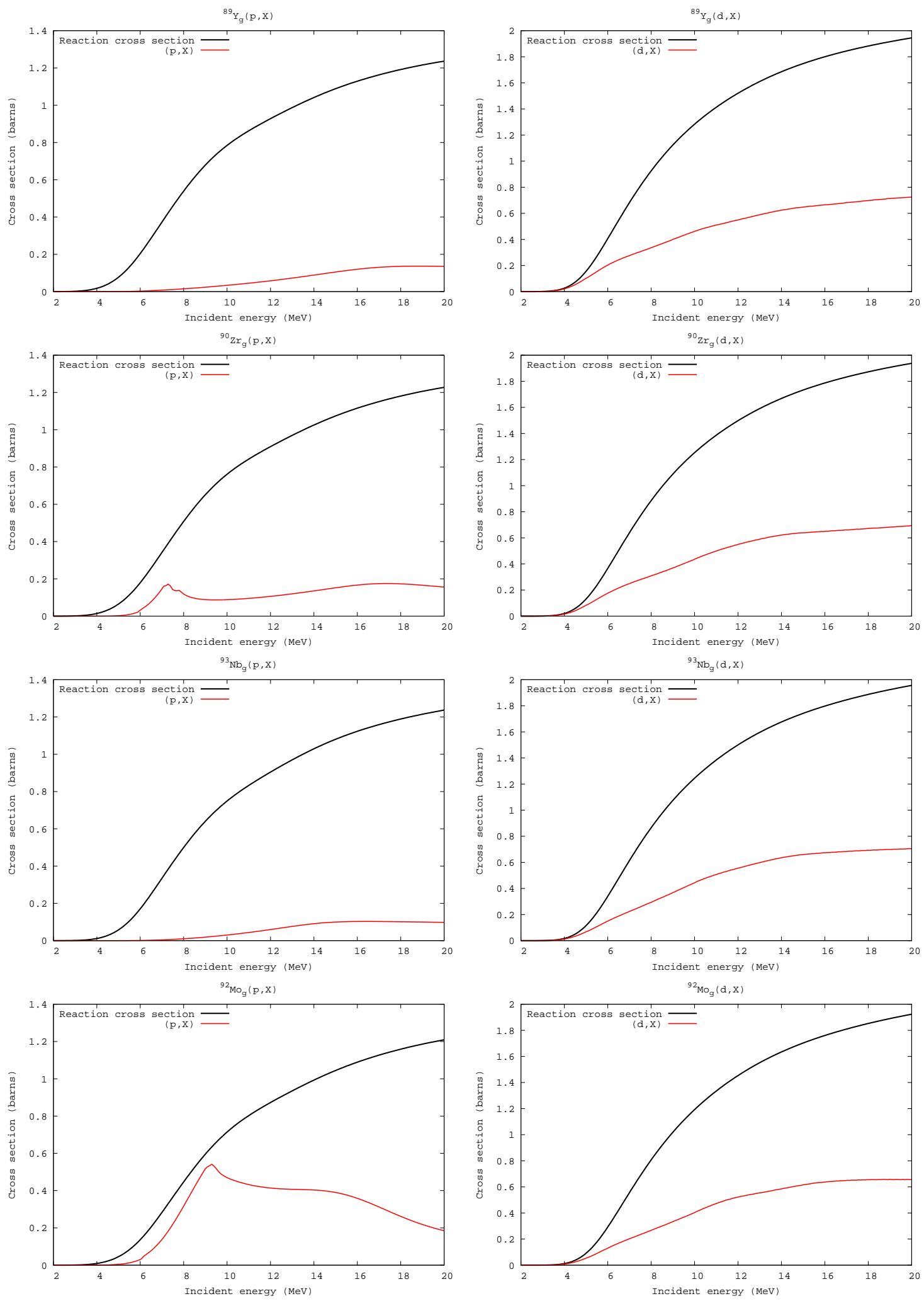

Fig. 18. - Calculated charged particle destruction cross sections on select targets. The reaction cross section from our calculation is represented by the black line, and our calculated $(\mathrm{p}, \mathrm{X})$ or $(\mathrm{d}, \mathrm{X})$ cross section by the red line. 
actions on ${ }^{93} \mathrm{Nb}_{m 1}$; the $(\mathrm{n}, \gamma),(\mathrm{n}, 2 \mathrm{n}),(\mathrm{n}, \mathrm{p})$, and $(\mathrm{n}, \mathrm{np})$ reactions on ${ }^{91} \mathrm{Mo}_{g}$. Many of these omitted channels are the dominant reaction channel over part of the energy range, as shown in Appendix D.

For incident charged particles, we have not included as many outgoing channels, and hence our charged particle destruction cross sections will be larger than those for neutrons. In Figure 18 we present our $(\mathrm{p}, \mathrm{X})$ and $(\mathrm{d}, \mathrm{X})$ cross sections, along with their respective reaction cross sections, for four of the loaded detector elements. The $(\mathrm{p}, \mathrm{X})$ reactions generally account for about $20 \%$ of the reaction cross section, except for the even- $Z$ targets at incident energies below $10 \mathrm{MeV}$, where the amount may be as large as 50-90\%. The (d,X) cross sections are fairly uniform across all targets, and account for $\sim 40 \%$ of the reaction cross section.

\subsection{Sensitivity Studies}

We now illustrate the sensitivity of our modeled results to variations in the input parameters developed in $\S 3$.

\subsubsection{Sensitivity to the Pre-Equilibrium Cross Section}

As stated in section 3.4, the simple exciton model has two free parameters which may be tuned to replicate experimental cross sections. One of these parameters affects alpha emission only and will be studied in a later section. The other parameter, $\langle F M\rangle$, scales the average effective matrix element for residual interactions. We have determined that a value of $\langle F M\rangle=250$ $\mathrm{MeV}$ optimally replicates measured cross sections in this region of interest.

The choice of $\langle F M\rangle=250 \mathrm{MeV}$ is based primarily on $(\mathrm{n}, 2 \mathrm{n})$ cross sections on various isotopes of yttrium and zirconium. Initial comparisons between measured data and cross sections modeled with varying values of $\langle F M\rangle$ indicated that this parameter should be somewhere between 200 and $300 \mathrm{MeV}$. To improve the estimate, we appealed to critical $(\mathrm{n}, 2 \mathrm{n})$ activation cross sections found in the RADCHEM library. Specifically, we attempted to match the RADCHEM ${ }^{89} \mathrm{Y}(\mathrm{n}, 2 \mathrm{n}){ }^{88} \mathrm{Y}$ and ${ }^{90} \mathrm{Zr}(\mathrm{n}, 2 \mathrm{n}){ }^{89} \mathrm{Zr}$ activation cross sections at 14.1 MeV, which are $840 \mathrm{mb}$ and $632 \mathrm{mb}$, respectively. The ${ }^{89} \mathrm{Y}(\mathrm{n}, 2 \mathrm{n})^{88} \mathrm{Y}$ was best matched with $\langle F M\rangle=300 \mathrm{MeV}$, yielding a value of $826 \mathrm{mb}$ at $14.1 \mathrm{MeV}$ (1.7\% lower than the RADCHEM value, or $-1.7 \%)$. Decreasing $\langle F M\rangle$ decreases this cross
Table 4: Sensitivity of critical $14.1 \mathrm{MeV}$ (n,2n) cross sections to the $\langle F M\rangle$ parameter. The second column shows the value of the cross section (in millibarns) in the current RADCHEM library. A "*" indicates a measured cross section. The other three columns show the cross section calculated using values of $\langle F M\rangle=200,250$, and $300 \mathrm{MeV}$.

\begin{tabular}{|c|c|c|c|c|}
\hline Reaction & Lib. & 200 & 250 & 300 \\
\hline${ }^{89} \mathrm{Y}_{g}(\mathrm{n}, 2 \mathrm{n})^{88} \mathrm{Y}_{g}$ & 561 & 541 & 571 & 596 \\
\hline${ }^{89} \mathrm{Y}_{g}(\mathrm{n}, 2 \mathrm{n}){ }^{88} \mathrm{Y}_{m 1}$ & 142 & 131 & 139 & 145 \\
\hline${ }^{89} \mathrm{Y}_{g}(\mathrm{n}, 2 \mathrm{n})^{88} \mathrm{Y}_{m 2}$ & 137 & 76 & 81 & 85 \\
\hline${ }^{89} \mathrm{Y}_{g}(\mathrm{n}, 2 \mathrm{n})^{88} \mathrm{Y}$ & $840^{*}$ & 748 & 791 & 826 \\
\hline${ }^{88} \mathrm{Y}_{g}(\mathrm{n}, 2 \mathrm{n}){ }^{87} \mathrm{Y}_{g}$ & $311^{*}$ & 246 & 258 & 267 \\
\hline${ }^{8} \mathrm{Y}_{g}(\mathrm{n}, 2 \mathrm{n})^{87} \mathrm{Y}_{m 1}$ & $801^{*}$ & 775 & 815 & 845 \\
\hline${ }^{88} \mathrm{Y}_{g}(\mathrm{n}, 2 \mathrm{n}){ }^{87} \mathrm{Y}$ & $1112^{*}$ & 1021 & 1073 & 1112 \\
\hline${ }^{87} \mathrm{Y}_{g}(\mathrm{n}, 2 \mathrm{n}){ }^{87} \mathrm{Y}_{g}$ & - & 454 & 487 & 505 \\
\hline${ }^{87} \mathrm{Y}_{g}(\mathrm{n}, 2 \mathrm{n}){ }^{87} \mathrm{Y}_{m 1}$ & - & 44 & 47 & 49 \\
\hline${ }^{87} \mathrm{Y}_{g}(\mathrm{n}, 2 \mathrm{n})^{87} \mathrm{Y}$ & 471 & 498 & 535 & 554 \\
\hline${ }^{87} \mathrm{Y}_{m 1}(\mathrm{n}, 2 \mathrm{n})^{87} \mathrm{Y}_{g}$ & - & 412 & 442 & 459 \\
\hline${ }^{87} \mathrm{Y}_{m 1}(\mathrm{n}, 2 \mathrm{n})^{87} \mathrm{Y}_{m 1}$ & - & 176 & 190 & 197 \\
\hline${ }^{87} \mathrm{Y}_{m 1}(\mathrm{n}, 2 \mathrm{n})^{87} \mathrm{Y}$ & 560 & 588 & 632 & 657 \\
\hline${ }^{90} \mathrm{Zr}_{g}(\mathrm{n}, 2 \mathrm{n})^{89} \mathrm{Zr}_{g}$ & 549 & 574 & 603 & 622 \\
\hline${ }^{90} \mathrm{Zr}_{g}(\mathrm{n}, 2 \mathrm{n})^{89} \mathrm{Zr}_{m 1}$ & 83 & 70 & 73 & 76 \\
\hline${ }^{90} \mathrm{Zr}_{g}(\mathrm{n}, 2 \mathrm{n})^{89} \mathrm{Zr}$ & $632^{*}$ & 644 & 676 & 698 \\
\hline${ }^{89} \mathrm{Zr}_{g}(\mathrm{n}, 2 \mathrm{n})^{88} \mathrm{Zr}$ & $767^{*}$ & 664 & 700 & 723 \\
\hline${ }^{89} \mathrm{Zr}_{m 1}(\mathrm{n}, 2 \mathrm{n})^{88} \mathrm{Zr}$ & 896 & 892 & 937 & 969 \\
\hline${ }^{88} \mathrm{Zr}_{g}(\mathrm{n}, 2 \mathrm{n})^{87} \mathrm{Zr}_{g}$ & - & 311 & 324 & 337 \\
\hline${ }^{8} \mathrm{Zr}_{g}(\mathrm{n}, 2 \mathrm{n})^{87} \mathrm{Zr}_{m 1}$ & - & 14 & 15 & 15 \\
\hline${ }^{88} \mathrm{Zr}_{g}(\mathrm{n}, 2 \mathrm{n})^{87} \mathrm{Zr}$ & $315^{*}$ & 325 & 339 & 352 \\
\hline${ }^{87} \mathrm{Zr}_{g}(\mathrm{n}, 2 \mathrm{n})^{86} \mathrm{Zr}$ & 695 & 282 & 293 & 306 \\
\hline${ }^{87} \mathrm{Zr}_{m 1}(\mathrm{n}, 2 \mathrm{n})^{86} \mathrm{Zr}$ & - & 426 & 443 & 462 \\
\hline
\end{tabular}

section, with $\langle F M\rangle=200 \mathrm{MeV}$ giving a $14.1 \mathrm{MeV}$ cross section of $748 \mathrm{mb}(-11.0 \%)$. The second cross section, ${ }^{90} \mathrm{Zr}(\mathrm{n}, 2 \mathrm{n})^{89} \mathrm{Zr}$, was best matched using $\langle F M\rangle=200 \mathrm{MeV}$, which gave a cross section of $644 \mathrm{mb}(+1.9 \%)$. Using $\langle F M\rangle=300 \mathrm{MeV}$ increased this cross section to $698 \mathrm{mb}(+10.4 \%)$. Rather than use different $\langle F M\rangle$ for different elements (we wish to maintain consistency in the model parameters for each reaction in this detector set), we settled on a value of $\langle F M\rangle=250$ $\mathrm{MeV}$, which resulted in $14.1 \mathrm{MeV}$ cross sections of $791 \mathrm{mb}$ for ${ }^{89} \mathrm{Y}(\mathrm{n}, 2 \mathrm{n})^{88} \mathrm{Y}(-5.8 \%)$ and $676 \mathrm{mb}$ for ${ }^{90} \mathrm{Zr}(\mathrm{n}, 2 \mathrm{n})^{89} \mathrm{Zr}(+7.0 \%)$. The cross section (in mb) obtained for other critical $(n, 2 n)$ reactions at 14.1 $\mathrm{MeV}$ in the yttrium and zirconium detector sets, along with the RADCHEM values, can be seen in Table 4. In this table, cross sections not specifying a final state are activation cross sections, and those not included in the existing RADCHEM detector sets are indicated by dashes. 

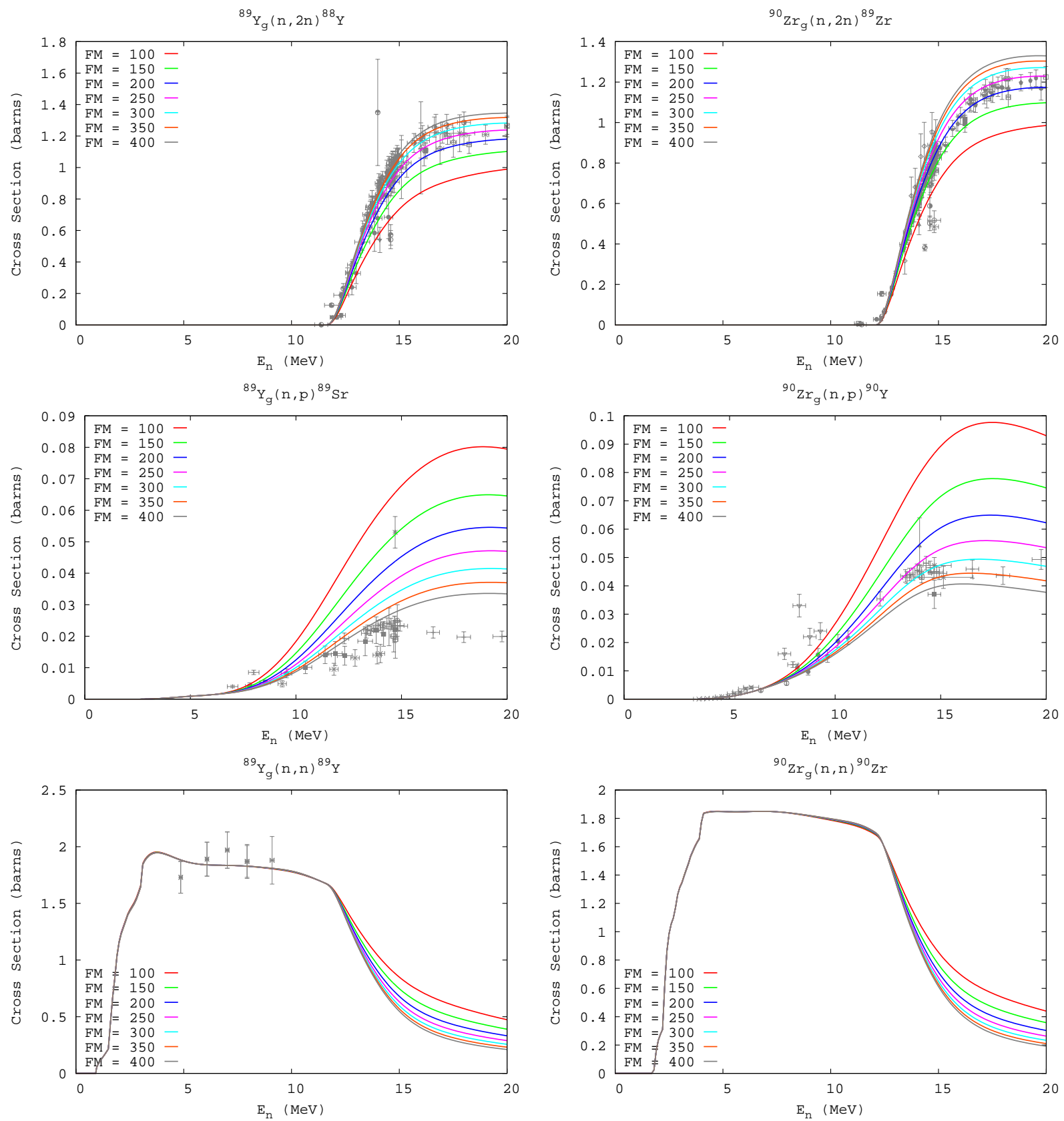

Fig. 19.- Sensitivity of select activation cross sections to the $\langle F M\rangle$ parameter. The activation cross section data (gray data points) are taken from (EXFOR 2006).

We also present in Figure 19 the sensitivity of other select neutron-induced cross sections, over the entire modeled energy range, to the $\langle F M\rangle$ parameter. As a percentage of the cross section, the sensitivity is greatest for the (n,p) reactions, followed by $(\mathrm{n}, 2 \mathrm{n})$ and $\left(\mathrm{n}, \mathrm{n}^{\prime}\right)$. However, it is worthwhile to note that the $(\mathrm{n}, \mathrm{p})$ cross section is also considerably smaller than the other two channels, so one might expect the sensitivity to be greater.

\subsubsection{Sensitivity to the Alpha Preformation Pa- rameter}

The other free parameter in the exciton model which we tune to match measured cross section data describes the tendency of nucleons to preform alpha clusters in the nucleus. This parameter, $\phi$, is expected to have a value somewhere between 0.1 to 0.8. In Figure 20 we present the sensitivity of select $(\mathrm{n}, \alpha)$ cross sections to this parame- 

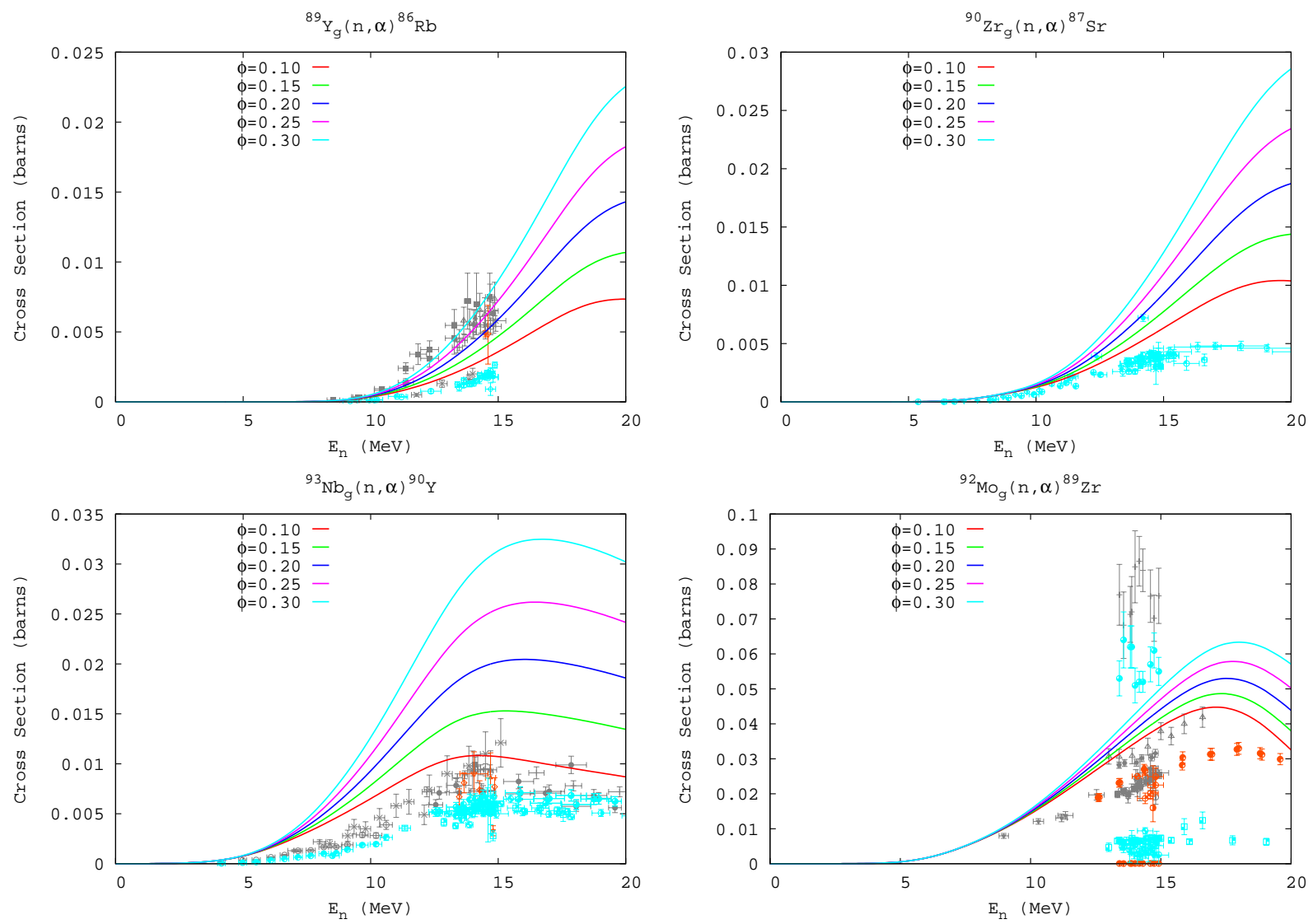

Fig. 20.- Sensitivity of select activation cross sections to the alpha preformation parameter. The cross section data (gray, orange, and cyan representing activation, ground state, and isomer data, respectively) are taken from (EXFOR 2006).

ter. All of the modeled cross sections presented in this figure are activation cross sections. The data leading to the ground state and isomers has been left on the plot, to allow a comparison with data to be made for the ${ }^{90} \mathrm{Zr}$ target. These comparisons, along with additional comparisons not shown in this report, justify a choice of $\phi=0.2$. Because the alpha channel is small compared to other exit channels, the effect on other cross sections, such as $(\mathrm{n}, 2 \mathrm{n})$ and $(\mathrm{n}, \mathrm{p})$ is negligible, and the only other calculated reaction channel that will show any sensitivity to this parameter is the $(\mathrm{n}, \mathrm{n} \alpha)$ channel.

\subsubsection{Sensitivity to the Level Density - Shell Correction Systematic}

The systematic presented in Figure 8 represents a least squares fit to shell corrections $(\delta W)$ derived from known resonance spacings, assuming a specific form for other Fermi gas level density parameters (§3.3). The error bars correspond to errors in the measured resonance spacings, obtained by de- riving shell corrections from the upper and lower limits of the measurement. The average size of these error bars is $0.60 \mathrm{MeV}$, with about half of them of them being smaller than $0.5 \mathrm{MeV}$.

In Figure 21 we investigate the effect of varying the shell corrections up or down by $1 \mathrm{MeV}$. In each case, the matching energy $E_{x}$ was refit to the spectroscopic data. The cross section obtained using our preferred shell correction is shown in red, and the cross section calculated using the $\pm 1 \mathrm{MeV}$ variations are shown in black. The activation cross section data is obtained from (EXFOR 2006).

The changes are most significant in the capture cross sections, with the variations at low incident energies being as large as $\sim 35 \%$. These changes are due primarily to the corresponding adjustments in the normalization of the photon transmission functions (see Section 3.2.3). On the other hand, the $(\mathrm{n}, 2 \mathrm{n})$ cross sections exhibit almost no sensitivity to changes in the shell correction. The $(n, p)$ cross sections also exhibit very 

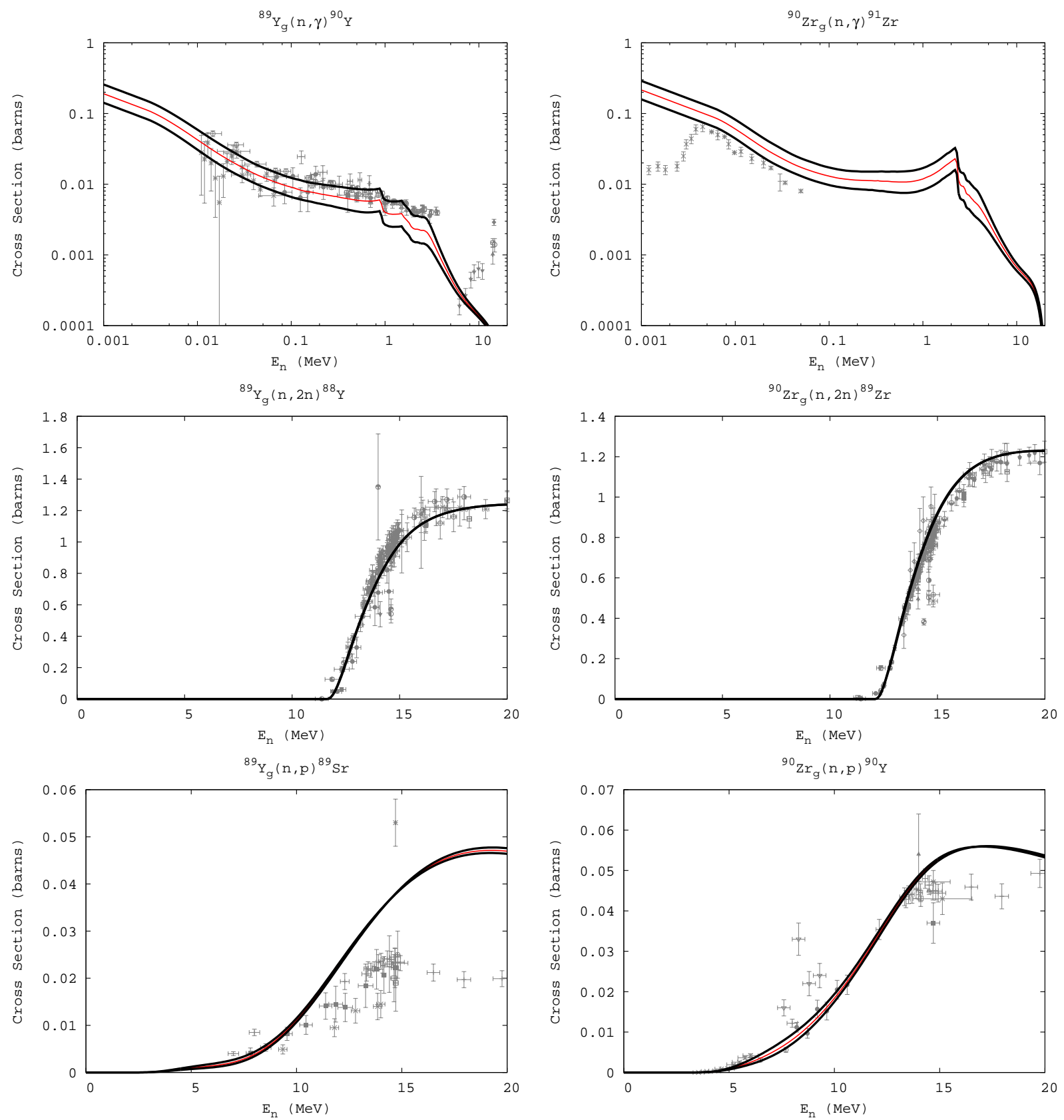

Fig. 21.- Sensitivity to variations in the level density shell correction $\delta W$.

little sensitivity. At high incident energies, the effects of these variations are expected to be minimal, since the shell correction is damped and the level density parameter approaches its asymptotic value.

We note further that all of the shell corrections (including those for the compound nucleus and each possible exit channel) have been enhanced or reduced in these calculations. The effect of varying an individual shell correction has not been inves- tigated.

\subsubsection{Sensitivity to the Normalization of the $\gamma$ - ray Transmission Coefficient}

Figure 22 shows the sensitivity of select capture cross sections to a $\pm 30 \%$ change to the value of the average s-wave photon width used to normalize the gamma-ray transmission coefficients (§3.2.3., Figure 7). The solid red line represents the cross section modeled with our adopted value for the radia- 

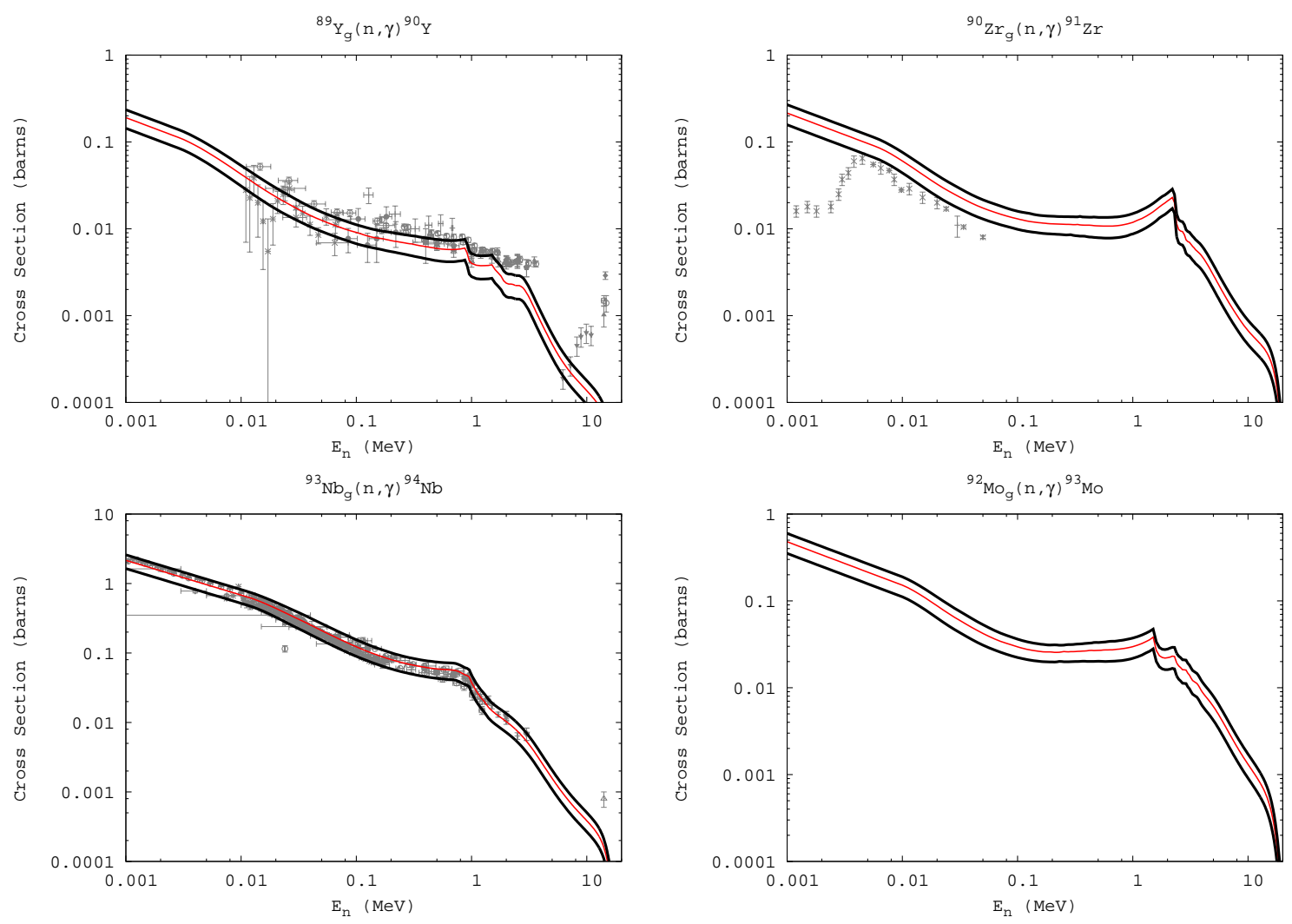

Fig. 22.- Sensitivity to a $\pm 30 \%$ adjustment of the experimental s-wave average photon width $\Gamma_{\gamma}$.

tion width, and the black lines represent cross sections modeled with a $30 \%$ variation in the adopted values. The $30 \%$ variation translates into a nearly identical change $(\sim 26 \%)$ in the cross section from $10 \mathrm{keV}$ to $1 \mathrm{MeV}$. Other neutron induced cross sections do not exhibit any sensitivity to this parameter since $\mathrm{T}_{\gamma}$ only enters into Eq. [1] in the denominator. In general, for capture reactions, the smaller of the two transmission coefficients in the $\mathrm{HF}$ numerator will be the one that determines the cross section, especially if it is much smaller. This is always the case with photon vs. particle widths. The mean error associated with the experimental values of the gamma ray strength function for isotopes with charge $34 \leq Z \leq 46$ is $21.0 \%$ with a standard deviation of $9.8 \%$. Hence, the uncertainties related to the gamma ray strength function in the capture cross sections is likely less than that shown in Figure 22.

\subsubsection{Sensitivity to the Inclusion of Width Fluc- tuation Corrections}

We adopt the Moldauer model of the width fluctuation correction (WFC) as embodied in the STAPRE code. Figure 23 shows the affect for the activation neutron capture and inelastic scattering cross sections for select targets both with (solid red line) and without (solid blue line) WFC. As expected a decrease in both channels is noticeable. For capture reactions the decrease is less than $20 \%$ below $100 \mathrm{keV}$. When the projectile energy increases, the capture cross section declines rapidly and the elastic enhancement vanishes. For the inelastic scattering, the correction is most significant between 1-4 MeV incident energy. Other channels that are open below a few $\mathrm{MeV}$ of incident energy may also exhibit sensitivity to width fluctuation corrections.

\subsection{Calculated Channels by Target}

In Appendix D we show all of the calculated neutron and charged particle induced channels leading out of the ground state for select targets of yttrium, zirconium, niobium, and molybdenum. The plots are grouped by common neutron numbers, with the neutron induced reactions on the left panes and the charged particle induced reactions on the right panes. In general, variations in a given cross section from target to target are small, although odd-even effects are apparent. 

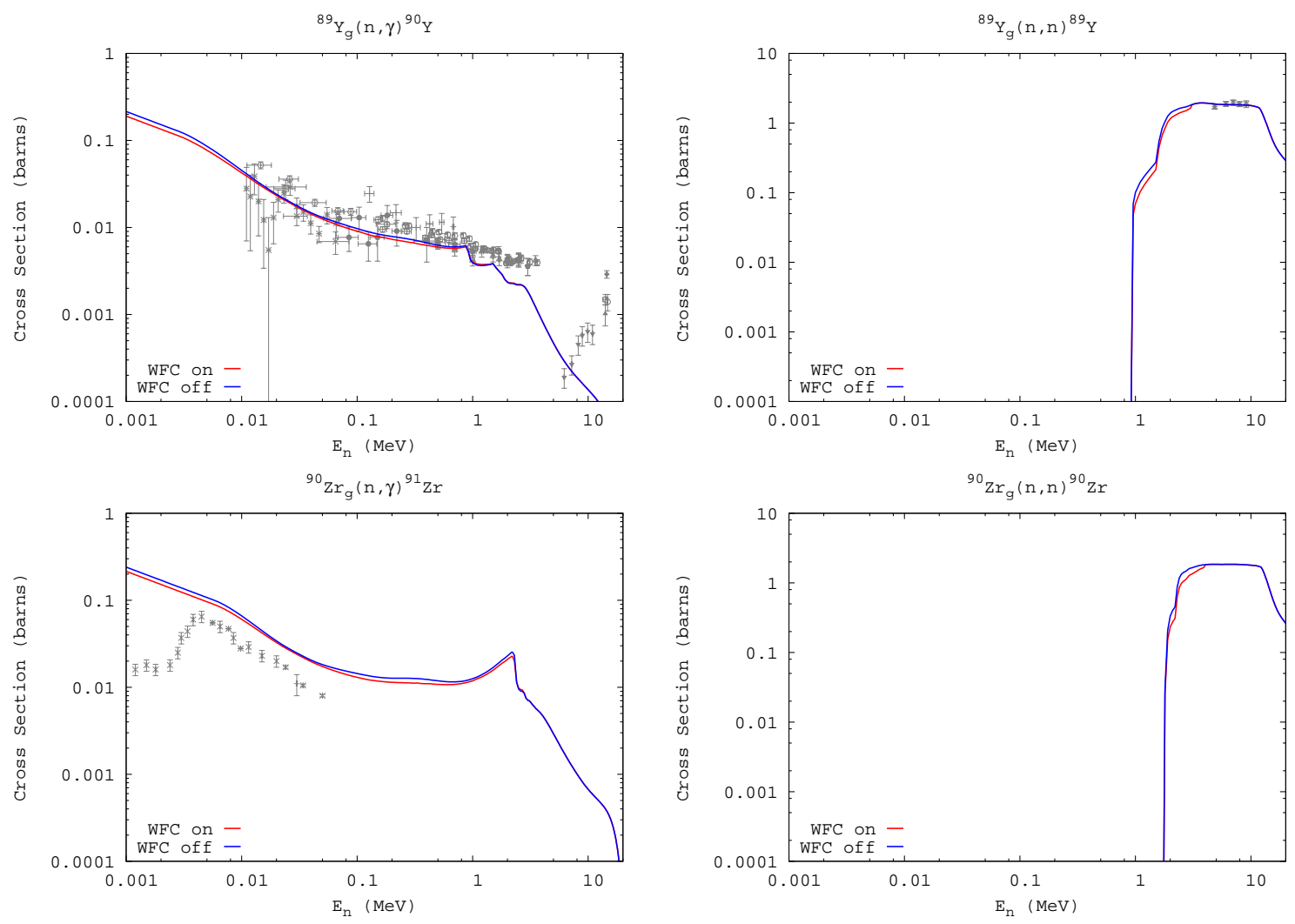

Fig. 23.- Sensitivity to inclusion or exclusion of width fluctuation corrections $W$.

These plots aid the evaluation of which reaction channels are the most important. For neutrons with low incident energies, the capture reaction is usually dominant, though the $(\mathrm{n}, \mathrm{p})$ cross section may be larger for proton rich targets. At energies around $14 \mathrm{MeV}$ the $(\mathrm{n}, 2 \mathrm{n})$ reaction is usually dominant. Occasionally (and again for proton rich species) the (n,np) reaction may also be relatively large. For the charged particle reactions, each of the calculated channels are relatively important, though which channel dominates varies with the incident energy.

Of course the particle fluences play a dominant role in determining the most important cross sections for RADCHEM. More specific details will be disclosed in a classified analysis presented in a subsequent paper.

\section{Conclusions}

We have developed new neutron and charged particle induced cross section detector sets for radiochemical diagnostics of isotopes in the region $38 \leq Z \leq 45$. The theory and implementation of the Hauser-Feshbach model were described ( $(2)$, along with the details of the local systematics used to create a set of input parameters that reflect the latest available experimental data in the local region of interest $(\S 3)$. Modeled cross sections were compared to available experimental cross sections for the loaded detector elements, as well as other stable targets in the region. Sensitivity to reasonable variations in the input models and parameters was explored (§4.2).

Overall we consider the modeling effort to be quite successful, as our calculated cross sections agree favorably with experimentally measured ones in this region of interest. In particular, we have demonstrated an ability to calculate $(\mathrm{n}, 2 \mathrm{n})$ cross sections to about $7 \%$ accuracy (Section 4.1.3), and (n, $\gamma$ ) cross sections to within roughly $30 \%$ accuracy (Sections 4.1.1 and 4.1.2). We also provide suggested normalizations to our $(\mathrm{n}, 2 \mathrm{n})$ and $(\mathrm{n}, \gamma)$ capture cross sections on stable targets to bring them into agreement (in an average sense) with the body of experimental data that exists. For charged-particle reactions we achieved an accuracy typically within $15-25 \%$.

In our attempts to model cross sections it should be kept in mind that we are consider- 
ing compound nuclear systems for which the important input parameters to our reaction model (e.g. those that affect level densities and photontransmission coefficients) are often determined by normalization to experimental data (e.g. from resonance analysis), and so one would expect comparisons to measured capture cross sections to be good. Since these compound nuclei often bracket the systems of most interest to us, namely those which account for the dominant destruction reactions, our systematics should reasonably provide for similar agreement.

This work was performed under the auspices of the U.S. Department of Energy by the University of California Lawrence Livermore National Laboratory under contract W-7405-ENG-48. 


\section{REFERENCES}

Arthur, E.D., "Calculation of neutron cross sections on isotopes of yttrium and zirconium", LA-7789-MS, LANL (1980).

Audi, G., Wapstra, A.H., and Thibault, C., "The AME2003 atomic mass evaluation (II). Tables, graphs, and references", Nuc. Phys. A 729, 337 (2003).

Avrigeanu, V., Hodgson, P.E., and Avrigeanu, M., "Global optical potentials for emitted alpha particles", Phys. Rev. C 49, 2136 (1994).

Avrigeanu, M. and Avrigeanu, V., "Recent improvements of the STAPRE-H95 preequilibrium and statistical model code", Report NP-86-1995, IPNE-Bucharest (1995). http://www.nea.fr/abs/html/iaea0971.html

Bao, Z. Y., Beer, H., Kappeler, F., Voss, F., \& Wisshak, K., "Neutron cross sections for nucleosynthesis studies", Atomic Data \& Nuclear Data Tables, 76, 70 (2000).

Bauer, R., private communication (2003).

Beer, H., Voss, F., \& Winters, R. R., "On the calculation of Maxwellian-averaged capture cross sections", ApJS, 80, 403 (1992).

Belgya, T., Bersillon, O., Copote Noy, R., Fukahori, T., Zhigang, G., Goriely, S., Herman, M., Ignatyuk, A.V., Kailas, S., Koning, A.J., Oblozinsky, P., Plujko, V, and Young, P.G., "Handbook for calculations of nuclear reaction data, RIPL-2", IAEA, Vienna (2005).

Bohr, A., \& Mottelson, B. Nuclear Structure Vol. 1, Single-Particle Motion, World Scientific, 1998

Chadwick, M., "The GNASH statistical model code", 1998. http://www.nea.fr/abs/html/psr-0125.html

Cline, C. K., "The Pauli exclusion principle in pre-equilibrium decay", Nucl. Phys. A195, 353 (1972).

Cline, C.K., and Blann, M., "The pre-equilibrium statistical model: description of the nuclear equilibration process and parameterization of the model", Nuc. Phys. A 172, 225 (1971).

Experimental Nuclear Reaction Data File, Brookhaven National Laboratory, US Dept. of Energy (2006). http://www.nndc.bnl.gov/exfor3/

Gardner, D.G., "Model calculations as one means of satisfying the neutron cross section requirements of the ctr program", Invited paper presented at the Conference on Nuclear Cross Sections and Technology, Washington, D.C., March 3-7, 1975.

Gilbert, A. and Cameron, A.G.W., "A composite nuclear-level density formula with shell corrections", Can. J. Phys., 43, 1446 (1965).

Hauser, W. and Feshbach, H., "The inelastic scattering of neutrons", Phys. Rev. 87, 366 (1952).

Hilaire, S., Lagrange, Ch., and Koning, A. J., "Comparisons between various width fluctuation correction factors for compound nucleus reactions" Ann. of Phys. 306, 209 (2003).

Hoffman, R. D., Rauscher, T., Woosley, S. E. \& Thielemann, F.-K., "The reaction rate sensitivity of nucleosynthesis in Type II supernovae", ApJ, 521, 735 (1999).

Hoffman, R.D., Dietrich, F.S., Bauer, R., Kelley, K., and Mustafa, M.G., "Neutron and charged-particle induced cross sections for radiochemistry in the region of bromine and krypton", UCRL-TR-205563, LLNL (2004a).

Hoffman, R.D., Dietrich, F.S., Bauer, R., Kelley, K., and Mustafa, M.G., "Neutron and charged-particle induced cross sections for radiochemistry in the region of iodine and xenon", UCRL-TR-206721, LLNL (2004b). 
Hoffman, R.D., Kelley, K., Dietrich, F.S., Bauer, R., and Mustafa, M.G., "Neutron and charged-particle induced cross sections for radiochemistry in the region of samarium, europium and gadolinium", UCRLTR-211558, LLNL (2004c).

Hofmann, H.M., Richert, J., Tepel, J. W., and Weidenmuller, H.A., "Direct reactions and Hauser-Feshbach theory" Ann. of Phys. 90, 403 (1975).

Iljinov, A.S., Mebel, M.V., Bianchi, N., De Sanctis, E., Guaraldo, C., Lucherini, V., Muccifora, V., Polli, E., Reolon, A.R., and Rossi, P., "Phenomenological statistical analysis of level densities, decay widths and lifetimes of excited nuclei", Nucl. Phys. A 543, 517 (1992).

Keisch, B., "Yield ratios of isomers produced by neutron activation", Phys. Rev. 129, 769 (1963).

Kelley, K., Hoffman, R.D., Dietrich, F.S., Bauer, R., and Mustafa, M.G., "'Neutron and charged-particle induced cross sections for radiochemistry for isotopes of scandium, titanium, vanadium, chromium, manganese, and iron", UCRL-TR-211668, LLNL (2005).

Kelley, K., Hoffman, R.D., Dietrich, F.S., and Mustafa, M.G., "Neutron induced cross sections for radiochemistry for isotopes of arsenic", UCRL-TR-218181, LLNL (2006).

Kelley, K., Hoffman, R.D., Dietrich, F.S., and Mustafa, M.G., "Neutron induced cross sections for radiochemistry for isotopes of nickel, copper, and zinc", UCRL-TR-221759, LLNL (2006).

Koning, A.J., \& Delaroche, J.P., "Local and global nucleon optical models from 1 keV to $200 \mathrm{MeV}$ ", Nucl. Phys. A713, 231 (2003).

Kopecky, J. and Uhl, M., "Test of gamma-ray strength functions in nuclear reaction model calculations", Phys. Rev. C 41, 1941 (1990).

Lohr, J.M. and Haeberli, W., "Elastic scattering of 9-13 MeV vector polarized deuterons", Nuc. Phys. A 232, 381 (1974).

Mahaux, C. and Weidenmüller, H.A., "Recent developments in compound-nucleus theory", Ann. Rev. Part. Nucl. Sci. 29, 1 (1979).

Milazzo-Colli, L., and Braga-Marcazzan, G.M., " $\alpha$-emission by pre-equilibrium processes in (n, $\alpha$ ) reactions", Nuc. Phys. A 210, 297 (1973).

Moldauer, P. A., "Evaluation of the fluctuation enhancement factor" Phys. Rev. C 14, 764 (1976).

Möller, P., Nix, J.R., Myers, W.D., and Swiatecki, W.J., "Nuclear ground-state masses and deformations" At. Data \& Nuc. Data Tables 59, 185 (1995).

Mughabghab, S.F., Divadeenam, M., and Holden, N.E., Neutron Cross Sections, Vols. 1 and 2, Academic Press (1981).

Mustafa, M.G., West, H.I., O'Brien H., Lanier, R.G., Benhamou, M., and Tamura, T., "Measurement and a direct-reaction plus Hauser-Feshbach analysis of ${ }^{89} \mathrm{Y}(\mathrm{p}, \mathrm{n}){ }^{89} \mathrm{Zr},{ }^{89} \mathrm{Y}(\mathrm{p}, 2 \mathrm{n}){ }^{88} \mathrm{Zr}$, and ${ }^{89} \mathrm{Y}(\mathrm{p}, \mathrm{pn})^{88} \mathrm{Y}$ reactions up to $40 \mathrm{MeV}$ ", Phys. Rev. C 38, 1624 (1998).

Nethaway, D.R., "The cross-section sets used with the Watusi program", A-Division memo, LLNL (5 Nov. 1998).

Prestwood, R.J., Thomas, K.W., Nethaway, D.R., and Smith, N.L., "Measurement of 14-MeV neutron cross sections for ${ }^{88} \mathrm{Zr}$ and ${ }^{88}$ Y", Phys. Rev. C 29, 805 (1984).

Rauscher, T., Thielemann, F.-K., and Kratz, K.-L., "Nuclear level density and the determination of thermonuclear rates for astrophysics", Phys. Rev. C 56, 1613 (1997). 
Raynal, J. "ECIS96", Proceedings of the Specialists' Meeting on the Nucleon Nucleus Optical Model up to $200 \mathrm{MeV}, 13-15$ November 1996, Bruyeres-le-Chatel, France. http://www.nea.fr/html/science/om200/raynal.pdf

Reffo, G., ICTP Lecture Series, 17 Jan. - 10 Mar., 1978, Trieste.

Tuli, J.K., "Nuclear wallet cards", 6th Ed., Brookhaven National Laboratory, U.S. Dept. of Energy (2000).

Udagawa, T., \& Tamura, T., "Derivation of breakup-fusion cross sections from the optical theorem", Phys. Rev. C 24, 1348 (1981).

Uhl, M., and Strohmaier, B., "STAPRE: A computer code for particle induced activation cross sections and related quantities", IRK-Vienna Report IRK-76/01 (1976, Upd. 1978).

Vonach, H., "User's manual for the code STAPRE as implemented at Lawrence Livermore National Laboratory", UCID-19549, LLNL (1982).

Wapstra, A.H., Audi, G., and Thibault, C., "The AME2003 atomic mass evaluation (I). Evaluation of input data, adjustment procedures", Nuc. Phys. A 729, 129 (2003).

West, H.I., Lanier, R.G., Mustafa, M.G., Nuckolls, R.M., Nagle, R.J., O’Brien, H., Frehaut, J., Adam, A., and Philis, C., "Some light-ion excitation-function measurements on titanium, yttrium, and europium, and associated results", UCRL-ID-115738, LLNL (1993).

Williams, F.C. Jr., "Intermediate state transition rates in the Griffin model", Phys. Lett. 31B, 184 (1970).

Woosley, S.E., Fowler, W.A., Holmes, J.A., and Zimmerman, B.A., "Tables of thermonuclear reaction rate data for intermediate mass nuclei", Orange aid preprint series in nuclear, atomic and relativistic astrophysics (1975).

This 2-column preprint was prepared with the AAS LATEX macros v5.0. 


\section{A. Cross Sections Included in the Detector Sets}

\section{A.1. Cross Sections in the Existing RADCHEM Detector Sets}

Cross sections for the following reactions are provided in the existing RADCHEM data sets YT0585, YT0488, Zr0982, Nb0179, and Mo1278. The majority of these reactions were calculated between 1972 and 1988. A few have been scaled to match measured cross sections at or around $14.1 \mathrm{MeV}$ of incident energy. Seven of the charged-particle reaction in set YT0488 are measured. See (Nethaway 1998) for more details.

Table 5: Cross sections available in existing RADCHEM detector sets

\begin{tabular}{|c|c|c|c|c|}
\hline${ }^{86} \mathrm{Y}:$ & ${ }^{86} \mathrm{Y}(\mathrm{n}, \gamma)^{87 g} \mathrm{Y}^{\mathrm{a}}$ & ${ }^{86} \mathrm{Y}(\mathrm{n}, \gamma)^{87 m} \mathrm{Y}^{\mathrm{a}}$ & ${ }^{86} \mathrm{Y}(\mathrm{n}, 2 \mathrm{n})^{85} \mathrm{Y}^{\mathrm{a}}$ & ${ }^{86} \mathrm{Y}(\mathrm{n}, \mathrm{X})^{\mathrm{a}}$ \\
\hline${ }^{87 g} \mathrm{Y}:$ & $\begin{array}{l}{ }^{87 g} \mathrm{Y}(\mathrm{n}, \gamma)^{88 g} \mathrm{Y}^{\mathrm{b}} \\
{ }^{87 g} \mathrm{Y}(\mathrm{n}, \mathrm{X}){ }^{\mathrm{b}}\end{array}$ & ${ }^{87 g} \mathrm{Y}(\mathrm{n}, \gamma)^{88 m} \mathrm{Y}^{\mathrm{b}}$ & ${ }^{87 g} \mathrm{Y}\left(\mathrm{n}, \mathrm{n}^{\prime}\right)^{87 m} \mathrm{Y}^{\mathrm{b}}$ & ${ }^{87 g} \mathrm{Y}(\mathrm{n}, 2 \mathrm{n})^{86} \mathrm{Y}^{\mathrm{b}}$ \\
\hline${ }^{87 m} \mathrm{Y}:$ & $\begin{array}{l}{ }^{87 m} \mathrm{Y}(\mathrm{n}, \gamma)^{88 g} \mathrm{Y}^{\mathrm{b}} \\
{ }^{87 m} \mathrm{Y}(\mathrm{n}, 2 \mathrm{n})^{86} \mathrm{Y} \text { b }\end{array}$ & $\begin{array}{l}{ }^{87 m} \mathrm{Y}(\mathrm{n}, \gamma)^{88 m 1} \mathrm{Y}^{\mathrm{b}} \\
{ }^{87 m} \mathrm{Y}(\mathrm{n}, \mathrm{X}){ }^{\mathrm{b}}\end{array}$ & ${ }^{87 m} \mathrm{Y}(\mathrm{n}, \gamma)^{88 m 2} \mathrm{Y}^{\mathrm{b}}$ & ${ }^{87 m} \mathrm{Y}\left(\mathrm{n}, \mathrm{n}^{\prime}\right)^{87 g} \mathrm{Y}^{\mathrm{b}}$ \\
\hline${ }^{88 g} \mathrm{Y}:$ & $\begin{array}{l}{ }^{88 g} \mathrm{Y}(\mathrm{n}, \gamma)^{89 g} \mathrm{Y}^{\mathrm{b}} \\
{ }^{88 g} \mathrm{Y}(\mathrm{n}, 2 \mathrm{n})^{87 g} \mathrm{Y}^{\mathrm{b}, \mathrm{g}} \\
{ }^{88 g} \mathrm{Y}(\mathrm{p}, 2 \mathrm{n})^{87} \mathrm{Zr}^{\mathrm{c}}\end{array}$ & $\begin{array}{l}{ }^{88}{ }^{8} \mathrm{Y}(\mathrm{n}, \gamma)^{89 m} \mathrm{Y}^{\mathrm{b}} \\
{ }^{88} \mathrm{Y}(\mathrm{n}, 2 \mathrm{n})^{87 m} \mathrm{Y}^{\mathrm{b}, \mathrm{g}} \\
{ }^{88} \mathrm{Y}(\mathrm{d}, 2 \mathrm{n})^{88} \mathrm{Zr}^{\mathrm{c}}\end{array}$ & $\begin{array}{l}{ }^{88 g} \mathrm{Y}\left(\mathrm{n}, \mathrm{n}^{\prime}\right)^{88 m 1} \mathrm{Y}^{\mathrm{b}} \\
{ }^{88 g} \mathrm{Y}(\mathrm{n}, \mathrm{X}) \mathrm{b}^{8} \\
{ }^{88 g} \mathrm{Y}(\mathrm{d}, 3 \mathrm{n})^{87} \mathrm{Zr}^{\mathrm{c}}\end{array}$ & $\begin{array}{l}{ }^{88 g} \mathrm{Y}\left(\mathrm{n}, \mathrm{n}^{\prime}\right)^{88 m 2} \mathrm{Y}^{\mathrm{b}} \\
{ }^{88 g} \mathrm{Y}(\mathrm{p}, \mathrm{n})^{88} \mathrm{Zr}^{\mathrm{c}}\end{array}$ \\
\hline${ }^{88 m 1} \mathrm{Y}:$ & $\begin{array}{l}{ }^{88 m 1} \mathrm{Y}(\mathrm{n}, \gamma)^{89 g} \mathrm{Y}^{\mathrm{b}} \\
{ }^{88 m 1} \mathrm{Y}(\mathrm{n}, 2 \mathrm{n})^{87 g} \mathrm{Y}^{\mathrm{b}, \mathrm{g}} \\
{ }^{88 m 1} \mathrm{Y}(\mathrm{p}, 2 \mathrm{n})^{87} \mathrm{Zr}^{\mathrm{c}}\end{array}$ & $\begin{array}{l}{ }^{88 m 1} \mathrm{Y}(\mathrm{n}, \gamma)^{89 m} \mathrm{Y}^{\mathrm{b}} \\
{ }^{88 m 1} \mathrm{Y}(\mathrm{n}, 2 \mathrm{n})^{87 m} \mathrm{Y}^{\mathrm{b}, \mathrm{g}} \\
{ }^{88 m 1} \mathrm{Y}(\mathrm{d}, 2 \mathrm{n})^{88} \mathrm{Zr}^{\mathrm{c}}\end{array}$ & $\begin{array}{l}{ }^{88 m^{1}} \mathrm{Y}\left(\mathrm{n}, \mathrm{n}^{\prime}\right)^{88 g} \mathrm{Y}^{\mathrm{b}} \\
{ }^{88 m^{1}} \mathrm{Y}(\mathrm{n}, \mathrm{X}) \mathrm{b}^{87} \mathrm{zr}^{\mathrm{c}}\end{array}$ & $\begin{array}{l}{ }^{88 m 1} \mathrm{Y}\left(\mathrm{n}, \mathrm{n}^{\prime}\right)^{88 m 2} \mathrm{Y}^{\mathrm{b}} \\
{ }^{88 m 1} \mathrm{Y}(\mathrm{p}, \mathrm{n})^{88} \mathrm{Zr}^{\mathrm{c}}\end{array}$ \\
\hline${ }^{88 m 2} \mathrm{Y}:$ & $\begin{array}{l}{ }^{88 m 2} \mathrm{Y}(\mathrm{n}, \gamma)^{89 g} \mathrm{Y}^{\mathrm{b}} \\
{ }^{88 m 2} \mathrm{Y}(\mathrm{n}, 2 \mathrm{n})^{87 g} \mathrm{Y}^{\mathrm{b}, \mathrm{g}} \\
{ }^{88 m 2} \mathrm{Y}(\mathrm{p}, 2 \mathrm{n})^{87} \mathrm{Zr}^{\mathrm{c}}\end{array}$ & $\begin{array}{l}{ }^{88 m 2} \mathrm{Y}(\mathrm{n}, \gamma)^{89 m} \mathrm{Y}^{\mathrm{b}} \\
{ }^{88 m 2} \mathrm{Y}(\mathrm{n}, 2 \mathrm{n})^{87 m} \mathrm{Y}^{\mathrm{b}, \mathrm{g}} \\
{ }^{88 m 2} \mathrm{Y}(\mathrm{d}, 2 \mathrm{n})^{88} \mathrm{Zr}^{\mathrm{c}}\end{array}$ & $\begin{array}{l}{ }^{88 m 2} \mathrm{Y}\left(\mathrm{n}, \mathrm{n}^{\prime}\right)^{88 g} \mathrm{Y}^{\mathrm{b}} \\
{ }^{88 m 2} \mathrm{Y}(\mathrm{n}, \mathrm{X}) \mathrm{b}^{8} \mathrm{zr}^{\mathrm{c}}\end{array}$ & $\begin{array}{l}{ }^{88 m 2} \mathrm{Y}\left(\mathrm{n}, \mathrm{n}^{\prime}\right)^{88 m 1} \mathrm{Y}^{\mathrm{b}} \\
{ }^{88 m 2} \mathrm{Y}(\mathrm{p}, \mathrm{n})^{88} \mathrm{Zr}^{\mathrm{c}}\end{array}$ \\
\hline${ }^{89} g \mathrm{Y}:$ & $\begin{array}{l}{ }^{89}{ }^{g} \mathrm{Y}(\mathrm{n}, \gamma)^{90} \mathrm{Y}^{\mathrm{a}} \\
{ }^{89}{ }^{g} \mathrm{Y}(\mathrm{n}, 2 \mathrm{n})^{88 m} \mathrm{Y}^{\mathrm{a}, \mathrm{g}} \\
{ }^{89} \mathrm{Y}(\mathrm{p}, 2 \mathrm{n})^{88} \mathrm{Zr} \text { e } \\
{ }^{89} \mathrm{Y}(\mathrm{d}, 3 \mathrm{n})^{88} \mathrm{Zr}^{\mathrm{f}}\end{array}$ & $\begin{array}{l}{ }^{89}{ }^{g} \mathrm{Y}\left(\mathrm{n}, \mathrm{n}^{\prime}\right){ }^{89 m} \mathrm{Y}^{\mathrm{a}} \\
{ }^{89} \mathrm{Y}(\mathrm{n}, \mathrm{X}){ }^{\mathrm{a}} \\
{ }^{89} \mathrm{Y}(\mathrm{p}, \mathrm{np})^{88 g} \mathrm{Y}^{\mathrm{e}}\end{array}$ & $\begin{array}{l}{ }^{89}{ }^{g} \mathrm{Y}(\mathrm{n}, 2 \mathrm{n})^{88}{ }^{8} \mathrm{Y}^{\mathrm{a}, \mathrm{g}} \\
{ }^{89} \mathrm{Y}(\mathrm{p}, \mathrm{n})^{89} \mathrm{Zr}^{\text {e }} \\
{ }^{89} \mathrm{Y}(\mathrm{d}, 2 \mathrm{n})^{89} \mathrm{Zr}^{\mathrm{f}}\end{array}$ & $\begin{array}{l}{ }^{89}{ }^{g} \mathrm{Y}(\mathrm{n}, 2 \mathrm{n})^{88 m 1} \mathrm{Y}^{\mathrm{a}, \mathrm{g}} \\
{ }^{89} \mathrm{Y}(\mathrm{p}, \mathrm{n})^{89 m} \mathrm{Zr}^{\mathrm{e}} \\
{ }^{89} \mathrm{Y}(\mathrm{d}, 2 \mathrm{n})^{89 m} \mathrm{Zr}^{\mathrm{f}}\end{array}$ \\
\hline${ }^{89 m} \mathrm{Y}:$ & $\begin{array}{l}{ }^{89 m} \mathrm{Y}(\mathrm{n}, \gamma)^{90} \mathrm{Y}^{\mathrm{a}} \\
{ }^{89 m} \mathrm{Y}(\mathrm{n}, 2 \mathrm{n})^{88 m 2} \mathrm{Y}^{\mathrm{a}} \\
{ }^{89 m} \mathrm{Y}(\mathrm{p}, 2 \mathrm{n})^{88} \mathrm{Zr}^{\mathrm{c}}\end{array}$ & $\begin{array}{l}{ }^{89 m} \mathrm{Y}\left(\mathrm{n}, \mathrm{n}^{\prime}\right)^{89 g} \mathrm{Y}^{\mathrm{a}} \\
{ }^{89 m} \mathrm{Y}(\mathrm{n}, \mathrm{X}){ }^{\mathrm{a}} \\
{ }^{89 m} \mathrm{Y}(\mathrm{d}, 2 \mathrm{n})^{89} \mathrm{Zr}^{\mathrm{c}}\end{array}$ & $\begin{array}{l}{ }^{89 m} \mathrm{Y}(\mathrm{n}, 2 \mathrm{n})^{88 g} \mathrm{Y}^{\mathrm{a}} \\
{ }^{89 m} \mathrm{Y}(\mathrm{p}, \mathrm{n})^{89 g} \mathrm{Zr}^{\mathrm{c}} \\
{ }^{89 m} \mathrm{Y}(\mathrm{d}, 2 \mathrm{n})^{89 m} \mathrm{Zr}^{\mathrm{c}}\end{array}$ & $\begin{array}{l}{ }^{89 m} \mathrm{Y}(\mathrm{n}, 2 \mathrm{n})^{88 m 1} \mathrm{Y}^{\mathrm{a}} \\
{ }^{89 m} \mathrm{Y}(\mathrm{p}, \mathrm{n})^{89 m} \mathrm{Zr}^{\mathrm{c}}\end{array}$ \\
\hline${ }^{90} \mathrm{Y}:$ & ${ }^{90} \mathrm{Y}(\mathrm{n}, \gamma){ }^{91} \mathrm{Y}^{\mathrm{a}}$ & ${ }^{90} \mathrm{Y}(\mathrm{n}, 2 \mathrm{n}){ }^{89 g} \mathrm{Y}^{\mathrm{a}}$ & ${ }^{90} \mathrm{Y}(\mathrm{n}, 2 \mathrm{n})^{89 m} \mathrm{Y}^{\mathrm{a}}$ & ${ }^{90} \mathrm{Y}(\mathrm{n}, \mathrm{X})^{\mathrm{a}}$ \\
\hline${ }^{91} \mathrm{Y}:$ & ${ }^{91} \mathrm{Y}(\mathrm{n}, \gamma){ }^{92} \mathrm{Y}^{\mathrm{a}}$ & ${ }^{91} \mathrm{Y}(\mathrm{n}, 2 \mathrm{n}){ }^{90} \mathrm{Y}^{\mathrm{a}}$ & ${ }^{91} \mathrm{Y}(\mathrm{n}, 3 \mathrm{n})^{89 g} \mathrm{Y}^{\mathrm{a}}$ & ${ }^{91} \mathrm{Y}(\mathrm{n}, \mathrm{X})^{\mathrm{a}}$ \\
\hline${ }^{92} \mathrm{Y}:$ & ${ }^{92} \mathrm{Y}(\mathrm{n}, \gamma){ }^{93} \mathrm{Y}^{\mathrm{a}}$ & ${ }^{92} \mathrm{Y}(\mathrm{n}, 2 \mathrm{n})^{91} \mathrm{Y}^{\mathrm{a}}$ & ${ }^{92} \mathrm{Y}(\mathrm{n}, 3 \mathrm{n}){ }^{90} \mathrm{Y}^{\mathrm{a}}$ & ${ }^{92} \mathrm{Y}(\mathrm{n}, \mathrm{X}){ }^{\mathrm{a}}$ \\
\hline${ }^{87} \mathrm{Zr}:$ & ${ }^{87} \operatorname{Zr}(\mathrm{n}, \gamma)^{88} \mathrm{Zr} \mathrm{b}$ & ${ }^{87} \mathrm{Zr}(\mathrm{n}, 2 \mathrm{n})^{86} \mathrm{Zr} \mathrm{b}$ & ${ }^{87} \mathrm{Zr}(\mathrm{n}, \mathrm{p})^{87 g} \mathrm{Y}^{\mathrm{b}}$ & \\
\hline${ }^{88} \mathrm{Zr}:$ & $\begin{array}{l}{ }^{88} \mathrm{Zr}(\mathrm{n}, \gamma)^{89 g} \mathrm{Zr} \mathrm{b} \\
{ }^{88} \mathrm{Zr}(\mathrm{n}, \mathrm{np})^{87 g} \mathrm{Y} \text { b,g }\end{array}$ & ${ }^{8} \mathrm{Zr}(\mathrm{n}, \gamma)^{89 m} \mathrm{Zr}{ }^{\mathrm{b}}$ & ${ }^{88} \mathrm{Zr}(\mathrm{n}, 2 \mathrm{n})^{87} \mathrm{Zr}$ b,g & ${ }^{88} \mathrm{Zr}(\mathrm{n}, \mathrm{p})^{88 g} \mathrm{Y}^{\mathrm{b}}$ \\
\hline${ }^{89} \mathrm{Zr}:$ & $\begin{array}{l}{ }^{89} \mathrm{Zr}(\mathrm{n}, \gamma)^{90 g} \mathrm{Zr} \mathrm{b} \\
{ }^{89} \mathrm{Zr}(\mathrm{n}, 2 \mathrm{n})^{88} \mathrm{Zr} \text { b }\end{array}$ & $\begin{array}{l}{ }^{89}{ }^{2} \mathrm{Zr}(\mathrm{n}, \gamma)^{90 m 2} \mathrm{Zr} \mathrm{b} \\
{ }^{89} \operatorname{Zr}(\mathrm{n}, \mathrm{p})^{89 g} \mathrm{Y}^{\mathrm{b}}\end{array}$ & $\begin{array}{l}{ }^{89}{ }^{2} \mathrm{Zr}(\mathrm{n}, \gamma)^{90 m 3} \mathrm{Zr} \text { b } \\
{ }^{89} \mathrm{Zr}(\mathrm{n}, \mathrm{np})^{88 g} \mathrm{Y}^{\mathrm{b}}\end{array}$ & ${ }^{89} \mathrm{Zr}\left(\mathrm{n}, \mathrm{n}^{\prime}\right)^{89 m} \mathrm{Zr}$ b \\
\hline${ }^{89 m} \mathrm{Zr}:$ & ${ }^{89 m} \operatorname{Zr}(\mathrm{n}, \gamma)^{90 g} \mathrm{Zr}$ & ${ }^{89 m} \operatorname{Zr}(\mathrm{n}, \gamma)^{90 m 1} \mathrm{Zr}{ }^{\mathrm{b}}$ & ${ }^{89 m} \operatorname{Zr}(\mathrm{n}, \gamma)^{90 m 2} \mathrm{Zr}$ & ${ }^{89 m} \mathrm{Zr}\left(\mathrm{n}, \mathrm{n}^{\prime}\right)^{89 g} \mathrm{Zr}$ b \\
\hline
\end{tabular}

Continued on next page...

${ }^{\text {a }}$ Calculation from (Arthur 1980).

b Calculated by M. Gardner and D. Gardner between 1972 and 1985 at LLNL.

${ }^{c}$ Calculated by M. Mustafa in 1988 at LLNL.

${ }^{d}$ Calculated by M. Gardner in 1978 at LLNL.

e Measurement from (Mustafa et al. 1988).

${ }^{\mathrm{f}}$ Measurement from (West et al. 1993).

g Scaled to match measured cross section data near $14 \mathrm{MeV}$ incident energy. 
Table 5: (continued)

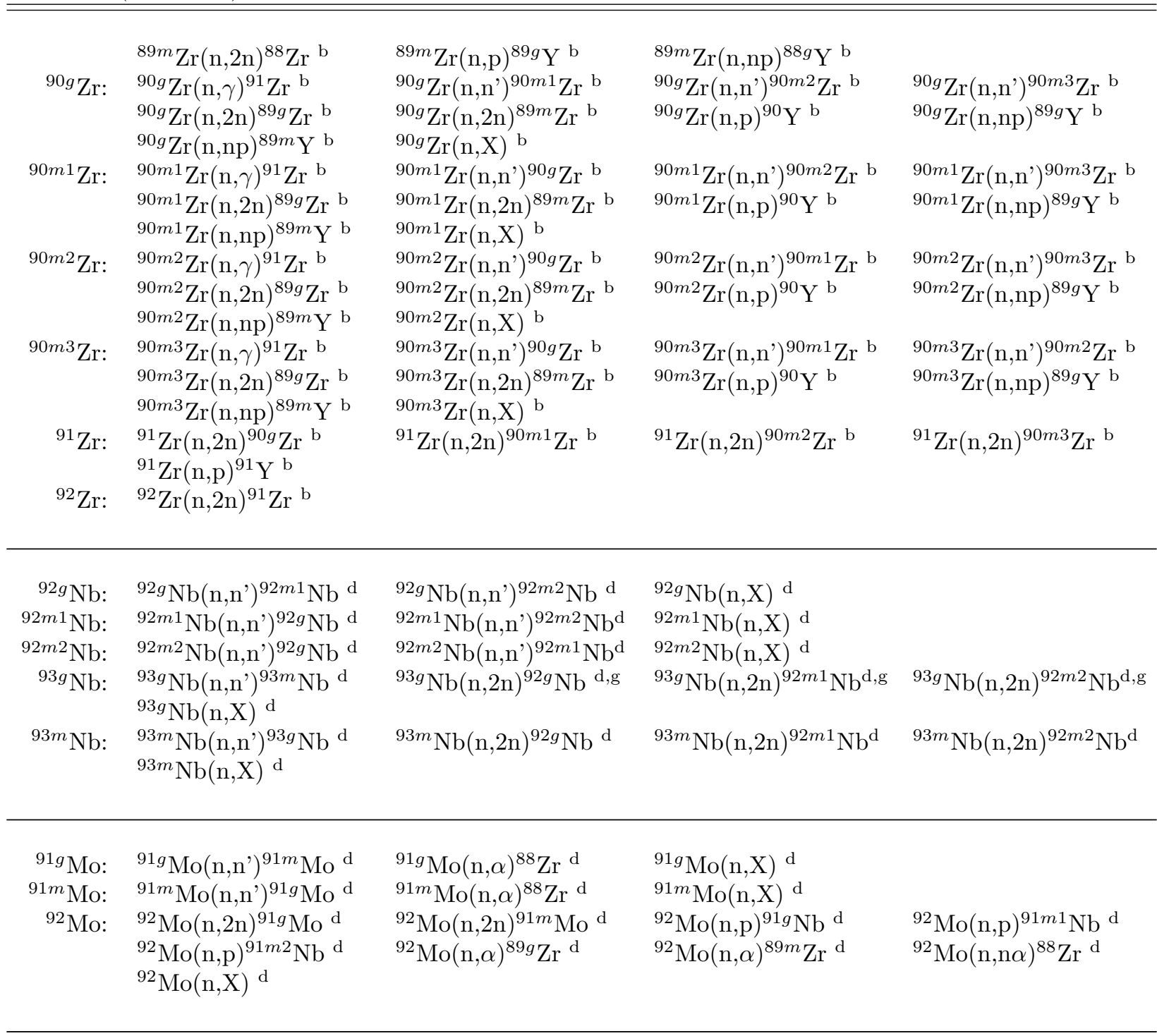

a Calculation from (Arthur 1980).

b Calculated by M. Gardner and D. Gardner between 1972 and 1985 at LLNL.

c Calculated by M. Mustafa in 1988 at LLNL.

d Calculated by M. Gardner in 1978 at LLNL.

e Measurement from (Mustafa et al. 1988).

${ }^{\mathrm{f}}$ Measurement from (West et al. 1993).

g Scaled to match measured cross section data near $14 \mathrm{MeV}$ incident energy. 


\section{A.2. Proposed New Detector Set}

Table 6 (beginning on the next page) lists the reaction channels considered in the present work.

The first column indicates the target nucleus, in its ground or isomeric state (if the state is not explicitly listed, then only a ground state exists for the target). Column two indicates the spin and parity of the target. Column four lists the half-life for radioactive targets and the natural abundance (in bold face) for stable targets. Column four lists the excitation energy for isomer targets in $\mathrm{keV}$.

For each of the targets listed, we model the reaction channels indicated in the remaining columns. Closed dots indicate that the reaction channel was also included in the existing RADCHEM data sets. Open dots indicate that the reaction channel is included only in the new set. Reaction channels have only been excluded in the event that the reaction threshold was greater than $20 \mathrm{MeV}$. The notable exception is ${ }^{100 m} \mathrm{Rh}$, for which the discrete level scheme obtained from (Belgya et al. 2005) is not complete up to the isomer energy. In cases where the residual nucleus has a long-lived isomer $\left(t_{1 / 2}>1 \mu \mathrm{s}\right)$, we model not only the activation cross section but also the cross sections leading to the ground and isomeric states. To be consistent with the RADCHEM set zr0982, we treat the first two excited states of ${ }^{90} \mathrm{Zr}$ as isomers. All reaction channels with more than one possible ordering of the outgoing particles include the sum of the cross section for all orderings, i.e. $(\mathrm{n}, \mathrm{np})$ represents $(\mathrm{n}, \mathrm{np})+(\mathrm{n}, \mathrm{pn})$.

The destruction cross sections $(\mathrm{n}, \mathrm{X}),(\mathrm{p}, \mathrm{X})$, and $(\mathrm{d}, \mathrm{X})$ have been determined by summing the other included reaction channels and subtracting the total from the reaction cross section, as described in Section 2.7 . 
Table 6: Proposed New Detector Set

\begin{tabular}{|c|c|c|c|c|c|c|c|c|c|c|c|c|c|c|c|c|c|c|c|c|c|c|c|c|c|}
\hline Target & $J^{\pi}$ & $t_{1 / 2}$ & Energy & $\stackrel{2}{\approx}$ & 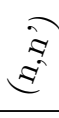 & $\underset{\approx}{\approx}$ & શ્శ & $\stackrel{2}{\Omega}$ & 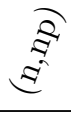 & $\underset{\Xi}{\partial}$ & 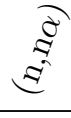 & $\underset{\Xi}{\approx}$ & $\underset{\Xi}{E}$ & $\stackrel{8}{8}$ & $\stackrel{\vartheta}{\stackrel{\vartheta}{8}}$ & $\stackrel{2}{2}$ & $\stackrel{\Xi}{\Omega}$ & $\frac{\pi}{2}$ & $\vec{Q}$ & $\underset{7}{\overparen{7}}$ & $\underset{\nabla}{\overparen{\nabla}}$ & ఫ্ণ & 尺্ & $\underset{i}{i}$ & $\underset{\mathbb{E}}{\mathbb{E}}$ \\
\hline${ }^{83} g \mathrm{Sr}$ & $7 / 2+$ & $32.41 \mathrm{~h}$ & & $\circ$ & o & ○ & & ० & ○ & ○ & ○ & ○ & ○ & ○ & ○ & ० & ० & ० & ○ & ○ & ○ & ○ & ○ & o & $\circ$ \\
\hline${ }^{83 m} \mathrm{Sr}$ & $1 / 2$ & $4.95 \mathrm{~s}$ & 259.15 & o & ० & o & & ० & ० & ० & ○ & ० & ○ & ○ & ○ & ० & ० & ० & ० & ○ & ○ & ○ & ○ & ० & ○ \\
\hline${ }^{84} \mathrm{Sr}$ & $0+$ & $0.56 \%$ & & ० & $\circ$ & ○ & & ○ & ○ & ० & $\circ$ & ○ & $\circ$ & $\circ$ & $\circ$ & $\circ$ & ○ & ○ & ○ & $\circ$ & $\circ$ & ○ & $\circ$ & ० & $\circ$ \\
\hline${ }^{85} g \mathrm{Sr}$ & $9 / 2+$ & $64.84 \mathrm{~d}$ & & ० & o & o & & o & o & o & o & o & o & o & o & o & o & o & o & o & o & ○ & o & o & ○ \\
\hline${ }^{85 m} \mathrm{Sr}$ & $1 / 2$ & $67.63 \mathrm{~m}$ & 238.66 & o & o & o & & $\circ$ & $\circ$ & $\circ$ & $\circ$ & $\circ$ & $\circ$ & ○ & o & $\circ$ & ○ & ० & $\circ$ & ○ & $\circ$ & o & $\circ$ & ० & ○ \\
\hline${ }^{86} \mathrm{Sr}$ & $0+$ & $9.86 \%$ & & $\circ$ & $\circ$ & o & & $\circ$ & $\circ$ & $\circ$ & ○ & $\circ$ & ○ & ○ & $\circ$ & $\circ$ & ० & ○ & $\circ$ & ० & ○ & $\circ$ & ○ & ० & ○ \\
\hline${ }^{87} g \mathrm{Sr}$ & $9 / 2+$ & $7.00 \%$ & & o & ० & ० & 0 & ० & ० & ० & ○ & ० & ○ & ○ & ○ & ○ & ० & ० & ० & ○ & ○ & ○ & ○ & ० & ○ \\
\hline${ }^{87 m} \mathrm{Sr}$ & $1 / 2-$ & $2.81 \mathrm{~h}$ & 388.53 & ० & ○ & ○ & $\circ$ & $\circ$ & $\circ$ & $\circ$ & ○ & ○ & ○ & ○ & $\circ$ & $\circ$ & ० & ○ & $\circ$ & ○ & ○ & ○ & ○ & ○ & ○ \\
\hline${ }^{88} \mathrm{Sr}$ & $0+$ & $82.58 \%$ & & o & $\circ$ & ○ & $\circ$ & ○ & ○ & $\circ$ & $\circ$ & $\circ$ & ○ & ○ & $\circ$ & ○ & ○ & ○ & ○ & ○ & ○ & $\circ$ & ○ & ○ & ○ \\
\hline${ }^{89} \mathrm{Sr}$ & $5 / 2+$ & $50.53 \mathrm{~d}$ & & o & o & o & $\circ$ & $\circ$ & $\circ$ & $\circ$ & $\circ$ & $\circ$ & ○ & ○ & $\circ$ & ○ & $\circ$ & ○ & $\circ$ & $\circ$ & ○ & ○ & ○ & ० & ○ \\
\hline${ }^{90} \mathrm{Sr}$ & $0+$ & $28.79 \mathrm{y}$ & & o & 0 & o & o & ० & ○ & o & ○ & ○ & ○ & ० & ○ & o & o & o & ○ & ○ & ○ & ○ & ○ & o & ○ \\
\hline${ }^{91} \mathrm{Sr}$ & $5 / 2+$ & $9.63 \mathrm{~h}$ & & $\circ$ & ० & ○ & ○ & ○ & ○ & ० & ○ & ○ & ○ & $\circ$ & $\circ$ & ○ & ○ & ○ & ○ & $\circ$ & ○ & ○ & o & ० & ○ \\
\hline${ }^{92} \mathrm{Sr}$ & $0+$ & $2.71 \mathrm{~h}$ & & ० & ○ & ० & $\circ$ & ० & $\circ$ & $\circ$ & $\circ$ & $\circ$ & $\circ$ & ○ & $\circ$ & $\circ$ & $\circ$ & $\circ$ & $\circ$ & $\circ$ & $\circ$ & $\circ$ & $\circ$ & $\circ$ & ○ \\
\hline
\end{tabular}

$+\infty$

\begin{tabular}{|c|c|c|c|c|c|c|}
\hline${ }^{84} g \mathrm{Y}$ & $1+$ & $4.6 \mathrm{~s}$ & & 0 & 0 & 0 \\
\hline${ }^{84 m} \mathrm{Y}$ & $5-$ & $39.5 \mathrm{~m}$ & $0.0+$ & ० & 0 & o \\
\hline${ }^{85} g \mathrm{Y}$ & $1 / 2$ & $2.68 \mathrm{~h}$ & & ○ & 0 & o \\
\hline${ }^{85 m} \mathrm{Y}$ & $9 / 2+$ & $4.86 \mathrm{~h}$ & 29.80 & ○ & ० & ○ \\
\hline${ }^{86} g \mathrm{Y}$ & 4- & $14.74 \mathrm{~h}$ & & $\bullet$ & ० & $\bullet$ \\
\hline${ }^{86 m} \mathrm{Y}$ & $8+$ & $48 \mathrm{~m}$ & 218.30 & ○ & ० & ○ \\
\hline${ }^{87} g \mathrm{Y}$ & $1 / 2-$ & $79.8 \mathrm{~h}$ & & $\bullet$ & $\bullet$ & $\bullet$ \\
\hline${ }^{87 m} \mathrm{Y}$ & $9 / 2+$ & $13.37 \mathrm{~h}$ & 380.79 & $\bullet$ & $\bullet$ & • \\
\hline${ }^{88}{ }^{g} \mathrm{Y}$ & 4- & $106.65 \mathrm{~d}$ & & $\bullet$ & $\bullet$ & • \\
\hline${ }^{88 m 1} \mathrm{Y}$ & $1+$ & $300 \mu \mathrm{s}$ & 392.86 & $\bullet$ & • & $\bullet$ \\
\hline${ }^{88 m 2} \mathrm{Y}$ & $8+$ & $13.9 \mathrm{~ms}$ & 674.55 & $\bullet$ & • & $\bullet$ \\
\hline${ }^{89}{ }^{g} \mathrm{Y}$ & $1 / 2-$ & $100 \%$ & & $\bullet$ & $\bullet$ & $\bullet$ \\
\hline${ }^{89 m} \mathrm{Y}$ & $9 / 2+$ & $15.28 \mathrm{~s}$ & 908.96 & $\bullet$ & $\bullet$ & • \\
\hline${ }^{90} \mathrm{Y}$ & $2-$ & $64.00 \mathrm{~h}$ & & $\bullet$ & o & $\bullet$ \\
\hline${ }^{90 m} \mathrm{Y}$ & $7+$ & $3.19 \mathrm{~h}$ & 682.03 & ० & ० & ○ \\
\hline${ }^{91} g \mathrm{Y}$ & $1 / 2-$ & $58.51 \mathrm{~d}$ & & $\bullet$ & o & $\bullet$ \\
\hline
\end{tabular}

Continued on next page... 
Table 6: (continued)

\begin{tabular}{|c|c|c|c|c|c|c|c|c|c|c|c|c|c|c|c|c|c|c|c|c|c|c|c|c|c|}
\hline Target & $J^{\pi}$ & $t_{1 / 2}$ & Energy & $\stackrel{2}{\approx}$ & $\approx$ & 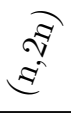 & 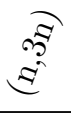 & 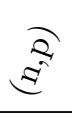 & 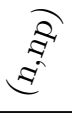 & $\underset{g}{g}$ & $\underset{\Xi}{\stackrel{g}{g}}$ & $\underset{\nabla}{\approx}$ & $\stackrel{F}{E}$ & $\approx$ & $\stackrel{\vartheta}{\vec{\nabla}}$ & $\widehat{\hat{2}}$ & $\underset{8}{\stackrel{\Xi}{8}}$ & $\stackrel{2}{2}$ & 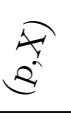 & $\underset{\nexists}{\nexists}$ & $\underset{\widetilde{\nabla}}{\stackrel{\nabla}{\sigma}}$ & থ્જી & $\stackrel{2}{2}$ & $\underset{i}{i}$ & $\underset{0}{\overparen{f}}$ \\
\hline${ }^{91 m} \mathrm{Y}$ & $9 / 2+$ & $49.71 \mathrm{~m}$ & 555.58 & ○ & $\circ$ & ○ & ○ & ○ & ○ & ० & ○ & ○ & ○ & $\circ$ & $\circ$ & ○ & ○ & ० & ○ & ○ & ○ & $\circ$ & $\circ$ & ○ & ○ \\
\hline${ }^{92} \mathrm{Y}$ & $2-$ & $3.54 \mathrm{~h}$ & & • & ० & $\bullet$ & $\bullet$ & ० & ० & o & ० & o & $\bullet$ & ० & ० & ० & ० & ० & ० & o & o & ० & ० & ० & $\circ$ \\
\hline${ }^{93} g \mathrm{Y}$ & $1 / 2-$ & $10.18 \mathrm{~h}$ & & ० & ० & ० & ○ & ० & ० & ○ & ० & $\circ$ & ० & ० & ० & ○ & ० & ० & ० & ○ & ○ & $\circ$ & ○ & ० & ○ \\
\hline${ }^{93 m} \mathrm{Y}$ & $7 / 2+$ & $0.82 \mathrm{~s}$ & 758.72 & ० & $\circ$ & ० & ○ & ○ & $\circ$ & ० & ○ & $\circ$ & ० & ० & $\circ$ & ० & ० & ० & ० & ० & ० & $\circ$ & $\circ$ & ० & ० \\
\hline
\end{tabular}

\begin{tabular}{|c|c|c|c|c|c|c|c|c|c|c|c|c|c|c|c|c|c|c|c|c|c|c|c|c|c|}
\hline${ }^{86} \mathrm{Zr}$ & $0+$ & $16.5 \mathrm{~h}$ & & 0 & 0 & 0 & & 0 & ० & o & 0 & 0 & 0 & 0 & 0 & 0 & o & o & o & 0 & 0 & & o & ○ & ○ \\
\hline${ }^{87 g} \mathrm{Zr}$ & $9 / 2+$ & $1.68 \mathrm{~h}$ & & • & ○ & • & & • & ○ & ○ & ० & ○ & ○ & ○ & ○ & $\circ$ & ○ & $\circ$ & $\circ$ & ○ & ○ & & ○ & ○ & ○ \\
\hline${ }^{87 m} \mathrm{Zr}$ & $1 / 2$ & $14 \mathrm{~s}$ & 335.73 & $\circ$ & $\circ$ & $\circ$ & & $\circ$ & $\circ$ & $\circ$ & ○ & $\circ$ & $\circ$ & $\circ$ & $\circ$ & $\circ$ & ○ & $\circ$ & $\circ$ & $\circ$ & ○ & & ○ & ० & ○ \\
\hline${ }^{88} \mathrm{Zr}$ & $0+$ & $83.4 \mathrm{~d}$ & & • & ○ & • & & $\bullet$ & • & $\circ$ & $\circ$ & ○ & ○ & ○ & ○ & ○ & ○ & $\circ$ & $\circ$ & ○ & $\circ$ & & ○ & ○ & ○ \\
\hline${ }^{89} \mathrm{Z} \mathrm{Zr}$ & $9 / 2+$ & $78.41 \mathrm{~h}$ & & • & $\bullet$ & • & & • & $\bullet$ & $\circ$ & o & ○ & ○ & $\circ$ & ○ & $\circ$ & ○ & $\circ$ & $\circ$ & ○ & ○ & ○ & ○ & ○ & ○ \\
\hline${ }^{89 m} \mathrm{Zr}$ & $1 / 2-$ & $4.161 \mathrm{~m}$ & 587.84 & • & $\bullet$ & $\bullet$ & & • & $\bullet$ & $\circ$ & o & ○ & ○ & ○ & ○ & $\circ$ & ○ & $\circ$ & $\circ$ & $\circ$ & ○ & $\circ$ & $\circ$ & ○ & ○ \\
\hline${ }^{90} \mathrm{~g} \mathrm{Zr}$ & $0+$ & $51.45 \%$ & & • & $\bullet$ & • & & • & $\bullet$ & $\circ$ & o & $\circ$ & • & ○ & ○ & $\circ$ & ○ & $\circ$ & $\circ$ & ○ & ○ & $\circ$ & ○ & ○ & ○ \\
\hline${ }^{90 m^{1}} \mathrm{Zr}$ & $0+$ & $61.3 \mathrm{~ns}$ & 1760.71 & $\bullet$ & $\bullet$ & $\bullet$ & o & $\bullet$ & $\bullet$ & $\circ$ & ○ & ○ & $\bullet$ & ○ & ○ & $\circ$ & o & $\circ$ & $\circ$ & ○ & ○ & ○ & ○ & ○ & ○ \\
\hline $90 \mathrm{~m}^{2} \mathrm{Zr}$ & $2+$ & 88.4 fs & 2186.27 & $\bullet$ & $\bullet$ & $\bullet$ & $\circ$ & $\bullet$ & $\bullet$ & $\circ$ & ० & $\circ$ & $\bullet$ & ○ & ○ & ○ & $\circ$ & $\circ$ & $\circ$ & $\circ$ & ○ & $\circ$ & $\circ$ & ○ & ○ \\
\hline $90 m^{3} \mathrm{Zr}$ & $5-$ & $809.2 \mathrm{~ms}$ & 2319.00 & $\bullet$ & $\bullet$ & $\bullet$ & ○ & $\bullet$ & $\bullet$ & $\circ$ & ० & ○ & $\bullet$ & ○ & $\circ$ & $\circ$ & ○ & $\circ$ & $\circ$ & $\circ$ & ○ & ○ & $\circ$ & ○ & ○ \\
\hline${ }^{91} \mathrm{Z} \mathrm{Zr}$ & $5 / 2+$ & $11.22 \%$ & & ○ & ○ & $\bullet$ & ○ & • & ○ & $\circ$ & ○ & ○ & ○ & ○ & ○ & ○ & ○ & $\circ$ & $\circ$ & ○ & ○ & ○ & ○ & ○ & ○ \\
\hline${ }^{91 m} \mathrm{Zr}$ & $21 / 2+$ & $4.35 \mu \mathrm{s}$ & 3167.30 & ○ & ○ & $\circ$ & ० & $\circ$ & $\circ$ & ○ & ० & ○ & ○ & ○ & ○ & ० & ० & $\circ$ & ० & ○ & ○ & ○ & ○ & ○ & ० \\
\hline${ }^{92} \mathrm{Zr}$ & $0+$ & $17.15 \%$ & & ○ & ○ & • & ○ & ○ & ○ & $\circ$ & ○ & ○ & ○ & ○ & ○ & $\circ$ & ○ & $\circ$ & $\circ$ & ○ & ○ & ○ & ○ & ○ & ○ \\
\hline${ }^{93} \mathrm{Zr}$ & $5 / 2+$ & $1.53 \mathrm{My}$ & & $\circ$ & $\circ$ & $\circ$ & ○ & $\circ$ & $\circ$ & $\circ$ & $\circ$ & $\circ$ & $\circ$ & $\circ$ & $\circ$ & $\circ$ & ○ & $\circ$ & $\circ$ & $\circ$ & ○ & $\circ$ & ○ & ० & ○ \\
\hline${ }^{94} \mathrm{Zr}$ & $0+$ & $17.38 \%$ & & ○ & ○ & ○ & o & $\circ$ & ○ & $\circ$ & ○ & ○ & ○ & ○ & ○ & ○ & ○ & $\circ$ & ○ & $\circ$ & $\circ$ & $\circ$ & ○ & ○ & ० \\
\hline${ }^{95} \mathrm{Zr}$ & $5 / 2+$ & $64.02 \mathrm{~d}$ & & $\circ$ & ○ & $\circ$ & ○ & ○ & $\circ$ & ○ & o & ○ & $\circ$ & $\circ$ & $\circ$ & $\circ$ & ○ & ○ & $\circ$ & $\circ$ & $\circ$ & ○ & ○ & ○ & ○ \\
\hline${ }^{96} \mathrm{Zr}$ & $0+$ & $2.80 \%$ & & ○ & ○ & ○ & $\circ$ & $\circ$ & ○ & ○ & ○ & ○ & ○ & ○ & ○ & ० & o & $\circ$ & $\circ$ & ○ & ○ & ○ & ○ & ○ & ○ \\
\hline${ }^{87} g \mathrm{Nb}$ & $9 / 2+$ & $2.6 \mathrm{~m}$ & & o & o & 0 & & 0 & o & o & 0 & $\circ$ & $\circ$ & o & 0 & 0 & 0 & 0 & o & $\circ$ & $\circ$ & & $\circ$ & o & $\circ$ \\
\hline${ }^{87 m} \mathrm{Nb}$ & $1 / 2-$ & $3.7 \mathrm{~m}$ & $0.0+$ & ○ & ○ & ○ & & $\circ$ & ○ & $\circ$ & o & ○ & ○ & ○ & ○ & $\circ$ & ० & $\circ$ & $\circ$ & ○ & ○ & & ○ & $\circ$ & ○ \\
\hline${ }^{88} g \mathrm{Nb}$ & $8+$ & $14.5 \mathrm{~m}$ & & ○ & ○ & ○ & & $\circ$ & $\circ$ & ○ & ० & ○ & ○ & ○ & ○ & ० & o & $\circ$ & $\circ$ & ○ & o & ○ & ○ & ○ & ० \\
\hline${ }^{88 m} \mathrm{Nb}$ & 4- & $7.8 \mathrm{~m}$ & $0.0+$ & $\circ$ & ○ & $\circ$ & & ○ & $\circ$ & $\circ$ & o & $\circ$ & $\circ$ & $\circ$ & $\circ$ & ○ & ○ & $\circ$ & ○ & $\circ$ & $\circ$ & $\circ$ & $\circ$ & ○ & ○ \\
\hline${ }^{89} g \mathrm{Nb}$ & $9 / 2+$ & $2.03 \mathrm{~h}$ & & $\circ$ & $\circ$ & ○ & & ○ & $\circ$ & $\circ$ & ○ & $\circ$ & ○ & $\circ$ & $\circ$ & ○ & ○ & $\circ$ & $\circ$ & $\circ$ & ○ & $\circ$ & $\circ$ & ○ & ○ \\
\hline${ }^{89 m} \mathrm{Nb}$ & $1 / 2$ & $66 \mathrm{~m}$ & $0.0+$ & ○ & $\circ$ & ○ & & $\circ$ & $\circ$ & $\circ$ & $\circ$ & $\circ$ & ○ & $\circ$ & ○ & $\circ$ & $\circ$ & $\circ$ & $\circ$ & $\circ$ & ○ & $\circ$ & $\circ$ & ○ & ○ \\
\hline
\end{tabular}

Continued on next page... 
Table 6: (continued)

\begin{tabular}{|c|c|c|c|c|c|c|c|c|c|c|c|c|c|c|c|c|c|c|c|c|c|c|c|c|c|}
\hline Target & $J^{\pi}$ & $t_{1 / 2}$ & Energy & $\underset{\Xi}{\stackrel{2}{2}}$ & $\underset{\Xi}{\Xi}$ & $\underset{\approx}{\approx}$ & 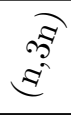 & $\stackrel{શ}{\nexists}$ & $\underset{\Xi}{\stackrel{\approx}{\approx}}$ & $\stackrel{\partial}{\nexists}$ & $\underset{\Xi}{\Xi}$ & $\underset{\nexists}{\nexists}$ & $\stackrel{\nexists}{E}$ & $\underset{8}{8}$ & $\begin{array}{l}\stackrel{\vartheta}{\vartheta} \\
\stackrel{8}{ }\end{array}$ & $\widehat{2}$ & $\stackrel{\Xi}{\Omega}$ & $\stackrel{2}{2}$ & $\vec{\Omega}$ & $\underset{\Xi}{\nexists}$ & $\underset{\nabla}{\vec{\nabla}}$ & 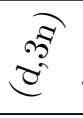 & $\stackrel{2}{2}$ & $\underset{0}{0}$ & 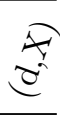 \\
\hline${ }^{90} g \mathrm{Nb}$ & $8+$ & $14.60 \mathrm{~h}$ & & $\circ$ & ○ & $\circ$ & & ○ & ○ & o & $\circ$ & ○ & $\circ$ & $\circ$ & ○ & ○ & ○ & ○ & ○ & $\circ$ & $\circ$ & o & ○ & 0 & $\circ$ \\
\hline${ }^{90 m 1} \mathrm{Nb}$ & $6+$ & $63 \mu \mathrm{s}$ & 122.37 & $\circ$ & $\circ$ & $\circ$ & & $\circ$ & o & o & ○ & $\circ$ & $\circ$ & $\circ$ & ○ & ○ & $\circ$ & $\circ$ & $\circ$ & ○ & ○ & $\circ$ & $\circ$ & $\circ$ & $\circ$ \\
\hline $90 m^{2} \mathrm{Nb}$ & 4- & $18.81 \mathrm{~s}$ & 124.67 & ○ & ○ & ○ & & o & o & o & ○ & ○ & ○ & ○ & ○ & ○ & ○ & o & ○ & ○ & ○ & ○ & ○ & ○ & ○ \\
\hline $90 m 3 \mathrm{Nb}$ & $1+$ & $6.19 \mathrm{~ms}$ & 382.01 & $\circ$ & $\circ$ & $\circ$ & & $\circ$ & $\circ$ & $\circ$ & $\circ$ & $\circ$ & $\circ$ & $\circ$ & $\circ$ & $\circ$ & $\circ$ & $\circ$ & $\circ$ & $\circ$ & $\circ$ & $\circ$ & ○ & $\circ$ & $\circ$ \\
\hline${ }^{91} g \mathrm{Nb}$ & $9 / 2+$ & $680 \mathrm{y}$ & & $\circ$ & $\circ$ & $\circ$ & & $\circ$ & $\circ$ & $\circ$ & $\circ$ & $\circ$ & $\circ$ & $\circ$ & $\circ$ & $\circ$ & $\circ$ & $\circ$ & $\circ$ & ० & $\circ$ & $\circ$ & ○ & $\circ$ & $\circ$ \\
\hline${ }^{91 m} 1 \mathrm{Nb}$ & $1 / 2$ & $60.86 \mathrm{~d}$ & 104.49 & $\circ$ & $\circ$ & $\circ$ & & ० & ○ & ० & ○ & ○ & ○ & $\circ$ & ○ & ○ & ○ & $\circ$ & ○ & ○ & ○ & ○ & ○ & $\circ$ & $\circ$ \\
\hline $91 m 2 \mathrm{Nb}$ & $17 / 2-$ & $3.76 \mu \mathrm{s}$ & 2034.36 & $\circ$ & $\circ$ & $\circ$ & & ○ & $\circ$ & $\circ$ & ○ & $\circ$ & $\circ$ & $\circ$ & $\circ$ & ○ & ○ & $\circ$ & $\circ$ & $\circ$ & ○ & $\circ$ & ○ & $\circ$ & $\circ$ \\
\hline${ }^{92 g} \mathrm{Nb}$ & $7+$ & 34.7 My & & ○ & $\bullet$ & ○ & ० & ○ & ○ & ○ & $\circ$ & ○ & $\bullet$ & ○ & ○ & ○ & ○ & ○ & ○ & ○ & $\circ$ & $\circ$ & ○ & $\circ$ & ○ \\
\hline${ }^{92 m 1} \mathrm{Nb}$ & $2+$ & $10.15 \mathrm{~d}$ & 135.50 & ○ & $\bullet$ & ○ & ○ & ○ & ○ & ० & $\circ$ & ○ & $\bullet$ & ○ & ○ & ○ & ○ & ○ & ○ & ○ & $\circ$ & ○ & ○ & $\circ$ & ○ \\
\hline $92 m^{2} \mathrm{Nb}$ & $2-$ & $5.9 \mu \mathrm{s}$ & 225.70 & ○ & $\bullet$ & ○ & ○ & ○ & $\circ$ & $\circ$ & $\circ$ & $\circ$ & $\bullet$ & $\circ$ & ○ & ○ & ○ & ○ & ○ & ○ & ○ & ○ & ○ & ○ & ○ \\
\hline${ }^{93} g \mathrm{Nb}$ & $9 / 2+$ & $100 \%$ & & ○ & $\bullet$ & $\bullet$ & ○ & o & ○ & o & ○ & $\circ$ & $\bullet$ & ○ & $\circ$ & ○ & $\circ$ & ○ & ○ & ○ & ○ & $\circ$ & $\circ$ & ○ & ○ \\
\hline${ }^{93 m} \mathrm{Nb}$ & $1 / 2$ & $16.13 \mathrm{y}$ & 30.82 & $\circ$ & $\bullet$ & $\bullet$ & $\circ$ & ० & ○ & ० & $\circ$ & $\circ$ & $\bullet$ & $\circ$ & $\circ$ & ○ & $\circ$ & $\circ$ & ○ & $\circ$ & $\circ$ & $\circ$ & $\circ$ & $\circ$ & $\circ$ \\
\hline${ }^{94} g \mathrm{Nb}$ & $6+$ & $2030 \mathrm{y}$ & & $\circ$ & $\circ$ & $\circ$ & ○ & o & ○ & $\circ$ & ○ & $\circ$ & $\circ$ & $\circ$ & ○ & ○ & $\circ$ & $\circ$ & ○ & ○ & ○ & $\circ$ & $\circ$ & $\circ$ & $\circ$ \\
\hline${ }^{94 m} \mathrm{Nb}$ & $3+$ & $6.263 \mathrm{~m}$ & 40.90 & $\circ$ & $\circ$ & $\circ$ & ○ & ० & $\circ$ & ○ & ○ & ○ & ○ & ○ & ○ & ○ & $\circ$ & $\circ$ & ○ & ○ & ○ & ○ & $\circ$ & $\circ$ & $\circ$ \\
\hline${ }^{95} g \mathrm{Nb}$ & $9 / 2+$ & $34.997 \mathrm{~d}$ & & $\circ$ & $\circ$ & $\circ$ & $\circ$ & $\circ$ & $\circ$ & $\circ$ & $\circ$ & $\circ$ & $\circ$ & $\circ$ & $\circ$ & $\circ$ & $\circ$ & $\circ$ & $\circ$ & $\circ$ & $\circ$ & $\circ$ & ○ & $\circ$ & $\circ$ \\
\hline${ }^{95 m} \mathrm{Nb}$ & $1 / 2$ & $86.6 \mathrm{~h}$ & 235.68 & $\circ$ & $\circ$ & $\circ$ & $\circ$ & $\circ$ & $\circ$ & $\circ$ & $\circ$ & $\circ$ & $\circ$ & ○ & $\circ$ & ○ & ○ & $\circ$ & ○ & $\circ$ & $\circ$ & $\circ$ & $\circ$ & $\circ$ & $\circ$ \\
\hline${ }^{96} \mathrm{Nb}$ & $6+$ & $23.35 \mathrm{~h}$ & & $\circ$ & $\circ$ & $\circ$ & ○ & ० & ○ & $\circ$ & ○ & ○ & ○ & $\circ$ & o & ○ & ○ & $\circ$ & ○ & ○ & ○ & ○ & ○ & $\circ$ & $\circ$ \\
\hline${ }^{97} g \mathrm{Nb}$ & $9 / 2+$ & $72.1 \mathrm{~m}$ & & $\circ$ & $\circ$ & $\circ$ & $\circ$ & ० & ○ & ० & ○ & $\circ$ & ○ & $\circ$ & o & ○ & $\circ$ & $\circ$ & ○ & ○ & ○ & $\circ$ & ○ & $\circ$ & $\circ$ \\
\hline${ }^{97 m} \mathrm{Nb}$ & $1 / 2$ & $58.7 \mathrm{~s}$ & 743.35 & ○ & $\circ$ & $\circ$ & ○ & ० & ○ & ० & ○ & ○ & ○ & ○ & ○ & ○ & ○ & $\circ$ & ○ & ○ & ○ & ○ & ○ & $\circ$ & ○ \\
\hline${ }^{98} g \mathrm{Nb}$ & $1+$ & $2.86 \mathrm{~s}$ & & ○ & ○ & ○ & ○ & o & ○ & o & ○ & ○ & ○ & $\circ$ & ○ & ○ & ○ & ○ & ○ & ○ & ○ & ○ & ○ & ○ & ○ \\
\hline${ }^{98 m} \mathrm{Nb}$ & $5+$ & $51.3 \mathrm{~m}$ & 84.00 & $\circ$ & $\circ$ & $\circ$ & $\circ$ & ○ & $\circ$ & $\circ$ & $\circ$ & $\circ$ & $\circ$ & $\circ$ & $\circ$ & $\circ$ & $\circ$ & ○ & $\circ$ & $\circ$ & $\circ$ & $\circ$ & $\circ$ & $\circ$ & $\circ$ \\
\hline${ }^{99} g \mathrm{Nb}$ & $9 / 2+$ & $15.0 \mathrm{~s}$ & & ○ & ○ & ○ & ○ & o & ○ & ○ & ○ & ○ & ○ & ○ & ○ & ○ & ○ & ○ & ○ & ○ & ○ & ○ & ○ & ○ & ○ \\
\hline${ }^{99 m} \mathrm{Nb}$ & $1 / 2$ & $2.6 \mathrm{~m}$ & 365.29 & $\circ$ & $\circ$ & $\circ$ & $\circ$ & ० & $\circ$ & $\circ$ & $\circ$ & $\circ$ & $\circ$ & $\circ$ & $\circ$ & $\circ$ & $\circ$ & $\circ$ & $\circ$ & $\circ$ & $\circ$ & $\circ$ & $\circ$ & $\circ$ & $\circ$ \\
\hline${ }^{100} \mathrm{Nb}$ & $1+$ & $1.5 \mathrm{~s}$ & & ○ & $\circ$ & $\circ$ & $\circ$ & ० & $\circ$ & $\circ$ & $\circ$ & $\circ$ & $\circ$ & $\circ$ & $\circ$ & ○ & $\circ$ & $\circ$ & $\circ$ & $\circ$ & $\circ$ & $\circ$ & $\circ$ & $\circ$ & ○ \\
\hline${ }^{90} g \mathrm{Mo}$ & $0+$ & $5.56 \mathrm{~h}$ & & $\circ$ & $\circ$ & $\circ$ & & $\circ$ & $\circ$ & $\circ$ & $\circ$ & $\circ$ & $\circ$ & $\circ$ & & $\circ$ & $\circ$ & $\circ$ & $\circ$ & $\circ$ & $\circ$ & & ○ & $\circ$ & $\circ$ \\
\hline${ }^{90 m} \mathrm{Mo}$ & $8+$ & $1.12 \mu \mathrm{s}$ & 2874.73 & $\circ$ & $\circ$ & $\circ$ & & $\circ$ & $\circ$ & $\circ$ & $\circ$ & $\circ$ & $\circ$ & $\circ$ & $\circ$ & $\circ$ & $\circ$ & $\circ$ & $\circ$ & $\circ$ & $\circ$ & & $\circ$ & $\circ$ & $\circ$ \\
\hline${ }^{91 g} \mathrm{Mo}$ & $9 / 2+$ & $15.49 \mathrm{~m}$ & & $\circ$ & $\bullet$ & $\circ$ & & $\circ$ & $\circ$ & • & $\circ$ & $\circ$ & $\bullet$ & $\circ$ & $\circ$ & $\circ$ & $\circ$ & $\circ$ & $\circ$ & $\circ$ & $\circ$ & & $\circ$ & $\circ$ & $\circ$ \\
\hline${ }^{91 m} \mathrm{Mo}$ & $1 / 2$ & $64.6 \mathrm{~s}$ & 653.01 & $\circ$ & $\bullet$ & ○ & & $\circ$ & $\circ$ & $\bullet$ & $\circ$ & $\circ$ & $\bullet$ & $\circ$ & $\circ$ & $\circ$ & $\circ$ & $\circ$ & $\circ$ & $\circ$ & $\circ$ & & ○ & $\circ$ & $\circ$ \\
\hline${ }^{92} \mathrm{Mo}$ & $0+$ & $14.84 \%$ & & $\circ$ & ○ & $\bullet$ & & $\bullet$ & ० & $\bullet$ & $\bullet$ & ○ & $\bullet$ & ० & ○ & $\circ$ & $\circ$ & ० & ○ & ○ & ○ & & ○ & $\circ$ & ○ \\
\hline
\end{tabular}

Continued on next page... 
Table 6: (continued)

\begin{tabular}{|c|c|c|c|c|c|c|c|c|c|c|c|c|c|c|c|c|c|c|c|c|c|c|c|c|c|}
\hline Target & $J^{\pi}$ & $t_{1 / 2}$ & Energy & ते & $\approx$ & $\underset{\nexists}{\approx}$ & 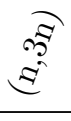 & $\stackrel{2}{2}$ & $\underset{\Xi}{\stackrel{\Xi}{\Xi}}$ & $\underset{\Xi}{\sigma}$ & $\underset{\Xi}{\stackrel{\Xi}{\Xi}}$ & $\underset{\approx}{\approx}$ & $\stackrel{E}{E}$ & $\stackrel{9}{8}$ & $\stackrel{\vartheta}{\stackrel{\nabla}{\Omega}}$ & $\widehat{2}$ & $\underset{\Omega}{\stackrel{\Xi}{g}}$ & $\stackrel{2}{2}$ & $\vec{Q}$ & $\underset{\Xi}{\overparen{Z}}$ & $\underset{\nabla}{\stackrel{\nabla}{\sigma}}$ & i্ & 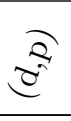 & $\underset{j}{\partial}$ & $\underset{t}{\nexists t}$ \\
\hline${ }^{93} \mathrm{Mo}$ & $5 / 2+$ & $4000 \mathrm{y}$ & & $\circ$ & ○ & ○ & & o & o & o & ○ & ○ & ○ & $\circ$ & ○ & $\circ$ & o & o & o & ○ & ○ & ○ & ○ & o & ○ \\
\hline${ }^{93 m} \mathrm{Mo}$ & $21 / 2+$ & $6.85 \mathrm{~h}$ & 2424.89 & $\circ$ & $\circ$ & $\circ$ & o & $\circ$ & ० & ० & ○ & $\circ$ & $\circ$ & $\circ$ & $\circ$ & $\circ$ & ० & ० & ० & ○ & $\circ$ & $\circ$ & $\circ$ & ० & o \\
\hline${ }^{94} \mathrm{Mo}$ & $0+$ & $9.25 \%$ & & ○ & ○ & $\circ$ & ० & ० & ○ & ○ & $\circ$ & $\circ$ & $\circ$ & ○ & ○ & ○ & ० & ० & $\circ$ & ○ & $\circ$ & $\circ$ & $\circ$ & ० & o \\
\hline${ }^{95} \mathrm{Mo}$ & $5 / 2+$ & $15.92 \%$ & & ○ & ○ & ○ & ० & ○ & ○ & o & ○ & o & ○ & ○ & ○ & ○ & ० & o & ० & ○ & ○ & ○ & ○ & ○ & $\circ$ \\
\hline${ }^{96} \mathrm{Mo}$ & $0+$ & $16.68 \%$ & & ○ & ० & ○ & ० & ○ & ○ & o & ○ & o & ○ & ○ & ० & ○ & ० & ० & ० & ○ & ○ & o & ○ & ० & o \\
\hline${ }^{97} \mathrm{Mo}$ & $5 / 2+$ & $9.55 \%$ & & o & o & o & o & o & o & o & o & o & o & o & o & o & o & o & o & ○ & ○ & o & o & o & o \\
\hline${ }^{98} \mathrm{Mo}$ & $0+$ & $24.13 \%$ & & ○ & ○ & $\circ$ & $\circ$ & ○ & $\circ$ & ○ & ○ & ○ & ○ & ○ & ○ & $\circ$ & $\circ$ & ○ & ○ & ○ & ○ & o & ○ & ○ & o \\
\hline${ }^{99} \mathrm{Mo}$ & $1 / 2+$ & $65.94 \mathrm{~h}$ & & $\circ$ & ○ & $\circ$ & $\circ$ & $\circ$ & $\circ$ & ○ & $\circ$ & $\circ$ & $\circ$ & $\circ$ & ○ & $\circ$ & $\circ$ & ० & ○ & ○ & $\circ$ & ० & $\circ$ & ० & $\circ$ \\
\hline${ }^{99 m} \mathrm{Mo}$ & $5 / 2+$ & $15.5 \mu \mathrm{s}$ & 97.79 & ○ & ○ & ○ & ० & $\circ$ & $\circ$ & ० & ○ & ○ & ○ & ○ & ○ & $\circ$ & ० & ० & ० & ○ & ○ & ० & ○ & ० & ○ \\
\hline${ }^{100} \mathrm{Mo}$ & $0+$ & $9.63 \%$ & & ○ & ○ & ○ & ० & $\circ$ & $\circ$ & ○ & $\circ$ & $\circ$ & $\circ$ & ○ & ○ & $\circ$ & ० & $\circ$ & $\circ$ & ○ & ○ & ○ & $\circ$ & ○ & o \\
\hline${ }^{101} \mathrm{Mo}$ & $1 / 2+$ & $14.61 \mathrm{~m}$ & & ○ & ○ & $\circ$ & $\circ$ & $\circ$ & $\circ$ & ○ & $\circ$ & $\circ$ & $\circ$ & ○ & ○ & $\circ$ & $\circ$ & $\circ$ & $\circ$ & $\circ$ & $\circ$ & ○ & $\circ$ & ○ & o \\
\hline
\end{tabular}

\begin{tabular}{|c|c|c|c|c|c|c|c|c|c|c|c|c|c|c|c|c|c|c|c|c|c|c|c|c|}
\hline${ }^{91} g \mathrm{Tc}$ & $9 / 2+$ & $3.14 \mathrm{~m}$ & & 0 & 0 & 0 & & 0 & 0 & 0 & 0 & o & 0 & 0 & 0 & 0 & 0 & 0 & 0 & 0 & 0 & & 0 & 0 \\
\hline${ }^{91 m} \mathrm{Tc}$ & $1 / 2-$ & $3.3 \mathrm{~m}$ & 350.00 & ○ & ० & $\circ$ & & $\circ$ & ○ & $\circ$ & ० & ○ & ○ & ○ & $\circ$ & ○ & $\circ$ & o & ० & ० & ० & & ० & o \\
\hline${ }^{92} \mathrm{Tc}$ & $8+$ & $4.23 \mathrm{~m}$ & & ० & ० & 0 & & $\circ$ & 0 & 0 & 0 & ○ & 0 & 0 & $\circ$ & 0 & 0 & 0 & ○ & ○ & ○ & & ० & ० \\
\hline${ }^{92 m} \mathrm{Tc}$ & $4+$ & $1.03 \mu \mathrm{s}$ & 270.15 & $\circ$ & ० & ○ & & o & ० & ○ & 0 & ○ & ○ & $\circ$ & 0 & o & $\circ$ & 0 & $\circ$ & ○ & ○ & & ○ & ० \\
\hline${ }^{93} g \mathrm{Tc}$ & $9 / 2+$ & $2.75 \mathrm{~h}$ & & ० & ० & ० & & $\circ$ & ० & ○ & ० & ० & ० & ० & ० & ○ & ० & o & $\circ$ & ○ & ○ & & ० & ० \\
\hline${ }^{93} m^{1} \mathrm{Tc}$ & $1 / 2-$ & $43.5 \mathrm{~m}$ & 391.84 & ० & o & o & & ० & o & ० & ० & ○ & ० & ० & ० & ० & ० & 0 & ० & ० & ० & o & o & o \\
\hline${ }^{93} m^{2} \mathrm{Tc}$ & $17 / 2-$ & $10.2 \mu \mathrm{s}$ & 2185.16 & ○ & ० & ○ & & $\circ$ & ○ & ० & ० & ○ & ० & ○ & ० & $\circ$ & $\circ$ & ○ & $\circ$ & $\circ$ & ○ & $\circ$ & ० & ० \\
\hline${ }^{94} \mathrm{Tc}$ & $7+$ & $293 \mathrm{~m}$ & & ○ & ० & ○ & & $\circ$ & ○ & $\circ$ & ○ & ○ & $\circ$ & ० & $\circ$ & ○ & ○ & ○ & ○ & ○ & ○ & ○ & ○ & ० \\
\hline${ }^{94 m} \mathrm{Tc}$ & $2+$ & $52.0 \mathrm{~m}$ & 75.70 & ○ & o & ० & & ○ & o & $\circ$ & ○ & ○ & $\circ$ & ० & $\circ$ & ○ & ० & ० & ० & ० & ० & ○ & ० & ० \\
\hline${ }^{95} \mathrm{Tc}$ & $9 / 2+$ & $20.0 \mathrm{~h}$ & & ○ & o & ○ & 0 & ○ & ० & ○ & ○ & ० & ○ & ○ & ○ & ○ & ○ & ० & ○ & ○ & ० & ○ & ○ & o \\
\hline${ }^{95 m} \mathrm{Tc}$ & $1 / 2-$ & $61 \mathrm{~d}$ & 38.89 & $\circ$ & ○ & ○ & ० & ० & ○ & ○ & $\circ$ & ○ & ○ & ○ & ○ & ○ & $\circ$ & ० & $\circ$ & ○ & $\circ$ & ○ & ○ & O \\
\hline${ }^{96} g_{\mathrm{Tc}}$ & $7+$ & $4.28 \mathrm{~d}$ & & 0 & 0 & ○ & 0 & o & 0 & ○ & ○ & ○ & ○ & ○ & ○ & ○ & ○ & o & ○ & ○ & ○ & ○ & o & O \\
\hline${ }^{96 m} \mathrm{Tc}$ & $4+$ & $51.5 \mathrm{~m}$ & 34.28 & ○ & ○ & $\circ$ & $\circ$ & ० & ० & $\circ$ & ० & ○ & ○ & ○ & ० & ० & ० & o & ० & ० & ○ & ○ & ○ & O \\
\hline${ }^{97 g} \mathrm{Tc}$ & $9 / 2+$ & $4.21 \mathrm{My}$ & & ० & o & ० & $\circ$ & ○ & ० & $\circ$ & ० & ० & ० & ० & ० & ० & ० & o & ० & ० & ० & ० & o & o \\
\hline${ }^{97 m} \mathrm{Tc}$ & $1 / 2-$ & $91.4 \mathrm{~d}$ & 96.56 & $\circ$ & ० & $\circ$ & ० & $\circ$ & ○ & $\circ$ & $\circ$ & ○ & $\circ$ & $\circ$ & $\circ$ & $\circ$ & $\circ$ & ० & $\circ$ & ○ & ○ & $\circ$ & ० & 0 \\
\hline${ }^{98 g} \mathrm{Tc}$ & $6+$ & $4.2 \mathrm{My}$ & & ○ & ० & ० & ० & $\circ$ & ० & $\circ$ & $\circ$ & ○ & ○ & ० & ० & $\circ$ & $\circ$ & ० & ० & ० & ० & ० & ० & ० \\
\hline${ }^{98 m} \mathrm{Tc}$ & $2-$ & $14.7 \mu \mathrm{s}$ & 90.76 & ० & ० & ० & ० & ○ & $\circ$ & $\circ$ & ० & ○ & ○ & ० & ० & ० & o & ० & ० & ० & ० & $\circ$ & ○ & ० \\
\hline${ }^{99} \mathrm{Tc}$ & $9 / 2+$ & $0.2111 \mathrm{My}$ & & $\circ$ & ○ & $\circ$ & ○ & $\circ$ & ० & ○ & ० & ० & ० & ○ & ० & $\circ$ & $\circ$ & ० & ० & ० & ○ & ० & ○ & ० \\
\hline
\end{tabular}

Continued on next page... 
Table 6: (continued)

\begin{tabular}{|c|c|c|c|c|c|c|c|c|c|c|c|c|c|c|c|c|c|c|c|c|c|c|c|c|c|}
\hline Target & $J^{\pi}$ & $t_{1 / 2}$ & Energy & $\stackrel{2}{\approx}$ & $\nexists$ & $\underset{\approx}{\approx}$ & શ્ฐ & શ & 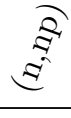 & $\underset{\Xi}{g}$ & $\underset{\Xi}{\stackrel{\Xi}{\Xi}}$ & $\underset{\approx}{\approx}$ & $\stackrel{\nexists}{\Xi}$ & $\stackrel{\nexists}{\Xi}$ & $\stackrel{\vec{\nabla}}{\stackrel{\nabla}{2}}$ & $\hat{2}$ & $\stackrel{8}{8}$ & $\stackrel{2}{2}$ & $\vec{Q}$ & $\underset{\nexists}{\nexists}$ & $\underset{\nabla}{\stackrel{\nabla}{\sigma}}$ & 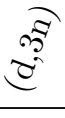 & হి & $\underset{\delta}{\delta}$ & 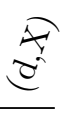 \\
\hline${ }^{99 m} \mathrm{Tc}$ & $1 / 2-$ & $6.01 \mathrm{~h}$ & 142.68 & o & 0 & 0 & $\circ$ & ○ & ○ & o & o & 0 & 0 & 0 & 0 & $\circ$ & $\circ$ & 0 & 0 & 0 & 0 & 0 & 0 & 0 & $\circ$ \\
\hline${ }^{100 g} \mathrm{Tc}$ & $1+$ & $15.8 \mathrm{~s}$ & & ० & ○ & ० & $\circ$ & $\circ$ & ○ & ○ & ० & $\circ$ & ० & ० & o & $\circ$ & $\circ$ & $\circ$ & $\circ$ & $\circ$ & ○ & o & ० & $\circ$ & o \\
\hline${ }^{100 m 1} \mathrm{Tc}$ & $4+$ & $8.32 \mu \mathrm{s}$ & 200.67 & ○ & o & ○ & $\circ$ & ○ & $\circ$ & ○ & ○ & ○ & o & ○ & o & $\circ$ & $\circ$ & ○ & $\circ$ & ○ & ○ & ○ & o & $\circ$ & $\circ$ \\
\hline $100 \mathrm{~m}^{2} \mathrm{Tc}$ & $6+$ & $3.2 \mu \mathrm{s}$ & 243.96 & $\circ$ & ० & $\circ$ & ○ & $\circ$ & ○ & ○ & ० & ○ & ० & ○ & ○ & $\circ$ & $\circ$ & ○ & ○ & ○ & ○ & ○ & ० & ○ & $\circ$ \\
\hline${ }^{101 g} \mathrm{Tc}$ & $9 / 2+$ & $14.22 \mathrm{~m}$ & & $\circ$ & $\circ$ & $\circ$ & $\circ$ & ○ & ○ & $\circ$ & ० & $\circ$ & ० & ० & ○ & ○ & ○ & ○ & ○ & o & ○ & ० & ० & ○ & ○ \\
\hline${ }^{101 m} \mathrm{Tc}$ & $1 / 2-$ & $636 \mu \mathrm{s}$ & 207.53 & $\circ$ & ○ & $\circ$ & $\circ$ & ○ & ○ & ○ & ○ & ○ & ○ & ○ & ○ & ○ & $\circ$ & ○ & ○ & ○ & ○ & ○ & ○ & ○ & ○ \\
\hline${ }^{102 g} \mathrm{Tc}$ & $1+$ & $5.28 \mathrm{~s}$ & & ○ & o & $\circ$ & $\circ$ & $\circ$ & $\circ$ & $\circ$ & ○ & $\circ$ & ○ & ○ & o & $\circ$ & $\circ$ & $\circ$ & $\circ$ & ○ & ○ & ○ & ○ & $\circ$ & ○ \\
\hline${ }^{102 m} \mathrm{Tc}$ & $5+$ & $4.35 \mathrm{~m}$ & $0.0+$ & $\circ$ & ○ & $\circ$ & ○ & ○ & $\circ$ & ○ & ० & ० & ० & ○ & ○ & ○ & ○ & ○ & ○ & ○ & $\circ$ & ० & ० & ○ & $\circ$ \\
\hline${ }^{103} \mathrm{Tc}$ & $5 / 2+$ & $54.2 \mathrm{~s}$ & & ○ & ○ & ○ & ○ & ○ & ○ & $\circ$ & ○ & ○ & ० & ○ & ○ & ○ & $\circ$ & ○ & ○ & ○ & ○ & ○ & ○ & ○ & $\circ$ \\
\hline${ }^{104 g} \mathrm{Tc}$ & $3+$ & $18.3 \mathrm{~m}$ & & ○ & $\circ$ & ○ & $\circ$ & $\circ$ & ○ & $\circ$ & $\circ$ & ○ & ○ & o & o & $\circ$ & $\circ$ & $\circ$ & ○ & ○ & $\circ$ & ○ & ○ & ○ & $\circ$ \\
\hline${ }^{104 m} \mathrm{Tc}$ & $5+$ & $3.5 \mu \mathrm{s}$ & 69.70 & $\circ$ & $\circ$ & $\circ$ & $\circ$ & ○ & ○ & $\circ$ & ○ & ○ & ○ & ○ & ○ & ○ & $\circ$ & ○ & ○ & ○ & ○ & ○ & $\circ$ & $\circ$ & $\circ$ \\
\hline
\end{tabular}

\begin{tabular}{|c|c|c|c|c|c|c|c|c|c|c|c|c|c|c|c|c|c|c|c|c|c|c|c|c|c|}
\hline${ }^{95} \mathrm{Ru}$ & $5 / 2+$ & $1.643 \mathrm{~h}$ & & 0 & 0 & 0 & & 0 & 0 & 0 & 0 & 0 & 0 & 0 & 0 & o & 0 & 0 & 0 & 0 & 0 & & 0 & o & $\circ$ \\
\hline${ }^{96} \mathrm{Ru}$ & $0+$ & $5.54 \%$ & & o & o & 0 & 0 & o & ० & 0 & o & o & 0 & o & O & o & 0 & o & ० & o & 0 & 0 & 0 & 0 & ○ \\
\hline${ }^{97} \mathrm{Ru}$ & $5 / 2+$ & $2.791 \mathrm{~d}$ & & ० & ○ & 0 & 0 & $\circ$ & o & 0 & 0 & ○ & $\circ$ & ० & ० & o & 0 & ० & o & ○ & ○ & ○ & 0 & $\circ$ & ○ \\
\hline${ }^{98} \mathrm{Ru}$ & $0+$ & $1.87 \%$ & & ० & ○ & ○ & ० & ○ & ० & ० & ○ & $\circ$ & ○ & ० & ० & o & $\circ$ & ० & o & $\circ$ & ○ & ○ & 0 & ० & ○ \\
\hline${ }^{99} \mathrm{Ru}$ & $5 / 2+$ & $12.76 \%$ & & ० & ० & 0 & $\circ$ & $\circ$ & o & o & o & $\circ$ & $\circ$ & ० & ० & o & $\circ$ & ० & o & ० & o & ○ & o & ० & ○ \\
\hline${ }^{100} \mathrm{Ru}$ & $0+$ & $12.60 \%$ & & ० & ० & 0 & ○ & ○ & ० & ० & ० & o & $\circ$ & o & o & o & ○ & o & ० & o & ० & $\circ$ & ○ & ० & $\circ$ \\
\hline${ }^{101 g} \mathrm{Ru}$ & $5 / 2+$ & $17.06 \%$ & & ० & ० & ० & ○ & ○ & o & ○ & ० & ० & ○ & ० & ० & o & ○ & ० & ० & ○ & o & ○ & ○ & ○ & ○ \\
\hline${ }^{101 m} \mathrm{Ru}$ & $11 / 2$ & $17.5 \mu \mathrm{s}$ & 527.50 & ० & 0 & 0 & $\circ$ & $\circ$ & o & ० & ○ & ○ & $\circ$ & ० & o & o & ○ & ० & ○ & ○ & ० & $\circ$ & $\circ$ & ○ & $\circ$ \\
\hline${ }^{102} \mathrm{Ru}$ & $0+$ & $31.55 \%$ & & ○ & ० & ० & $\circ$ & $\circ$ & ० & ० & ○ & ० & $\circ$ & ○ & ○ & ० & ○ & ० & $\circ$ & ○ & o & $\circ$ & ○ & ० & $\circ$ \\
\hline${ }^{103 g} \mathrm{Ru}$ & $3 / 2+$ & $39.26 \mathrm{~d}$ & & ० & ० & ० & ○ & ○ & o & ० & ० & ० & $\circ$ & ० & o & o & ○ & o & ० & ० & ० & $\circ$ & $\circ$ & ० & ○ \\
\hline${ }^{103 m} \mathrm{Ru}$ & $11 / 2$ & $1.69 \mathrm{~ms}$ & 238.20 & ० & o & ○ & ० & ० & ० & ○ & ० & ० & $\circ$ & ० & ० & o & ○ & ० & ○ & ० & ○ & $\circ$ & $\circ$ & ○ & ○ \\
\hline${ }^{104} \mathrm{Ru}$ & $0+$ & $18.62 \%$ & & ○ & o & o & ○ & o & ० & ० & o & o & ○ & ○ & o & o & ○ & o & ○ & ○ & ○ & ○ & ○ & ○ & ○ \\
\hline${ }^{105} \mathrm{Ru}$ & $3 / 2+$ & $4.44 \mathrm{~h}$ & & ○ & o & o & o & o & ० & ○ & o & o & $\circ$ & ० & o & o & o & 0 & o & ० & o & ○ & ○ & ० & $\circ$ \\
\hline${ }^{96 g} \mathrm{Rh}$ & $6+$ & $9.90 \mathrm{~m}$ & & 0 & 0 & 0 & & 0 & 0 & 0 & 0 & 0 & 0 & 0 & 0 & o & ○ & ० & ○ & o & o & & $\circ$ & ० & ○ \\
\hline${ }^{96 m} \mathrm{Rh}$ & $3+$ & $1.51 \mathrm{~m}$ & 52.00 & ○ & ○ & ○ & & $\circ$ & ० & o & ० & ○ & ○ & ○ & o & ○ & ○ & o & ○ & ० & o & & ○ & ० & $\circ$ \\
\hline${ }^{97}{ }^{g} \mathrm{Rh}$ & $9 / 2+$ & $30.7 \mathrm{~m}$ & & ○ & ○ & ○ & & ○ & ० & 0 & o & o & ○ & o & o & ० & ○ & 0 & ० & 0 & 0 & ० & ० & o & o \\
\hline
\end{tabular}

Continued on next page... 
Table 6: (continued)

\begin{tabular}{|c|c|c|c|c|c|c|c|c|c|c|c|c|c|c|c|c|c|c|c|c|c|c|c|c|c|}
\hline Target & $J^{\pi}$ & $t_{1 / 2}$ & Energy & $\stackrel{2}{\approx}$ & 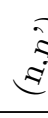 & $\begin{array}{l}\underset{\nabla}{\approx} \\
\approx\end{array}$ & 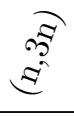 & 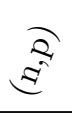 & 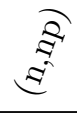 & $\underset{\nabla}{\sigma}$ & $\underset{\Xi}{\stackrel{g}{g}}$ & $\underset{\Xi}{\approx}$ & $\stackrel{A}{E}$ & 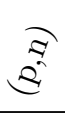 & $\begin{array}{l}\stackrel{\nabla}{\vartheta} \\
\stackrel{2}{2}\end{array}$ & $\widehat{\hat{Q}}$ & $\underset{\Omega}{\Xi}$ & $\stackrel{2}{2}$ & $\stackrel{1}{8}$ & $\underset{\Xi}{\overparen{Z}}$ & $\underset{\nabla}{\stackrel{\nabla}{\sigma}}$ & 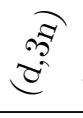 & হ & $\underset{j}{i}$ & $\underset{t}{\overparen{t}}$ \\
\hline${ }^{97 m} \mathrm{Rh}$ & $1 / 2-$ & $46.2 \mathrm{~m}$ & 258.85 & ○ & ○ & $\circ$ & & ○ & ○ & ○ & o & o & $\circ$ & ○ & o & ○ & $\circ$ & ○ & ○ & ○ & $\circ$ & ○ & $\circ$ & ○ & $\circ$ \\
\hline${ }^{98}{ }^{g} \mathrm{Rh}$ & $2+$ & $8.7 \mathrm{~m}$ & & o & o & o & 0 & ○ & ○ & o & o & ○ & o & o & o & ○ & ○ & ○ & ○ & o & o & o & o & ○ & o \\
\hline${ }^{98 m} \mathrm{Rh}$ & $5+$ & $3.5 \mathrm{~m}$ & $0.0+$ & ० & ० & $\circ$ & ○ & ○ & ○ & $\circ$ & ० & $\circ$ & $\circ$ & ० & ○ & ○ & ○ & o & ○ & ○ & $\circ$ & $\circ$ & ० & ○ & $\circ$ \\
\hline${ }^{99}{ }^{g} \mathrm{Rh}$ & $1 / 2$ & $16.1 \mathrm{~d}$ & & ○ & ○ & $\circ$ & $\circ$ & ० & ○ & ○ & o & o & $\circ$ & o & ० & ○ & ○ & ○ & ○ & ○ & $\circ$ & o & ० & ○ & $\circ$ \\
\hline${ }^{99 m} \mathrm{Rh}$ & $9 / 2+$ & $4.7 \mathrm{~h}$ & 64.30 & ○ & ○ & $\circ$ & $\circ$ & ○ & ○ & ○ & ○ & ○ & $\circ$ & ○ & ○ & ○ & ○ & ○ & ○ & ○ & ○ & ○ & ○ & ○ & $\circ$ \\
\hline${ }^{100 g} \mathrm{Rh}$ & $1-$ & $20.8 \mathrm{~h}$ & & $\circ$ & $\circ$ & $\circ$ & $\circ$ & ○ & ○ & ○ & o & ○ & $\circ$ & $\circ$ & $\circ$ & $\circ$ & ○ & ○ & ○ & ○ & ○ & ○ & $\circ$ & ○ & $\circ$ \\
\hline${ }^{100 m} \mathrm{Rh}$ & $5+$ & $4.6 \mathrm{~m}$ & 265.00 & & & & & & & & & & & & & & & & & & & & & & \\
\hline${ }^{101 g} \mathrm{Rh}$ & $1 / 2$ & $3.3 \mathrm{y}$ & & o & ○ & o & o & o & o & o & o & ○ & ○ & o & o & o & o & o & o & o & o & ○ & ○ & o & o \\
\hline${ }^{101 m} \mathrm{Rh}$ & $9 / 2+$ & $4.34 \mathrm{~d}$ & 157.32 & ○ & ○ & ○ & o & ○ & ○ & ○ & o & ○ & ○ & o & o & o & ○ & ○ & ○ & ○ & ○ & ○ & ○ & ○ & o \\
\hline${ }^{102 g} \mathrm{Rh}$ & $2-$ & $207 \mathrm{~d}$ & & ○ & o & ○ & ○ & ○ & ○ & ० & o & ○ & ○ & o & o & o & ○ & ○ & ○ & o & ० & o & o & ○ & o \\
\hline${ }^{102 m} \mathrm{Rh}$ & $6+$ & $2.9 \mathrm{y}$ & 140.75 & o & o & o & o & ○ & ○ & ○ & o & ○ & ○ & ○ & o & ○ & ○ & ○ & ○ & o & ○ & o & ○ & ○ & o \\
\hline${ }^{103 g} \mathrm{Rh}$ & $1 / 2$ & $100 \%$ & & ○ & o & ○ & ○ & ○ & ○ & o & o & o & ○ & o & o & ○ & ○ & ○ & ○ & o & o & o & o & ○ & o \\
\hline${ }^{103 m} \mathrm{Rh}$ & $7 / 2+$ & $56.114 \mathrm{~m}$ & 39.76 & $\circ$ & $\circ$ & $\circ$ & $\circ$ & $\circ$ & $\circ$ & $\circ$ & ○ & $\circ$ & $\circ$ & ० & $\circ$ & $\circ$ & $\circ$ & $\circ$ & $\circ$ & $\circ$ & $\circ$ & ० & ० & $\circ$ & $\circ$ \\
\hline${ }^{104 g} \mathrm{Rh}$ & $1+$ & $42.3 \mathrm{~s}$ & & o & o & ○ & ○ & ○ & ○ & $\circ$ & ० & ○ & $\circ$ & o & o & ○ & ○ & ○ & ○ & ○ & ○ & ○ & ० & ○ & ○ \\
\hline${ }^{104 m} \mathrm{Rh}$ & $5+$ & $4.34 \mathrm{~m}$ & 128.97 & $\circ$ & ○ & $\circ$ & $\circ$ & ० & ○ & ० & ० & $\circ$ & $\circ$ & ○ & $\circ$ & ० & ० & ० & ० & ○ & ० & ० & ० & ० & $\circ$ \\
\hline${ }^{105 g} \mathrm{Rh}$ & $7 / 2+$ & $35.36 \mathrm{~h}$ & & $\circ$ & ○ & $\circ$ & ○ & ○ & ○ & ○ & ○ & $\circ$ & $\circ$ & ○ & ○ & ○ & ○ & ○ & ○ & ○ & ० & ○ & $\circ$ & $\circ$ & ○ \\
\hline${ }^{105 m} \mathrm{Rh}$ & $1 / 2$ & $43.0 \mathrm{~s}$ & 129.78 & ○ & ○ & $\circ$ & ○ & ○ & ○ & ○ & ० & $\circ$ & $\circ$ & ○ & $\circ$ & ○ & ○ & ○ & ○ & ○ & o & ० & ○ & ○ & $\circ$ \\
\hline${ }^{106 g} \mathrm{Rh}$ & $1+$ & $29.80 \mathrm{~s}$ & & o & o & ○ & ० & ○ & ○ & ○ & ० & ○ & ○ & o & o & ○ & ○ & ○ & ○ & ○ & ○ & ० & ० & ○ & o \\
\hline${ }^{106 m} \mathrm{Rh}$ & $6+$ & $131 \mathrm{~m}$ & 137.00 & $\circ$ & $\circ$ & $\circ$ & $\circ$ & $\circ$ & $\circ$ & $\circ$ & ० & $\circ$ & $\circ$ & $\circ$ & $\circ$ & $\circ$ & $\circ$ & ○ & $\circ$ & ○ & $\circ$ & $\circ$ & $\circ$ & $\circ$ & $\circ$ \\
\hline
\end{tabular}




\section{B. Model Input Parameters}

\section{B.1. Binding and Separation Energies}

Here we present the binding energies for each of the compound nuclei included in this study. We also provide separation energies for neutrons, protons, $\alpha$-particles, and deuterons. These quantities were calculated from our adopted mass excesses, as described in section 3.1.1. All quantities are given in MeV. For isomeric states, each of these quantities should be reduced by the isomer energy.

Table 7: Binding and Separation Energies

\begin{tabular}{|c|c|c|c|c|c|}
\hline${ }^{A} \mathrm{Z}$ & Binding Energy & $S_{n}$ & $S_{p}$ & $S_{\alpha}$ & $S_{d}$ \\
\hline${ }^{75} \mathrm{Se}$ & 650.91803 & 8.02771 & 8.59808 & 4.68622 & 14.34802 \\
\hline${ }^{76} \mathrm{Se}$ & 662.07239 & 11.15436 & 9.50854 & 5.09100 & 17.52783 \\
\hline${ }^{77} \mathrm{Se}$ & 669.49121 & 7.41882 & 9.59900 & 5.72693 & 14.70276 \\
\hline${ }^{78} \mathrm{Se}$ & 679.98895 & 10.49774 & 10.39838 & 6.02856 & 17.87213 \\
\hline${ }^{79} \mathrm{Se}$ & 686.95190 & 6.96295 & 10.38928 & 6.48615 & 15.13672 \\
\hline${ }^{80} \mathrm{Se}$ & 696.86548 & 9.91357 & 11.41235 & 6.97174 & 18.07825 \\
\hline${ }^{81} \mathrm{Se}$ & 703.56635 & 6.70087 & 11.51917 & 7.60028 & 15.88861 \\
\hline${ }^{82} \mathrm{Se}$ & 712.84216 & 9.27582 & 12.34955 & 8.15668 & 18.57037 \\
\hline${ }^{83} \mathrm{Se}$ & 718.66016 & 5.81799 & 12.30554 & 8.27698 & 15.94293 \\
\hline${ }^{84} \mathrm{Se}$ & 727.34271 & 8.68256 & 13.36029 & 8.86163 & 18.76349 \\
\hline${ }^{85} \mathrm{Se}$ & 731.89038 & 4.54767 & 13.63519 & 8.54980 & 15.68335 \\
\hline${ }^{86} \mathrm{Se}$ & 738.07410 & 6.18372 & 14.50659 & 7.34155 & 17.59430 \\
\hline${ }^{87} \mathrm{Se}$ & 742.18665 & 4.11255 & 14.72083 & 8.10583 & 16.39453 \\
\hline${ }^{76} \mathrm{Br}$ & 656.32709 & 9.22137 & 5.40906 & 4.48425 & 11.21216 \\
\hline${ }^{77} \mathrm{Br}$ & 667.34418 & 11.01709 & 5.27179 & 4.70312 & 14.20154 \\
\hline${ }^{78} \mathrm{Br}$ & 675.63281 & 8.28864 & 6.14160 & 5.01721 & 11.33582 \\
\hline${ }^{79} \mathrm{Br}$ & 686.32037 & 10.68756 & 6.33142 & 5.46088 & 14.60455 \\
\hline${ }^{80} \mathrm{Br}$ & 694.21271 & 7.89233 & 7.26080 & 6.02484 & 11.99915 \\
\hline${ }^{81} \mathrm{Br}$ & 704.36938 & 10.15668 & 7.50391 & 6.48315 & 15.19287 \\
\hline${ }^{82} \mathrm{Br}$ & 711.96222 & 7.59283 & 8.39587 & 7.10394 & 12.87213 \\
\hline${ }^{83} \mathrm{Br}$ & 721.54608 & 9.58386 & 8.70392 & 7.79730 & 15.75513 \\
\hline${ }^{84} \mathrm{Br}$ & 728.40778 & 6.86169 & 9.74762 & 8.06494 & 13.34100 \\
\hline${ }^{85} \mathrm{Br}$ & 737.29010 & 8.88232 & 9.94739 & 8.50183 & 16.40533 \\
\hline${ }^{86} \mathrm{Br}$ & 742.39069 & 5.10 & 10.50031 & 7.74042 & 12.82336 \\
\hline${ }^{87} \mathrm{Br}$ & 748.67932 & 6.28 & 10.60522 & 6.40125 & 14.56433 \\
\hline${ }^{88} \mathrm{Br}$ & 753.62592 & 4.94 & 11.43927 & 7.07507 & 13.32721 \\
\hline${ }^{89} \mathrm{Br}$ & 759.53668 & 5.910 & 11.98236 & 7.67352 & 15.12543 \\
\hline${ }^{90} \mathrm{Br}$ & 763.65631 & 4.11963 & 12.71289 & 7.89484 & 13.87738 \\
\hline${ }^{77} \mathrm{Kr}$ & 663.49640 & 9.22650 & 7.16931 & 4.37677 & 14.16608 \\
\hline${ }^{78} \mathrm{Kr}$ & 675.57800 & 12.08160 & 8.23383 & 4.39203 & 17.02631 \\
\hline${ }^{79} \mathrm{Kr}$ & 683.91229 & 8.33429 & 8.27948 & 4.69861 & 14.34351 \\
\hline${ }^{80} \mathrm{Kr}$ & 695.43341 & 11.52112 & 9.11304 & 5.06537 & 17.57599 \\
\hline${ }^{81} \mathrm{Kr}$ & 703.30621 & 7.87280 & 9.09351 & 5.51935 & 14.76123 \\
\hline${ }^{82} \mathrm{Kr}$ & 714.27301 & 10.96680 & 9.90363 & 5.98840 & 17.83569 \\
\hline${ }^{83} \mathrm{Kr}$ & 721.73657 & 7.46356 & 9.77435 & 6.48901 & 15.14258 \\
\hline${ }^{84} \mathrm{Kr}$ & 732.25720 & 10.52063 & 10.71112 & 7.09607 & 18.07037 \\
\hline
\end{tabular}

Continued on next page... 
Table 7: (continued)

\begin{tabular}{|c|c|c|c|c|c|}
\hline${ }^{A} \mathrm{Z}$ & Binding Energy & $S_{n}$ & $S_{p}$ & $S_{\alpha}$ & $S_{d}$ \\
\hline${ }^{85} \mathrm{Kr}$ & 739.37769 & 7.12048 & 10.96991 & 7.51569 & 15.60699 \\
\hline${ }^{86} \mathrm{Kr}$ & 749.23438 & 9.85669 & 11.94427 & 8.09656 & 18.60199 \\
\hline${ }^{87} \mathrm{Kr}$ & 754.74951 & 5.51514 & 12.35883 & 7.79370 & 15.23480 \\
\hline${ }^{88} \mathrm{Kr}$ & 761.80359 & 7.05408 & 13.12427 & 6.16522 & 17.18829 \\
\hline${ }^{89} \mathrm{Kr}$ & 766.90942 & 5.10583 & 13.28351 & 6.72339 & 16.00549 \\
\hline${ }^{90} \mathrm{Kr}$ & 773.22388 & 6.31445 & 13.68719 & 6.85413 & 17.37335 \\
\hline${ }^{91} \mathrm{Kr}$ & 777.63568 & 4.41180 & 13.97937 & 7.15338 & 15.87439 \\
\hline${ }^{77} \mathrm{Rb}$ & 657.36908 & 12.41595 & 3.09918 & 3.62042 & 13.63660 \\
\hline${ }^{78} \mathrm{Rb}$ & 667.55212 & 10.18304 & 4.05573 & 4.05511 & 11.05762 \\
\hline${ }^{79} \mathrm{Rb}$ & 679.49060 & 11.93848 & 3.91260 & 4.08923 & 13.76959 \\
\hline${ }^{80} \mathrm{Rb}$ & 688.93140 & 9.44080 & 5.01910 & 4.30865 & 11.12878 \\
\hline${ }^{81} \mathrm{Rb}$ & 700.28473 & 11.35333 & 4.85132 & 4.64490 & 14.14783 \\
\hline${ }^{82} \mathrm{Rb}$ & 709.08942 & 8.80469 & 5.78320 & 5.16095 & 11.43140 \\
\hline${ }^{83} \mathrm{Rb}$ & 720.04730 & 10.95789 & 5.77429 & 5.43127 & 14.51648 \\
\hline${ }^{84} \mathrm{Rb}$ & 728.79382 & 8.74652 & 7.05725 & 6.28546 & 12.29620 \\
\hline${ }^{85} \mathrm{Rb}$ & 739.28247 & 10.48865 & 7.02527 & 6.61743 & 15.32129 \\
\hline${ }^{86} \mathrm{Rb}$ & 747.93347 & 8.65100 & 8.55579 & 7.67560 & 13.45166 \\
\hline${ }^{87} \mathrm{Rb}$ & 757.85559 & 9.92212 & 8.62122 & 8.01385 & 16.25330 \\
\hline${ }^{88} \mathrm{Rb}$ & 763.93811 & 6.08252 & 9.18860 & 7.23468 & 12.47913 \\
\hline${ }^{89} \mathrm{Rb}$ & 771.11292 & 7.17480 & 9.30933 & 5.52716 & 14.13879 \\
\hline${ }^{90} \mathrm{Rb}$ & 776.83337 & 5.72046 & 9.92395 & 6.14703 & 12.80518 \\
\hline${ }^{91} \mathrm{Rb}$ & 783.28839 & 6.45502 & 10.06451 & 6.31342 & 14.15436 \\
\hline${ }^{92} \mathrm{Rb}$ & 788.38641 & 5.09802 & 10.75073 & 6.46484 & 12.93793 \\
\hline${ }^{93} \mathrm{Rb}$ & 794.30322 & 5.91681 & 11.12152 & 6.47089 & 14.44293 \\
\hline${ }^{94} \mathrm{Rb}$ & 798.31030 & 4.00708 & 11.82483 & 6.35834 & 12.90399 \\
\hline${ }^{95} \mathrm{Rb}$ & 803.68219 & 5.37189 & 11.99988 & 6.77057 & 14.97211 \\
\hline${ }^{78} \mathrm{Sr}$ & 663.00751 & 13.44122 & 5.63843 & 3.26733 & 15.82977 \\
\hline${ }^{79} \mathrm{Sr}$ & 673.38147 & 10.37396 & 5.82935 & 3.57794 & 13.78778 \\
\hline${ }^{80} \mathrm{Sr}$ & 686.28442 & 12.90295 & 6.79382 & 3.71887 & 16.50769 \\
\hline${ }^{81} \mathrm{Sr}$ & 695.57520 & 9.29077 & 6.64380 & 3.78314 & 13.85999 \\
\hline${ }^{82} \mathrm{Sr}$ & 708.12720 & 12.55200 & 7.84247 & 4.25354 & 16.97119 \\
\hline${ }^{83} \mathrm{Sr}$ & 716.98560 & 8.85840 & 7.89618 & 4.77765 & 14.47626 \\
\hline${ }^{84} \mathrm{Sr}$ & 728.90527 & 11.91968 & 8.85797 & 5.17621 & 17.59125 \\
\hline${ }^{85} \mathrm{Sr}$ & 737.43542 & 8.53015 & 8.64160 & 5.83356 & 15.16351 \\
\hline${ }^{86} \mathrm{Sr}$ & 748.92767 & 11.49225 & 9.64520 & 6.35901 & 17.90924 \\
\hline${ }^{87} \mathrm{Sr}$ & 757.35577 & 8.42810 & 9.42230 & 7.32355 & 15.84869 \\
\hline${ }^{88} \mathrm{Sr}$ & 768.46851 & 11.11273 & 10.61292 & 7.91565 & 18.31042 \\
\hline${ }^{89} \mathrm{Sr}$ & 774.82721 & 6.35870 & 10.88910 & 7.15387 & 14.74701 \\
\hline${ }^{90} \mathrm{Sr}$ & 782.63098 & 7.80377 & 11.51807 & 5.10095 & 16.46826 \\
\hline${ }^{91} \mathrm{Sr}$ & 788.40601 & 5.77502 & 11.57263 & 5.36084 & 15.06848 \\
\hline${ }^{92} \mathrm{Sr}$ & 795.69971 & 7.29370 & 12.41132 & 5.60046 & 16.64172 \\
\hline${ }^{93} \mathrm{Sr}$ & 800.98792 & 5.28821 & 12.60150 & 5.78284 & 15.47491 \\
\hline${ }^{94} \mathrm{Sr}$ & 807.81512 & 6.82721 & 13.51190 & 6.29559 & 17.20410 \\
\hline
\end{tabular}

Continued on next page... 
Table 7: (continued)

\begin{tabular}{|c|c|c|c|c|c|}
\hline${ }^{A} \mathrm{Z}$ & Binding Energy & $S_{n}$ & $\overline{S_{p}}$ & $S_{\alpha}$ & $S_{d}$ \\
\hline${ }^{95} \mathrm{Sr}$ & 812.16272 & 4.34760 & 13.85242 & 6.23138 & 15.63489 \\
\hline${ }^{96} \mathrm{Sr}$ & 818.05621 & 5.89349 & 14.37402 & 6.57886 & 17.52130 \\
\hline${ }^{97} \mathrm{Sr}$ & 821.97668 & 3.92047 & 14.85236 & 7.19556 & 16.06989 \\
\hline${ }^{98} \mathrm{Sr}$ & 827.90558 & 5.92889 & 15.57837 & 7.92761 & 18.55664 \\
\hline${ }^{80} \mathrm{Y}$ & 676.41162 & 10.93250 & 3.03015 & 3.16284 & 11.17950 \\
\hline${ }^{81} \mathrm{Y}$ & 689.28247 & 12.87085 & 2.99805 & 3.61774 & 13.67639 \\
\hline${ }^{82} \mathrm{Y}$ & 699.52887 & 10.24640 & 3.95367 & 3.68109 & 11.01984 \\
\hline${ }^{83} \mathrm{Y}$ & 711.73438 & 12.20551 & 3.60718 & 3.94812 & 13.93457 \\
\hline${ }^{84} \mathrm{Y}$ & 721.63702 & 9.90265 & 4.65143 & 4.40997 & 11.28522 \\
\hline${ }^{85} \mathrm{Y}$ & 733.39258 & 11.75555 & 4.48730 & 4.81219 & 14.18237 \\
\hline${ }^{86} \mathrm{Y}$ & 742.90527 & 9.51270 & 5.46985 & 5.52020 & 11.77539 \\
\hline${ }^{87} \mathrm{Y}$ & 754.71179 & 11.80652 & 5.78412 & 6.36884 & 15.05176 \\
\hline${ }^{88} \mathrm{Y}$ & 764.06348 & 9.35168 & 6.70770 & 6.97400 & 12.91119 \\
\hline${ }^{89} \mathrm{Y}$ & 775.53748 & 11.47400 & 7.06897 & 7.95935 & 15.95709 \\
\hline${ }^{90} \mathrm{Y}$ & 782.39447 & 6.85699 & 7.56726 & 6.16534 & 11.70135 \\
\hline${ }^{91} \mathrm{Y}$ & 790.32343 & 7.92896 & 7.69244 & 4.17218 & 13.27161 \\
\hline${ }^{92} \mathrm{Y}$ & 796.86298 & 6.53955 & 8.45697 & 4.62921 & 12.00739 \\
\hline${ }^{93} \mathrm{Y}$ & 804.34412 & 7.48114 & 8.64441 & 4.93555 & 13.71350 \\
\hline${ }^{94} \mathrm{Y}$ & 810.54077 & 6.19666 & 9.55286 & 5.41174 & 12.61646 \\
\hline${ }^{95} \mathrm{Y}$ & 817.47070 & 6.92993 & 9.65558 & 5.88666 & 14.25818 \\
\hline${ }^{96} \mathrm{Y}$ & 822.68158 & 5.21088 & 10.51886 & 5.99951 & 12.64185 \\
\hline${ }^{97} \mathrm{Y}$ & 828.66388 & 5.98230 & 10.60767 & 6.06500 & 14.27655 \\
\hline${ }^{98} \mathrm{Y}$ & 832.94501 & 4.28113 & 10.96832 & 6.33905 & 12.66418 \\
\hline${ }^{99} \mathrm{Y}$ & 838.74982 & 5.80481 & 10.84424 & 6.77197 & 14.54852 \\
\hline${ }^{100} \mathrm{Y}$ & 843.91449 & 5.16467 & 12.39752 & 8.49451 & 13.78430 \\
\hline${ }^{101} \mathrm{Y}$ & 849.60370 & 5.68921 & 11.98187 & 8.98083 & 15.86212 \\
\hline${ }^{81} \mathrm{Zr}$ & 680.97131 & 11.04272 & 4.55969 & 3.10938 & 13.26758 \\
\hline${ }^{82} \mathrm{Zr}$ & 694.74615 & 13.77484 & 5.46368 & 3.44299 & 16.10992 \\
\hline${ }^{83} \mathrm{Zr}$ & 705.08398 & 10.33783 & 5.55511 & 3.40686 & 13.57690 \\
\hline${ }^{84} \mathrm{Zr}$ & 718.18884 & 13.10486 & 6.45447 & 3.60876 & 16.43536 \\
\hline${ }^{85} \mathrm{Zr}$ & 727.91718 & 9.72833 & 6.28015 & 4.04633 & 13.95819 \\
\hline${ }^{86} \mathrm{Zr}$ & 740.64380 & 12.72662 & 7.25122 & 4.22095 & 16.78217 \\
\hline${ }^{87} \mathrm{Zr}$ & 750.25891 & 9.61511 & 7.35364 & 4.97766 & 14.64172 \\
\hline${ }^{88} \mathrm{Zr}$ & 762.60510 & 12.34619 & 7.89331 & 5.40417 & 17.47522 \\
\hline${ }^{89} \mathrm{Zr}$ & 771.92218 & 9.31708 & 7.85870 & 6.19110 & 14.98578 \\
\hline${ }^{90} \mathrm{Zr}$ & 783.89197 & 11.96979 & 8.35449 & 6.66864 & 17.60388 \\
\hline${ }^{91} \mathrm{Zr}$ & 791.08643 & 7.19446 & 8.69196 & 5.43500 & 13.32434 \\
\hline${ }^{92} \mathrm{Zr}$ & 799.72119 & 8.63477 & 9.39777 & 2.95703 & 15.10211 \\
\hline${ }^{93} \mathrm{Zr}$ & 806.45569 & 6.73450 & 9.59271 & 3.33282 & 13.90765 \\
\hline${ }^{94} \mathrm{Zr}$ & 814.67682 & 8.22113 & 10.33270 & 3.75018 & 15.58923 \\
\hline${ }^{95} \mathrm{Zr}$ & 821.13898 & 6.46216 & 10.59821 & 4.43732 & 14.57025 \\
\hline${ }^{96} \mathrm{Zr}$ & 828.99542 & 7.85645 & 11.52472 & 5.00006 & 16.23004 \\
\hline${ }^{97} \mathrm{Zr}$ & 834.57050 & 5.57507 & 11.88892 & 5.28693 & 14.87518 \\
\hline
\end{tabular}

Continued on next page... 
Table 7: (continued)

\begin{tabular}{|c|c|c|c|c|c|}
\hline${ }^{A} \mathrm{Z}$ & Binding Energy & $S_{n}$ & $S_{p}$ & $S_{\alpha}$ & $S_{d}$ \\
\hline${ }^{98} \mathrm{Zr}$ & 840.98212 & 6.41162 & 12.31824 & 4.87134 & 16.07593 \\
\hline${ }^{99} \mathrm{Zr}$ & 845.53497 & 4.55286 & 12.58997 & 5.07660 & 14.64648 \\
\hline${ }^{100} \mathrm{Zr}$ & 852.44220 & 6.90723 & 13.69238 & 6.09033 & 17.27258 \\
\hline${ }^{101} \mathrm{Zr}$ & 857.36639 & 4.92419 & 13.45190 & 7.09406 & 16.39197 \\
\hline${ }^{102} \mathrm{Zr}$ & 863.72290 & 6.35651 & 14.11920 & 7.52167 & 17.58380 \\
\hline${ }^{103} \mathrm{Zr}$ & 868.42383 & 4.70093 & 13.76855 & 8.61121 & 16.59552 \\
\hline${ }^{104} \mathrm{Zr}$ & 874.46411 & 6.04028 & 14.69403 & 8.54663 & 17.58423 \\
\hline${ }^{83} \mathrm{Nb}$ & 696.80170 & 14.05591 & 2.05554 & 3.02692 & 13.60577 \\
\hline${ }^{84} \mathrm{Nb}$ & 707.79340 & 10.99170 & 2.70941 & 3.08612 & 10.82263 \\
\hline${ }^{85} \mathrm{Nb}$ & 721.13489 & 13.34149 & 2.94604 & 3.55676 & 13.82629 \\
\hline${ }^{86} \mathrm{Nb}$ & 731.88342 & 10.74854 & 3.96625 & 4.05890 & 11.46997 \\
\hline${ }^{87} \mathrm{Nb}$ & 744.31152 & 12.42810 & 3.66772 & 4.28149 & 14.16974 \\
\hline${ }^{88} \mathrm{Nb}$ & 754.27283 & 9.96130 & 4.01392 & 4.34015 & 11.40442 \\
\hline${ }^{89} \mathrm{Nb}$ & 766.92139 & 12.64856 & 4.31628 & 5.23315 & 14.43787 \\
\hline${ }^{90} \mathrm{Nb}$ & 776.99860 & 10.07721 & 5.07642 & 5.79767 & 12.16888 \\
\hline${ }^{91} \mathrm{Nb}$ & 789.04608 & 12.04749 & 5.15411 & 6.03864 & 14.89929 \\
\hline${ }^{92} \mathrm{Nb}$ & 796.93329 & 7.88721 & 5.84686 & 4.57416 & 10.81671 \\
\hline${ }^{93} \mathrm{Nb}$ & 805.76459 & 8.83130 & 6.04340 & 1.93146 & 12.45355 \\
\hline${ }^{94} \mathrm{Nb}$ & 812.99213 & 7.22754 & 6.53644 & 2.30200 & 11.04633 \\
\hline${ }^{95} \mathrm{Nb}$ & 821.48077 & 8.48865 & 6.80396 & 2.86169 & 12.80048 \\
\hline${ }^{96} \mathrm{Nb}$ & 828.37390 & 6.89313 & 7.23492 & 3.21527 & 11.47247 \\
\hline${ }^{97} \mathrm{Nb}$ & 836.44720 & 8.07330 & 7.45178 & 3.80743 & 13.08362 \\
\hline${ }^{98} \mathrm{Nb}$ & 842.44135 & 5.99414 & 7.87085 & 3.60492 & 11.22131 \\
\hline${ }^{99} \mathrm{Nb}$ & 849.31110 & 6.86975 & 8.32898 & 3.54474 & 12.51599 \\
\hline${ }^{100} \mathrm{Nb}$ & 854.99481 & 5.68372 & 9.45984 & 4.01758 & 11.78809 \\
\hline${ }^{101} \mathrm{Nb}$ & 862.06897 & 7.07416 & 9.62677 & 5.10944 & 14.30939 \\
\hline${ }^{102} \mathrm{Nb}$ & 867.54553 & 5.47656 & 10.17914 & 6.30487 & 12.87872 \\
\hline${ }^{103} \mathrm{Nb}$ & 874.58649 & 7.04095 & 10.86359 & 7.54102 & 14.99548 \\
\hline${ }^{104} \mathrm{Nb}$ & 879.56439 & 4.97791 & 11.14056 & 7.35425 & 13.61688 \\
\hline${ }^{105} \mathrm{Nb}$ & 886.26471 & 6.70032 & 11.80060 & 8.36536 & 15.61627 \\
\hline${ }^{84} \mathrm{Mo}$ & 700.93811 & 16.12933 & 4.13641 & 2.71387 & 15.96771 \\
\hline${ }^{85} \mathrm{Mo}$ & 712.30640 & 11.36829 & 4.51300 & 3.03943 & 13.28009 \\
\hline${ }^{86} \mathrm{Mo}$ & 725.83112 & 13.52472 & 4.69623 & 2.78931 & 15.81311 \\
\hline${ }^{87} \mathrm{Mo}$ & 737.04102 & 11.20990 & 5.15759 & 3.66138 & 13.68152 \\
\hline${ }^{88} \mathrm{Mo}$ & 750.11737 & 13.07635 & 5.80585 & 3.63287 & 16.00934 \\
\hline${ }^{89} \mathrm{Mo}$ & 760.49261 & 10.37524 & 6.21979 & 4.27979 & 13.95648 \\
\hline${ }^{90} \mathrm{Mo}$ & 773.72729 & 13.23468 & 6.80591 & 4.78784 & 17.22986 \\
\hline${ }^{91} \mathrm{Mo}$ & 783.83551 & 10.10822 & 6.83691 & 5.28094 & 14.68951 \\
\hline${ }^{92} \mathrm{Mo}$ & 796.50763 & 12.67212 & 7.46155 & 5.60687 & 17.28442 \\
\hline${ }^{93} \mathrm{Mo}$ & 804.57739 & 8.06976 & 7.64410 & 4.35956 & 13.30670 \\
\hline${ }^{94} \mathrm{Mo}$ & 814.25500 & 9.67761 & 8.49042 & 2.06738 & 15.09711 \\
\hline${ }^{95} \mathrm{Mo}$ & 821.62408 & 7.36908 & 8.63196 & 2.24200 & 13.63489 \\
\hline${ }^{96} \mathrm{Mo}$ & 830.77838 & 9.15430 & 9.29761 & 2.76154 & 15.56165 \\
\hline
\end{tabular}

Continued on next page... 
Table 7: (continued)

\begin{tabular}{|c|c|c|c|c|c|}
\hline${ }^{A} \mathrm{Z}$ & Binding Energy & $S_{n}$ & $S_{p}$ & $S_{\alpha}$ & $S_{d}$ \\
\hline${ }^{97} \mathrm{Mo}$ & 837.59961 & 6.82123 & 9.22571 & 2.84827 & 13.89423 \\
\hline${ }^{98} \mathrm{Mo}$ & 846.24219 & 8.64258 & 9.79498 & 3.26971 & 15.64368 \\
\hline${ }^{99} \mathrm{Mo}$ & 852.16772 & 5.92554 & 9.72638 & 2.73309 & 13.49591 \\
\hline${ }^{100} \mathrm{Mo}$ & 860.45752 & 8.28979 & 11.14642 & 3.16644 & 15.79156 \\
\hline${ }^{101} \mathrm{Mo}$ & 865.85571 & 5.39819 & 10.86090 & 2.98956 & 14.32001 \\
\hline${ }^{102} \mathrm{Mo}$ & 873.97333 & 8.11761 & 11.90436 & 4.69556 & 16.75391 \\
\hline${ }^{103} \mathrm{Mo}$ & 879.33411 & 5.36078 & 11.78857 & 5.50348 & 15.04053 \\
\hline${ }^{104} \mathrm{Mo}$ & 886.88708 & 7.55298 & 12.30060 & 6.14923 & 17.11694 \\
\hline${ }^{105} \mathrm{Mo}$ & 891.96741 & 5.08032 & 12.40302 & 6.30536 & 15.15631 \\
\hline${ }^{106} \mathrm{Mo}$ & 898.95612 & 6.98871 & 12.69141 & 6.93756 & 17.16711 \\
\hline${ }^{107} \mathrm{Mo}$ & 903.71521 & 4.75909 & 13.13574 & 6.99573 & 15.22589 \\
\hline${ }^{87} \mathrm{Tc}$ & 727.68573 & 13.98633 & 1.85461 & 2.58838 & 13.15472 \\
\hline${ }^{88} \mathrm{Tc}$ & 739.34497 & 11.65924 & 2.30396 & 3.25592 & 11.28925 \\
\hline${ }^{89} \mathrm{Tc}$ & 752.55029 & 13.20532 & 2.43292 & 3.11975 & 13.28467 \\
\hline${ }^{90} \mathrm{Tc}$ & 763.98419 & 11.43390 & 3.49158 & 3.80511 & 11.64221 \\
\hline${ }^{91} \mathrm{Tc}$ & 776.83313 & 12.84894 & 3.10583 & 4.22595 & 14.11591 \\
\hline${ }^{92} \mathrm{Tc}$ & 787.85492 & 11.02179 & 4.01941 & 5.28644 & 11.90302 \\
\hline${ }^{93} \mathrm{Tc}$ & 800.59412 & 12.73920 & 4.08649 & 5.37708 & 14.53400 \\
\hline${ }^{94} \mathrm{Tc}$ & 809.21692 & 8.62280 & 4.63953 & 3.92267 & 10.48468 \\
\hline${ }^{95} \mathrm{Tc}$ & 819.15112 & 9.93420 & 4.89612 & 1.80939 & 12.34912 \\
\hline${ }^{96} \mathrm{Tc}$ & 827.02283 & 7.87170 & 5.39874 & 1.79388 & 10.54321 \\
\hline${ }^{97} \mathrm{Tc}$ & 836.49701 & 9.47418 & 5.71863 & 2.43677 & 12.64832 \\
\hline${ }^{98} \mathrm{Tc}$ & 843.77588 & 7.27887 & 6.17627 & 2.48810 & 10.77289 \\
\hline${ }^{99} \mathrm{Tc}$ & 852.74261 & 8.96674 & 6.50043 & 2.96619 & 12.91840 \\
\hline${ }^{100} \mathrm{Tc}$ & 859.50702 & 6.76440 & 7.33929 & 2.83746 & 11.04022 \\
\hline${ }^{101} \mathrm{Tc}$ & 867.89801 & 8.39099 & 7.44049 & 3.15515 & 13.50568 \\
\hline${ }^{102} \mathrm{Tc}$ & 874.19910 & 6.30109 & 8.34338 & 3.46210 & 11.51697 \\
\hline${ }^{103} \mathrm{Tc}$ & 882.30182 & 8.10272 & 8.32849 & 4.69507 & 14.22150 \\
\hline${ }^{104} \mathrm{Tc}$ & 888.26221 & 5.96039 & 8.92810 & 4.97174 & 12.06427 \\
\hline${ }^{105} \mathrm{Tc}$ & 896.13501 & 7.87280 & 9.24792 & 5.77039 & 14.57629 \\
\hline${ }^{106} \mathrm{Tc}$ & 901.69379 & 5.55878 & 9.72638 & 5.85260 & 12.58209 \\
\hline${ }^{107} \mathrm{Tc}$ & 909.09290 & 7.39911 & 10.13678 & 6.21075 & 14.90088 \\
\hline${ }^{108} \mathrm{Tc}$ & 914.01422 & 4.92133 & 10.29901 & 6.15417 & 12.83350 \\
\hline${ }^{88} \mathrm{Ru}$ & 731.49969 & 16.37939 & 3.81396 & 2.26593 & 15.57568 \\
\hline${ }^{89} \mathrm{Ru}$ & 743.43701 & 11.93732 & 4.09204 & 2.83496 & 13.52667 \\
\hline${ }^{90} \mathrm{Ru}$ & 757.30231 & 13.86530 & 4.75201 & 3.17554 & 15.73273 \\
\hline${ }^{91} \mathrm{Ru}$ & 768.72461 & 11.42230 & 4.74042 & 3.38794 & 13.94971 \\
\hline${ }^{92} \mathrm{Ru}$ & 782.54590 & 13.82129 & 5.71277 & 4.13287 & 16.33710 \\
\hline${ }^{93} \mathrm{Ru}$ & 793.47479 & 10.92889 & 5.61987 & 4.68652 & 14.41705 \\
\hline${ }^{94} \mathrm{Ru}$ & 806.84851 & 13.37372 & 6.25439 & 4.82556 & 16.76898 \\
\hline${ }^{95} \mathrm{Ru}$ & 815.80170 & 8.95319 & 6.58478 & 3.67053 & 12.98297 \\
\hline${ }^{96} \mathrm{Ru}$ & 826.49530 & 10.69360 & 7.34418 & 1.69202 & 15.05377 \\
\hline${ }^{97} \mathrm{Ru}$ & 834.60681 & 8.11151 & 7.58398 & 1.73376 & 13.23108 \\
\hline
\end{tabular}

Continued on next page... 
Table 7: (continued)

\begin{tabular}{|c|c|c|c|c|c|}
\hline${ }^{A} \mathrm{Z}$ & Binding Energy & $S_{n}$ & $S_{p}$ & $S_{\alpha}$ & $S_{d}$ \\
\hline${ }^{98} \mathrm{Ru}$ & 844.79028 & 10.18347 & 8.29327 & 2.23962 & 15.54285 \\
\hline${ }^{99} \mathrm{Ru}$ & 852.25409 & 7.46381 & 8.47821 & 2.33435 & 13.53247 \\
\hline${ }^{100} \mathrm{Ru}$ & 861.92743 & 9.67334 & 9.18481 & 2.85339 & 15.92694 \\
\hline${ }^{101} \mathrm{Ru}$ & 868.72949 & 6.80206 & 9.22247 & 2.83423 & 13.76227 \\
\hline${ }^{102} \mathrm{Ru}$ & 877.94910 & 9.21960 & 10.05109 & 3.41125 & 16.21747 \\
\hline${ }^{103} \mathrm{Ru}$ & 884.18121 & 6.23212 & 9.98212 & 3.71783 & 14.05859 \\
\hline${ }^{104} \mathrm{Ru}$ & 893.08258 & 8.90137 & 10.78076 & 4.32941 & 16.65887 \\
\hline${ }^{105} \mathrm{Ru}$ & 898.99268 & 5.91010 & 10.73047 & 4.84131 & 14.46625 \\
\hline${ }^{106} \mathrm{Ru}$ & 907.45837 & 8.46570 & 11.32336 & 5.18939 & 16.97156 \\
\hline${ }^{107} \mathrm{Ru}$ & 913.13049 & 5.67212 & 11.43671 & 5.50073 & 14.77087 \\
\hline${ }^{108} \mathrm{Ru}$ & 920.95190 & 7.82141 & 11.85901 & 5.76917 & 17.03351 \\
\hline${ }^{109} \mathrm{Ru}$ & 926.20099 & 5.24908 & 12.18677 & 5.93793 & 14.88348 \\
\hline${ }^{91} \mathrm{Rh}$ & 758.38733 & 13.95844 & 1.08502 & 2.40594 & 12.72571 \\
\hline${ }^{92} \mathrm{Rh}$ & 770.71558 & 12.32825 & 1.99097 & 3.07495 & 11.18866 \\
\hline${ }^{93} \mathrm{Rh}$ & 784.59991 & 13.88434 & 2.05402 & 3.75397 & 13.65070 \\
\hline${ }^{94} \mathrm{Rh}$ & 796.43616 & 11.83624 & 2.96136 & 4.15631 & 11.66565 \\
\hline${ }^{95} \mathrm{Rh}$ & 809.90942 & 13.47327 & 3.06091 & 4.78064 & 14.21002 \\
\hline${ }^{96} \mathrm{Rh}$ & 819.32031 & 9.41089 & 3.51862 & 3.16974 & 10.24719 \\
\hline${ }^{97} \mathrm{Rh}$ & 830.30139 & 10.98108 & 3.80609 & 1.41162 & 12.27509 \\
\hline${ }^{98} \mathrm{Rh}$ & 838.95831 & 8.65692 & 4.35150 & 1.44574 & 10.23840 \\
\hline${ }^{99} \mathrm{Rh}$ & 849.42920 & 10.47089 & 4.63892 & 1.98242 & 12.59778 \\
\hline${ }^{100} \mathrm{Rh}$ & 857.51031 & 8.08112 & 5.25623 & 2.19183 & 10.49542 \\
\hline${ }^{101} \mathrm{Rh}$ & 867.40552 & 9.89520 & 5.47809 & 2.61285 & 12.92682 \\
\hline${ }^{102} \mathrm{Rh}$ & 874.84381 & 7.43829 & 6.11432 & 2.77228 & 10.69177 \\
\hline${ }^{103} \mathrm{Rh}$ & 884.16229 & 9.31848 & 6.21320 & 3.12402 & 13.20819 \\
\hline${ }^{104} \mathrm{Rh}$ & 891.16119 & 6.99890 & 6.97998 & 3.35852 & 10.98749 \\
\hline${ }^{105} \mathrm{Rh}$ & 900.12830 & 8.96710 & 7.04572 & 3.93463 & 13.72247 \\
\hline${ }^{106} \mathrm{Rh}$ & 906.71552 & 6.58722 & 7.72284 & 4.22076 & 11.40833 \\
\hline${ }^{107} \mathrm{Rh}$ & 915.28857 & 8.57306 & 7.83020 & 4.69110 & 14.07129 \\
\hline${ }^{108} \mathrm{Rh}$ & 921.51599 & 6.22742 & 8.38550 & 4.95813 & 11.83301 \\
\hline${ }^{109} \mathrm{Rh}$ & 929.57861 & 8.06262 & 8.62671 & 5.14795 & 14.22351 \\
\hline${ }^{110} \mathrm{Rh}$ & 935.41522 & 5.83661 & 9.21423 & 5.42578 & 12.23871 \\
\hline${ }^{94} \mathrm{Pd}$ & 789.06592 & 14.72229 & 4.46600 & 3.46796 & 16.12573 \\
\hline${ }^{95} \mathrm{Pd}$ & 800.93817 & 11.87225 & 4.50201 & 3.91791 & 14.11365 \\
\hline${ }^{96} \mathrm{Pd}$ & 815.08789 & 14.14972 & 5.17847 & 4.24634 & 16.42712 \\
\hline${ }^{97} \mathrm{Pd}$ & 824.72913 & 9.64124 & 5.40881 & 2.95868 & 12.59509 \\
\hline${ }^{98} \mathrm{Pd}$ & 836.30109 & 11.57196 & 5.99969 & 1.15692 & 14.75616 \\
\hline${ }^{99} \mathrm{Pd}$ & 845.26019 & 8.95911 & 6.30188 & 1.16284 & 12.73419 \\
\hline${ }^{100} \mathrm{Pd}$ & 856.37000 & 11.10980 & 6.94080 & 1.57904 & 15.18707 \\
\hline${ }^{101} \mathrm{Pd}$ & 864.64313 & 8.27313 & 7.13281 & 1.74066 & 12.98932 \\
\hline${ }^{102} \mathrm{Pd}$ & 875.21149 & 10.56836 & 7.80597 & 2.12555 & 15.47656 \\
\hline${ }^{103} \mathrm{Pd}$ & 882.83679 & 7.62531 & 7.99298 & 2.28705 & 13.20667 \\
\hline${ }^{104} \mathrm{Pd}$ & 892.81909 & 9.98230 & 8.65680 & 2.59601 & 15.75067 \\
\hline
\end{tabular}

Continued on next page... 
Table 7: (continued)

\begin{tabular}{ccrccr}
\hline \hline${ }^{A} \mathrm{Z}$ & Binding Energy & \multicolumn{1}{c}{$S_{n}$} & \multicolumn{1}{c}{$S_{p}$} & $S_{\alpha}$ & \multicolumn{1}{c}{$S_{d}$} \\
\hline${ }^{105} \mathrm{Pd}$ & 899.91321 & 7.09412 & 8.75201 & 2.88806 & 13.52631 \\
${ }^{106} \mathrm{Pd}$ & 909.47412 & 9.56091 & 9.34583 & 3.22937 & 16.08832 \\
${ }^{107} \mathrm{Pd}$ & 916.01062 & 6.53650 & 9.29510 & 3.53375 & 13.65771 \\
${ }^{108} \mathrm{Pd}$ & 925.23859 & 9.22797 & 9.95001 & 3.86035 & 16.29846 \\
${ }^{109} \mathrm{Pd}$ & 931.39221 & 6.15363 & 9.87622 & 4.10388 & 13.87903 \\
& & & & & \\
\hline & & & & & \\
${ }^{97} \mathrm{Ag}$ & 816.96667 & 14.31946 & 1.87878 & 4.07111 & 13.80389 \\
${ }^{98} \mathrm{Ag}$ & 827.27942 & 10.31274 & 2.55029 & 2.54761 & 9.96692 \\
${ }^{99} \mathrm{Ag}$ & 839.04779 & 11.76837 & 2.74670 & 0.84271 & 12.09406 \\
${ }^{100} \mathrm{Ag}$ & 848.50983 & 9.46204 & 3.24963 & 0.89386 & 9.98413 \\
${ }^{101} \mathrm{Ag}$ & 859.65692 & 11.14709 & 3.28693 & 1.05988 & 12.17212 \\
${ }^{102} \mathrm{Ag}$ & 868.76898 & 9.11206 & 4.12585 & 1.51501 & 10.17438 \\
${ }^{103} \mathrm{Ag}$ & 879.36670 & 10.59772 & 4.15521 & 1.64185 & 12.49896 \\
${ }^{104} \mathrm{Ag}$ & 887.75812 & 8.39142 & 4.92133 & 1.95215 & 10.32202 \\
${ }^{105} \mathrm{Ag}$ & 897.78601 & 10.02789 & 4.96692 & 2.08484 & 12.72461 \\
${ }^{106} \mathrm{Ag}$ & 905.72668 & 7.94067 & 5.81348 & 2.58722 & 10.68298 \\
${ }^{107} \mathrm{Ag}$ & 915.26239 & 9.53571 & 5.78827 & 2.80444 & 13.12457 \\
${ }^{108} \mathrm{Ag}$ & 922.53381 & 7.27142 & 6.52319 & 3.07697 & 10.83508 \\
${ }^{109} \mathrm{Ag}$ & 931.72589 & 9.19208 & 6.48730 & 3.30194 & 13.49066 \\
${ }^{110} \mathrm{Ag}$ & 938.53510 & 6.80920 & 7.14288 & 3.52393 & 11.07190 \\
& & & & & \\
\hline
\end{tabular}




\section{B.2. Q-Values for Reactions Studied}

Here we present the $\mathrm{Q}$-values (in $\mathrm{MeV}$ ) for each of the reactions included in this study. The values provided in this table are for reactions proceeding from the ground state of the target to the ground state of the residual. The Q-value for reactions proceeding from/to other states can be obtained by adding the energy of the target state to the given Q-value and subtracting the energy of the residual state. The values are calculated from our adopted mass excesses, as described in section 3.1.1. Separate tables are provided for neutron-induced (Table 8) and charged-particle (Table 9) reactions. Inelastic scattering reactions such as $\left(n, n^{\prime}\right)$ have thresholds equal to the energy of the first excited state and are not listed in the tables.

Table 8: Neutron-induced reaction Q-values

\begin{tabular}{|c|c|c|c|c|c|c|c|c|}
\hline Target & $Q_{(n, \gamma)}$ & $Q_{(n, 2 n)}$ & $Q_{(n, 3 n)}$ & $Q_{(n, p)}$ & $Q_{(n, n p)}$ & $Q_{(n, \alpha)}$ & $Q_{(n, n \alpha)}$ & $Q_{(n, d)}$ \\
\hline${ }^{83} \mathrm{Sr}$ & 11.920 & -8.858 & -21.410 & 3.062 & -7.896 & 6.743 & -4.778 & -5.672 \\
\hline${ }^{84} \mathrm{Sr}$ & 8.530 & -11.920 & -20.778 & -0.111 & -8.858 & 2.697 & -5.176 & -6.633 \\
\hline${ }^{85} \mathrm{Sr}$ & 11.492 & -8.530 & -20.450 & 1.847 & -8.642 & 5.133 & -5.834 & -6.417 \\
\hline${ }^{86} \mathrm{Sr}$ & 8.428 & -11.492 & -20.022 & -0.994 & -9.645 & 1.105 & -6.359 & -7.421 \\
\hline${ }^{87} \mathrm{Sr}$ & 11.113 & -8.428 & -19.920 & 0.500 & -9.422 & 3.197 & -7.324 & -7.198 \\
\hline${ }^{88} \mathrm{Sr}$ & 6.359 & -11.113 & -19.541 & -4.530 & -10.613 & -0.795 & -7.916 & -8.388 \\
\hline${ }^{89} \mathrm{Sr}$ & 7.804 & -6.359 & -17.471 & -3.714 & -10.889 & 2.703 & -7.154 & -8.664 \\
\hline${ }^{90} \mathrm{Sr}$ & 5.775 & -7.804 & -14.162 & -5.798 & -11.518 & 0.414 & -5.101 & -9.293 \\
\hline${ }^{91} \mathrm{Sr}$ & 7.294 & -5.775 & -13.579 & -5.118 & -11.573 & 1.693 & -5.361 & -9.348 \\
\hline${ }^{92} \mathrm{Sr}$ & 5.288 & -7.294 & -13.069 & -7.313 & -12.411 & -0.495 & -5.600 & -10.187 \\
\hline${ }^{84} \mathrm{Y}$ & 11.756 & -9.903 & -22.108 & 7.268 & -4.651 & 6.943 & -4.410 & -2.427 \\
\hline${ }^{85} \mathrm{Y}$ & 9.513 & -11.756 & -21.658 & 4.043 & -4.487 & 3.992 & -4.812 & -2.263 \\
\hline${ }^{86} \mathrm{Y}$ & 11.807 & -9.513 & -21.268 & 6.022 & -5.470 & 5.438 & -5.520 & -3.245 \\
\hline${ }^{87} \mathrm{Y}$ & 9.352 & -11.807 & -21.319 & 2.644 & -5.784 & 2.378 & -6.369 & -3.560 \\
\hline${ }^{88} \mathrm{Y}$ & 11.474 & -9.352 & -21.158 & 4.405 & -6.708 & 3.515 & -6.974 & -4.483 \\
\hline${ }^{89} \mathrm{Y}$ & 6.857 & -11.474 & -20.826 & -0.710 & -7.069 & 0.692 & -7.959 & -4.844 \\
\hline${ }^{90} \mathrm{Y}$ & 7.929 & -6.857 & -18.331 & 0.237 & -7.567 & 3.757 & -6.165 & -5.343 \\
\hline${ }^{91} \mathrm{Y}$ & 6.540 & -7.929 & -14.786 & -1.917 & -7.692 & 1.910 & -4.172 & -5.468 \\
\hline${ }^{92} \mathrm{Y}$ & 7.481 & -6.540 & -14.469 & -1.163 & -8.457 & 2.546 & -4.629 & -6.232 \\
\hline${ }^{93} \mathrm{Y}$ & 6.197 & -7.481 & -14.021 & -3.356 & -8.644 & 0.785 & -4.936 & -6.420 \\
\hline${ }^{86} \mathrm{Zr}$ & 9.615 & -12.727 & -22.455 & 2.261 & -7.251 & 4.637 & -4.221 & -5.027 \\
\hline${ }^{87} \mathrm{Zr}$ & 12.346 & -9.615 & -22.342 & 4.453 & -7.354 & 6.942 & -4.978 & -5.129 \\
\hline${ }^{88} \mathrm{Zr}$ & 9.317 & -12.346 & -21.961 & 1.458 & -7.893 & 3.126 & -5.404 & -5.669 \\
\hline${ }^{89} \mathrm{Zr}$ & 11.970 & -9.317 & -21.663 & 3.615 & -7.859 & 5.301 & -6.191 & -5.634 \\
\hline${ }^{90} \mathrm{Zr}$ & 7.194 & -11.970 & -21.287 & -1.497 & -8.354 & 1.759 & -6.669 & -6.130 \\
\hline${ }^{91} \mathrm{Zr}$ & 8.635 & -7.194 & -19.164 & -0.763 & -8.692 & 5.678 & -5.435 & -6.467 \\
\hline${ }^{92} \mathrm{Zr}$ & 6.734 & -8.635 & -15.829 & -2.858 & -9.398 & 3.402 & -2.957 & -7.173 \\
\hline${ }^{93} \mathrm{Zr}$ & 8.221 & -6.734 & -15.369 & -2.112 & -9.593 & 4.471 & -3.333 & -7.368 \\
\hline${ }^{94} \mathrm{Zr}$ & 6.462 & -8.221 & -14.956 & -4.136 & -10.333 & 2.025 & -3.750 & -8.108 \\
\hline${ }^{95} \mathrm{Zr}$ & 7.856 & -6.462 & -14.683 & -3.668 & -10.598 & 2.856 & -4.437 & -8.374 \\
\hline${ }^{96} \mathrm{Zr}$ & 5.575 & -7.856 & -14.319 & -6.314 & -11.525 & 0.288 & -5.000 & -9.300 \\
\hline${ }^{87} \mathrm{Nb}$ & 9.961 & -12.428 & -23.177 & 5.947 & -3.668 & 5.621 & -4.281 & -1.443 \\
\hline
\end{tabular}

Continued on next page... 
Table 8: (continued)

\begin{tabular}{|c|c|c|c|c|c|c|c|c|}
\hline Target & $Q_{(n, \gamma)}$ & $Q_{(n, 2 n)}$ & $Q_{(n, 3 n)}$ & $Q_{(n, p)}$ & $Q_{(n, n p)}$ & $Q_{(n, \alpha)}$ & $Q_{(n, n \alpha)}$ & $Q_{(n, d)}$ \\
\hline${ }^{88} \mathrm{Nb}$ & 12.649 & -9.961 & -22.389 & 8.332 & -4.014 & 7.415 & -4.340 & -1.789 \\
\hline${ }^{89} \mathrm{Nb}$ & 10.077 & -12.649 & -22.610 & 5.001 & -4.316 & 4.280 & -5.233 & -2.092 \\
\hline${ }^{90} \mathrm{Nb}$ & 12.047 & -10.077 & -22.726 & 6.893 & -5.076 & 6.009 & -5.798 & -2.852 \\
\hline${ }^{91} \mathrm{Nb}$ & 7.887 & -12.047 & -22.125 & 2.040 & -5.154 & 3.313 & -6.039 & -2.930 \\
\hline${ }^{92} \mathrm{Nb}$ & 8.831 & -7.887 & -19.935 & 2.788 & -5.847 & 6.900 & -4.574 & -3.622 \\
\hline${ }^{93} \mathrm{Nb}$ & 7.228 & -8.831 & -16.719 & 0.691 & -6.043 & 4.926 & -1.931 & -3.819 \\
\hline${ }^{94} \mathrm{Nb}$ & 8.489 & -7.228 & -16.059 & 1.685 & -6.536 & 5.627 & -2.302 & -4.312 \\
\hline${ }^{95} \mathrm{Nb}$ & 6.893 & -8.489 & -15.716 & -0.342 & -6.804 & 3.678 & -2.862 & -4.579 \\
\hline${ }^{96} \mathrm{Nb}$ & 8.073 & -6.893 & -15.382 & 0.622 & -7.235 & 4.266 & -3.215 & -5.010 \\
\hline${ }^{97} \mathrm{Nb}$ & 5.994 & -8.073 & -14.966 & -1.877 & -7.452 & 2.389 & -3.807 & -5.227 \\
\hline${ }^{98} \mathrm{Nb}$ & 6.870 & -5.994 & -14.067 & -1.459 & -7.871 & 3.325 & -3.605 & -5.646 \\
\hline${ }^{99} \mathrm{Nb}$ & 5.684 & -6.870 & -12.864 & -3.776 & -8.329 & 1.666 & -3.545 & -6.104 \\
\hline${ }^{100} \mathrm{Nb}$ & 7.074 & -5.684 & -12.553 & -2.553 & -9.460 & 1.965 & -4.018 & -7.235 \\
\hline${ }^{90} \mathrm{Mo}$ & 10.108 & -13.235 & -23.610 & 3.271 & -6.806 & 4.827 & -4.788 & -4.581 \\
\hline${ }^{91} \mathrm{Mo}$ & 12.672 & -10.108 & -23.343 & 5.211 & -6.837 & 7.065 & -5.281 & -4.612 \\
\hline${ }^{92} \mathrm{Mo}$ & 8.070 & -12.672 & -22.780 & 0.426 & & 3.710 & & -5.237 \\
\hline${ }^{93} \mathrm{Mo}$ & 9.678 & & -20.7 & 1.187 & & 7.610 & & -5.419 \\
\hline${ }^{94} \mathrm{Mo}$ & 7.369 & & & -1.263 & & 5.127 & & -6.266 \\
\hline${ }^{95} \mathrm{Mo}$ & 9.154 & & & -0.143 & & & & -6.407 \\
\hline${ }^{96} \mathrm{Mo}$ & 6.821 & & & -2.404 & -9.298 & 3.973 & & -7.073 \\
\hline${ }^{97} \mathrm{Mo}$ & 8.643 & & & -1.152 & -9.226 & 5.373 & & -7.001 \\
\hline${ }^{98} \mathrm{Mo}$ & 5.926 & -8.643 & -15.4 & -3.801 & -9.795 & 3.192 & & -7.570 \\
\hline${ }^{99} \mathrm{Mo}$ & 8.290 & -5.926 & -14.5 & -2.857 & & 5.123 & & -7.502 \\
\hline${ }^{100} \mathrm{Mo}$ & 5.398 & -8.290 & -14.2 & -5.463 & -11.1 & 2.409 & & -8.922 \\
\hline${ }^{101} \mathrm{Mo}$ & 8.118 & -5.398 & -13.688 & -3.787 & -10.861 & 3.422 & -2.990 & -8.636 \\
\hline${ }^{91} \mathrm{Tc}$ & 11.022 & -12.849 & -24.283 & 7.002 & -3.106 & 5.735 & -4.226 & -0.881 \\
\hline${ }^{92} \mathrm{Tc}$ & 12.739 & -11.022 & -23.871 & 8.653 & -4.019 & 7.362 & -5.286 & -1.795 \\
\hline${ }^{93} \mathrm{Tc}$ & 8.623 & -12.739 & -23.761 & 3.983 & -4.086 & 4.700 & -5.377 & -1.862 \\
\hline${ }^{94} \mathrm{Tc}$ & 9.934 & -8.623 & -21.362 & 5.038 & -4.640 & 8.125 & -3.923 & -2.415 \\
\hline${ }^{95} \mathrm{Tc}$ & 7.872 & -9.934 & -18.557 & 2.473 & -4.896 & 6.078 & -1.809 & -2.672 \\
\hline${ }^{96} \mathrm{Tc}$ & 9.474 & -7.872 & -17.806 & 3.756 & -5.399 & 7.037 & -1.794 & -3.174 \\
\hline${ }^{97} \mathrm{Tc}$ & 7.279 & -9.474 & -17.346 & 1.103 & -5.719 & 4.791 & -2.437 & -3.494 \\
\hline${ }^{98} \mathrm{Tc}$ & 8.967 & -7.279 & -16.753 & 2.466 & -6.176 & 6.001 & -2.488 & -3.952 \\
\hline${ }^{99} \mathrm{Tc}$ & 6.764 & -8.967 & -16.246 & -0.575 & -6.500 & 3.927 & -2.966 & -4.276 \\
\hline${ }^{100} \mathrm{Tc}$ & 8.391 & -6.764 & -15.731 & 0.951 & -7.339 & 5.236 & -2.837 & -5.115 \\
\hline${ }^{101} \mathrm{Tc}$ & 6.301 & -8.391 & -15.155 & -2.042 & -7.440 & 2.839 & -3.155 & -5.216 \\
\hline${ }^{102} \mathrm{Tc}$ & 8.103 & -6.301 & -14.692 & -0.226 & -8.343 & 3.408 & -3.462 & -6.119 \\
\hline${ }^{103} \mathrm{Tc}$ & 5.960 & -8.103 & -14.404 & -2.968 & -8.328 & 0.989 & -4.695 & -6.104 \\
\hline${ }^{104} \mathrm{Tc}$ & 7.873 & -5.960 & -14.063 & -1.375 & -8.928 & 2.102 & -4.972 & -6.703 \\
\hline${ }^{95} \mathrm{Ru}$ & 10.694 & -8.953 & -22.327 & 3.349 & -6.585 & 9.002 & -3.671 & -4.360 \\
\hline${ }^{96} \mathrm{Ru}$ & 8.112 & -10.694 & -19.647 & 0.528 & -7.344 & 6.378 & -1.692 & -5.120 \\
\hline
\end{tabular}

Continued on next page... 
Table 8: (continued)

\begin{tabular}{lrrrrrrrr}
\hline \hline Target & $Q_{(n, \gamma)}$ & \multicolumn{1}{c}{$Q_{(n, 2 n)}$} & $Q_{(n, 3 n)}$ & $Q_{(n, p)}$ & $Q_{(n, n p)}$ & $Q_{(n, \alpha)}$ & $Q_{(n, n \alpha)}$ & $Q_{(n, d)}$ \\
\hline${ }^{97} \mathrm{Ru}$ & 10.183 & -8.112 & -18.805 & 1.890 & -7.584 & 7.944 & -1.734 & -5.359 \\
${ }^{98} \mathrm{Ru}$ & 7.464 & -10.183 & -18.295 & -1.014 & -8.293 & 5.129 & -2.240 & -6.069 \\
${ }^{99} \mathrm{Ru}$ & 9.673 & -7.464 & -17.647 & 0.489 & -8.478 & 6.820 & -2.334 & -6.254 \\
${ }^{100} \mathrm{Ru}$ & 6.802 & -9.673 & -17.137 & -2.420 & -9.185 & 3.968 & -2.853 & -6.960 \\
${ }^{101} \mathrm{Ru}$ & 9.220 & -6.802 & -16.475 & -0.831 & -9.222 & 5.808 & -2.834 & -6.998 \\
${ }^{102} \mathrm{Ru}$ & 6.232 & -9.220 & -16.022 & -3.750 & -10.051 & 2.514 & -3.411 & -7.826 \\
${ }^{103} \mathrm{Ru}$ & 8.901 & -6.232 & -15.452 & -1.879 & -9.982 & 4.572 & -3.718 & -7.758 \\
${ }^{104} \mathrm{Ru}$ & 5.910 & -8.901 & -15.133 & -4.820 & -10.781 & 1.069 & -4.329 & -8.556 \\
${ }^{105} \mathrm{Ru}$ & 8.466 & -5.910 & -14.811 & -2.858 & -10.730 & 3.276 & -4.841 & -8.506 \\
& & & & & & & & \\
\hline${ }^{96} \mathrm{Rh}$ & 10.981 & -9.411 & -22.884 & 7.175 & -3.519 & 9.569 & -3.170 & -1.294 \\
${ }^{97} \mathrm{Rh}$ & 8.657 & -10.981 & -20.392 & 4.305 & -3.806 & 7.211 & -1.412 & -1.581 \\
${ }^{98} \mathrm{Rh}$ & 10.471 & -8.657 & -19.638 & 5.832 & -4.352 & 8.488 & -1.446 & -2.127 \\
${ }^{99} \mathrm{Rh}$ & 8.081 & -10.471 & -19.128 & 2.825 & -4.639 & 5.889 & -1.982 & -2.414 \\
${ }^{100} \mathrm{Rh}$ & 9.895 & -8.081 & -18.552 & 4.417 & -5.256 & 7.282 & -2.192 & -3.032 \\
${ }^{101} \mathrm{Rh}$ & 7.438 & -9.895 & -17.976 & 1.324 & -5.478 & 4.666 & -2.613 & -3.253 \\
${ }^{102} \mathrm{Rh}$ & 9.318 & -7.438 & -17.333 & 3.105 & -6.114 & 6.194 & -2.772 & -3.890 \\
${ }^{103} \mathrm{Rh}$ & 6.999 & -9.318 & -16.757 & 0.019 & -6.213 & 3.640 & -3.124 & -3.989 \\
${ }^{104} \mathrm{Rh}$ & 8.967 & -6.999 & -16.317 & 1.921 & -6.980 & 5.032 & -3.359 & -4.755 \\
${ }^{105} \mathrm{Rh}$ & 6.587 & -8.967 & -15.966 & -1.136 & -7.046 & 2.366 & -3.935 & -4.821 \\
${ }^{106} \mathrm{Rh}$ & 8.573 & -6.587 & -15.554 & 0.743 & -7.723 & 3.882 & -4.221 & -5.498 \\
& & & & & & & & \\
\hline
\end{tabular}


Table 9: Charged-particle reaction Q-values

\begin{tabular}{|c|c|c|c|c|c|c|c|c|}
\hline Target & $Q_{(p, \gamma)}$ & $Q_{(p, n)}$ & $Q_{(p, 2 n)}$ & $Q_{(p, n p)}$ & $Q_{(d, n)}$ & $Q_{(d, 2 n)}$ & $Q_{(d, 3 n)}$ & $Q_{(d, p)}$ \\
\hline${ }^{83} \mathrm{Sr}$ & 4.651 & -5.251 & -17.457 & -8.858 & 2.427 & -7.476 & -19.681 & 9.695 \\
\hline${ }^{84} \mathrm{Sr}$ & 4.487 & -7.268 & -17.171 & -11.920 & 2.263 & -9.493 & -19.395 & 6.306 \\
\hline${ }^{85} \mathrm{Sr}$ & 5.470 & -4.043 & -15.798 & -8.530 & 3.245 & -6.267 & -18.023 & 9.268 \\
\hline${ }^{86} \mathrm{Sr}$ & 5.784 & -6.022 & -15.535 & -11.492 & 3.560 & -8.247 & -17.760 & 6.204 \\
\hline${ }^{87} \mathrm{Sr}$ & 6.708 & -2.644 & -14.451 & -8.428 & 4.483 & -4.869 & -16.675 & 8.888 \\
\hline${ }^{88} \mathrm{Sr}$ & 7.069 & -4.405 & -13.757 & -11.113 & 4.844 & -6.630 & -15.981 & 4.134 \\
\hline${ }^{89} \mathrm{Sr}$ & 7.567 & 0.710 & -10.764 & -6.359 & 5.343 & -1.514 & -12.988 & 5.579 \\
\hline${ }^{90} \mathrm{Sr}$ & 7.692 & -0.237 & -7.094 & -7.804 & 5.468 & -2.461 & -9.318 & 3.550 \\
\hline${ }^{91} \mathrm{Sr}$ & 8.457 & 1.917 & -6.012 & -5.775 & 6.232 & -0.307 & -8.236 & 5.069 \\
\hline${ }^{92} \mathrm{Sr}$ & 8.644 & 1.163 & -5.376 & -7.294 & 6.420 & -1.061 & -7.601 & 3.064 \\
\hline${ }^{84} \mathrm{Y}$ & 6.280 & -3.448 & -16.553 & -9.903 & 4.056 & -5.673 & -18.778 & 9.531 \\
\hline${ }^{85} \mathrm{Y}$ & 7.251 & -5.475 & -15.204 & -11.756 & 5.027 & -7.700 & -17.428 & 7.288 \\
\hline${ }^{86} \mathrm{Y}$ & 7.354 & -2.261 & -14.988 & -9.513 & 5.129 & -4.486 & -17.213 & 9.582 \\
\hline${ }^{87} \mathrm{Y}$ & 7.893 & -4.453 & -14.068 & -11.807 & 5.669 & -6.677 & -16.293 & 7.127 \\
\hline${ }^{88} \mathrm{Y}$ & 7.859 & -1.458 & -13.805 & -9.352 & 5.634 & -3.683 & -16.029 & 9.249 \\
\hline${ }^{89} \mathrm{Y}$ & 8.354 & -3.615 & -12.932 & -11.474 & 6.130 & -5.840 & -15.157 & 4.632 \\
\hline${ }^{90} \mathrm{Y}$ & 8.692 & 1.497 & -10.472 & -6.857 & 6.467 & -0.727 & -12.697 & 5.704 \\
\hline${ }^{91} \mathrm{Y}$ & 9.398 & 0.763 & -6.431 & -7.929 & 7.173 & -1.462 & -8.656 & 4.315 \\
\hline${ }^{92} \mathrm{Y}$ & 9.593 & 2.858 & -5.777 & -6.540 & 7.368 & 0.634 & -8.001 & 5.257 \\
\hline${ }^{93} \mathrm{Y}$ & 10.333 & 2.112 & -4.623 & -7.481 & 8.108 & -0.113 & -6.848 & 3.972 \\
\hline${ }^{86} \mathrm{Zr}$ & 3.668 & -8.760 & -19.509 & -12.727 & 1.443 & -10.985 & -21.734 & 7.391 \\
\hline${ }^{87} \mathrm{Zr}$ & 4.014 & -5.947 & -18.375 & -9.615 & 1.789 & -8.172 & -20.600 & 10.122 \\
\hline${ }^{88} \mathrm{Zr}$ & 4.316 & -8.332 & -18.294 & -12.346 & 2.092 & -10.557 & -20.518 & 7.092 \\
\hline${ }^{89} \mathrm{Zr}$ & 5.076 & -5.001 & -17.649 & -9.317 & 2.852 & -7.225 & -19.874 & 9.745 \\
\hline${ }^{90} \mathrm{Zr}$ & 5.154 & -6.893 & -16.971 & -11.970 & 2.930 & -9.118 & -19.195 & 4.970 \\
\hline${ }^{91} \mathrm{Zr}$ & 5.847 & -2.040 & -14.088 & -7.194 & 3.622 & -4.265 & -16.312 & 6.410 \\
\hline${ }^{92} \mathrm{Zr}$ & 6.043 & -2.788 & -10.675 & -8.635 & 3.819 & -5.013 & -12.900 & 4.510 \\
\hline${ }^{93} \mathrm{Zr}$ & 6.536 & -0.691 & -9.522 & -6.734 & 4.312 & -2.916 & -11.747 & 5.997 \\
\hline${ }^{94} \mathrm{Zr}$ & 6.804 & -1.685 & -8.912 & -8.221 & 4.579 & -3.909 & -11.137 & 4.238 \\
\hline${ }^{95} \mathrm{Zr}$ & 7.235 & 0.342 & -8.147 & -6.462 & 5.010 & -1.883 & -10.371 & 5.632 \\
\hline${ }^{96} \mathrm{Zr}$ & 7.452 & -0.622 & -7.515 & -7.856 & 5.227 & -2.846 & -9.739 & 3.350 \\
\hline${ }^{87} \mathrm{Nb}$ & 5.806 & -7.271 & -18.480 & -12.428 & 3.581 & -9.495 & -20.705 & 7.737 \\
\hline${ }^{88} \mathrm{Nb}$ & 6.220 & -4.155 & -17.232 & -9.961 & 3.995 & -6.380 & -19.456 & 10.424 \\
\hline${ }^{89} \mathrm{Nb}$ & 6.806 & -6.429 & -16.804 & -12.649 & 4.581 & -8.653 & -19.029 & 7.853 \\
\hline${ }^{90} \mathrm{Nb}$ & 6.837 & -3.271 & -16.506 & -10.077 & 4.612 & -5.496 & -18.731 & 9.823 \\
\hline${ }^{91} \mathrm{Nb}$ & 7.462 & -5.211 & -15.319 & -12.047 & 5.237 & -7.435 & -17.543 & 5.663 \\
\hline${ }^{92} \mathrm{Nb}$ & 7.644 & -0.426 & -13.098 & -7.887 & 5.420 & -2.650 & -15.322 & 6.607 \\
\hline${ }^{93} \mathrm{Nb}$ & 8.490 & -1.187 & -9.257 & -8.831 & 6.266 & -3.412 & -11.482 & 5.003 \\
\hline${ }^{94} \mathrm{Nb}$ & 8.632 & 1.263 & -8.415 & -7.228 & 6.407 & -0.962 & -10.639 & 6.264 \\
\hline${ }^{95} \mathrm{Nb}$ & 9.298 & 0.143 & -7.226 & -8.489 & 7.073 & -2.081 & -9.450 & 4.669 \\
\hline${ }^{96} \mathrm{Nb}$ & 9.226 & 2.404 & -6.750 & -6.893 & 7.001 & 0.180 & -8.974 & 5.849 \\
\hline
\end{tabular}

Continued on next page... 
Table 9: (continued)

\begin{tabular}{lrlllllll}
\hline \hline Target & $Q_{(p, \gamma)}$ & $Q_{(p, n)}$ & $Q_{(p, 2 n)}$ & $Q_{(p, n p)}$ & $Q_{(d, n)}$ & $Q_{(d, 2 n)}$ & $Q_{(d, 3 n)}$ & $Q_{(d, p)}$ \\
\hline${ }^{97} \mathrm{Nb}$ & 9.795 & 1.152 & -5.669 & -8.073 & 7.570 & -1.072 & -7.893 & 3.770 \\
${ }^{98} \mathrm{Nb}$ & 9.726 & 3.801 & -4.842 & -5.994 & 7.502 & 1.576 & -7.066 & 4.645 \\
${ }^{99} \mathrm{Nb}$ & 11.146 & 2.857 & -3.069 & -6.870 & 8.922 & 0.632 & -5.294 & 3.459 \\
${ }^{100} \mathrm{Nb}$ & 10.861 & 5.463 & -2.827 & -5.684 & 8.636 & 3.238 & -5.052 & 4.850 \\
& & & & & & & & \\
\hline
\end{tabular}

\begin{tabular}{lrrrrrrrr}
${ }^{90} \mathrm{Mo}$ & 3.106 & -9.743 & -21.177 & -13.235 & 0.881 & -11.968 & -23.402 & 7.884 \\
${ }^{91} \mathrm{Mo}$ & 4.019 & -7.002 & -19.851 & -10.108 & 1.795 & -9.227 & -22.076 & 10.448 \\
${ }^{92} \mathrm{Mo}$ & 4.086 & -8.653 & -19.674 & -12.672 & 1.862 & -10.877 & -21.899 & 5.845 \\
${ }^{93} \mathrm{Mo}$ & 4.640 & -3.983 & -16.722 & -8.070 & 2.415 & -6.208 & -18.947 & 7.453 \\
${ }^{94} \mathrm{Mo}$ & 4.896 & -5.038 & -13.661 & -9.678 & 2.672 & -7.263 & -15.885 & 5.144 \\
${ }^{95} \mathrm{Mo}$ & 5.399 & -2.473 & -12.407 & -7.369 & 3.174 & -4.698 & -14.632 & 6.930 \\
${ }^{96} \mathrm{Mo}$ & 5.719 & -3.756 & -11.627 & -9.154 & 3.494 & -5.980 & -13.852 & 4.597 \\
${ }^{97} \mathrm{Mo}$ & 6.176 & -1.103 & -10.577 & -6.821 & 3.952 & -3.327 & -12.801 & 6.418 \\
${ }^{98} \mathrm{Mo}$ & 6.500 & -2.466 & -9.745 & -8.643 & 4.276 & -4.691 & -11.970 & 3.701 \\
${ }^{99} \mathrm{Mo}$ & 7.339 & 0.575 & -8.392 & -5.926 & 5.115 & -1.650 & -10.616 & 6.065 \\
${ }^{100} \mathrm{Mo}$ & 7.440 & -0.951 & -7.715 & -8.290 & 5.216 & -3.175 & -9.940 & 3.174 \\
${ }^{101} \mathrm{Mo}$ & 8.343 & 2.042 & -6.349 & -5.398 & 6.119 & -0.182 & -8.573 & 5.893 \\
& & & & & & & & \\
\hline
\end{tabular}

\begin{tabular}{lrrrrrrrr}
\hline${ }^{91} \mathrm{Tc}$ & 5.713 & -8.109 & -19.531 & -12.849 & 3.488 & -10.333 & -21.755 & 8.797 \\
${ }^{92} \mathrm{Tc}$ & 5.620 & -5.309 & -19.130 & -11.022 & 3.395 & -7.534 & -21.355 & 10.515 \\
${ }^{93} \mathrm{Tc}$ & 6.254 & -7.119 & -18.048 & -12.739 & 4.030 & -9.344 & -20.273 & 6.398 \\
${ }^{94} \mathrm{Tc}$ & 6.585 & -2.368 & -15.742 & -8.623 & 4.360 & -4.593 & -17.967 & 7.710 \\
${ }^{95} \mathrm{Tc}$ & 7.344 & -3.349 & -12.303 & -9.934 & 5.120 & -5.574 & -14.527 & 5.647 \\
${ }^{96} \mathrm{Tc}$ & 7.584 & -0.528 & -11.221 & -7.872 & 5.359 & -2.752 & -13.446 & 7.250 \\
${ }^{97} \mathrm{Tc}$ & 8.293 & -1.890 & -10.002 & -9.474 & 6.069 & -4.115 & -12.226 & 5.054 \\
${ }^{98} \mathrm{Tc}$ & 8.478 & 1.014 & -9.169 & -7.279 & 6.254 & -1.210 & -11.394 & 6.742 \\
${ }^{99} \mathrm{Tc}$ & 9.185 & -0.489 & -7.952 & -8.967 & 6.960 & -2.713 & -10.177 & 4.540 \\
${ }^{100} \mathrm{Tc}$ & 9.222 & 2.420 & -7.253 & -6.764 & 6.998 & 0.196 & -9.478 & 6.166 \\
${ }^{10} \mathrm{Tc}$ & 10.051 & 0.831 & -5.971 & -8.391 & 7.826 & -1.393 & -8.195 & 4.076 \\
${ }^{102} \mathrm{Tc}$ & 9.982 & 3.750 & -5.470 & -6.301 & 7.758 & 1.525 & -7.694 & 5.878 \\
${ }^{103} \mathrm{Tc}$ & 10.781 & 1.879 & -4.353 & -8.103 & 8.556 & -0.345 & -6.577 & 3.736 \\
${ }^{104} \mathrm{Tc}$ & 10.730 & 4.820 & -4.081 & -5.960 & 8.506 & 2.596 & -6.306 & 5.648 \\
& & & & & & & & \\
\hline
\end{tabular}

\begin{tabular}{lllllllll}
\hline${ }^{95} \mathrm{Ru}$ & 3.519 & -5.892 & -19.366 & -8.953 & 1.294 & -8.117 & -21.590 & 8.469 \\
${ }^{96} \mathrm{Ru}$ & 3.806 & -7.175 & -16.586 & -10.694 & 1.581 & -9.400 & -18.810 & 5.887 \\
${ }^{97} \mathrm{Ru}$ & 4.352 & -4.305 & -15.286 & -8.112 & 2.127 & -6.530 & -17.511 & 7.959 \\
${ }^{98} \mathrm{Ru}$ & 4.639 & -5.832 & -14.489 & -10.183 & 2.414 & -8.057 & -16.713 & 5.239 \\
${ }^{99} \mathrm{Ru}$ & 5.256 & -2.825 & -13.296 & -7.464 & 3.032 & -5.049 & -15.520 & 7.449 \\
${ }^{100} \mathrm{Ru}$ & 5.478 & -4.417 & -12.498 & -9.673 & 3.253 & -6.642 & -14.723 & 4.577 \\
${ }^{101} \mathrm{Ru}$ & 6.114 & -1.324 & -11.219 & -6.802 & 3.890 & -3.549 & -13.444 & 6.995 \\
${ }^{102} \mathrm{Ru}$ & 6.213 & -3.105 & -10.544 & -9.220 & 3.989 & -5.330 & -12.768 & 4.008 \\
${ }^{103} \mathrm{Ru}$ & 6.980 & -0.019 & -9.337 & -6.232 & 4.755 & -2.244 & -11.562 & 6.677 \\
${ }^{104} \mathrm{Ru}$ & 7.046 & -1.921 & -8.920 & -8.901 & 4.821 & -4.146 & -11.145 & 3.685 \\
${ }^{105} \mathrm{Ru}$ & 7.723 & 1.136 & -7.831 & -5.910 & 5.498 & -1.089 & -10.056 & 6.241 \\
& & & & & & & & \\
\hline
\end{tabular}

Continued on next page... 
Table 9: (continued)

\begin{tabular}{lcccccccc}
\hline \hline Target & $Q_{(p, \gamma)}$ & $Q_{(p, n)}$ & $Q_{(p, 2 n)}$ & $Q_{(p, n p)}$ & $Q_{(d, n)}$ & $Q_{(d, 2 n)}$ & $Q_{(d, 3 n)}$ & $Q_{(d, p)}$ \\
\hline \multicolumn{7}{c}{} \\
& & & & & & & \\
& & & & & & & \\
${ }^{96} \mathrm{Rh}$ & 5.409 & -4.232 & -18.382 & -9.411 & 3.184 & -6.457 & -20.607 & 8.756 \\
${ }^{97} \mathrm{Rh}$ & 6.000 & -5.572 & -15.214 & -10.981 & 3.775 & -7.797 & -17.438 & 6.432 \\
${ }^{98} \mathrm{Rh}$ & 6.302 & -2.657 & -14.229 & -8.657 & 4.077 & -4.882 & -16.454 & 8.246 \\
${ }^{99} \mathrm{Rh}$ & 6.941 & -4.169 & -13.128 & -10.471 & 4.716 & -6.394 & -15.353 & 5.857 \\
${ }^{100} \mathrm{Rh}$ & 7.133 & -1.140 & -12.250 & -8.081 & 4.908 & -3.365 & -14.475 & 7.671 \\
${ }^{10} \mathrm{Rh}$ & 7.806 & -2.762 & -11.036 & -9.895 & 5.581 & -4.987 & -13.260 & 5.214 \\
${ }^{102} \mathrm{Rh}$ & 7.993 & 0.368 & -10.201 & -7.438 & 5.768 & -1.857 & -12.425 & 7.094 \\
${ }^{103} \mathrm{Rh}$ & 8.657 & -1.326 & -8.951 & -9.318 & 6.432 & -3.550 & -11.175 & 4.774 \\
${ }^{104} \mathrm{Rh}$ & 8.752 & 1.658 & -8.324 & -6.999 & 6.527 & -0.567 & -10.549 & 6.743 \\
${ }^{105} \mathrm{Rh}$ & 9.346 & -0.215 & -7.309 & -8.967 & 7.121 & -2.440 & -9.534 & 4.363 \\
${ }^{106} \mathrm{Rh}$ & 9.295 & 2.759 & -6.802 & -6.587 & 7.071 & 0.534 & -9.027 & 6.348 \\
& & & & & & & & \\
\hline
\end{tabular}




\section{B.3. Modified Discrete Level Schemes}

As stated in section 3.1.2, most of our discrete level schemes are taken directly from (Belgya et al. 2005). For a few of the isotopes we have made significant modifications, such as changes in the spin and parity assignments, adjustments to the branching ratios, and the addition or removal of levels. The following figures present our modified level schemes, up to 25 levels. The modified level data for bromine, krypton, and rubidium isotopes may be found in (Hoffman et al. 2004a).

In these plots, the level energy is listed on the right hand side, and the spin and parity of the level on the left. The $\gamma$-ray transitions are indicated by arrows. Each arrow is labeled with the transition energy, followed by the branching ratio (as a percent) in parenthesis. Isomeric states are indicated by thick black lines.

We have made minor changes to a few additional nuclei not included in this appendix. See section 3.1.2 for details. 

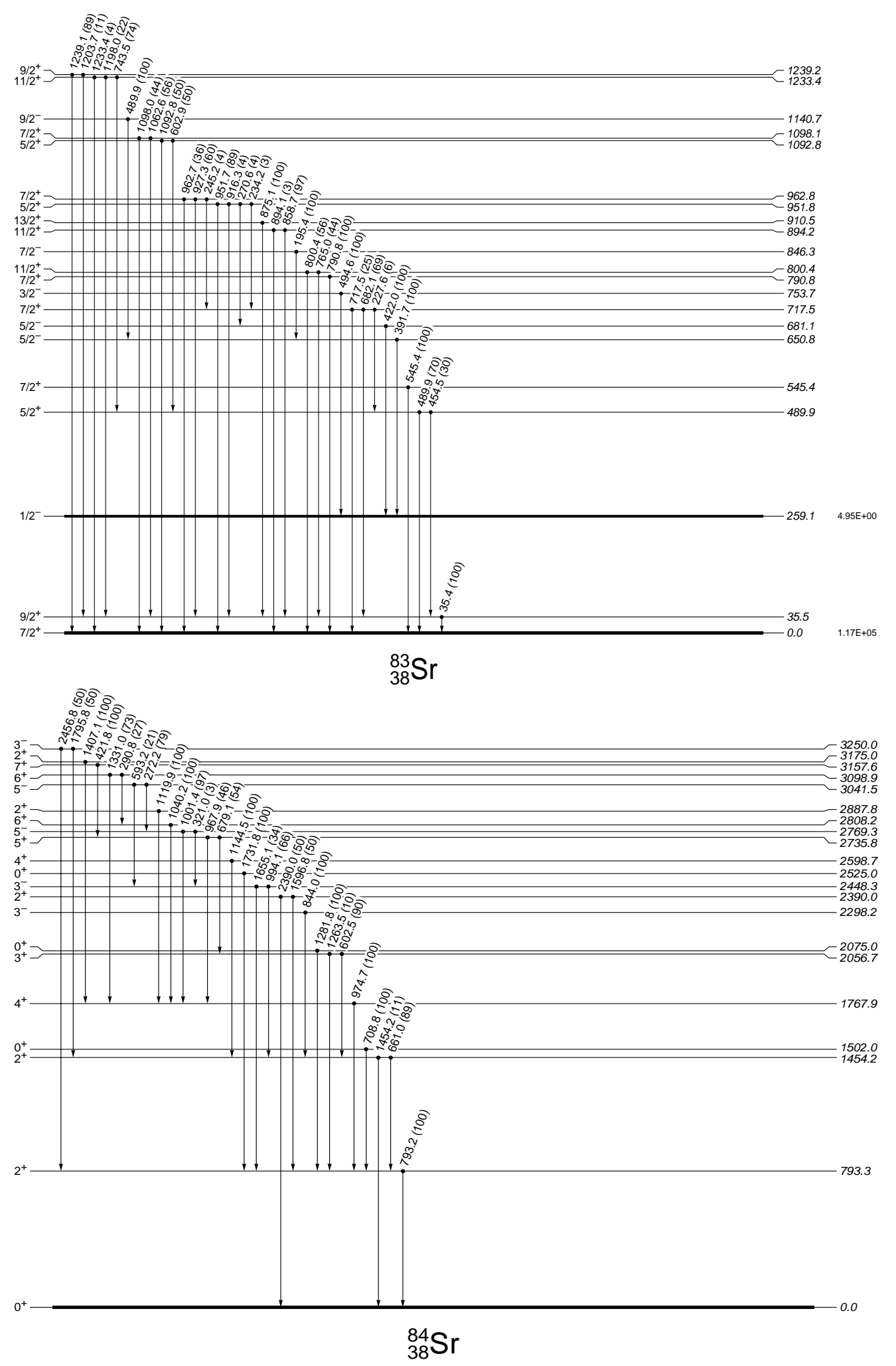

Fig. 24.- Adopted level schemes for select nuclei. 


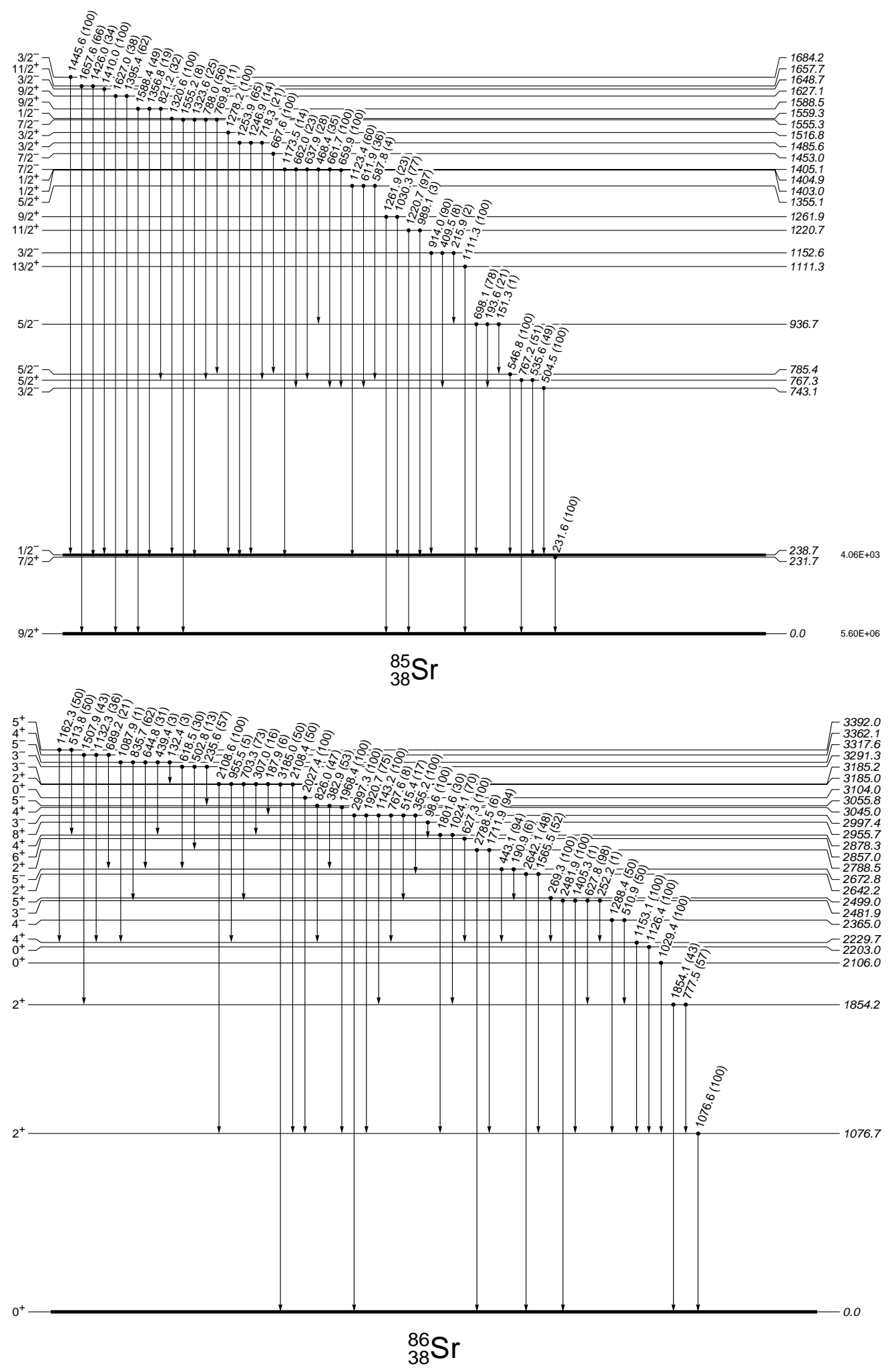

Fig. 24.- (continued) 


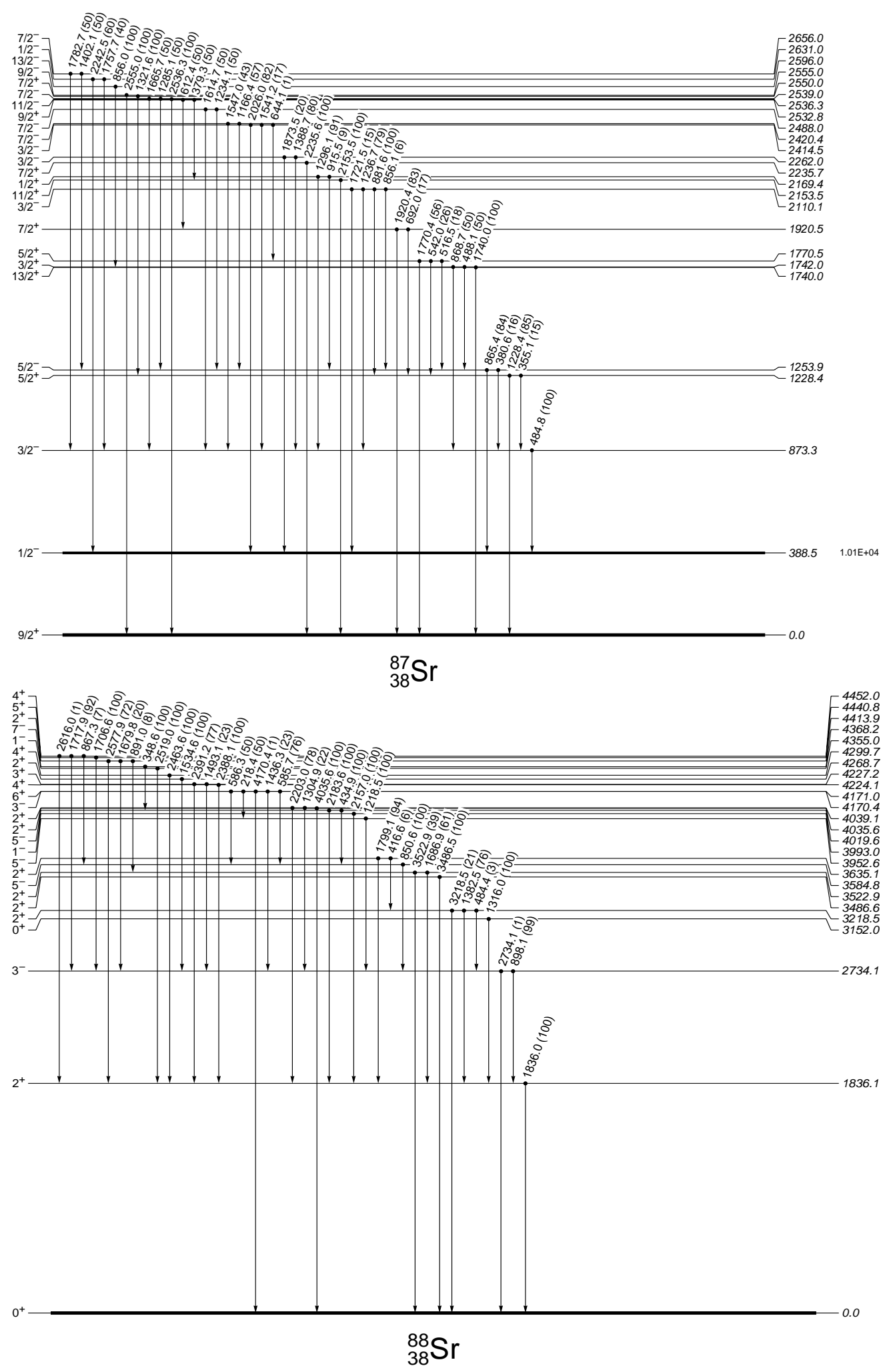

Fig. 24.- (continued) 


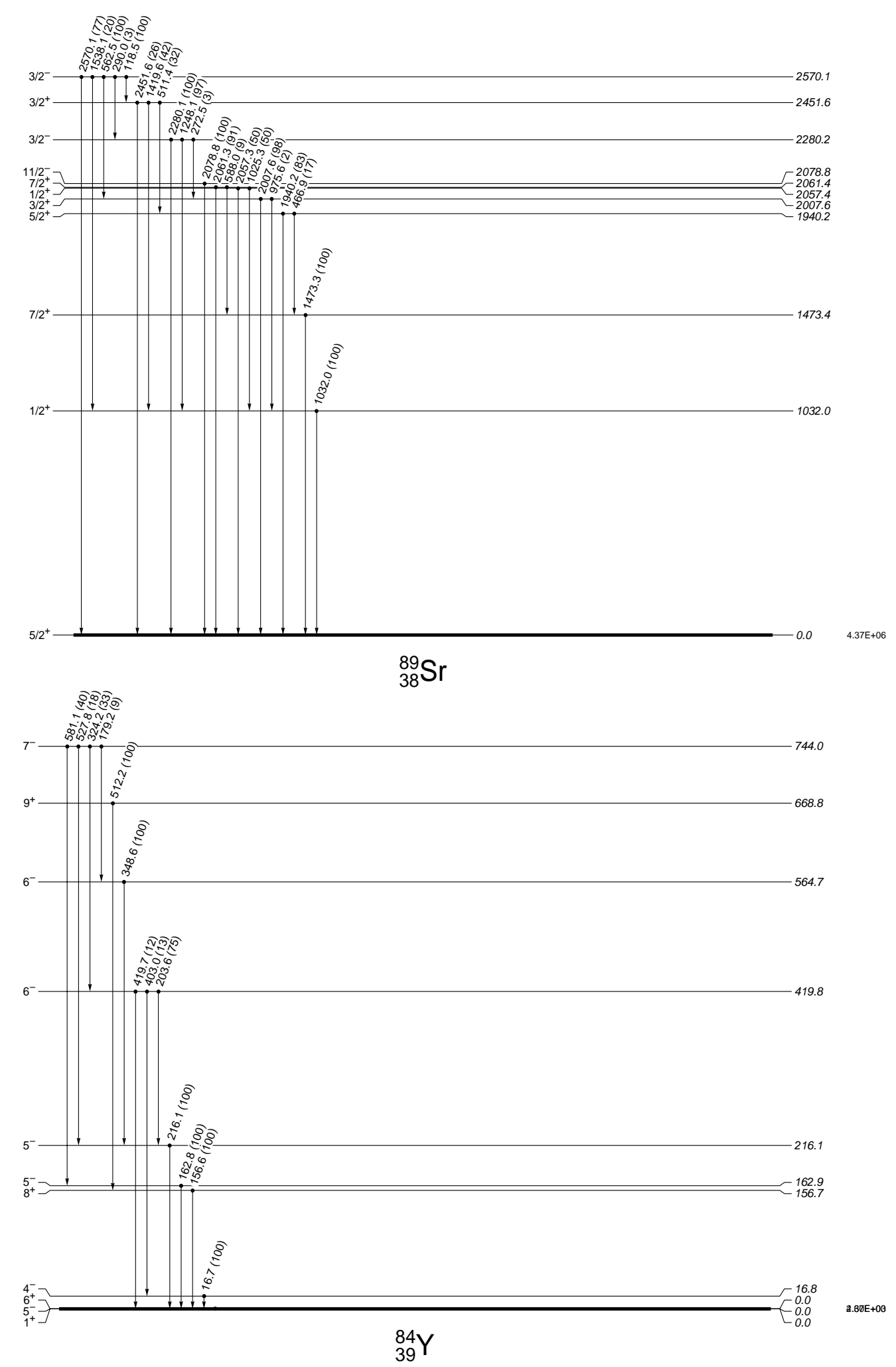

Fig. 24.- (continued) 


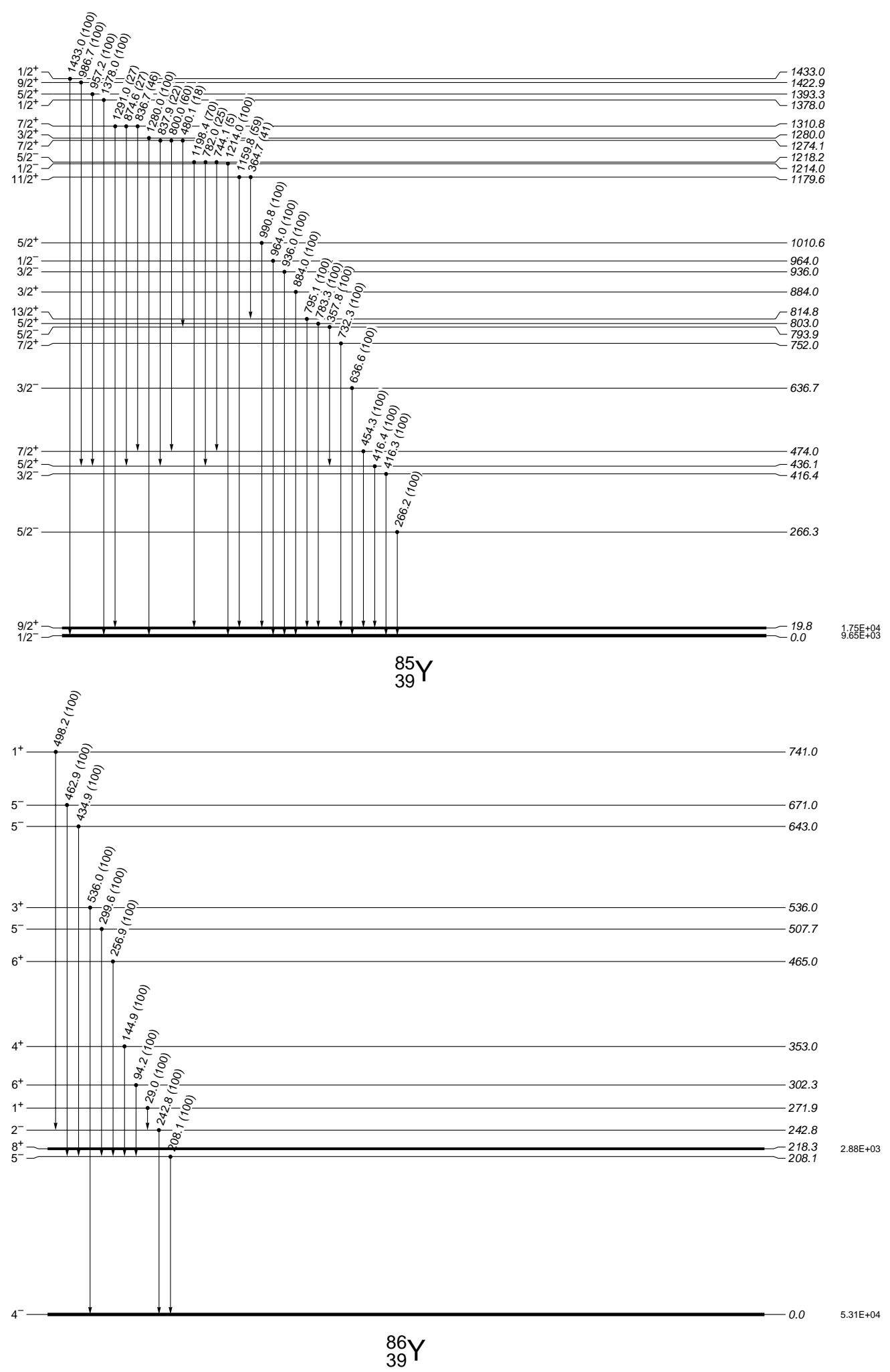

Fig. 24.- (continued) 

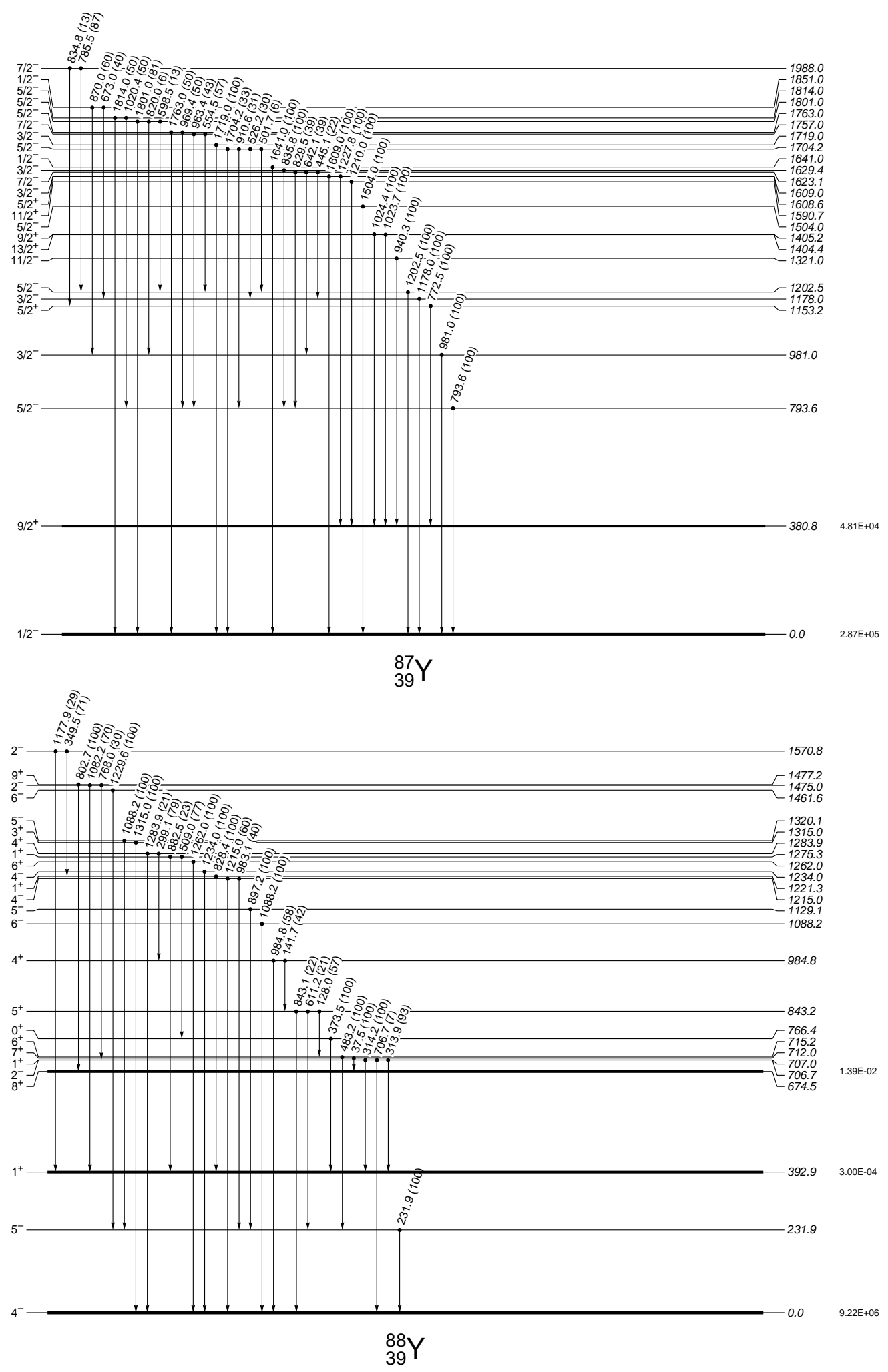

Fig. 24.- (continued) 


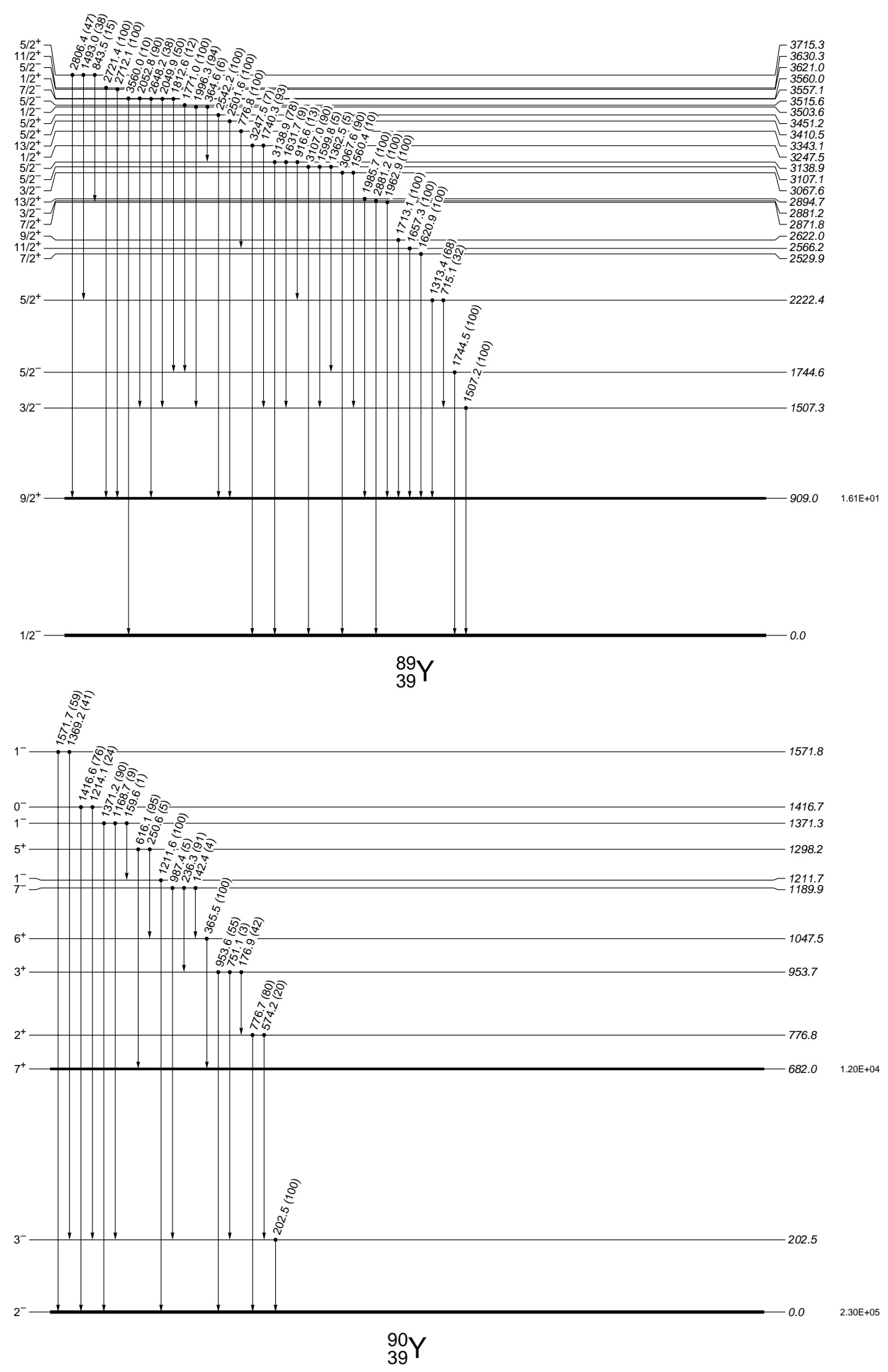

Fig. 24.- (continued) 


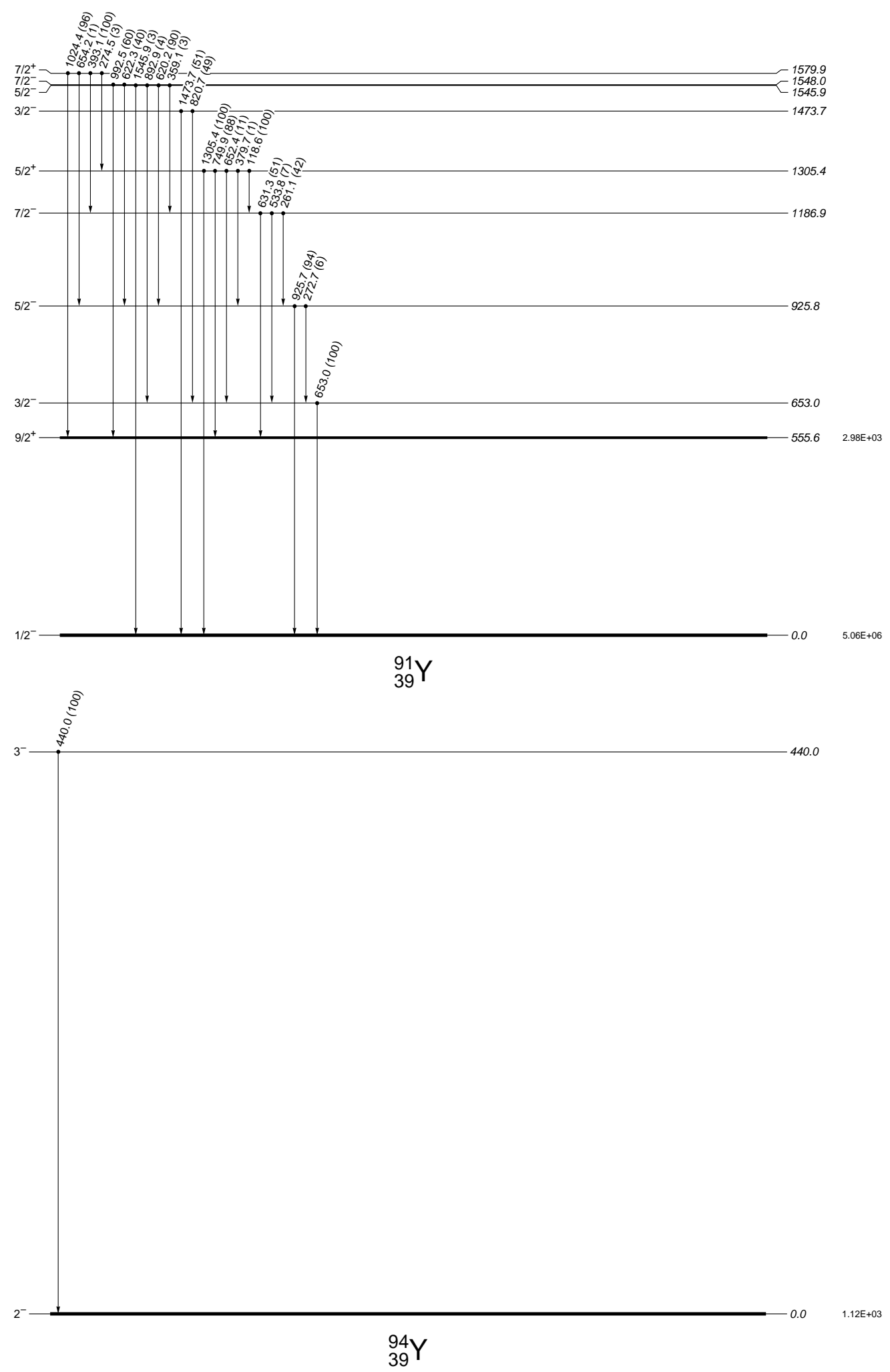

Fig. 24.- (continued) 

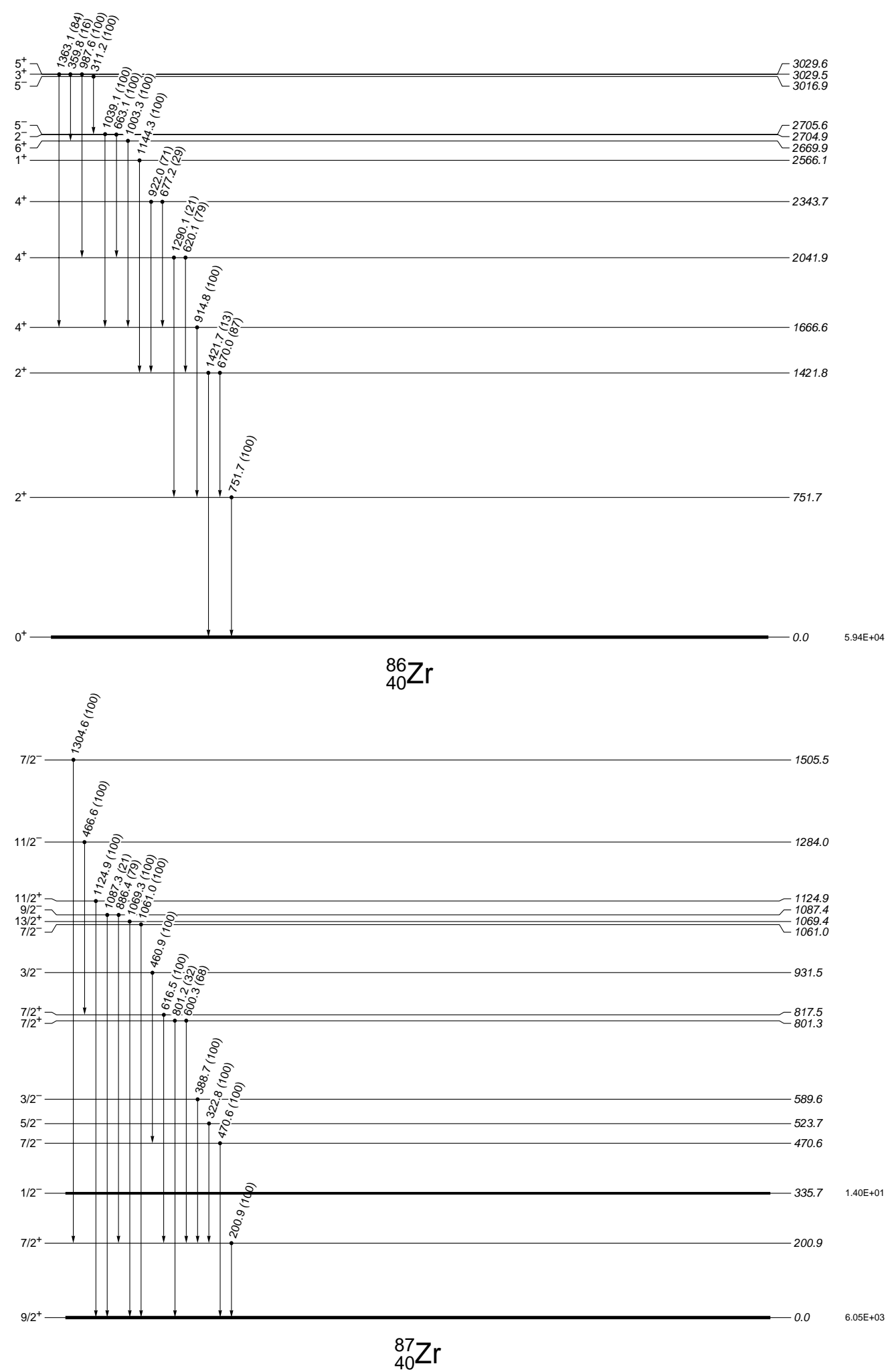

Fig. 24.- (continued) 

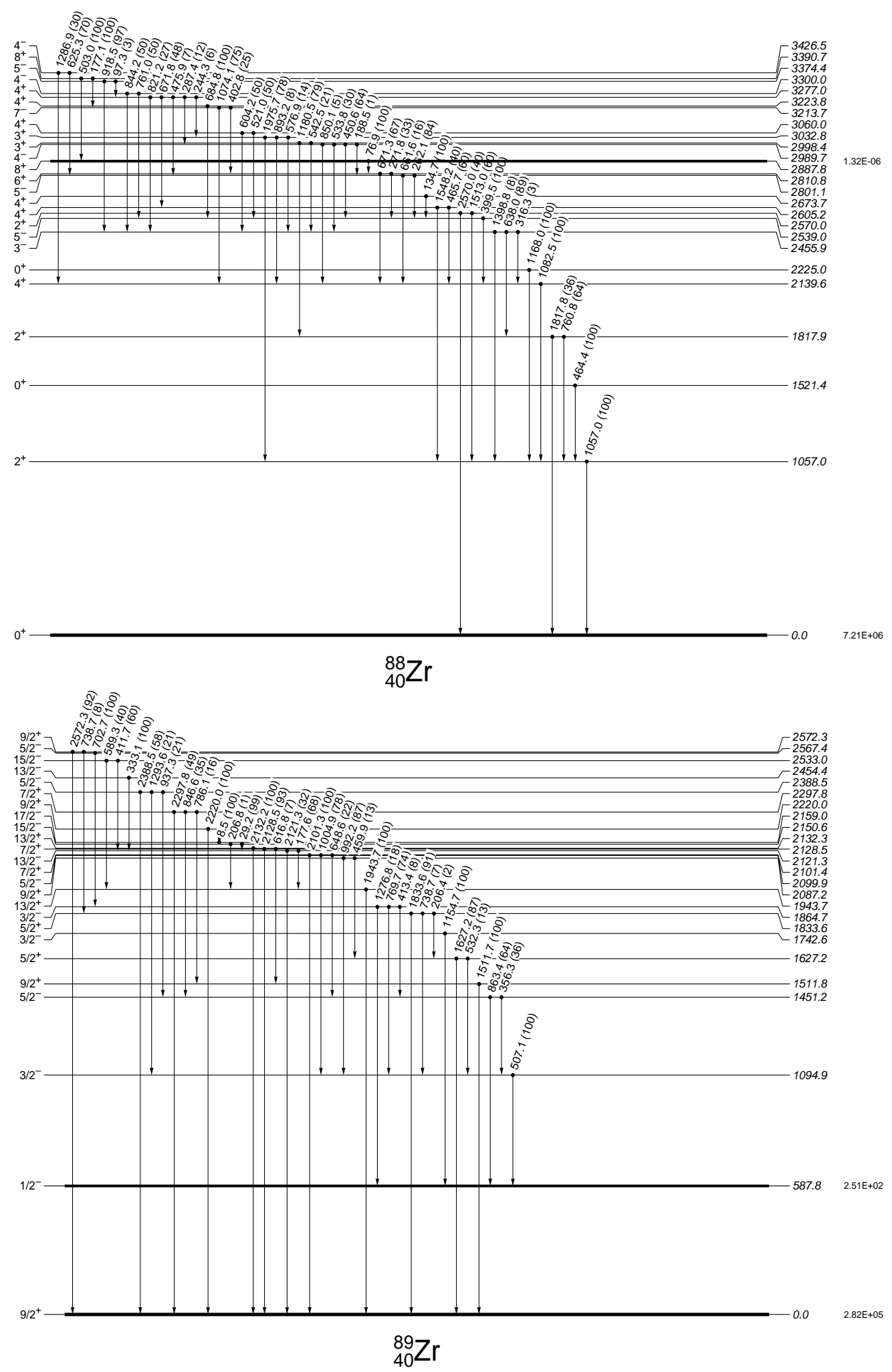

Fig. 24.- (continued) 

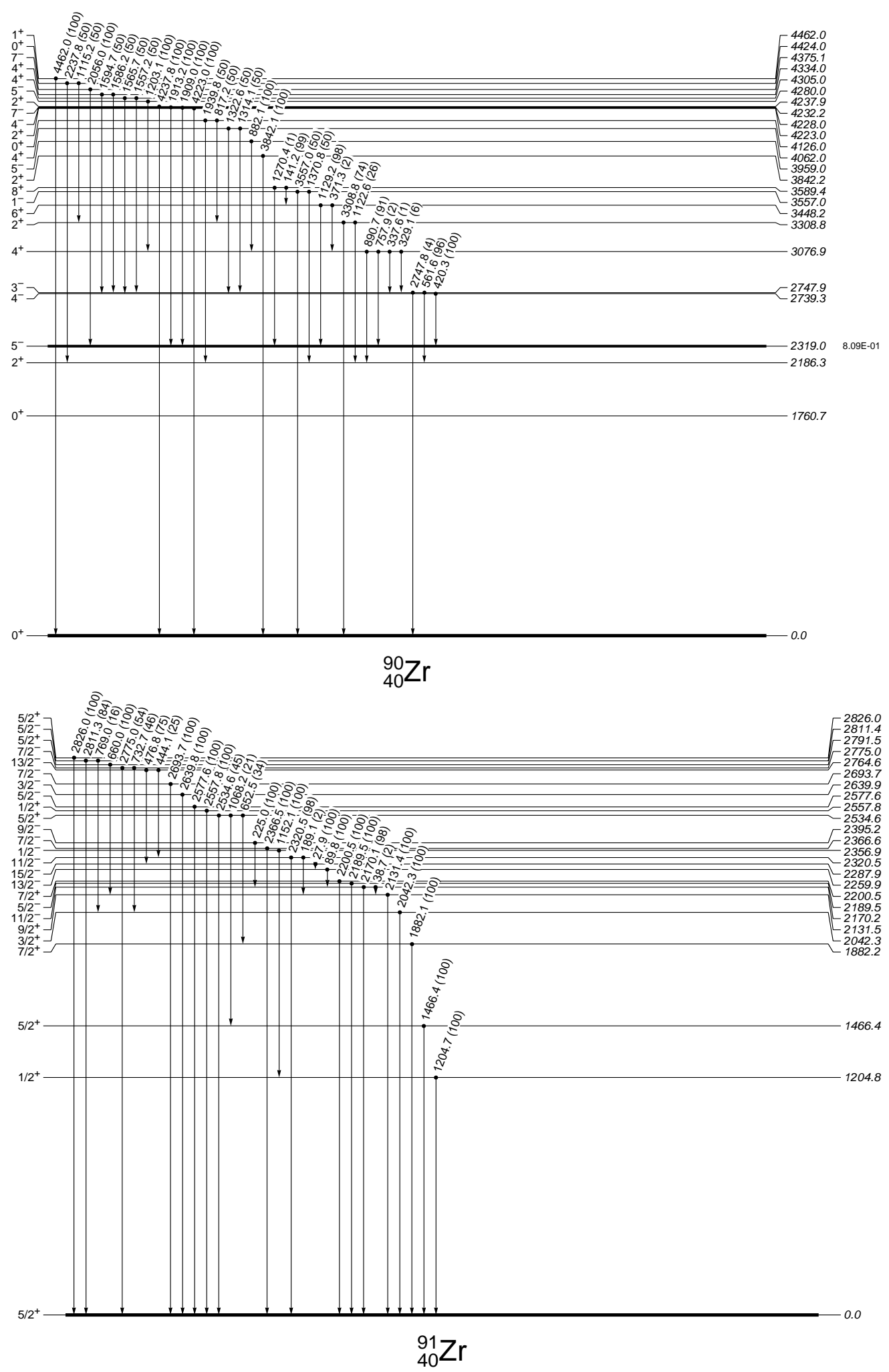

Fig. 24.- (continued) 

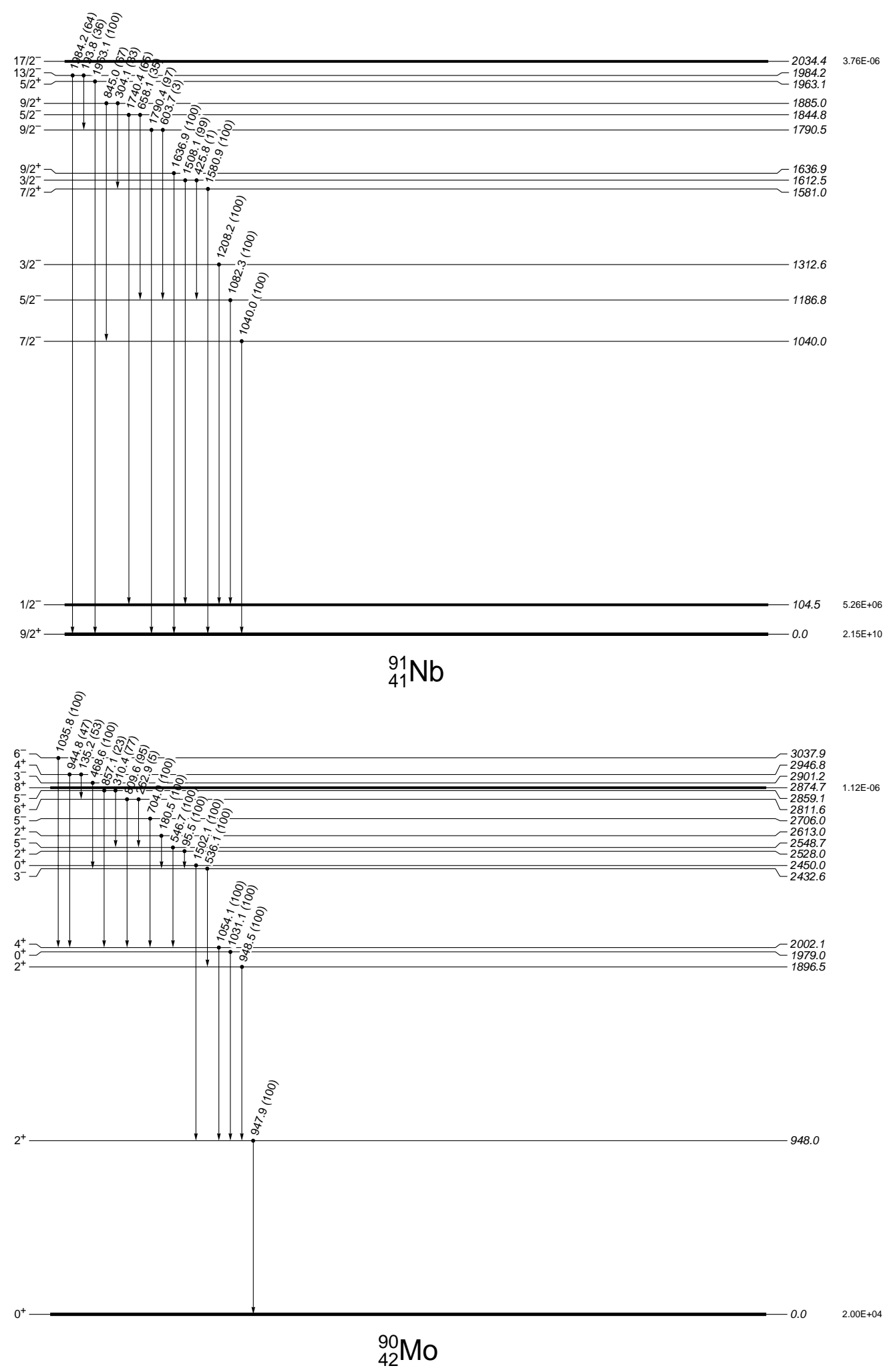

Fig. 24.- (continued) 


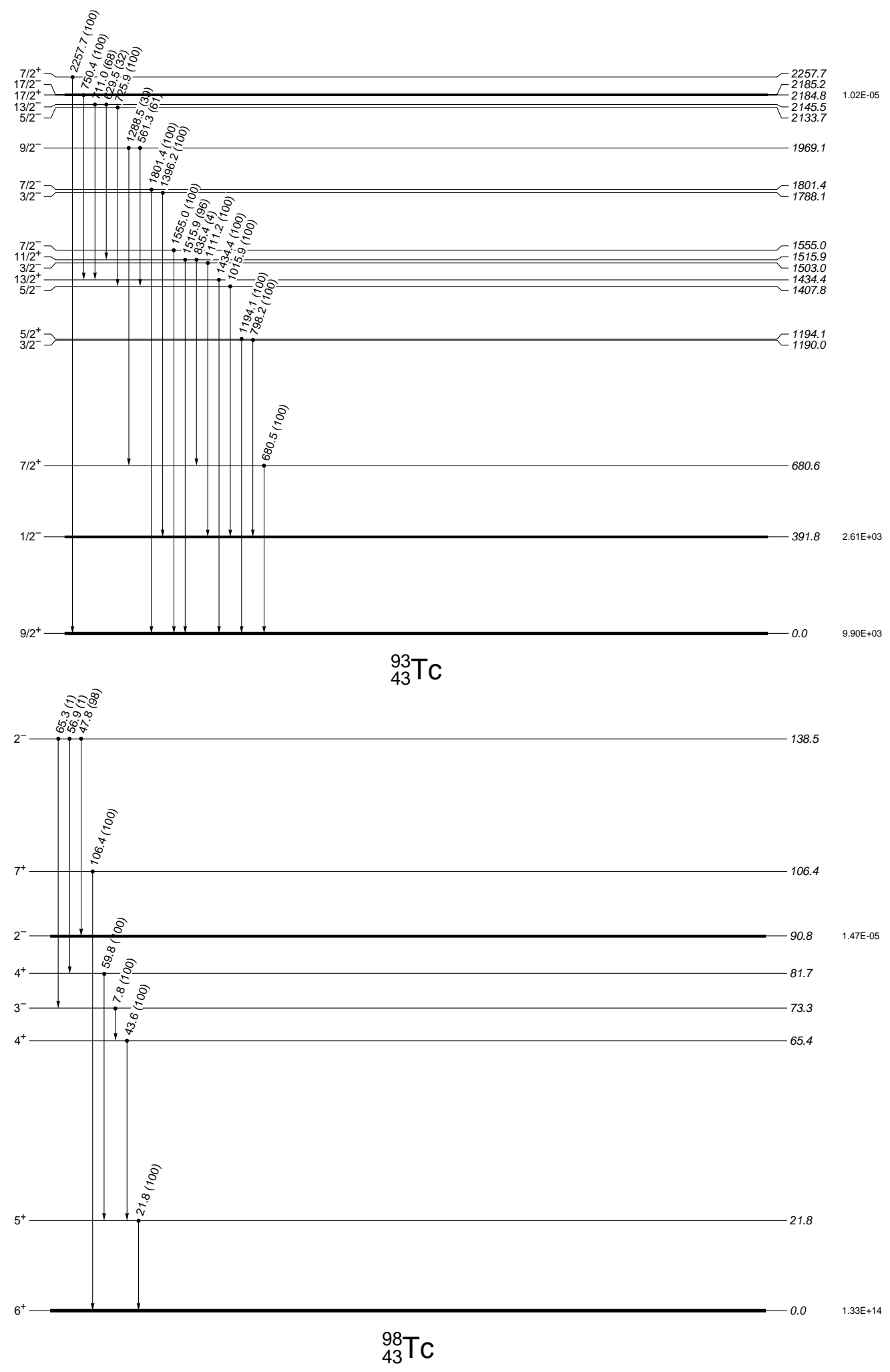

Fig. 24.- (continued) 


\section{B.4. Level Density Parameters}

Here we present the level density parameters, as described in section 3.3, for each nucleus considered as a target, compound nucleus, or possible exit channel in this study. The first column lists the nuclei included. The second column is the asymptotic level density parameter in $\mathrm{MeV}^{-1}$. Column three is the backshift in $\mathrm{MeV}$. Columns four and five are the shell correction in $\mathrm{MeV}$ and a flag that indicates whether the shell correction is based on an experimentally measured resonance spacing $(\mathrm{x})$ or is taken from systematics (s). Column six gives the matching energy. Columns seven, eight, and nine are the constant temperature parameters. The last column indicates the number of excited states to which the level density was fit, which is also the number of excited states included in our Hauser-Feshbach calculations.

Table 10: Level Density Parameters

\begin{tabular}{|c|c|c|c|c|c|c|c|c|c|}
\hline Target & $\tilde{a}$ & $\Delta$ & $\delta \bar{~} \delta W$ & $\mathrm{x} / \mathrm{s}$ & $E_{x}$ & $T$ & $\sigma\left(E_{x}\right)$ & $E_{0}$ & $N$ \\
\hline${ }^{75} \mathrm{Se}$ & 8.859 & 0.016 & 6.663 & $\mathrm{x}$ & 4.765 & 0.836 & 3.585 & -3.664 & 17 \\
\hline${ }^{76} \mathrm{Se}$ & 8.982 & 1.993 & 6.238 & $\mathrm{~s}$ & 6.887 & 0.845 & 3.654 & -1.795 & 17 \\
\hline${ }^{77} \mathrm{Se}$ & 9.106 & 0.095 & 5.947 & $\mathrm{x}$ & 4.758 & 0.826 & 3.645 & -3.488 & 17 \\
\hline${ }^{78} \mathrm{Se}$ & 9.229 & 1.900 & 5.504 & $\mathrm{x}$ & 6.560 & 0.825 & 3.686 & -1.663 & 17 \\
\hline${ }^{79} \mathrm{Se}$ & 9.352 & 0.044 & 3.062 & $\mathrm{x}$ & 6.000 & 0.944 & 4.041 & -4.459 & 1 \\
\hline${ }^{80} \mathrm{Se}$ & 9.476 & 1.780 & 3.765 & $\mathrm{~s}$ & 5.778 & 0.790 & 3.654 & -1.189 & 20 \\
\hline${ }^{81} \mathrm{Se}$ & 9.600 & 0.137 & 3.552 & $\mathrm{x}$ & 4.490 & 0.813 & 3.768 & -3.104 & 4 \\
\hline${ }^{82} \mathrm{Se}$ & 9.723 & 1.776 & 1.156 & $\mathrm{~s}$ & 3.925 & 0.676 & 3.256 & 0.180 & 8 \\
\hline${ }^{83} \mathrm{Se}$ & 9.847 & -0.077 & 2.267 & $\mathrm{x}$ & 3.000 & 0.726 & 3.545 & -2.315 & 1 \\
\hline${ }^{84} \mathrm{Se}$ & 9.970 & 1.887 & -2.368 & $\mathrm{~s}$ & 7.500 & 0.985 & 4.366 & -1.912 & 1 \\
\hline${ }^{85} \mathrm{Se}$ & 10.094 & 0.375 & -2.500 & $\mathrm{~s}$ & 4.000 & 0.850 & 3.953 & -2.097 & 0 \\
\hline${ }^{86} \mathrm{Se}$ & 10.218 & 1.493 & -1.379 & $\mathrm{~s}$ & 5.737 & 0.862 & 4.082 & -1.444 & 0 \\
\hline${ }^{87} \mathrm{Se}$ & 10.342 & 0.507 & -0.343 & $\mathrm{~s}$ & 4.731 & 0.833 & 4.056 & -2.471 & 0 \\
\hline${ }^{76} \mathrm{Br}$ & 8.982 & -0.889 & 6.284 & $\mathrm{~s}$ & 3.393 & 0.800 & 3.530 & -4.166 & 24 \\
\hline${ }^{77} \mathrm{Br}$ & 9.106 & -0.058 & 6.238 & $\mathrm{~s}$ & 4.774 & 0.833 & 3.669 & -3.796 & 25 \\
\hline${ }^{78} \mathrm{Br}$ & 9.229 & -1.134 & 5.963 & $\mathrm{~s}$ & 3.465 & 0.814 & 3.659 & -4.665 & 24 \\
\hline${ }^{79} \mathrm{Br}$ & 9.352 & 0.003 & 5.459 & $\mathrm{~s}$ & 4.586 & 0.813 & 3.698 & -3.496 & 26 \\
\hline${ }^{80} \mathrm{Br}$ & 9.476 & -1.024 & 4.753 & $\mathrm{x}$ & 1.958 & 0.695 & 3.361 & -3.238 & 7 \\
\hline${ }^{81} \mathrm{Br}$ & 9.600 & 0.041 & 3.765 & $\mathrm{~s}$ & 4.413 & 0.811 & 3.765 & -3.223 & 25 \\
\hline${ }^{82} \mathrm{Br}$ & 9.723 & -0.842 & 3.085 & $\mathrm{x}$ & 2.014 & 0.700 & 3.427 & -2.937 & 24 \\
\hline${ }^{83} \mathrm{Br}$ & 9.847 & 0.179 & 1.156 & $\mathrm{~s}$ & 1.000 & 0.579 & 2.577 & -0.763 & 25 \\
\hline${ }^{84} \mathrm{Br}$ & 9.970 & -0.811 & -0.492 & $\mathrm{~s}$ & 0.500 & 0.632 & 2.973 & -1.942 & 2 \\
\hline${ }^{85} \mathrm{Br}$ & 10.094 & 0.446 & -2.368 & $\mathrm{~s}$ & 3.716 & 0.822 & 3.847 & -1.804 & 9 \\
\hline${ }^{86} \mathrm{Br}$ & 10.218 & -0.762 & -2.500 & $\mathrm{~s}$ & 5.000 & 0.982 & 4.461 & -4.654 & 0 \\
\hline${ }^{87} \mathrm{Br}$ & 10.342 & -0.294 & -1.379 & $\mathrm{~s}$ & 3.300 & 0.810 & 3.943 & -2.789 & 0 \\
\hline${ }^{88} \mathrm{Br}$ & 10.466 & -0.702 & -0.343 & $\mathrm{~s}$ & 3.502 & 0.826 & 4.078 & -3.666 & 0 \\
\hline${ }^{89} \mathrm{Br}$ & 10.589 & 0.022 & 0.609 & $\mathrm{~s}$ & 4.207 & 0.800 & 4.057 & -2.974 & 0 \\
\hline${ }^{90} \mathrm{Br}$ & 10.713 & -0.527 & 1.476 & $\mathrm{~s}$ & 3.640 & 0.778 & 4.041 & -3.547 & 0 \\
\hline${ }^{77} \mathrm{Kr}$ & 9.106 & 0.065 & 6.284 & $\mathrm{~s}$ & 4.803 & 0.826 & 3.649 & -3.595 & 24 \\
\hline${ }^{78} \mathrm{Kr}$ & 9.229 & 2.017 & 6.238 & $\mathrm{~s}$ & 6.737 & 0.818 & 3.674 & -1.626 & 26 \\
\hline${ }^{79} \mathrm{Kr}$ & 9.352 & 0.018 & 5.397 & $\mathrm{x}$ & 5.431 & 0.872 & 3.861 & -4.177 & 26 \\
\hline${ }^{80} \mathrm{Kr}$ & 9.476 & 1.978 & 5.459 & $\mathrm{~s}$ & 3.000 & 0.517 & 2.549 & 1.050 & 23 \\
\hline${ }^{81} \mathrm{Kr}$ & 9.600 & 0.054 & 5.893 & $\mathrm{x}$ & 3.837 & 0.735 & 3.559 & -2.806 & 24 \\
\hline
\end{tabular}

Continued on next page... 
Table 10: (continued)

\begin{tabular}{|c|c|c|c|c|c|c|c|c|c|}
\hline Target & $\tilde{a}$ & $\Delta$ & $\delta W$ & $\mathrm{x} / \mathrm{s}$ & $E_{x}$ & $T$ & $\sigma\left(E_{x}\right)$ & $E_{0}$ & $N$ \\
\hline${ }^{82} \mathrm{Kr}$ & 9.723 & 1.908 & 3.765 & $\mathrm{~s}$ & 5.801 & 0.769 & 3.681 & -0.981 & 24 \\
\hline${ }^{83} \mathrm{Kr}$ & 9.847 & -0.085 & 2.575 & $\mathrm{~s}$ & 1.000 & 0.556 & 2.720 & -1.068 & 23 \\
\hline${ }^{84} \mathrm{Kr}$ & 9.970 & 1.771 & -1.076 & $\mathrm{x}$ & 6.629 & 0.911 & 4.150 & -1.610 & 25 \\
\hline${ }^{85} \mathrm{Kr}$ & 10.094 & -0.081 & 2.781 & $\mathrm{x}$ & 0.500 & 0.541 & 2.352 & -0.959 & 16 \\
\hline${ }^{86} \mathrm{Kr}$ & 10.218 & 1.916 & -2.368 & $\mathrm{~s}$ & 5.534 & 0.840 & 3.971 & -0.556 & 3 \\
\hline${ }^{87} \mathrm{Kr}$ & 10.342 & 0.408 & -2.500 & $\mathrm{~s}$ & 3.131 & 0.770 & 3.732 & -1.497 & 11 \\
\hline${ }^{88} \mathrm{Kr}$ & 10.466 & 1.441 & -1.379 & $\mathrm{~s}$ & 5.401 & 0.829 & 4.066 & -1.303 & 16 \\
\hline${ }^{89} \mathrm{Kr}$ & 10.589 & 0.538 & -0.343 & $\mathrm{~s}$ & 3.257 & 0.710 & 3.681 & -1.399 & 19 \\
\hline${ }^{90} \mathrm{Kr}$ & 10.713 & 1.381 & 0.609 & $\mathrm{~s}$ & 4.245 & 0.698 & 3.713 & -0.669 & 20 \\
\hline${ }^{91} \mathrm{Kr}$ & 10.837 & 0.524 & 1.476 & $\mathrm{~s}$ & 4.000 & 0.723 & 3.886 & -1.983 & 0 \\
\hline${ }^{77} \mathrm{Rb}$ & 9.106 & -0.077 & 6.101 & $\mathrm{~s}$ & 1.700 & 0.589 & 2.849 & -1.448 & 13 \\
\hline${ }^{78} \mathrm{Rb}$ & 9.229 & -0.882 & 6.284 & $\mathrm{~s}$ & 1.000 & 0.590 & 2.908 & -2.320 & 1 \\
\hline${ }^{79} \mathrm{Rb}$ & 9.352 & -0.096 & 6.238 & $\mathrm{~s}$ & 4.607 & 0.810 & 3.698 & -3.726 & 13 \\
\hline${ }^{80} \mathrm{Rb}$ & 9.476 & -0.884 & 5.963 & $\mathrm{~s}$ & 2.000 & 0.669 & 3.296 & -3.041 & 0 \\
\hline${ }^{81} \mathrm{Rb}$ & 9.600 & -0.111 & 5.459 & $\mathrm{~S}$ & 4.409 & 0.795 & 3.739 & -3.561 & 7 \\
\hline${ }^{82} \mathrm{Rb}$ & 9.723 & -1.067 & 4.726 & $\mathrm{~s}$ & 2.186 & 0.705 & 3.486 & -3.483 & 7 \\
\hline${ }^{83} \mathrm{Rb}$ & 9.847 & -0.218 & 3.765 & $\mathrm{~s}$ & 3.824 & 0.774 & 3.743 & -3.224 & 5 \\
\hline${ }^{84} \mathrm{Rb}$ & 9.970 & -0.832 & 2.575 & $\mathrm{~s}$ & 0.500 & 0.568 & 2.883 & -1.929 & 2 \\
\hline${ }^{85} \mathrm{Rb}$ & 10.094 & -0.196 & 1.156 & $\mathrm{~s}$ & 3.298 & 0.765 & 3.754 & -2.706 & 9 \\
\hline${ }^{86} \mathrm{Rb}$ & 10.218 & -0.534 & -1.622 & $\mathrm{x}$ & 2.520 & 0.782 & 3.773 & -2.667 & 10 \\
\hline${ }^{87} \mathrm{Rb}$ & 10.342 & 0.462 & -2.368 & $\mathrm{~s}$ & 4.131 & 0.837 & 4.011 & -2.043 & 8 \\
\hline${ }^{88} \mathrm{Rb}$ & 10.466 & -0.698 & -1.938 & $\mathrm{x}$ & 4.577 & 0.927 & 4.394 & -4.313 & 5 \\
\hline${ }^{89} \mathrm{Rb}$ & 10.589 & -0.189 & -1.379 & $\mathrm{~s}$ & 2.000 & 0.689 & 3.531 & -1.777 & 12 \\
\hline${ }^{90} \mathrm{Rb}$ & 10.713 & -0.596 & -0.343 & $\mathrm{~s}$ & 3.483 & 0.805 & 4.099 & -3.472 & 11 \\
\hline${ }^{91} \mathrm{Rb}$ & 10.837 & -0.247 & 0.609 & $\mathrm{~s}$ & 2.900 & 0.714 & 3.826 & -2.494 & 6 \\
\hline${ }^{92} \mathrm{Rb}$ & 10.961 & -0.667 & 1.476 & $\mathrm{~s}$ & 0.500 & 0.532 & 2.974 & -1.655 & 8 \\
\hline${ }^{93} \mathrm{Rb}$ & 11.085 & -0.120 & 2.260 & $\mathrm{~s}$ & 1.400 & 0.542 & 3.170 & -1.291 & 5 \\
\hline${ }^{94} \mathrm{Rb}$ & 11.209 & -0.848 & 2.959 & $\mathrm{~s}$ & 1.000 & 0.555 & 3.326 & -2.223 & 0 \\
\hline${ }^{95} \mathrm{Rb}$ & 11.333 & -0.111 & 3.574 & $\mathrm{~s}$ & 3.968 & 0.713 & 4.060 & -3.150 & 0 \\
\hline${ }^{78} \mathrm{Sr}$ & 9.229 & 1.559 & 6.101 & $\mathrm{~s}$ & 2.000 & 0.558 & 2.023 & 0.605 & 4 \\
\hline${ }^{79} \mathrm{Sr}$ & 9.352 & 0.068 & 6.284 & $\mathrm{~s}$ & 1.862 & 0.576 & 2.894 & -1.308 & 9 \\
\hline${ }^{80} \mathrm{Sr}$ & 9.476 & 1.852 & 6.238 & $\mathrm{~s}$ & 6.315 & 0.787 & 3.675 & -1.577 & 2 \\
\hline${ }^{81} \mathrm{Sr}$ & 9.600 & -0.143 & 5.963 & $\mathrm{~s}$ & 5.385 & 0.857 & 3.919 & -4.472 & 10 \\
\hline${ }^{82} \mathrm{Sr}$ & 9.723 & 1.982 & 5.459 & $\mathrm{~s}$ & 6.464 & 0.786 & 3.757 & -1.438 & 5 \\
\hline${ }^{83} \mathrm{Sr}$ & 9.847 & 0.046 & 4.726 & $\mathrm{~s}$ & 3.780 & 0.736 & 3.635 & -2.746 & 20 \\
\hline${ }^{84} \mathrm{Sr}$ & 9.970 & 1.940 & 3.765 & $\mathrm{~s}$ & 5.104 & 0.702 & 3.542 & -0.391 & 20 \\
\hline${ }^{85} \mathrm{Sr}$ & 10.094 & 0.052 & 2.678 & $\mathrm{x}$ & 2.099 & 0.621 & 3.230 & -1.473 & 39 \\
\hline${ }^{86} \mathrm{Sr}$ & 10.218 & 1.731 & 1.156 & $\mathrm{~s}$ & 3.535 & 0.622 & 3.202 & 0.362 & 41 \\
\hline${ }^{87} \mathrm{Sr}$ & 10.342 & 0.007 & -2.979 & $\mathrm{x}$ & 1.000 & 0.671 & 2.923 & -1.071 & 7 \\
\hline${ }^{88} \mathrm{Sr}$ & 10.466 & 2.074 & -3.517 & $\mathrm{x}$ & 3.000 & 0.685 & 2.913 & 0.986 & 35 \\
\hline${ }^{89} \mathrm{Sr}$ & 10.589 & 0.469 & -3.309 & $\mathrm{x}$ & 1.000 & 0.782 & 2.545 & -0.903 & 7 \\
\hline${ }^{90} \mathrm{Sr}$ & 10.713 & 1.464 & -1.379 & $\mathrm{~s}$ & 4.329 & 0.737 & 3.800 & -0.547 & 7 \\
\hline${ }^{91} \mathrm{Sr}$ & 10.837 & 0.399 & -0.343 & $\mathrm{~s}$ & 1.000 & 0.583 & 2.557 & -0.546 & 7 \\
\hline
\end{tabular}

Continued on next page... 
Table 10: (continued)

\begin{tabular}{|c|c|c|c|c|c|c|c|c|c|}
\hline Target & $\tilde{a}$ & $\Delta$ & $\delta W$ & $\mathrm{x} / \mathrm{s}$ & $E_{x}$ & $T$ & $\sigma\left(E_{x}\right)$ & $E_{0}$ & $N$ \\
\hline${ }^{92} \mathrm{Sr}$ & 10.961 & 1.443 & 0.609 & $\mathrm{~s}$ & 2.000 & 0.554 & 2.496 & 0.541 & 10 \\
\hline${ }^{93} \mathrm{Sr}$ & 11.085 & 0.377 & 1.476 & $\mathrm{~s}$ & 3.000 & 0.648 & 3.666 & -1.516 & 0 \\
\hline${ }^{94} \mathrm{Sr}$ & 11.209 & 1.584 & 2.260 & $\mathrm{~s}$ & 3.977 & 0.612 & 3.575 & -0.158 & 22 \\
\hline${ }^{95} \mathrm{Sr}$ & 11.333 & 0.447 & 2.959 & $\mathrm{~s}$ & 3.317 & 0.634 & 3.738 & -1.646 & 9 \\
\hline${ }^{96} \mathrm{Sr}$ & 11.458 & 1.435 & 3.574 & $\mathrm{~s}$ & 4.770 & 0.656 & 3.882 & -1.021 & 16 \\
\hline${ }^{97} \mathrm{Sr}$ & 11.582 & 0.469 & 4.105 & $\mathrm{~s}$ & 4.901 & 0.720 & 4.176 & -2.877 & 16 \\
\hline${ }^{98} \mathrm{Sr}$ & 11.706 & 1.763 & 4.552 & $\mathrm{~s}$ & 5.847 & 0.686 & 4.097 & -1.317 & 11 \\
\hline${ }^{80} \mathrm{Y}$ & 9.476 & -0.867 & 6.284 & $\mathrm{~s}$ & 3.508 & 0.780 & 3.655 & -4.225 & 0 \\
\hline${ }^{81} \mathrm{Y}$ & 9.600 & 0.040 & 6.238 & $\mathrm{~s}$ & 3.814 & 0.730 & 3.546 & -2.822 & 7 \\
\hline${ }^{82} \mathrm{Y}$ & 9.723 & -0.890 & 5.963 & $\mathrm{~s}$ & 0.500 & 0.528 & 2.780 & -1.999 & 10 \\
\hline${ }^{83} \mathrm{Y}$ & 9.847 & -0.136 & 5.459 & $\mathrm{~s}$ & 4.398 & 0.784 & 3.794 & -3.601 & 11 \\
\hline${ }^{84} \mathrm{Y}$ & 9.970 & -0.870 & 4.726 & $\mathrm{~s}$ & 1.000 & 0.579 & 3.073 & -2.284 & 7 \\
\hline${ }^{85} \mathrm{Y}$ & 10.094 & -0.130 & 3.765 & $\mathrm{~s}$ & 2.759 & 0.674 & 3.485 & -2.256 & 24 \\
\hline${ }^{86} \mathrm{Y}$ & 10.218 & -1.044 & 2.575 & $\mathrm{~s}$ & 1.000 & 0.617 & 3.254 & -2.566 & 6 \\
\hline${ }^{87} \mathrm{Y}$ & 10.342 & 0.086 & 1.156 & $\mathrm{~s}$ & 3.035 & 0.711 & 3.646 & -2.036 & 43 \\
\hline${ }^{88} \mathrm{Y}$ & 10.466 & -0.818 & -0.492 & $\mathrm{~s}$ & 1.000 & 0.646 & 3.313 & -2.193 & 9 \\
\hline${ }^{89} \mathrm{Y}$ & 10.589 & 0.833 & -2.368 & $\mathrm{~s}$ & 1.200 & 1.027 & 2.291 & -1.386 & 7 \\
\hline${ }^{90} \mathrm{Y}$ & 10.713 & -0.549 & -3.925 & $\mathrm{x}$ & 1.000 & 0.701 & 3.374 & -1.805 & 10 \\
\hline${ }^{91} \mathrm{Y}$ & 10.837 & -0.079 & -1.379 & $\mathrm{~s}$ & 1.000 & 0.596 & 2.998 & -1.089 & 9 \\
\hline${ }^{92} \mathrm{Y}$ & 10.961 & -0.519 & -0.343 & $\mathrm{~s}$ & 2.000 & 0.678 & 3.681 & -2.319 & 0 \\
\hline${ }^{93} \mathrm{Y}$ & 11.085 & -0.101 & 0.609 & $\mathrm{~s}$ & 1.000 & 0.539 & 2.978 & -1.065 & 3 \\
\hline${ }^{94} \mathrm{Y}$ & 11.209 & -0.445 & 1.476 & $\mathrm{~s}$ & 1.000 & 0.545 & 3.176 & -1.572 & 1 \\
\hline${ }^{95} \mathrm{Y}$ & 11.333 & -0.038 & 2.260 & $\mathrm{~s}$ & 0.500 & 0.491 & 2.473 & -0.833 & 3 \\
\hline${ }^{96} \mathrm{Y}$ & 11.458 & -0.535 & 2.959 & $\mathrm{~s}$ & 0.500 & 0.478 & 2.910 & -1.420 & 1 \\
\hline${ }^{97} \mathrm{Y}$ & 11.582 & -0.002 & 3.574 & $\mathrm{~s}$ & 0.500 & 0.454 & 2.427 & -0.738 & 2 \\
\hline${ }^{98} \mathrm{Y}$ & 11.706 & -0.786 & 4.105 & $\mathrm{~s}$ & 0.200 & 0.448 & 2.876 & -1.625 & 12 \\
\hline${ }^{99} \mathrm{Y}$ & 11.830 & -0.552 & 4.552 & $\mathrm{~s}$ & 0.300 & 0.428 & 2.776 & -1.318 & 2 \\
\hline${ }^{100} \mathrm{Y}$ & 11.954 & -0.395 & 4.914 & $\mathrm{~s}$ & 2.971 & 0.622 & 3.933 & -2.907 & 9 \\
\hline${ }^{101} \mathrm{Y}$ & 12.079 & -0.375 & 5.193 & $\mathrm{~s}$ & 0.000 & 0.428 & 2.271 & -1.090 & 8 \\
\hline${ }^{81} \mathrm{Zr}$ & 9.600 & 0.013 & 6.284 & $\mathrm{~s}$ & 4.365 & 0.772 & 3.676 & -3.326 & 0 \\
\hline${ }^{82} \mathrm{Zr}$ & 9.723 & 1.711 & 6.238 & $\mathrm{~s}$ & 5.666 & 0.737 & 3.614 & -1.299 & 2 \\
\hline${ }^{83} \mathrm{Zr}$ & 9.847 & 0.020 & 5.963 & $\mathrm{~s}$ & 6.140 & 0.882 & 4.079 & -4.835 & 4 \\
\hline${ }^{84} \mathrm{Zr}$ & 9.970 & 1.721 & 5.459 & $\mathrm{~s}$ & 6.007 & 0.760 & 3.766 & -1.539 & 1 \\
\hline${ }^{85} \mathrm{Zr}$ & 10.094 & -0.171 & 4.726 & $\mathrm{~s}$ & 4.094 & 0.763 & 3.812 & -3.389 & 0 \\
\hline${ }^{86} \mathrm{Zr}$ & 10.218 & 1.674 & 3.765 & $\mathrm{~s}$ & 3.000 & 0.536 & 2.884 & 0.601 & 12 \\
\hline${ }^{87} \mathrm{Zr}$ & 10.342 & 0.152 & 2.575 & $\mathrm{~s}$ & 2.675 & 0.652 & 3.454 & -1.693 & 14 \\
\hline${ }^{88} \mathrm{Zr}$ & 10.466 & 1.652 & 1.156 & $\mathrm{~s}$ & 4.308 & 0.682 & 3.575 & -0.267 & 25 \\
\hline${ }^{89} \mathrm{Zr}$ & 10.589 & 0.032 & -0.492 & $\mathrm{~s}$ & 1.000 & 0.581 & 2.849 & -0.934 & 1 \\
\hline${ }^{90} \mathrm{Zr}$ & 10.713 & 1.994 & -2.368 & $\mathrm{~s}$ & 4.300 & 0.716 & 3.645 & 0.346 & 10 \\
\hline${ }^{91} \mathrm{Zr}$ & 10.837 & 0.351 & -2.398 & $\mathrm{x}$ & 1.000 & 0.649 & 2.676 & -0.701 & 40 \\
\hline${ }^{92} \mathrm{Zr}$ & 10.961 & 1.314 & -1.743 & $\mathrm{x}$ & 4.658 & 0.769 & 4.016 & -0.999 & 5 \\
\hline${ }^{93} \mathrm{Zr}$ & 11.085 & 0.392 & -0.126 & $\mathrm{x}$ & 2.054 & 0.598 & 3.329 & -0.872 & 2 \\
\hline${ }^{94} \mathrm{Zr}$ & 11.209 & 1.322 & 2.169 & $\mathrm{x}$ & 3.058 & 0.558 & 3.301 & 0.020 & 8 \\
\hline
\end{tabular}

Continued on next page... 
Table 10: (continued)

\begin{tabular}{|c|c|c|c|c|c|c|c|c|c|}
\hline Target & $\tilde{a}$ & $\Delta$ & $\delta W$ & $\mathrm{x} / \mathrm{s}$ & $E_{x}$ & $T$ & $\sigma\left(E_{x}\right)$ & $E_{0}$ & $N$ \\
\hline${ }^{95} \mathrm{Zr}$ & 11.333 & 0.492 & 0.771 & $\mathrm{x}$ & 2.000 & 0.558 & 3.255 & -0.673 & 0 \\
\hline${ }^{96} \mathrm{Zr}$ & 11.458 & 1.589 & 2.260 & $\mathrm{~s}$ & 2.000 & 0.524 & 2.327 & 0.694 & 7 \\
\hline${ }^{97} \mathrm{Zr}$ & 11.582 & 0.795 & 0.130 & $\mathrm{x}$ & 2.000 & 0.536 & 3.138 & -0.205 & 9 \\
\hline${ }^{98} \mathrm{Zr}$ & 11.706 & 1.462 & 3.574 & $\mathrm{~s}$ & 4.487 & 0.624 & 3.832 & -0.757 & 9 \\
\hline${ }^{99} \mathrm{Zr}$ & 11.830 & 0.194 & 4.105 & $\mathrm{~s}$ & 3.976 & 0.666 & 4.058 & -2.629 & 20 \\
\hline${ }^{100} \mathrm{Zr}$ & 11.954 & 1.512 & 4.552 & $\mathrm{~s}$ & 6.154 & 0.713 & 4.282 & -2.032 & 7 \\
\hline${ }^{101} \mathrm{Zr}$ & 12.079 & 0.460 & 4.914 & $\mathrm{~s}$ & 4.623 & 0.673 & 4.175 & -2.701 & 8 \\
\hline${ }^{102} \mathrm{Zr}$ & 12.203 & 1.228 & 5.193 & $\mathrm{~s}$ & 5.484 & 0.671 & 4.212 & -2.022 & 2 \\
\hline${ }^{103} \mathrm{Zr}$ & 12.327 & 0.322 & 5.387 & $\mathrm{~s}$ & 2.456 & 0.511 & 3.549 & -1.251 & 6 \\
\hline${ }^{104} \mathrm{Zr}$ & 12.452 & 1.210 & 5.497 & $\mathrm{~S}$ & 3.120 & 0.488 & 3.467 & -0.201 & 4 \\
\hline${ }^{83} \mathrm{Nb}$ & 9.847 & 0.246 & 6.238 & $\mathrm{~s}$ & 4.411 & 0.747 & 3.688 & -2.939 & 2 \\
\hline${ }^{84} \mathrm{Nb}$ & 9.970 & -1.038 & 5.963 & $\mathrm{~s}$ & 1.000 & 0.576 & 3.105 & -2.569 & 0 \\
\hline${ }^{85} \mathrm{Nb}$ & 10.094 & 0.211 & 5.459 & $\mathrm{~s}$ & 4.475 & 0.753 & 3.787 & -3.033 & 0 \\
\hline${ }^{86} \mathrm{Nb}$ & 10.218 & -0.718 & 4.726 & $\mathrm{~s}$ & 3.526 & 0.756 & 3.833 & -3.920 & 0 \\
\hline${ }^{87} \mathrm{Nb}$ & 10.342 & 0.082 & 3.765 & $\mathrm{~s}$ & 1.000 & 0.500 & 2.647 & -0.785 & 7 \\
\hline${ }^{88} \mathrm{Nb}$ & 10.466 & -1.223 & 2.575 & $\mathrm{~s}$ & 1.000 & 0.622 & 3.368 & -2.860 & 1 \\
\hline${ }^{89} \mathrm{Nb}$ & 10.589 & 0.020 & 1.156 & $\mathrm{~s}$ & 1.000 & 0.543 & 2.803 & -0.909 & 6 \\
\hline${ }^{90} \mathrm{Nb}$ & 10.713 & -0.933 & -0.492 & $\mathrm{~s}$ & 1.000 & 0.644 & 3.408 & -2.367 & 11 \\
\hline${ }^{91} \mathrm{Nb}$ & 10.837 & 0.463 & -2.368 & $\mathrm{~s}$ & 3.992 & 0.801 & 4.076 & -1.949 & 13 \\
\hline${ }^{92} \mathrm{Nb}$ & 10.961 & -0.685 & -2.500 & $\mathrm{~s}$ & 4.631 & 0.914 & 4.546 & -4.287 & 7 \\
\hline${ }^{93} \mathrm{Nb}$ & 11.085 & -0.211 & -1.379 & $\mathrm{~s}$ & 2.570 & 0.713 & 3.843 & -2.163 & 6 \\
\hline${ }^{94} \mathrm{Nb}$ & 11.209 & -0.839 & 0.445 & $\mathrm{x}$ & 3.192 & 0.764 & 4.154 & -3.718 & 10 \\
\hline${ }^{95} \mathrm{Nb}$ & 11.333 & -0.225 & 0.609 & $\mathrm{~s}$ & 1.000 & 0.538 & 3.096 & -1.237 & 2 \\
\hline${ }^{96} \mathrm{Nb}$ & 11.458 & -0.793 & 1.476 & $\mathrm{~s}$ & 2.335 & 0.672 & 3.902 & -3.045 & 3 \\
\hline${ }^{97} \mathrm{Nb}$ & 11.582 & -0.066 & 2.260 & $\mathrm{~s}$ & 0.500 & 0.475 & 2.535 & -0.833 & 2 \\
\hline${ }^{98} \mathrm{Nb}$ & 11.706 & -0.683 & 2.959 & $\mathrm{~s}$ & 1.000 & 0.524 & 3.327 & -1.941 & 1 \\
\hline${ }^{99} \mathrm{Nb}$ & 11.830 & -0.408 & 3.574 & $\mathrm{~s}$ & 1.000 & 0.487 & 3.180 & -1.488 & 4 \\
\hline${ }^{100} \mathrm{Nb}$ & 11.954 & -0.698 & 4.105 & $\mathrm{~s}$ & 3.302 & 0.677 & 4.140 & -3.697 & 1 \\
\hline${ }^{101} \mathrm{Nb}$ & 12.079 & -0.170 & 4.552 & $\mathrm{~s}$ & 2.689 & 0.586 & 3.808 & -2.280 & 21 \\
\hline${ }^{102} \mathrm{Nb}$ & 12.203 & -0.793 & 4.914 & $\mathrm{~s}$ & 3.177 & 0.656 & 4.148 & -3.797 & 0 \\
\hline${ }^{103} \mathrm{Nb}$ & 12.327 & 0.157 & 5.193 & $\mathrm{~s}$ & 3.941 & 0.636 & 4.111 & -2.704 & 16 \\
\hline${ }^{104} \mathrm{Nb}$ & 12.452 & -0.746 & 5.387 & $\mathrm{~s}$ & 3.196 & 0.640 & 4.169 & -3.743 & 0 \\
\hline${ }^{105} \mathrm{Nb}$ & 12.576 & 0.374 & 5.497 & $\mathrm{~s}$ & 1.483 & 0.415 & 3.040 & -0.501 & 5 \\
\hline${ }^{84} \mathrm{Mo}$ & 9.970 & 2.437 & 6.238 & $\mathrm{~s}$ & 6.723 & 0.749 & 3.740 & -0.849 & 1 \\
\hline${ }^{85} \mathrm{Mo}$ & 10.094 & 0.241 & 5.963 & $\mathrm{~s}$ & 4.506 & 0.746 & 3.770 & -3.020 & 0 \\
\hline${ }^{86} \mathrm{Mo}$ & 10.218 & 1.289 & 5.459 & $\mathrm{~s}$ & 3.535 & 0.591 & 3.240 & -0.383 & 2 \\
\hline${ }^{87} \mathrm{Mo}$ & 10.342 & 0.247 & 4.726 & $\mathrm{~s}$ & 4.471 & 0.749 & 3.853 & -2.941 & 0 \\
\hline${ }^{88} \mathrm{Mo}$ & 10.466 & 1.519 & 3.765 & $\mathrm{~s}$ & 3.621 & 0.593 & 3.281 & -0.042 & 2 \\
\hline${ }^{89} \mathrm{Mo}$ & 10.589 & -0.033 & 2.575 & $\mathrm{~s}$ & 2.776 & 0.665 & 3.595 & -2.079 & 2 \\
\hline${ }^{90} \mathrm{Mo}$ & 10.713 & 1.707 & 1.156 & $\mathrm{~s}$ & 4.786 & 0.705 & 3.758 & -0.505 & 16 \\
\hline${ }^{91} \mathrm{Mo}$ & 10.837 & 0.063 & -0.492 & $\mathrm{~s}$ & 1.000 & 0.568 & 2.862 & -0.879 & 1 \\
\hline${ }^{92} \mathrm{Mo}$ & 10.961 & 1.994 & -2.368 & $\mathrm{~s}$ & 5.348 & 0.783 & 4.051 & -0.303 & 16 \\
\hline${ }^{93} \mathrm{Mo}$ & 11.085 & 0.349 & -2.758 & $\mathrm{x}$ & 1.000 & 0.646 & 2.725 & -0.695 & 18 \\
\hline
\end{tabular}

Continued on next page... 
Table 10: (continued)

\begin{tabular}{|c|c|c|c|c|c|c|c|c|c|}
\hline Target & $\tilde{\tilde{a}}$ & $\Delta$ & $\overline{\bar{c} \delta W}$ & $\mathrm{x} / \mathrm{s}$ & $E_{x}$ & $T$ & $\sigma\left(E_{x}\right)$ & $E_{0}$ & $N$ \\
\hline${ }^{94} \mathrm{Mo}$ & 11.209 & 1.476 & -1.379 & $\mathrm{~s}$ & 5.056 & 0.767 & 4.118 & -1.009 & 5 \\
\hline${ }^{95} \mathrm{Mo}$ & 11.333 & 0.362 & 0.300 & $\mathrm{x}$ & 4.394 & 0.761 & 4.186 & -2.512 & 8 \\
\hline${ }^{96} \mathrm{Mo}$ & 11.458 & 1.478 & 0.755 & $\mathrm{x}$ & 5.274 & 0.732 & 4.128 & -1.241 & 2 \\
\hline${ }^{97} \mathrm{Mo}$ & 11.582 & 0.307 & 1.833 & $\mathrm{x}$ & 3.944 & 0.698 & 4.062 & -2.332 & 5 \\
\hline${ }^{98} \mathrm{Mo}$ & 11.706 & 1.503 & 2.627 & $\mathrm{x}$ & 4.679 & 0.648 & 3.917 & -0.811 & 5 \\
\hline${ }^{99} \mathrm{Mo}$ & 11.830 & 0.006 & 3.376 & $\mathrm{x}$ & 3.545 & 0.660 & 4.019 & -2.605 & 12 \\
\hline${ }^{100} \mathrm{Mo}$ & 11.954 & 1.649 & 3.574 & $\mathrm{~s}$ & 5.822 & 0.696 & 4.206 & -1.470 & 14 \\
\hline${ }^{101} \mathrm{Mo}$ & 12.079 & -0.050 & 5.249 & $\mathrm{x}$ & 4.066 & 0.666 & 4.150 & -3.184 & 5 \\
\hline${ }^{102} \mathrm{Mo}$ & 12.203 & 1.583 & 4.552 & $\mathrm{~s}$ & 5.965 & 0.688 & 4.268 & -1.747 & 7 \\
\hline${ }^{103} \mathrm{Mo}$ & 12.327 & 0.167 & 4.914 & $\mathrm{~s}$ & 3.555 & 0.612 & 4.007 & -2.365 & 16 \\
\hline${ }^{104} \mathrm{Mo}$ & 12.452 & 1.381 & 5.193 & $\mathrm{~s}$ & 5.332 & 0.643 & 4.179 & -1.617 & 16 \\
\hline${ }^{105} \mathrm{Mo}$ & 12.576 & 0.192 & 5.387 & $\mathrm{~s}$ & 3.313 & 0.579 & 3.951 & -2.138 & 2 \\
\hline${ }^{106} \mathrm{Mo}$ & 12.700 & 1.196 & 5.497 & $\mathrm{~s}$ & 4.955 & 0.619 & 4.160 & -1.654 & 6 \\
\hline${ }^{107} \mathrm{Mo}$ & 12.825 & 0.291 & 5.522 & $\mathrm{~s}$ & 4.193 & 0.624 & 4.222 & -2.680 & 0 \\
\hline${ }^{87} \mathrm{Tc}$ & 10.342 & 0.092 & 5.459 & $\mathrm{~s}$ & 1.000 & 0.472 & 2.596 & -0.743 & 0 \\
\hline${ }^{88} \mathrm{Tc}$ & 10.466 & -0.834 & 4.726 & $\mathrm{~s}$ & 3.371 & 0.742 & 3.874 & -4.006 & 0 \\
\hline${ }^{89} \mathrm{Tc}$ & 10.589 & -0.137 & 3.765 & $\mathrm{~s}$ & 4.048 & 0.749 & 3.930 & -3.261 & 0 \\
\hline${ }^{90} \mathrm{Tc}$ & 10.713 & -0.666 & 2.575 & $\mathrm{~s}$ & 3.501 & 0.760 & 3.996 & -3.731 & 0 \\
\hline${ }^{91} \mathrm{Tc}$ & 10.837 & -0.195 & 1.156 & $\mathrm{~s}$ & 2.350 & 0.657 & 3.606 & -2.033 & 2 \\
\hline${ }^{92} \mathrm{Tc}$ & 10.961 & -0.829 & -0.492 & $\mathrm{~s}$ & 0.500 & 0.584 & 3.143 & -1.923 & 8 \\
\hline${ }^{93} \mathrm{Tc}$ & 11.085 & 0.487 & -2.368 & $\mathrm{~s}$ & 3.752 & 0.770 & 4.049 & -1.752 & 17 \\
\hline${ }^{94} \mathrm{Tc}$ & 11.209 & -0.814 & -2.500 & $\mathrm{~s}$ & 3.282 & 0.825 & 4.316 & -3.593 & 1 \\
\hline${ }^{95} \mathrm{Tc}$ & 11.333 & -0.096 & -1.379 & $\mathrm{~s}$ & 3.781 & 0.782 & 4.225 & -2.785 & 8 \\
\hline${ }^{96} \mathrm{Tc}$ & 11.458 & -0.947 & -0.343 & $\mathrm{~s}$ & 5.000 & 0.886 & 4.674 & -5.209 & 1 \\
\hline${ }^{97} \mathrm{Tc}$ & 11.582 & -0.095 & 0.609 & $\mathrm{~s}$ & 3.789 & 0.736 & 4.183 & -2.874 & 10 \\
\hline${ }^{98} \mathrm{Tc}$ & 11.706 & -0.997 & 1.476 & $\mathrm{~s}$ & 4.226 & 0.803 & 4.492 & -4.847 & 7 \\
\hline${ }^{99} \mathrm{Tc}$ & 11.830 & -0.121 & 2.260 & $\mathrm{~s}$ & 2.895 & 0.638 & 3.903 & -2.307 & 5 \\
\hline${ }^{100} \mathrm{Tc}$ & 11.954 & -0.877 & 5.180 & $\mathrm{x}$ & 1.877 & 0.574 & 3.729 & -2.916 & 24 \\
\hline${ }^{101} \mathrm{Tc}$ & 12.079 & -0.130 & 3.574 & $\mathrm{~s}$ & 3.448 & 0.651 & 4.068 & -2.778 & 23 \\
\hline${ }^{102} \mathrm{Tc}$ & 12.203 & -0.860 & 4.105 & $\mathrm{~s}$ & 2.000 & 0.588 & 3.846 & -2.964 & 1 \\
\hline${ }^{103} \mathrm{Tc}$ & 12.327 & -0.077 & 4.552 & $\mathrm{~s}$ & 3.906 & 0.657 & 4.189 & -3.081 & 5 \\
\hline${ }^{104} \mathrm{Tc}$ & 12.452 & -0.929 & 4.914 & $\mathrm{~s}$ & 1.000 & 0.496 & 3.494 & -2.350 & 1 \\
\hline${ }^{105} \mathrm{Tc}$ & 12.576 & 0.060 & 5.193 & $\mathrm{~s}$ & 4.307 & 0.658 & 4.280 & -3.187 & 18 \\
\hline${ }^{106} \mathrm{Tc}$ & 12.700 & -0.888 & 5.387 & $\mathrm{~s}$ & 2.000 & 0.559 & 3.895 & -3.034 & 0 \\
\hline${ }^{107} \mathrm{Tc}$ & 12.825 & 0.189 & 5.497 & $\mathrm{~s}$ & 4.091 & 0.624 & 4.223 & -2.781 & 0 \\
\hline${ }^{108} \mathrm{Tc}$ & 12.949 & -0.905 & 5.522 & $\mathrm{~s}$ & 2.984 & 0.620 & 4.241 & -3.867 & 0 \\
\hline${ }^{88} \mathrm{Ru}$ & 10.466 & 2.239 & 5.459 & $\mathrm{~s}$ & 6.444 & 0.732 & 3.849 & -0.958 & 0 \\
\hline${ }^{89} \mathrm{Ru}$ & 10.589 & 0.293 & 4.726 & $\mathrm{~s}$ & 4.478 & 0.735 & 3.895 & -2.865 & 0 \\
\hline${ }^{90} \mathrm{Ru}$ & 10.713 & 1.527 & 3.765 & $\mathrm{~s}$ & 5.694 & 0.742 & 3.951 & -1.583 & 0 \\
\hline${ }^{91} \mathrm{Ru}$ & 10.837 & 0.088 & 2.575 & $\mathrm{~s}$ & 0.600 & 0.515 & 2.376 & -0.755 & 4 \\
\hline${ }^{92} \mathrm{Ru}$ & 10.961 & 1.638 & 1.156 & $\mathrm{~s}$ & 4.000 & 0.637 & 3.561 & -0.075 & 2 \\
\hline${ }^{93} \mathrm{Ru}$ & 11.085 & 0.053 & -0.492 & $\mathrm{~s}$ & 0.500 & 0.639 & 2.408 & -1.064 & 4 \\
\hline${ }^{94} \mathrm{Ru}$ & 11.209 & 1.904 & -2.368 & $\mathrm{~s}$ & 4.557 & 0.719 & 3.869 & 0.054 & 3 \\
\hline
\end{tabular}

Continued on next page... 
Table 10: (continued)

\begin{tabular}{|c|c|c|c|c|c|c|c|c|c|}
\hline Target & $\tilde{\tilde{a}}$ & $\Delta$ & $\delta W$ & $\mathrm{x} / \mathrm{s}$ & $E_{x}$ & $T$ & $\sigma\left(E_{x}\right)$ & $E_{0}$ & $N$ \\
\hline${ }^{95} \mathrm{Ru}$ & 11.333 & 0.331 & -2.500 & $\mathrm{~s}$ & 3.000 & 0.718 & 3.905 & -1.524 & 1 \\
\hline${ }^{96} \mathrm{Ru}$ & 11.458 & 1.530 & -1.379 & $\mathrm{~s}$ & 5.998 & 0.815 & 4.403 & -1.572 & 27 \\
\hline${ }^{97} \mathrm{Ru}$ & 11.582 & 0.290 & -0.343 & $\mathrm{~s}$ & 4.000 & 0.740 & 4.179 & -2.325 & 12 \\
\hline${ }^{98} \mathrm{Ru}$ & 11.706 & 1.594 & 0.609 & $\mathrm{~s}$ & 5.939 & 0.761 & 4.328 & -1.530 & 13 \\
\hline${ }^{99} \mathrm{Ru}$ & 11.830 & 0.253 & 1.476 & $\mathrm{~s}$ & 4.250 & 0.719 & 4.224 & -2.647 & 23 \\
\hline${ }^{100} \mathrm{Ru}$ & 11.954 & 1.645 & 2.625 & $\mathrm{x}$ & 5.666 & 0.699 & 4.205 & -1.320 & 12 \\
\hline${ }^{101} \mathrm{Ru}$ & 12.079 & 0.173 & 2.959 & $\mathrm{~s}$ & 4.374 & 0.702 & 4.262 & -2.947 & 7 \\
\hline${ }^{102} \mathrm{Ru}$ & 12.203 & 1.706 & 4.441 & $\mathrm{x}$ & 5.572 & 0.655 & 4.138 & -1.197 & 13 \\
\hline${ }^{103} \mathrm{Ru}$ & 12.327 & 0.083 & 3.471 & $\mathrm{x}$ & 4.990 & 0.730 & 4.461 & -3.636 & 5 \\
\hline${ }^{104} \mathrm{Ru}$ & 12.452 & 1.682 & 4.552 & $\mathrm{~s}$ & 4.293 & 0.555 & 3.785 & -0.239 & 26 \\
\hline${ }^{105} \mathrm{Ru}$ & 12.576 & 0.113 & 5.983 & $\mathrm{x}$ & 4.560 & 0.662 & 4.300 & -3.331 & 4 \\
\hline${ }^{106} \mathrm{Ru}$ & 12.700 & 1.572 & 5.193 & $\mathrm{~s}$ & 5.499 & 0.634 & 4.218 & -1.410 & 7 \\
\hline${ }^{107} \mathrm{Ru}$ & 12.825 & 0.225 & 5.387 & $\mathrm{~s}$ & 5.000 & 0.681 & 4.450 & -3.479 & 0 \\
\hline${ }^{108} \mathrm{Ru}$ & 12.949 & 1.451 & 5.497 & $\mathrm{~s}$ & 5.673 & 0.641 & 4.331 & -1.789 & 9 \\
\hline${ }^{109} \mathrm{Ru}$ & 13.074 & 0.255 & 5.522 & $\mathrm{~s}$ & 4.784 & 0.657 & 4.432 & -3.249 & 2 \\
\hline${ }^{91} \mathrm{Rh}$ & 10.837 & -0.242 & 3.765 & $\mathrm{~s}$ & 3.906 & 0.735 & 3.971 & -3.339 & 0 \\
\hline${ }^{92} \mathrm{Rh}$ & 10.961 & -0.798 & 2.575 & $\mathrm{~s}$ & 3.332 & 0.747 & 4.038 & -3.837 & 0 \\
\hline${ }^{93} \mathrm{Rh}$ & 11.085 & -0.091 & 1.156 & $\mathrm{~s}$ & 1.000 & 0.528 & 2.953 & -1.042 & 0 \\
\hline${ }^{94} \mathrm{Rh}$ & 11.209 & -0.794 & -0.492 & $\mathrm{~s}$ & 0.500 & 0.571 & 3.161 & -1.861 & 1 \\
\hline${ }^{95} \mathrm{Rh}$ & 11.333 & 0.486 & -2.368 & $\mathrm{~s}$ & 1.000 & 0.663 & 2.587 & -0.641 & 2 \\
\hline${ }^{96} \mathrm{Rh}$ & 11.458 & -0.865 & -2.500 & $\mathrm{~s}$ & 4.600 & 0.897 & 4.690 & -4.577 & 3 \\
\hline${ }^{97} \mathrm{Rh}$ & 11.582 & 0.033 & -1.379 & $\mathrm{~s}$ & 3.514 & 0.743 & 4.163 & -2.384 & 19 \\
\hline${ }^{98} \mathrm{Rh}$ & 11.706 & -0.941 & -0.343 & $\mathrm{~s}$ & 3.090 & 0.757 & 4.292 & -3.787 & 1 \\
\hline${ }^{99} \mathrm{Rh}$ & 11.830 & 0.022 & 0.609 & $\mathrm{~s}$ & 2.957 & 0.658 & 3.946 & -2.072 & 29 \\
\hline${ }^{100} \mathrm{Rh}$ & 11.954 & -0.923 & 1.476 & $\mathrm{~s}$ & 2.852 & 0.699 & 4.187 & -3.655 & 2 \\
\hline${ }^{101} \mathrm{Rh}$ & 12.079 & 0.032 & 2.260 & $\mathrm{~s}$ & 3.293 & 0.647 & 4.026 & -2.337 & 13 \\
\hline${ }^{102} \mathrm{Rh}$ & 12.203 & -0.940 & 2.959 & $\mathrm{~s}$ & 3.182 & 0.692 & 4.266 & -3.998 & 22 \\
\hline${ }^{103} \mathrm{Rh}$ & 12.327 & -0.031 & 3.574 & $\mathrm{~s}$ & 2.180 & 0.540 & 3.644 & -1.647 & 37 \\
\hline${ }^{104} \mathrm{Rh}$ & 12.452 & -0.935 & 3.746 & $\mathrm{x}$ & 2.404 & 0.619 & 4.059 & -3.402 & 22 \\
\hline${ }^{105} \mathrm{Rh}$ & 12.576 & 0.020 & 4.552 & $\mathrm{~s}$ & 2.844 & 0.568 & 3.882 & -2.063 & 5 \\
\hline${ }^{106} \mathrm{Rh}$ & 12.700 & -0.890 & 4.914 & $\mathrm{~s}$ & 3.026 & 0.636 & 4.226 & -3.852 & 1 \\
\hline${ }^{107} \mathrm{Rh}$ & 12.825 & 0.056 & 5.193 & $\mathrm{~s}$ & 3.388 & 0.589 & 4.068 & -2.439 & 10 \\
\hline${ }^{108} \mathrm{Rh}$ & 12.949 & -0.831 & 5.387 & $\mathrm{~s}$ & 3.057 & 0.621 & 4.246 & -3.789 & 0 \\
\hline${ }^{109} \mathrm{Rh}$ & 13.074 & 0.056 & 5.497 & $\mathrm{~s}$ & 2.420 & 0.508 & 3.758 & -1.687 & 26 \\
\hline${ }^{110} \mathrm{Rh}$ & 13.198 & -0.780 & 5.522 & $\mathrm{~s}$ & 1.000 & 0.458 & 3.516 & -2.092 & 0 \\
\hline${ }^{94} \mathrm{Pd}$ & 11.209 & 1.569 & 1.156 & $\mathrm{~s}$ & 5.665 & 0.756 & 4.139 & -1.388 & 0 \\
\hline${ }^{95} \mathrm{Pd}$ & 11.333 & 0.129 & -0.492 & $\mathrm{~s}$ & 4.208 & 0.779 & 4.236 & -2.743 & 0 \\
\hline${ }^{96} \mathrm{Pd}$ & 11.458 & 1.952 & -2.368 & $\mathrm{~s}$ & 5.262 & 0.757 & 4.137 & -0.315 & 4 \\
\hline${ }^{97} \mathrm{Pd}$ & 11.582 & 0.232 & -2.500 & $\mathrm{~s}$ & 1.000 & 0.588 & 2.899 & -0.708 & 2 \\
\hline${ }^{98} \mathrm{Pd}$ & 11.706 & 1.466 & -1.379 & $\mathrm{~s}$ & 3.446 & 0.624 & 3.639 & 0.028 & 3 \\
\hline${ }^{99} \mathrm{Pd}$ & 11.830 & 0.225 & -0.343 & $\mathrm{~s}$ & 4.576 & 0.773 & 4.400 & -2.854 & 3 \\
\hline${ }^{100} \mathrm{Pd}$ & 11.954 & 1.623 & 0.609 & $\mathrm{~s}$ & 5.155 & 0.697 & 4.157 & -0.901 & 10 \\
\hline${ }^{101} \mathrm{Pd}$ & 12.079 & 0.178 & 1.476 & $\mathrm{~s}$ & 2.000 & 0.546 & 3.508 & -1.164 & 18 \\
\hline
\end{tabular}

Continued on next page... 
Table 10: (continued)

\begin{tabular}{lcccccccrr}
\hline \hline Target & $\tilde{a}$ & $\Delta$ & $\delta W$ & $\mathrm{x} / \mathrm{s}$ & $E_{x}$ & $T$ & $\sigma\left(E_{x}\right)$ & $E_{0}$ & $N$ \\
\hline${ }^{102} \mathrm{Pd}$ & 12.203 & 1.648 & 2.260 & $\mathrm{~s}$ & 4.912 & 0.643 & 4.050 & -0.723 & 3 \\
${ }^{103} \mathrm{Pd}$ & 12.327 & 0.179 & 2.959 & $\mathrm{~s}$ & 3.983 & 0.666 & 4.204 & -2.629 & 6 \\
${ }^{104} \mathrm{Pd}$ & 12.452 & 1.645 & 3.574 & $\mathrm{~s}$ & 5.099 & 0.630 & 4.101 & -0.908 & 7 \\
${ }^{105} \mathrm{Pd}$ & 12.576 & 0.118 & 2.765 & $\mathrm{x}$ & 3.851 & 0.656 & 4.238 & -2.630 & 5 \\
${ }^{106} \mathrm{Pd}$ & 12.700 & 1.645 & 3.824 & $\mathrm{x}$ & 5.307 & 0.633 & 4.197 & -1.079 & 26 \\
${ }^{107} \mathrm{Pd}$ & 12.825 & 0.020 & 3.413 & $\mathrm{x}$ & 4.288 & 0.674 & 4.404 & -3.180 & 8 \\
${ }^{108} \mathrm{Pd}$ & 12.949 & 1.634 & 4.142 & $\mathrm{x}$ & 5.436 & 0.631 & 4.270 & -1.215 & 10 \\
${ }^{109} \mathrm{Pd}$ & 13.074 & 0.018 & 5.329 & $\mathrm{x}$ & 2.912 & 0.550 & 3.962 & -2.132 & 2 \\
& & & & & & & & & \\
\hline${ }^{102} \mathrm{Ag}$ & 12.203 & -0.758 & 1.476 & $\mathrm{~s}$ & 1.400 & 0.570 & 3.681 & -2.322 & 13 \\
${ }^{103} \mathrm{Ag}$ & 12.327 & -0.028 & 2.260 & $\mathrm{~s}$ & 1.000 & 0.459 & 3.047 & -0.890 & 9 \\
${ }^{104} \mathrm{Ag}$ & 12.452 & -0.806 & 2.959 & $\mathrm{~s}$ & 2.230 & 0.608 & 3.993 & -3.022 & 6 \\
${ }^{105} \mathrm{Ag}$ & 12.576 & -0.075 & 3.574 & $\mathrm{~s}$ & 1.981 & 0.520 & 3.617 & -1.579 & 6 \\
${ }^{106} \mathrm{Ag}$ & 12.700 & -0.779 & 4.105 & $\mathrm{~s}$ & 0.200 & 0.418 & 3.003 & -1.588 & 4 \\
${ }^{107} \mathrm{Ag}$ & 12.825 & -0.022 & 4.552 & $\mathrm{~s}$ & 0.752 & 0.394 & 2.833 & -0.721 & 10 \\
${ }^{97} \mathrm{Ag}$ & 11.582 & 0.446 & -2.368 & $\mathrm{~s}$ & 0.700 & 2.226 & 2.195 & -6.523 & 4 \\
${ }^{98} \mathrm{Ag}$ & 11.706 & -0.747 & -2.500 & $\mathrm{~s}$ & 3.284 & 0.798 & 4.403 & -3.485 & 0 \\
${ }^{99} \mathrm{Ag}$ & 11.830 & 0.068 & -1.379 & $\mathrm{~s}$ & 1.641 & 0.586 & 3.456 & -1.132 & 3 \\
${ }^{100} \mathrm{Ag}$ & 11.954 & -0.831 & -0.343 & $\mathrm{~s}$ & 4.443 & 0.825 & 4.643 & -4.596 & 2 \\
${ }^{101} \mathrm{Ag}$ & 12.079 & -0.105 & 0.609 & $\mathrm{~s}$ & 1.000 & 0.502 & 3.125 & -1.034 & 14 \\
${ }^{108} \mathrm{Ag}$ & 12.949 & -0.898 & 3.188 & $\mathrm{x}$ & 1.804 & 0.565 & 3.954 & -2.868 & 19 \\
${ }^{109} \mathrm{Ag}$ & 13.074 & -0.012 & 5.193 & $\mathrm{~s}$ & 2.321 & 0.509 & 3.757 & -1.730 & 4 \\
${ }^{110} \mathrm{Ag}$ & 13.198 & -0.991 & 4.695 & $\mathrm{x}$ & 1.000 & 0.484 & 3.645 & -2.452 & 0 \\
& & & & & & & & & \\
\hline
\end{tabular}




\section{Modeled Cross Sections Compared to Measurements}

C.1. $(\mathrm{n}, \gamma)$
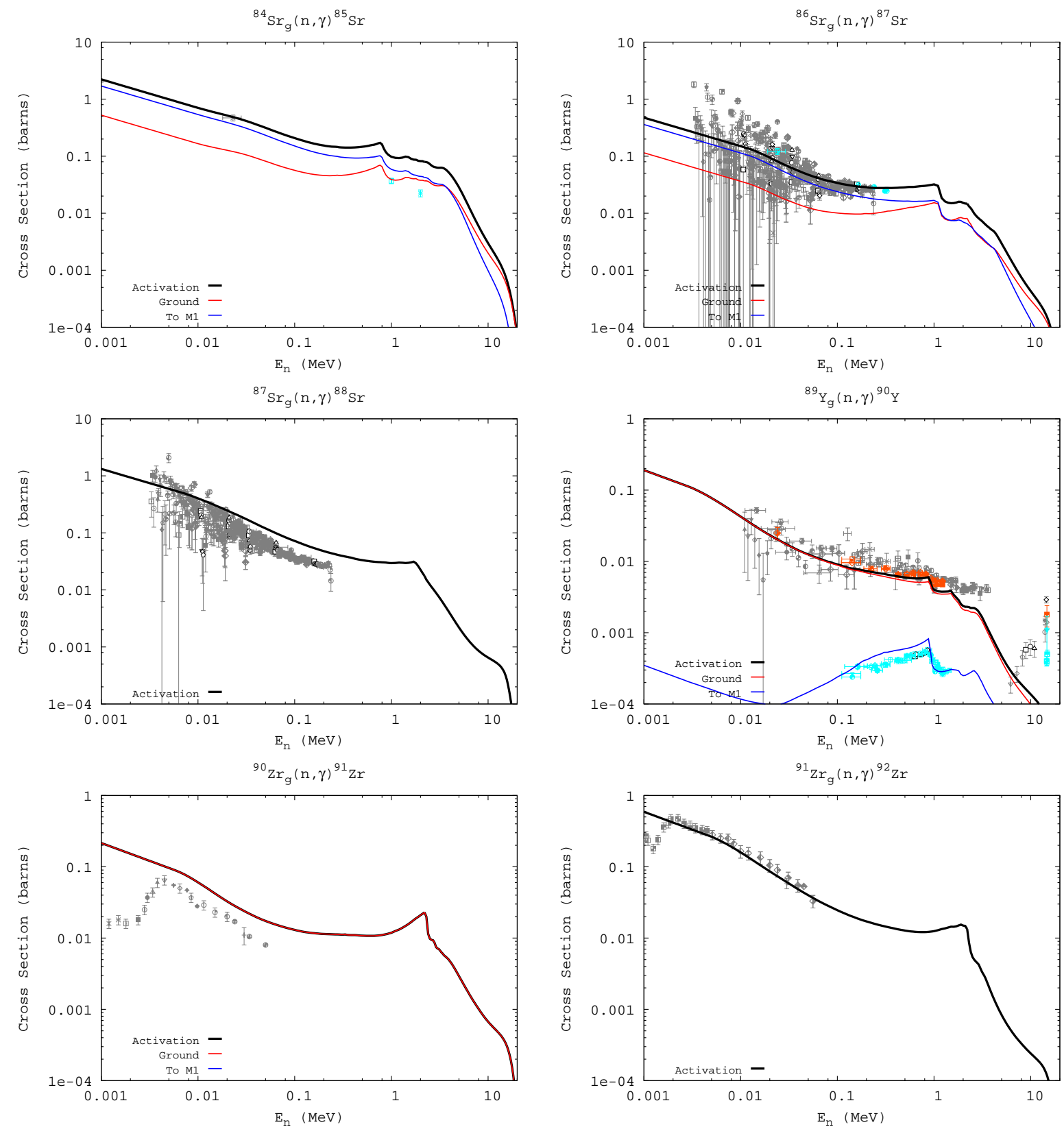

Fig. 25.- Modeled neutron capture cross sections compared to measurement. The data is taken from (EXFOR 2006). The black, red, and blue solid lines represent our modeled cross sections (total, leading to the ground state, and leading to the first isomer, respectively). The gray, orange, and light blue data points are measured cross section data (total, ground state, and first isomer). 

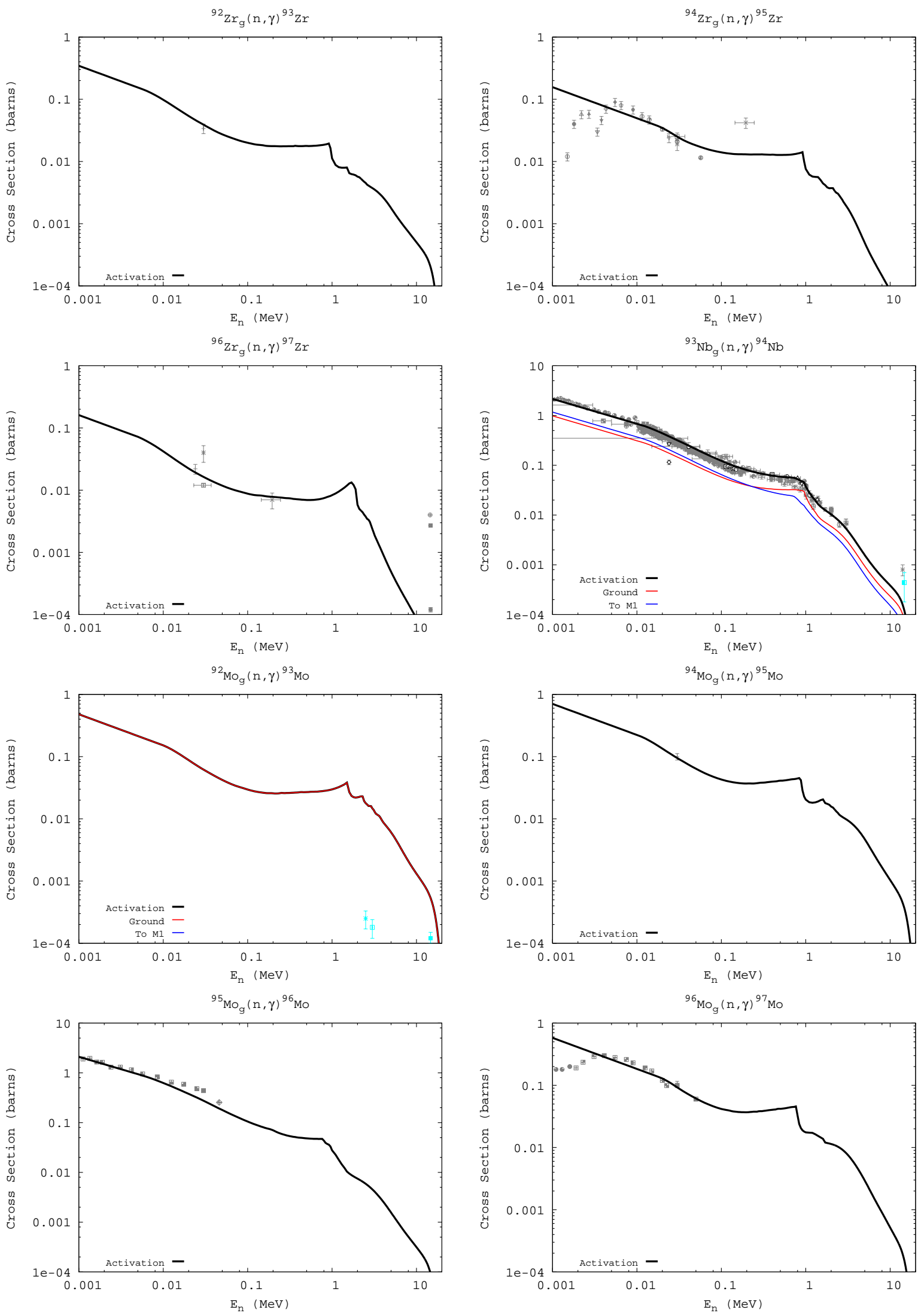

Fig. 25.- (continued) 

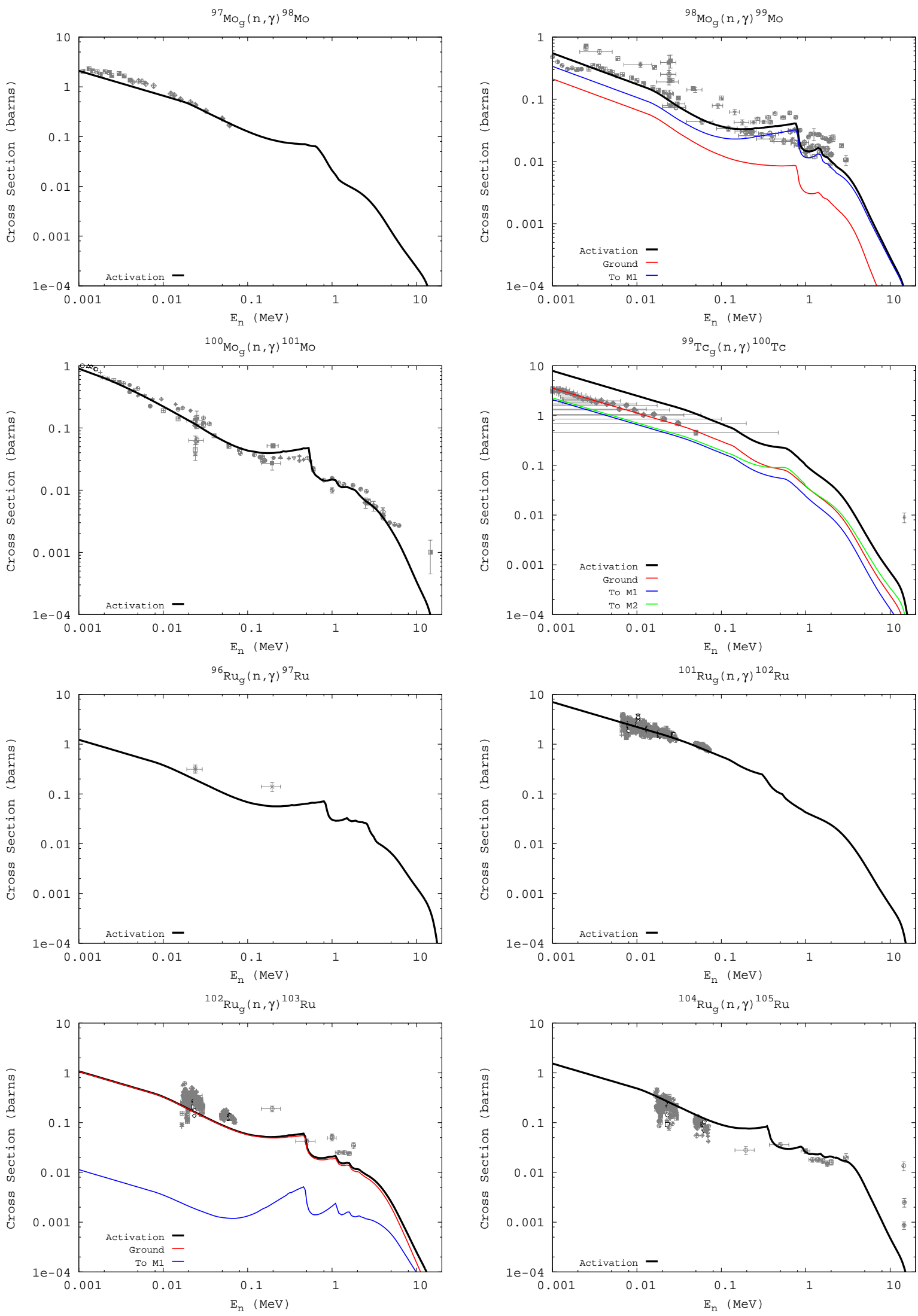

Fig. 25.- (continued) 


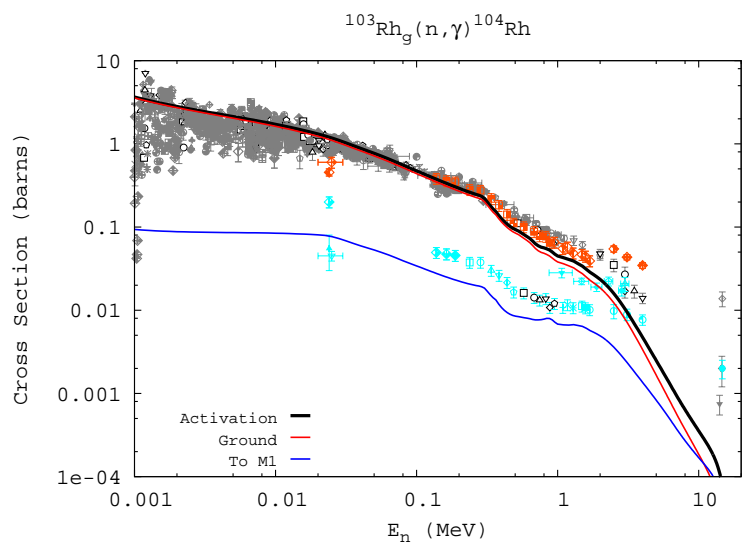

Fig. 25.- (continued) 


\section{C.2. Maxwellian-averaged $(\mathrm{n}, \gamma)$}
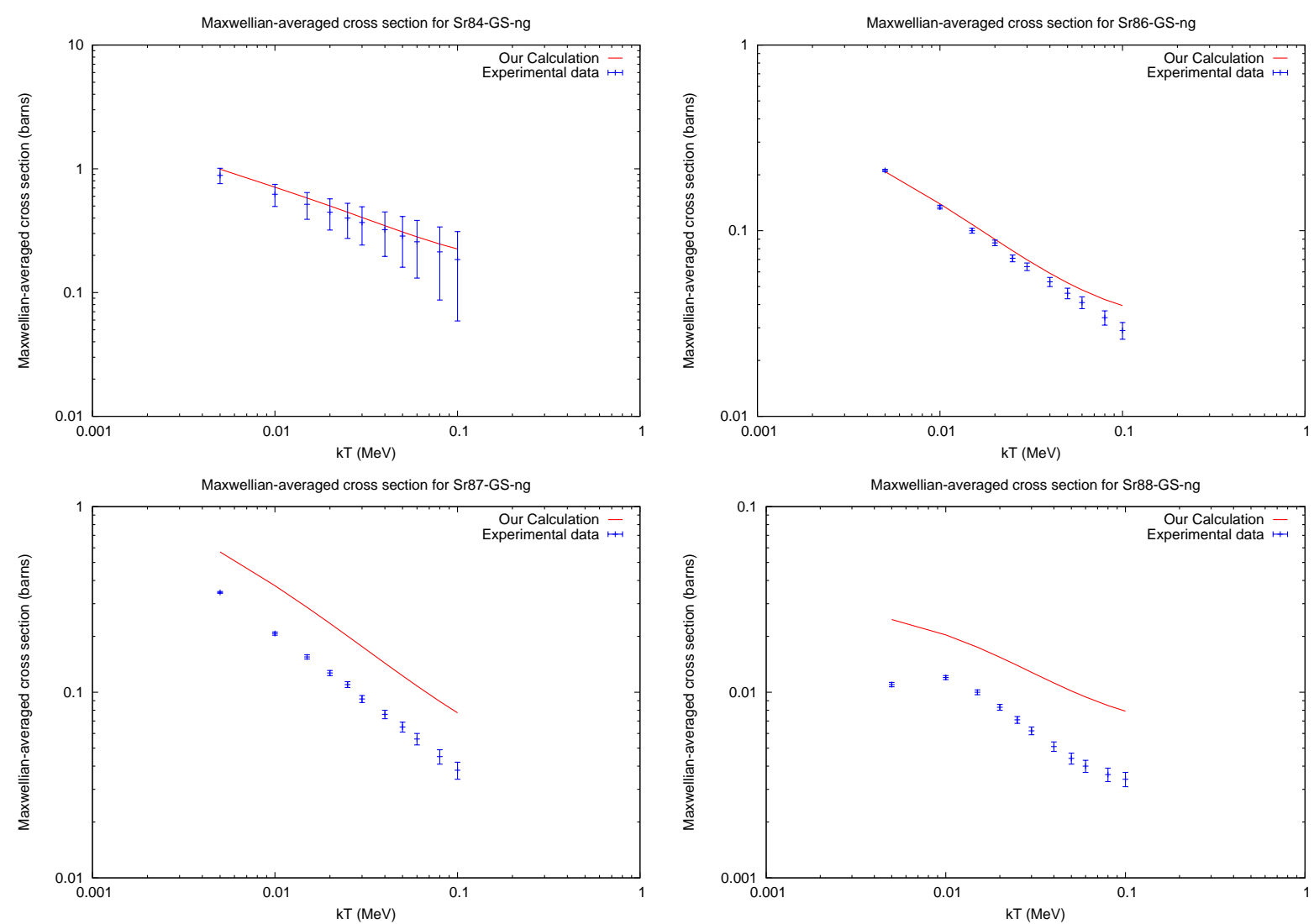

Fig. 26.- Modeled Maxwellian-averaged neutron capture cross sections compared to measurement. The data is taken from (Bao et al. 2000). The solid lines represent our modeled cross sections. The errors on the recommended value for each energy are identical to the stated error at $30 \mathrm{keV}$. 

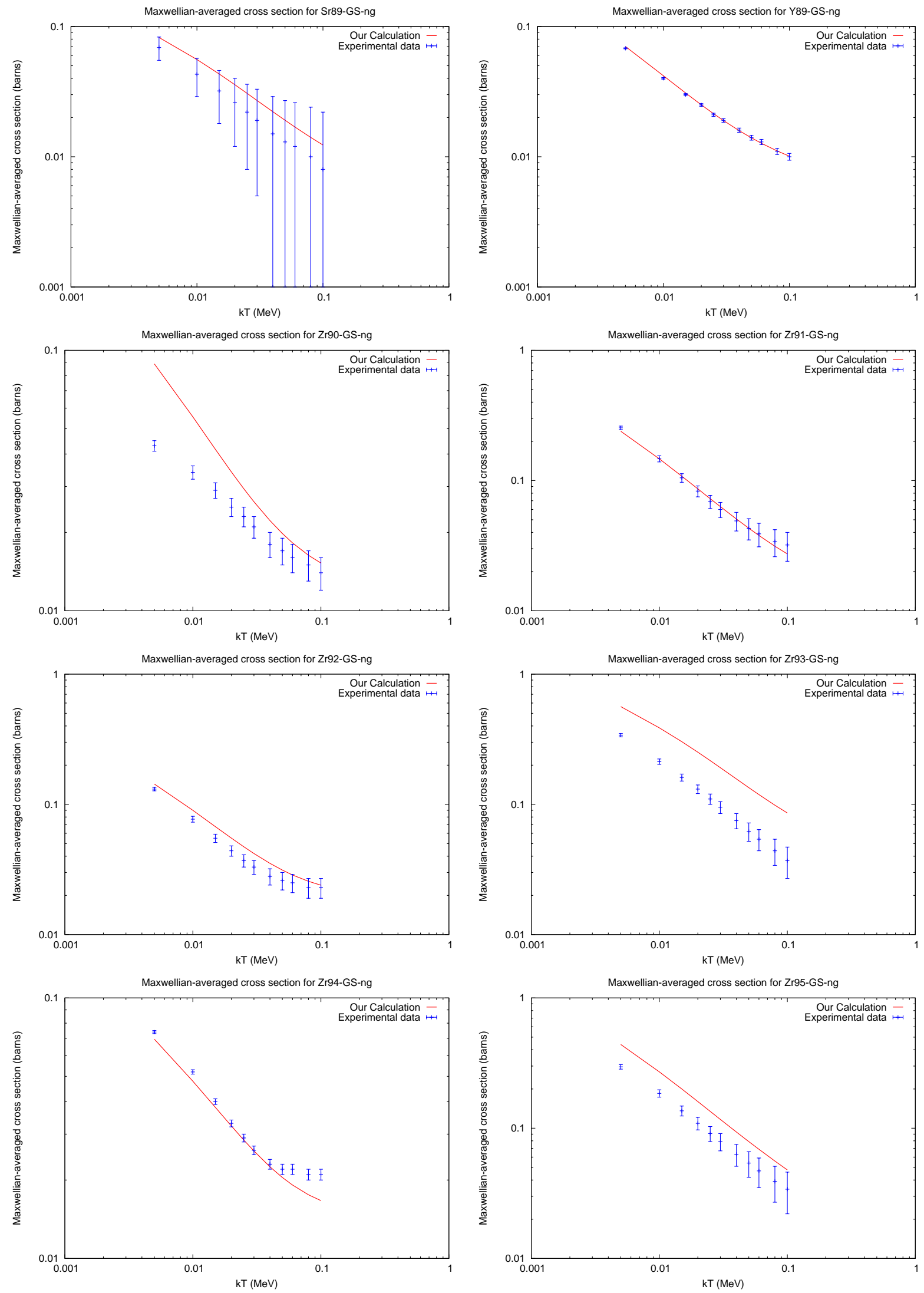

Fig. 26.- (continued) 

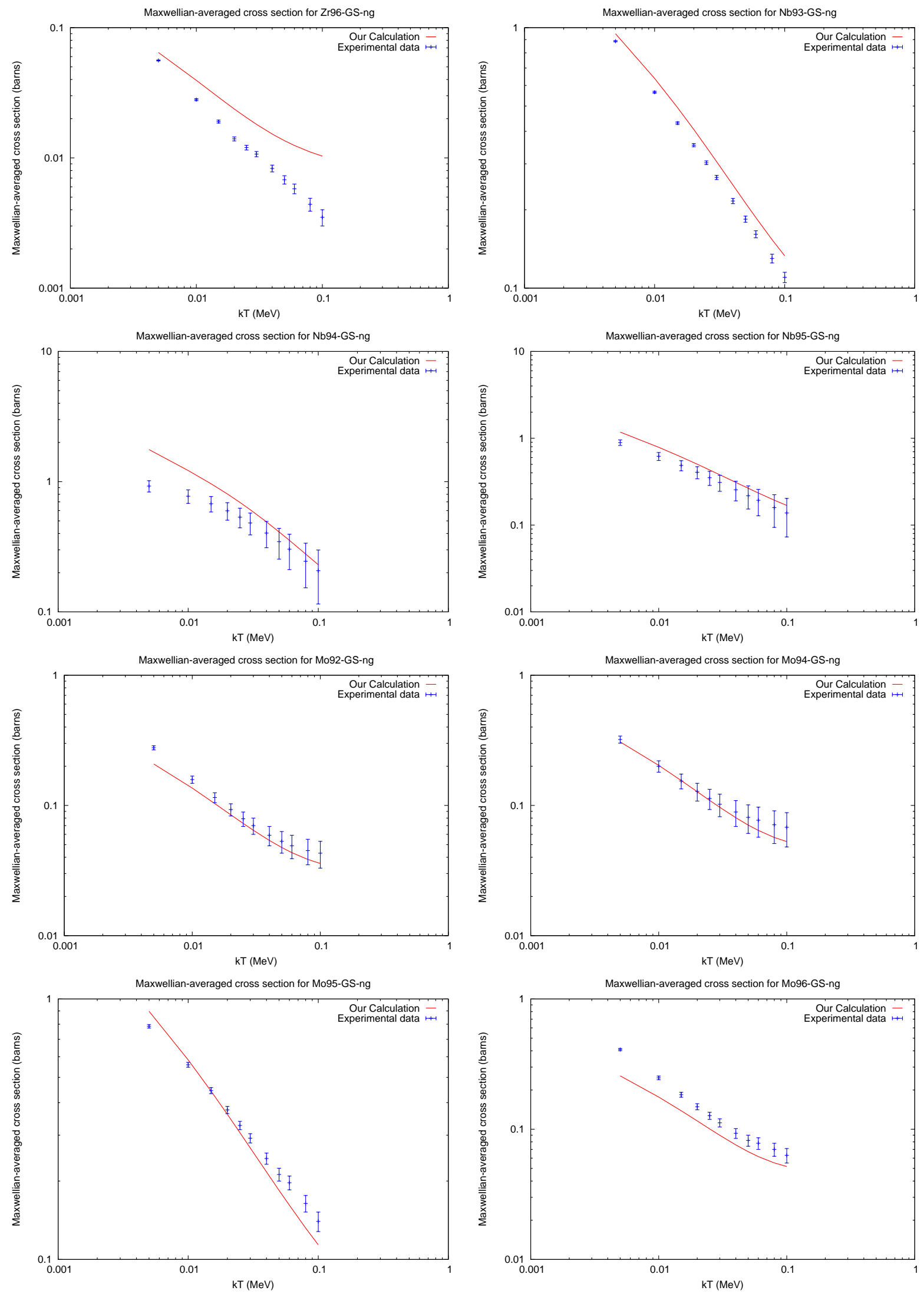

Fig. 26.- (continued) 

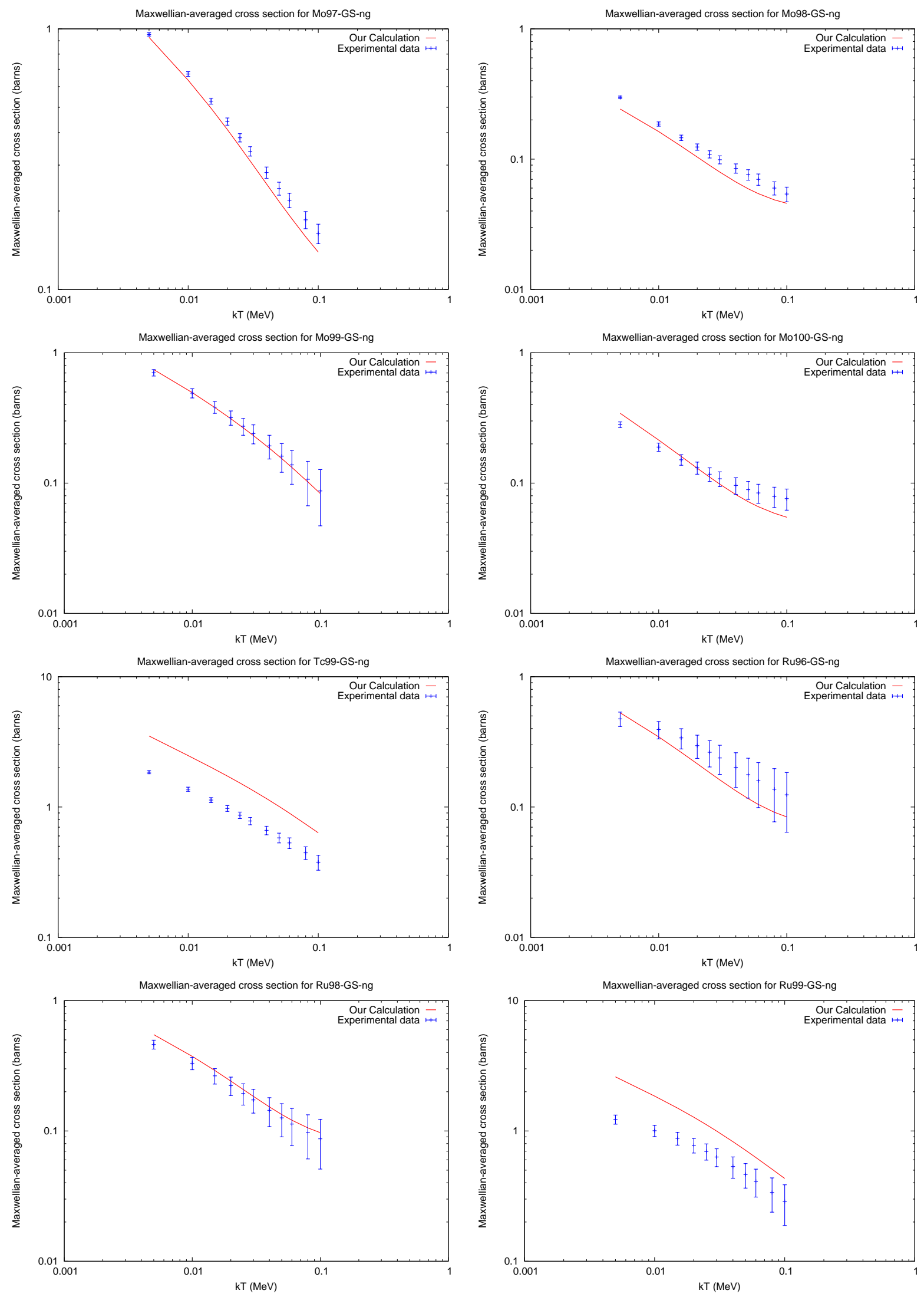

Fig. 26.- (continued) 

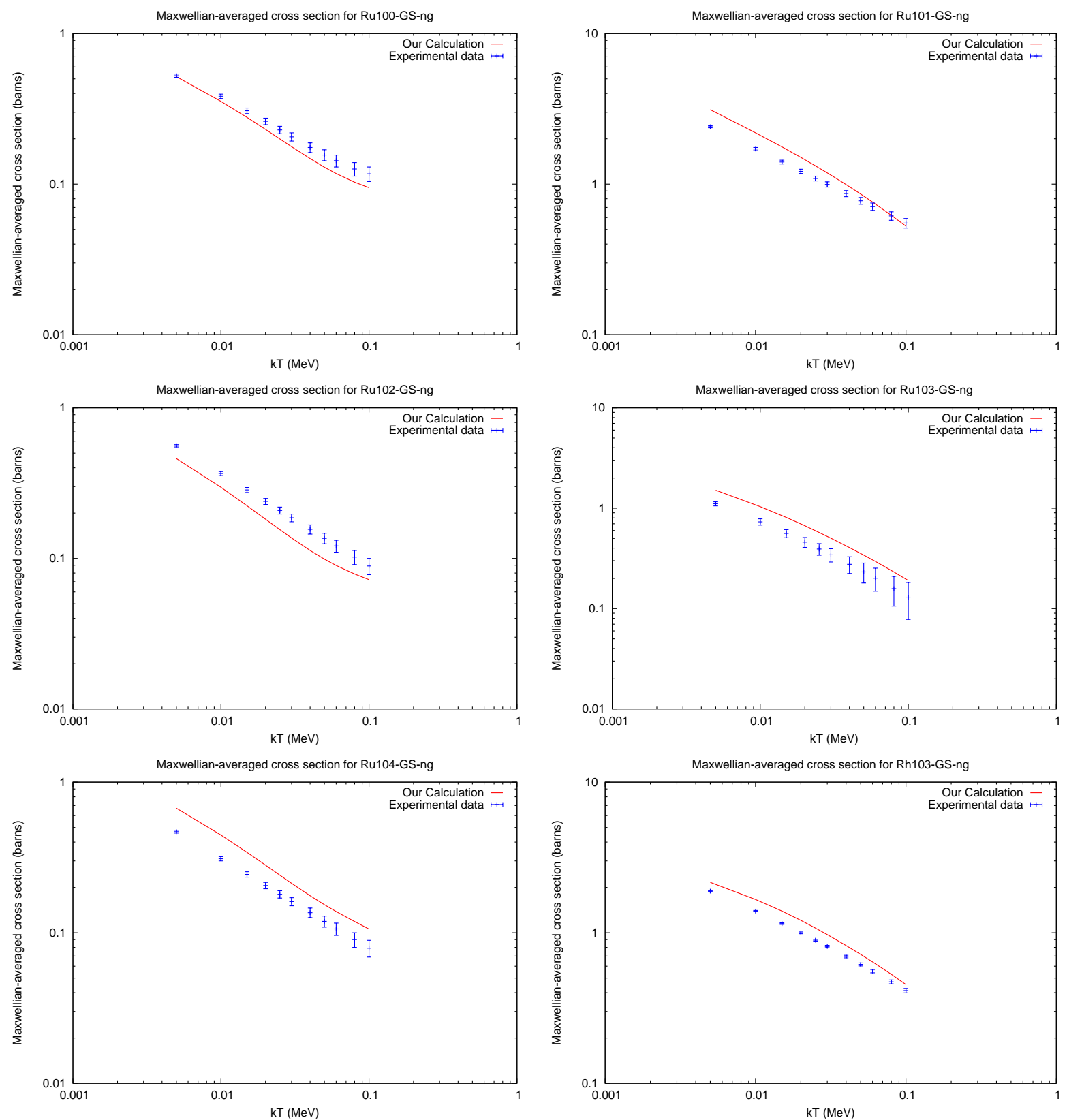

Fig. 26.- (continued) 


\section{C.3. $(\mathrm{n}, 2 \mathrm{n})$}
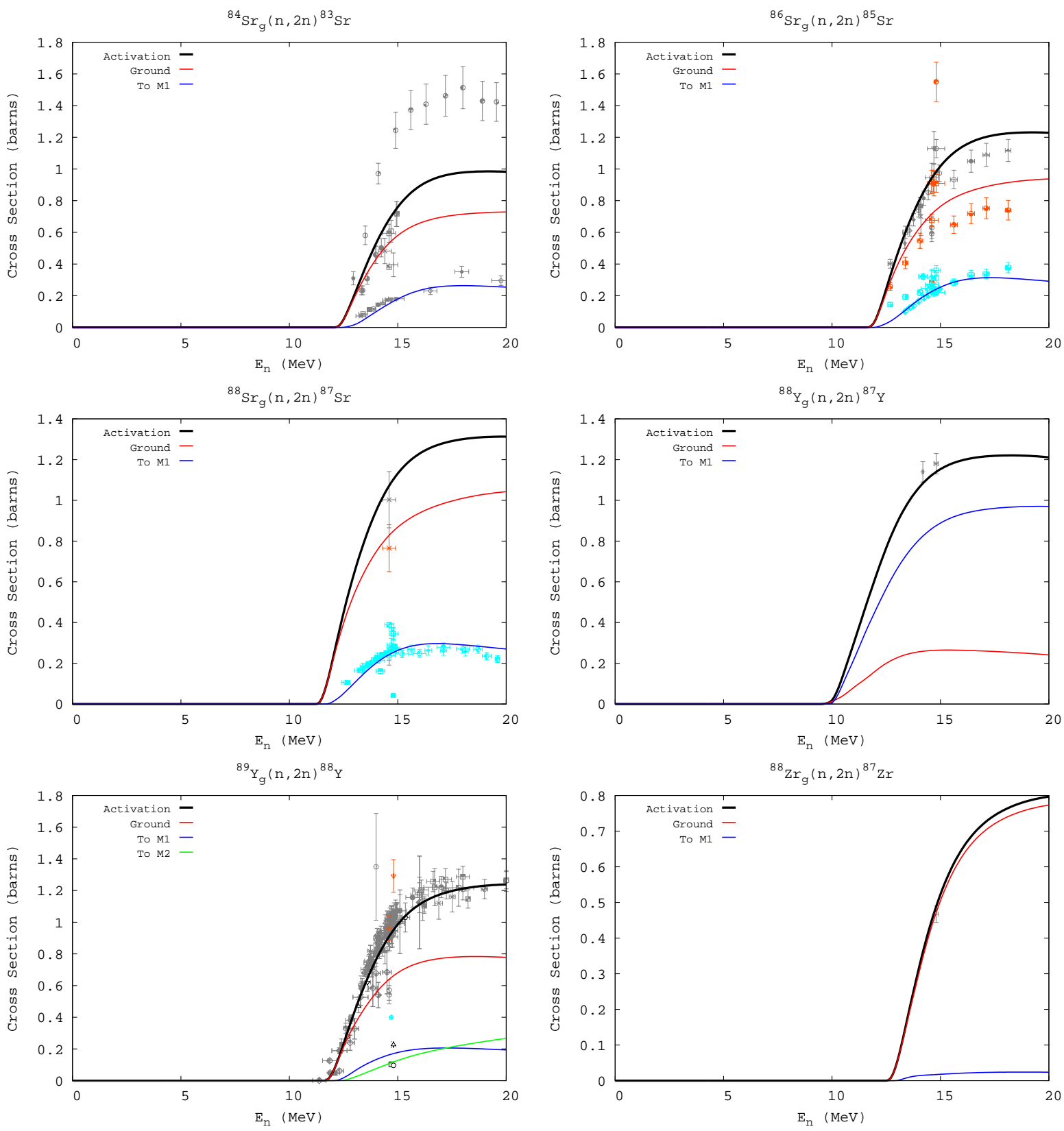

Fig. 27.- Modeled (n,2n) cross sections compared to measurement. The data is taken from (EXFOR 2006). The black, red, and blue solid lines represent our modeled cross sections (total, leading to the ground state, and leading to the first isomer, respectively). The Gery, orange, and light blue data points are measured cross section data (total, ground state, and first isomer). 

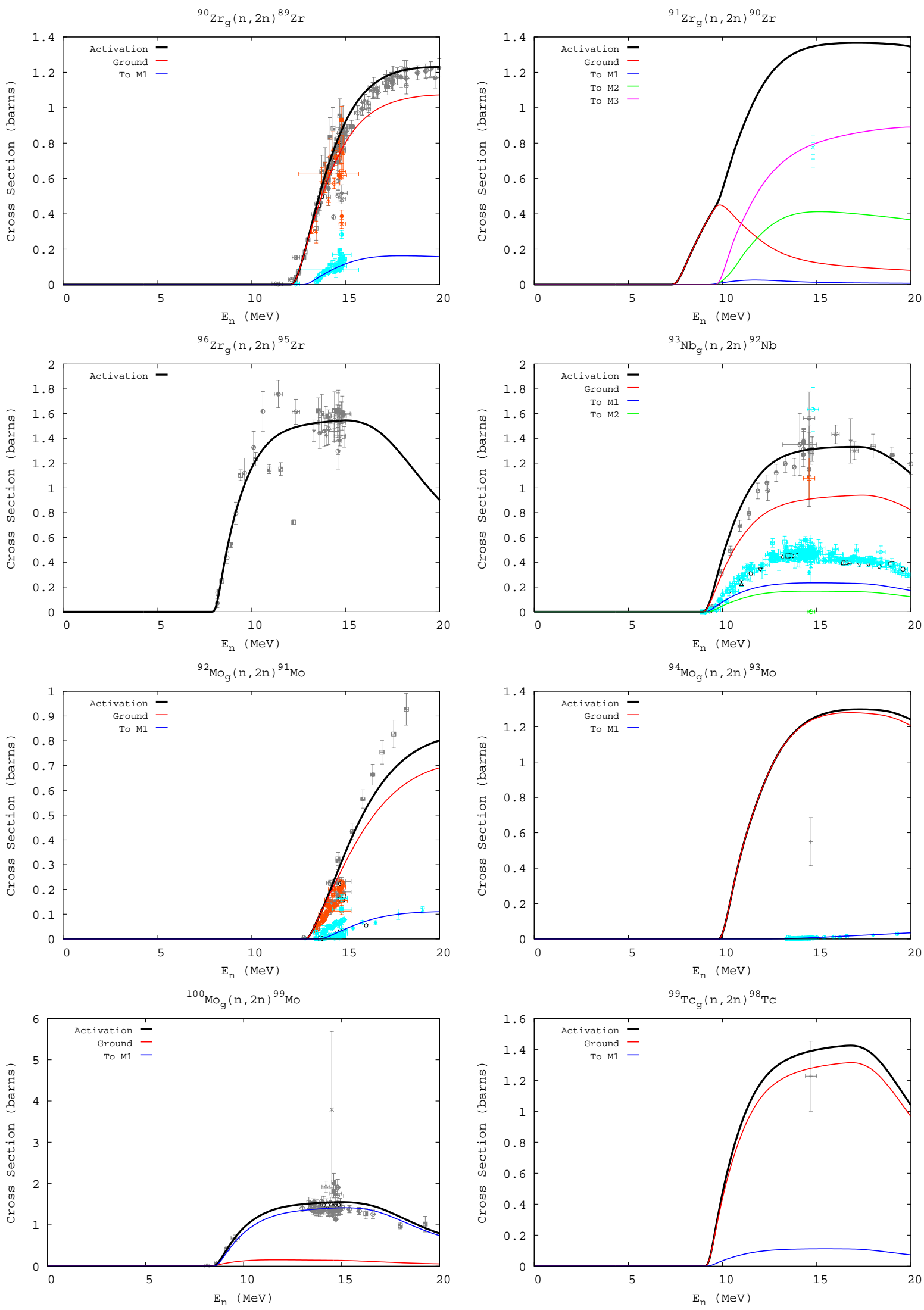

Fig. 27.- (continued) 

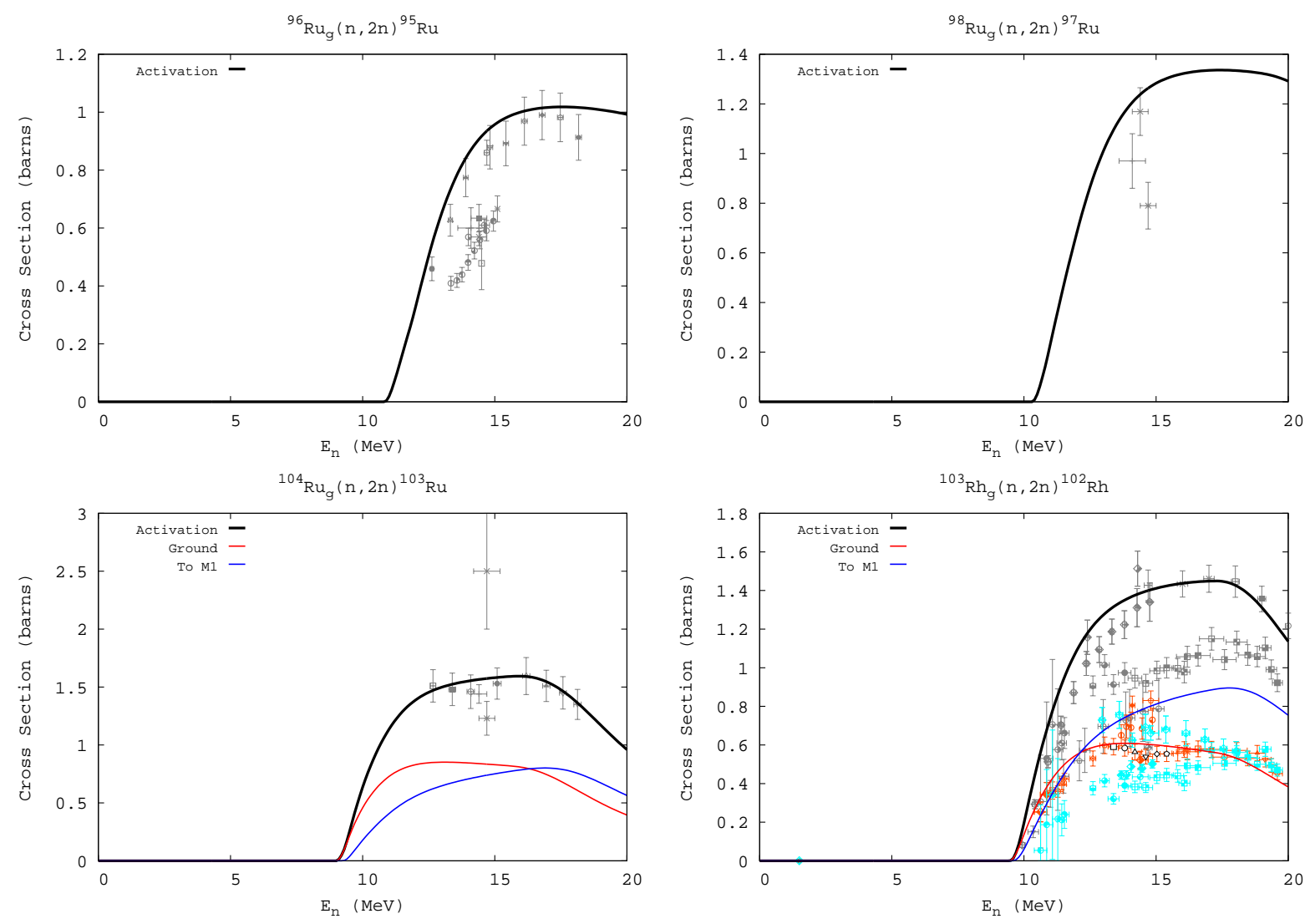

Fig. 27.- (continued) 
C.4. $(\mathrm{n}, 3 \mathrm{n})$
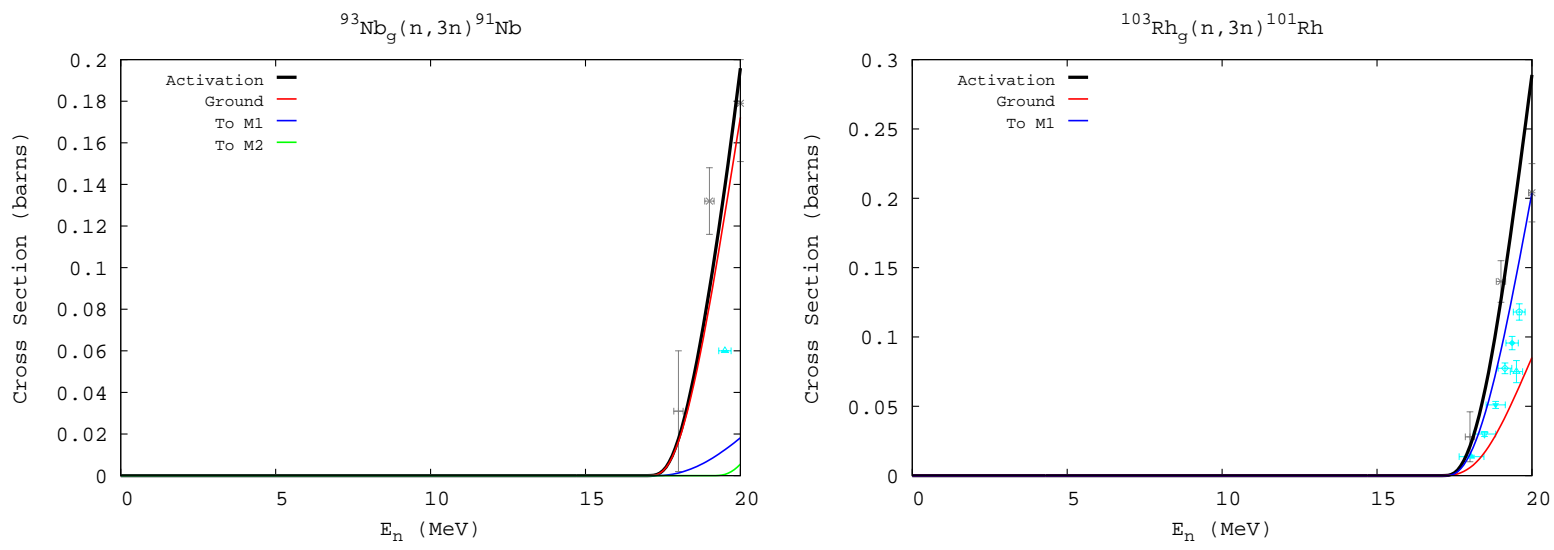

Fig. 28.- Modeled (n,3n) cross sections compared to measurement. The data is taken from (EXFOR 2006). The black, red, and blue solid lines represent our modeled cross sections (total, leading to the ground state, and leading to the first isomer, respectively). The Gery, orange, and light blue data points are measured cross section data (total, ground state, and first isomer). 


\section{C.5. $\left(\mathrm{n}, \mathrm{n}^{\prime}\right)$}
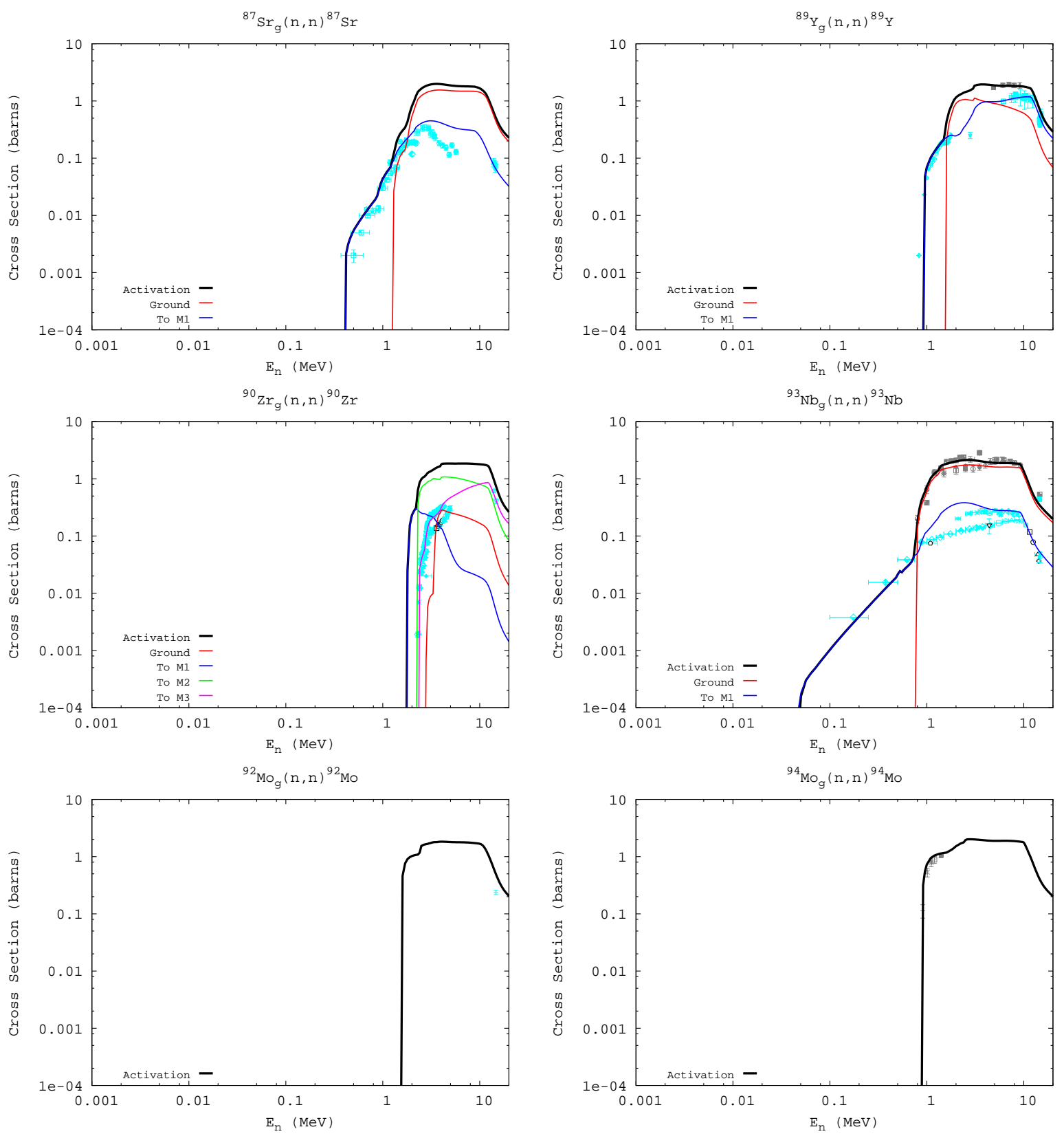

Fig. 29.- Modeled (n,n') cross sections compared to measurement. The data is taken from (EXFOR 2006). The black, red, and blue solid lines represent our modeled cross sections (total, leading to the ground state, and leading to the first isomer, respectively). The Gery, orange, and light blue data points are measured cross section data (total, ground state, and first isomer). 

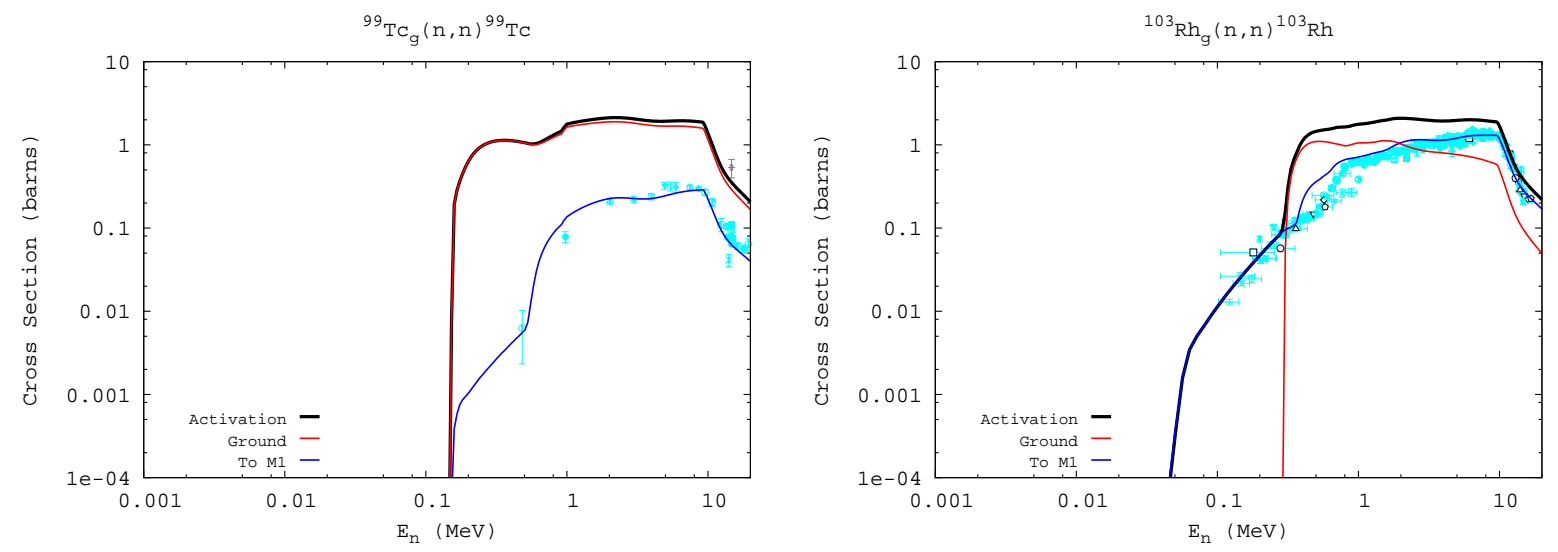

Fig. 29.- (continued) 


\section{C.6. $(n, p)$}
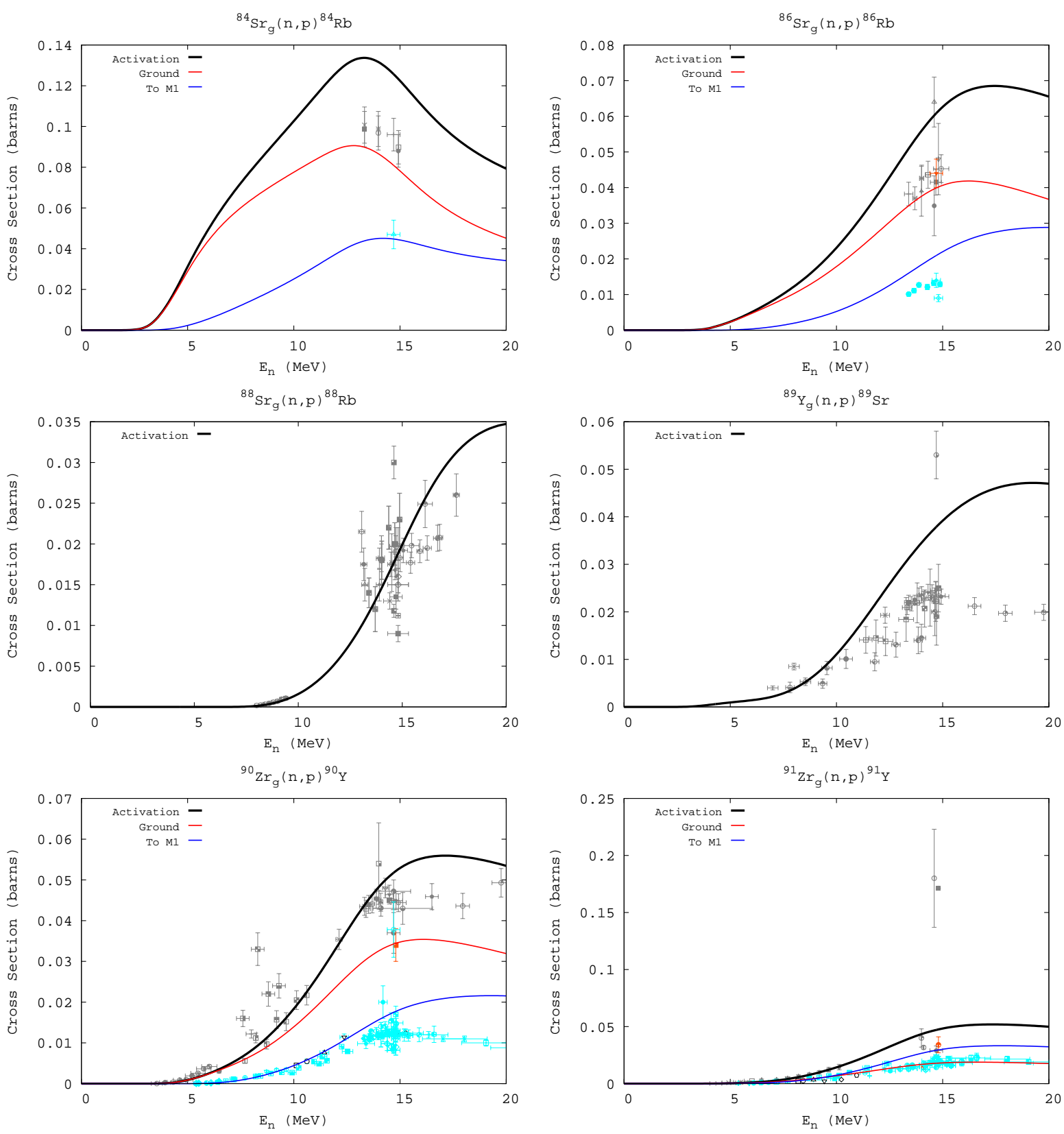

Fig. 30.- Modeled (n,p) cross sections compared to measurement. The data is taken from (EXFOR 2006). The black, red, and blue solid lines represent our modeled cross sections (total, leading to the ground state, and leading to the first isomer, respectively). The Gery, orange, and light blue data points are measured cross section data (total, ground state, and first isomer). 

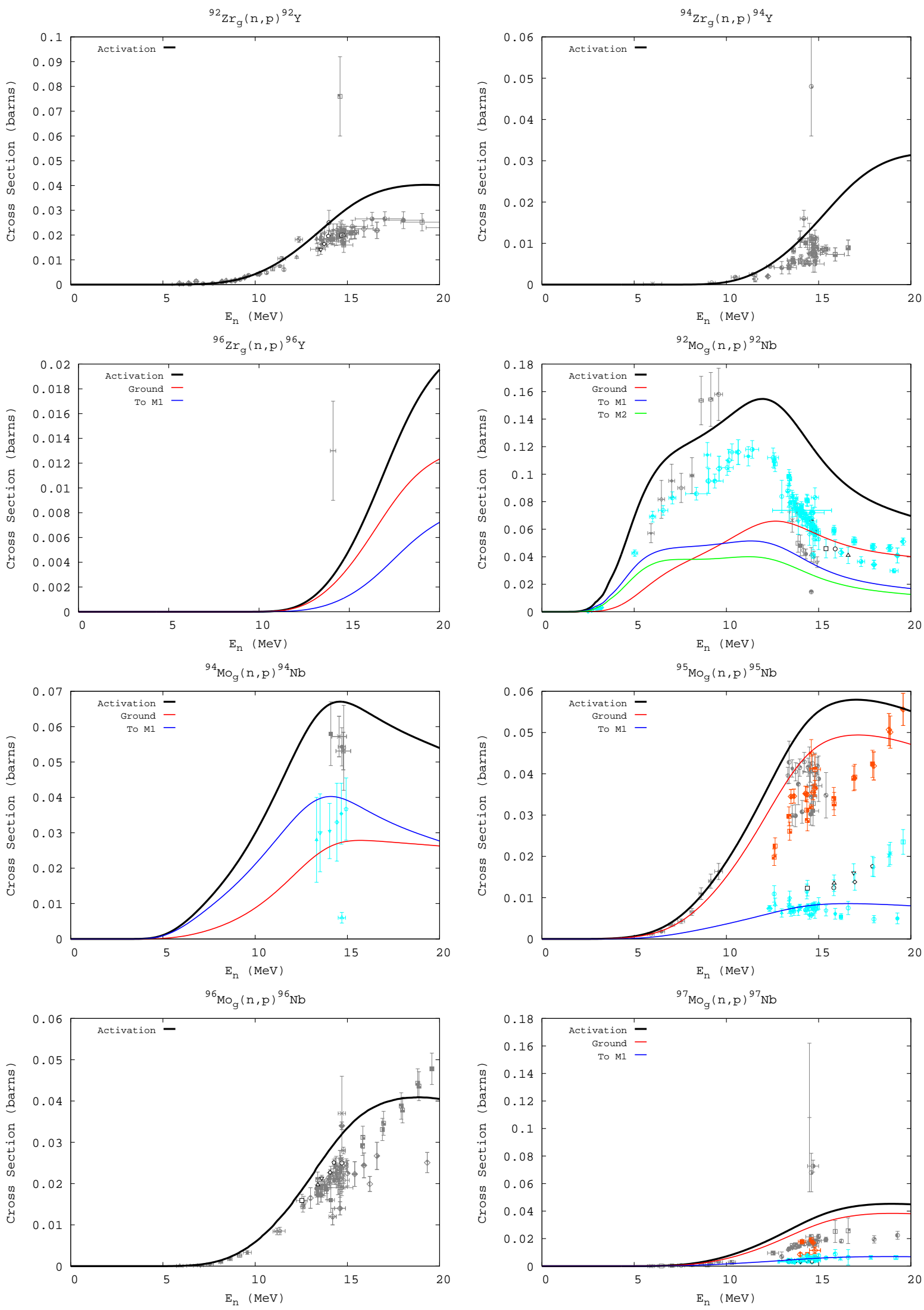

Fig. 30.- (continued) 

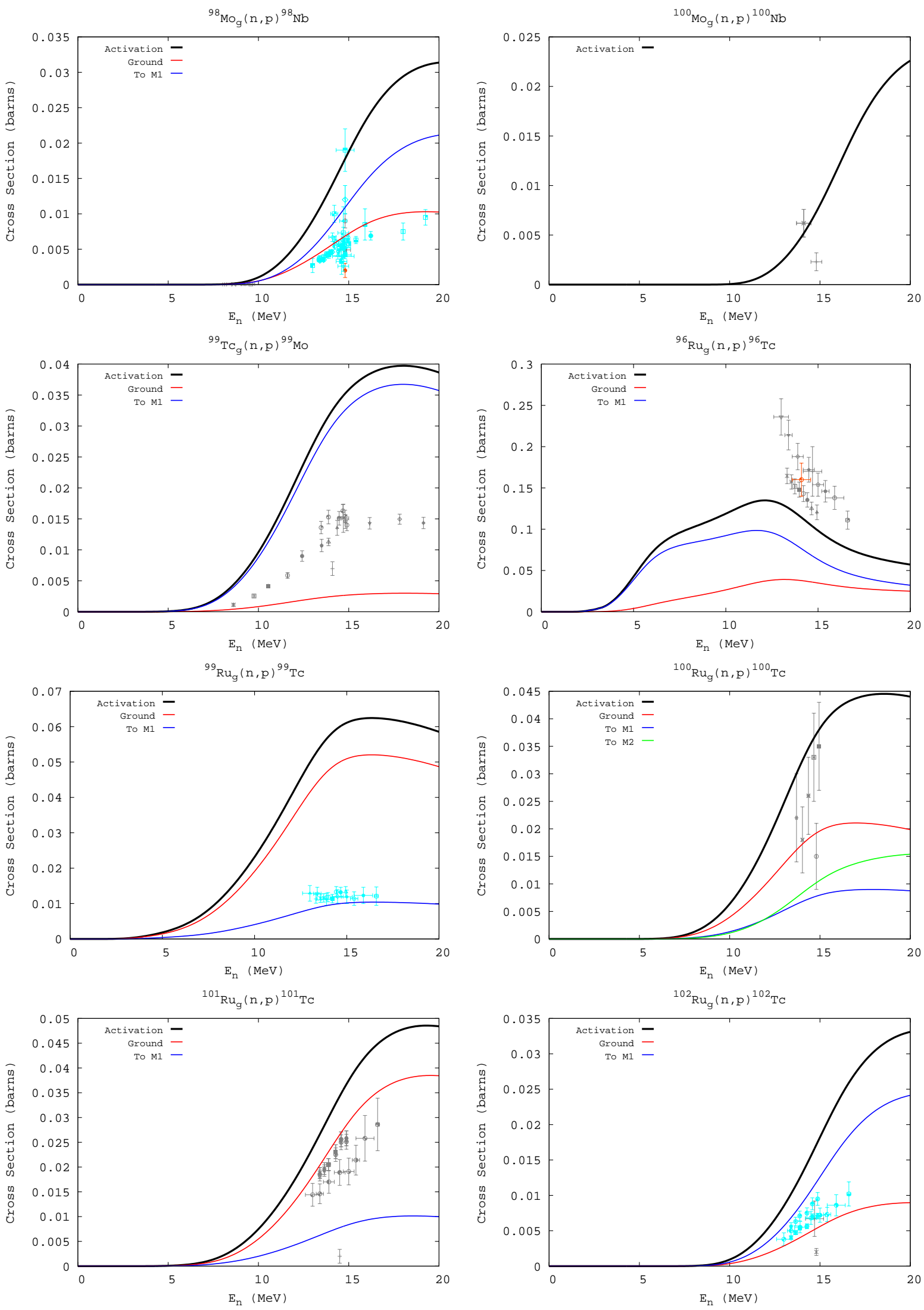

Fig. 30.- (continued) 

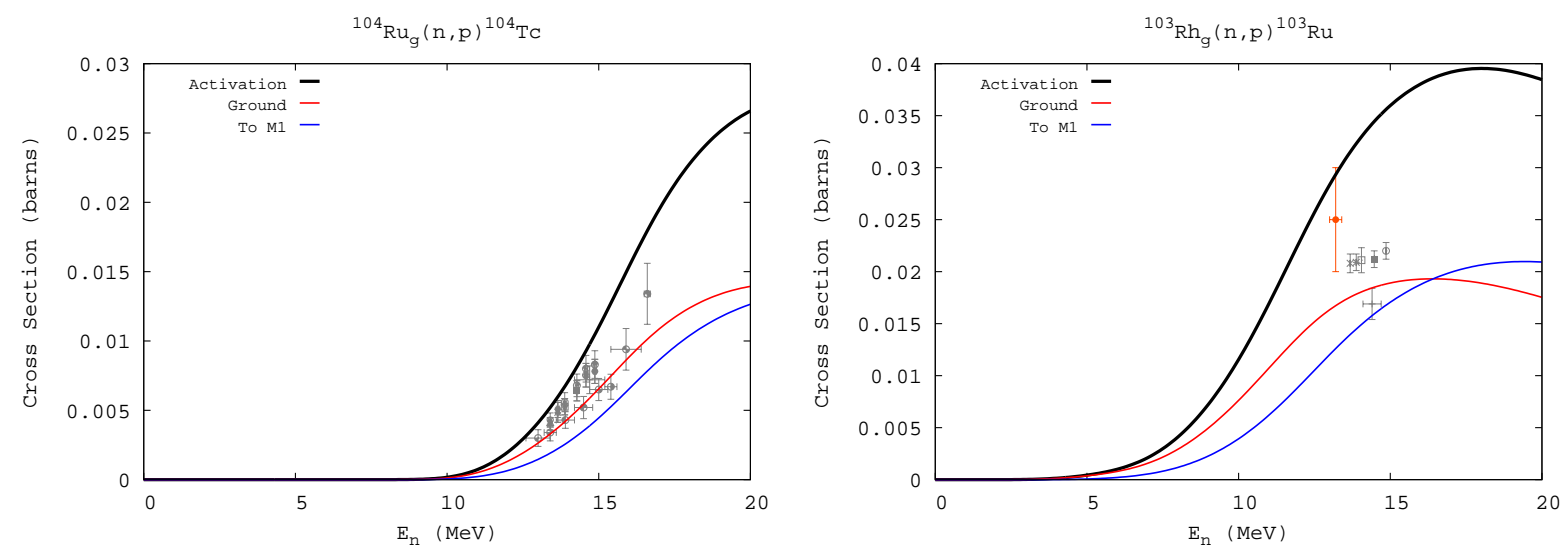

Fig. 30.- (continued) 


\section{C.7. $(\mathbf{n}, \mathrm{np})$}
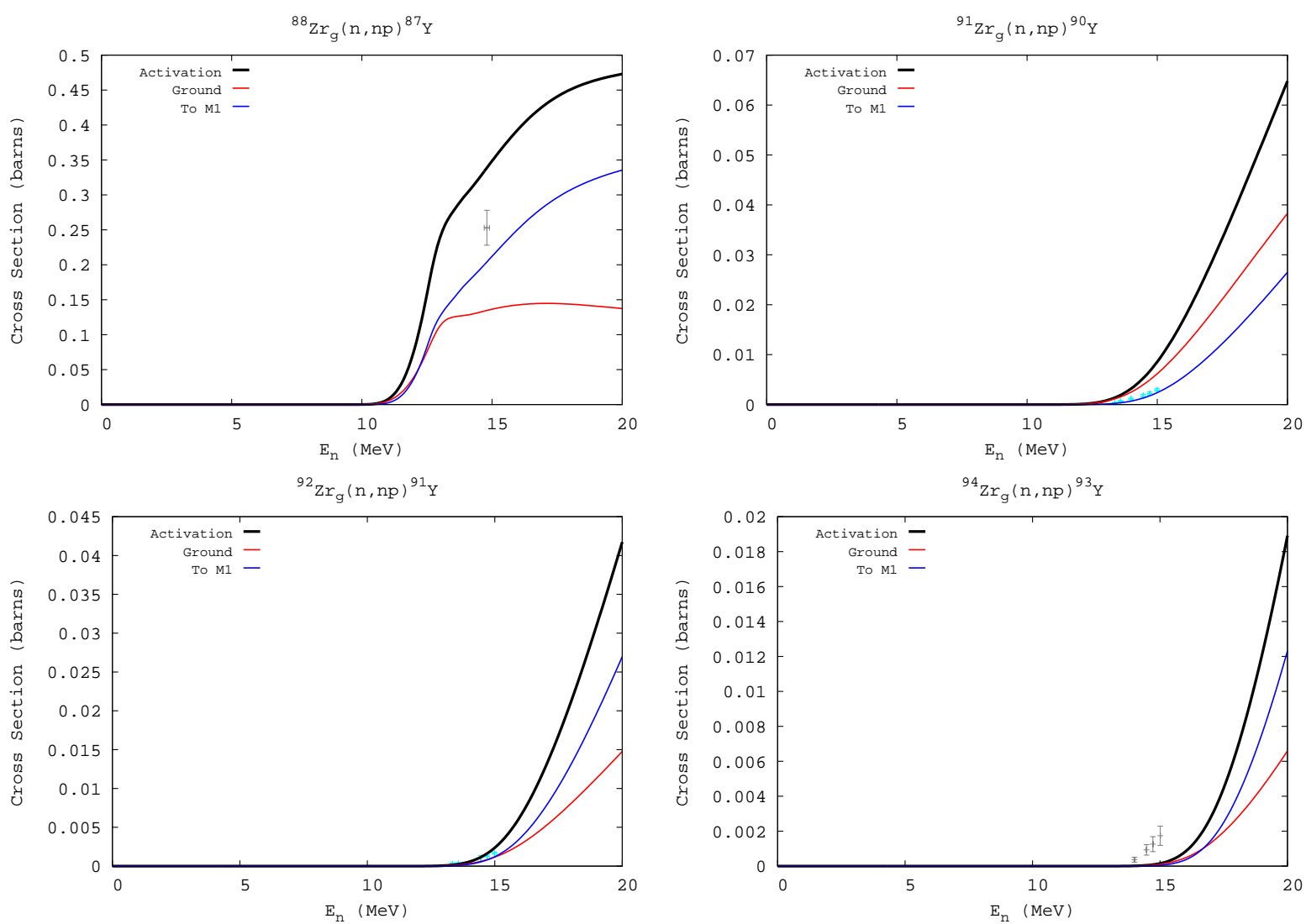

${ }^{96} \mathrm{Zr}_{\mathrm{g}}(\mathrm{n}, \mathrm{np}){ }^{95} \mathrm{Y}$
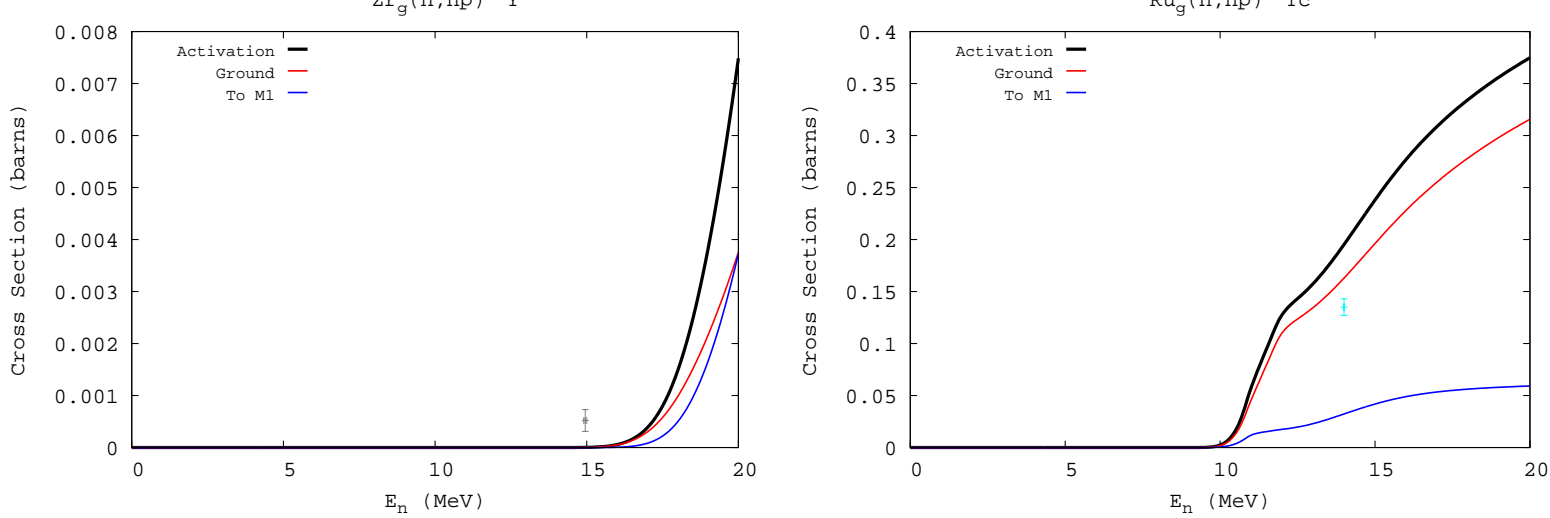

Fig. 31.- Modeled (n,np) cross sections compared to measurement. The data is taken from (EXFOR 2006). The black, red, and blue solid lines represent our modeled cross sections (total, leading to the ground state, and leading to the first isomer, respectively). The Gery, orange, and light blue data points are measured cross section data (total, ground state, and first isomer). 

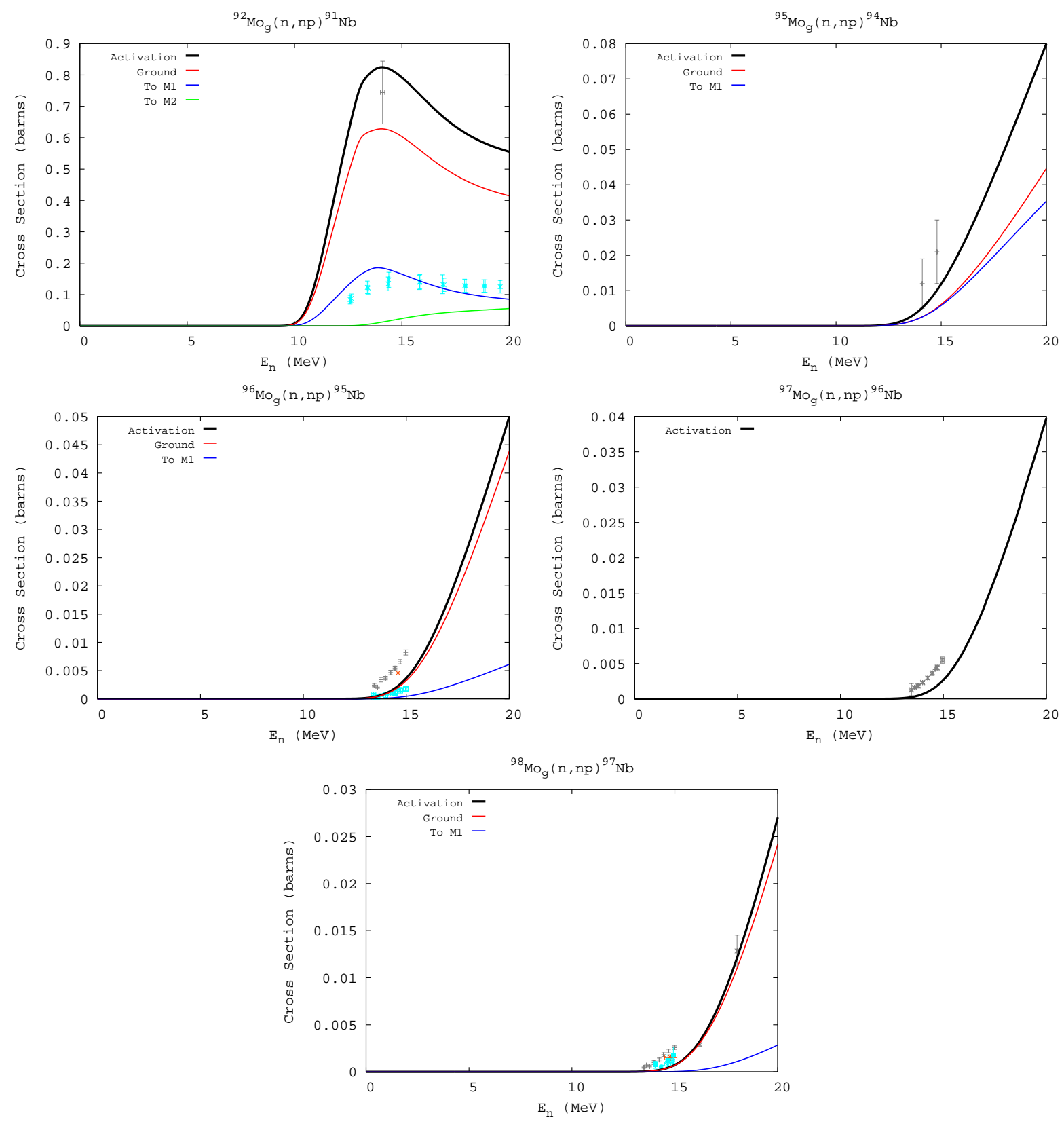

Fig. 31.- (continued) 


\section{C.8. $(\mathrm{n}, \alpha)$}
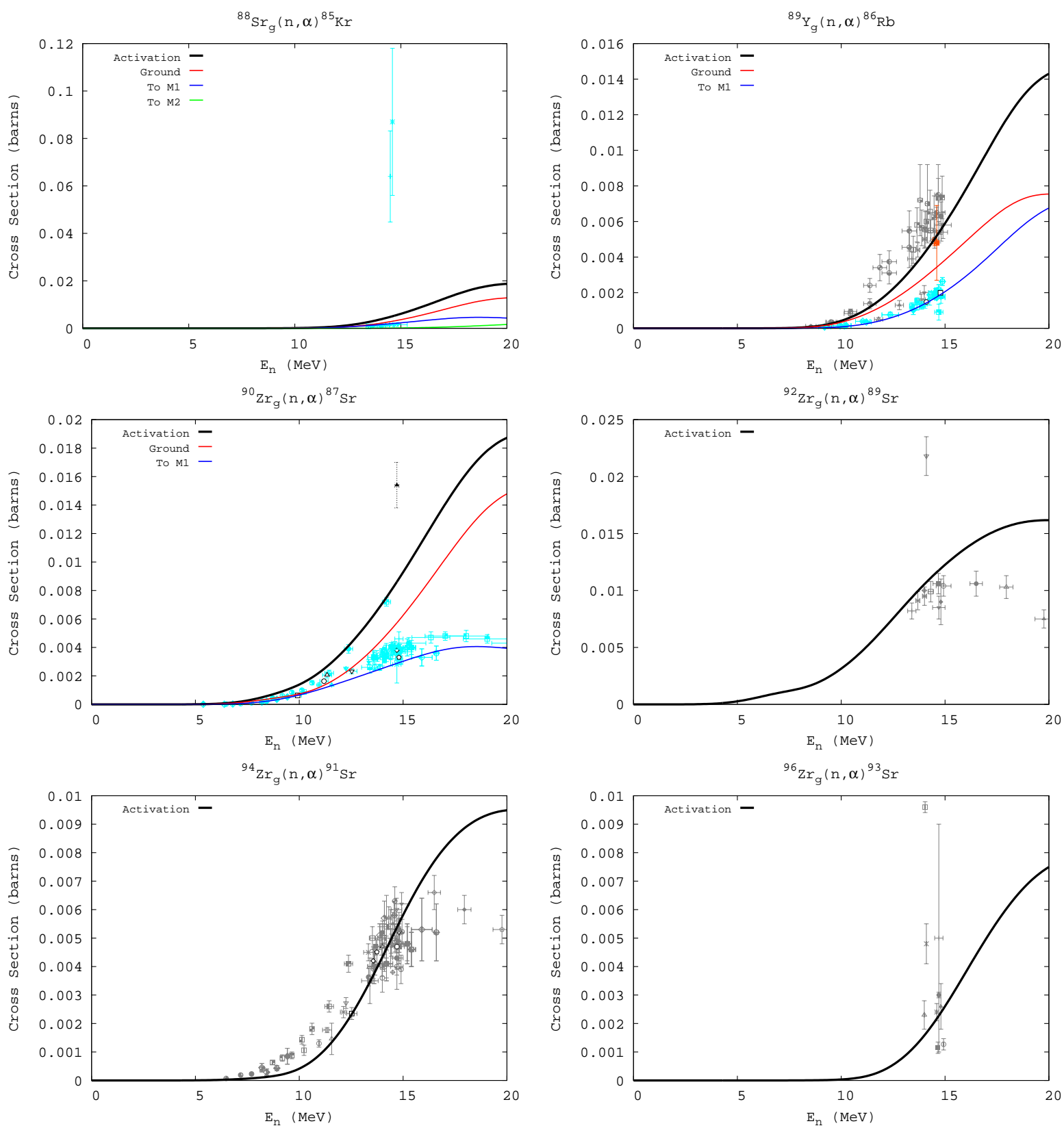

Fig. 32.- Modeled (n, $\alpha$ ) cross sections compared to measurement. The data is taken from (EXFOR 2006). The black, red, and blue solid lines represent our modeled cross sections (total, leading to the ground state, and leading to the first isomer, respectively). The Gery, orange, and light blue data points are measured cross section data (total, ground state, and first isomer). 

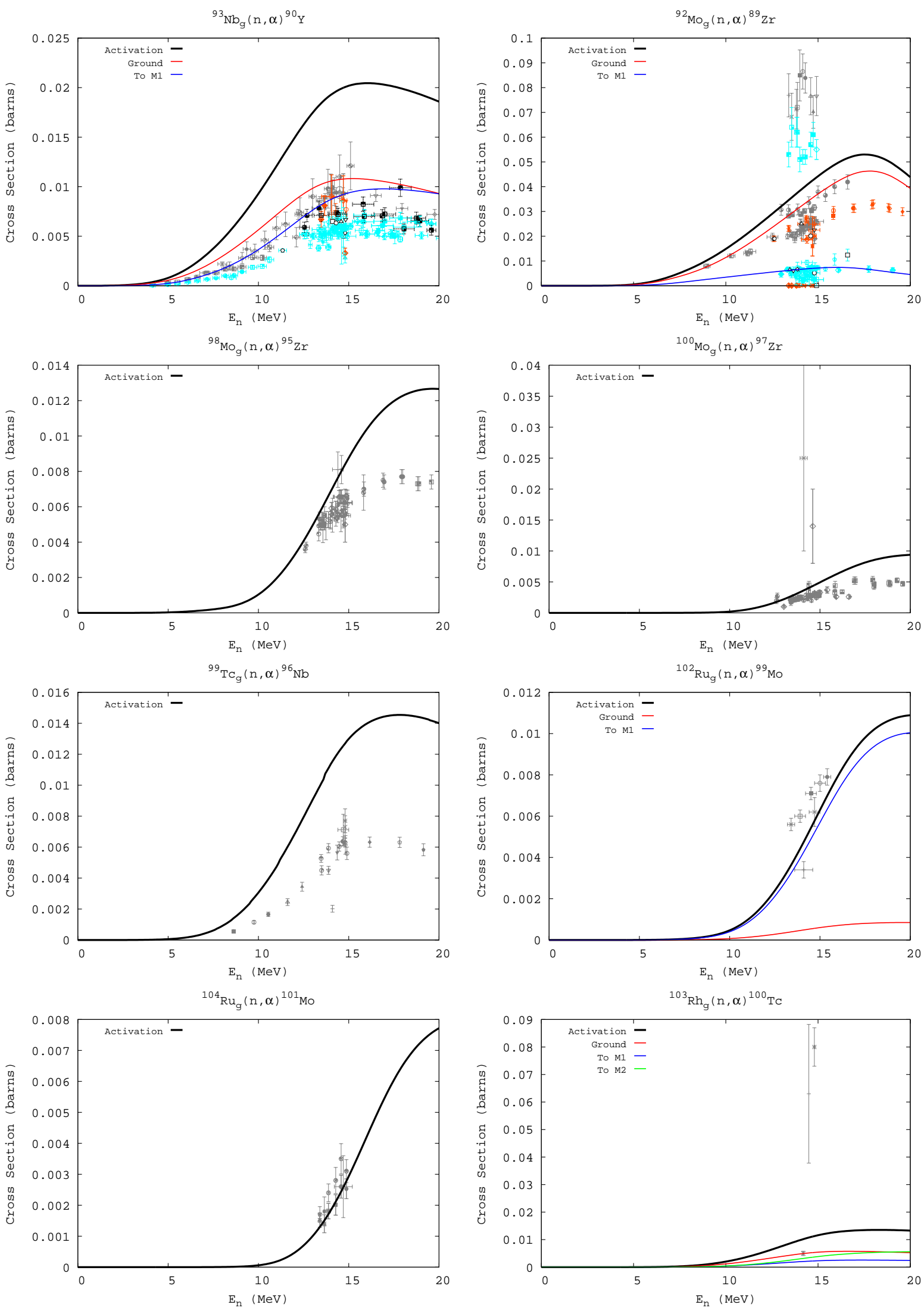

Fig. 32.- (continued) 


\section{C.9. $(\mathbf{n}, \mathbf{n} \alpha)$}
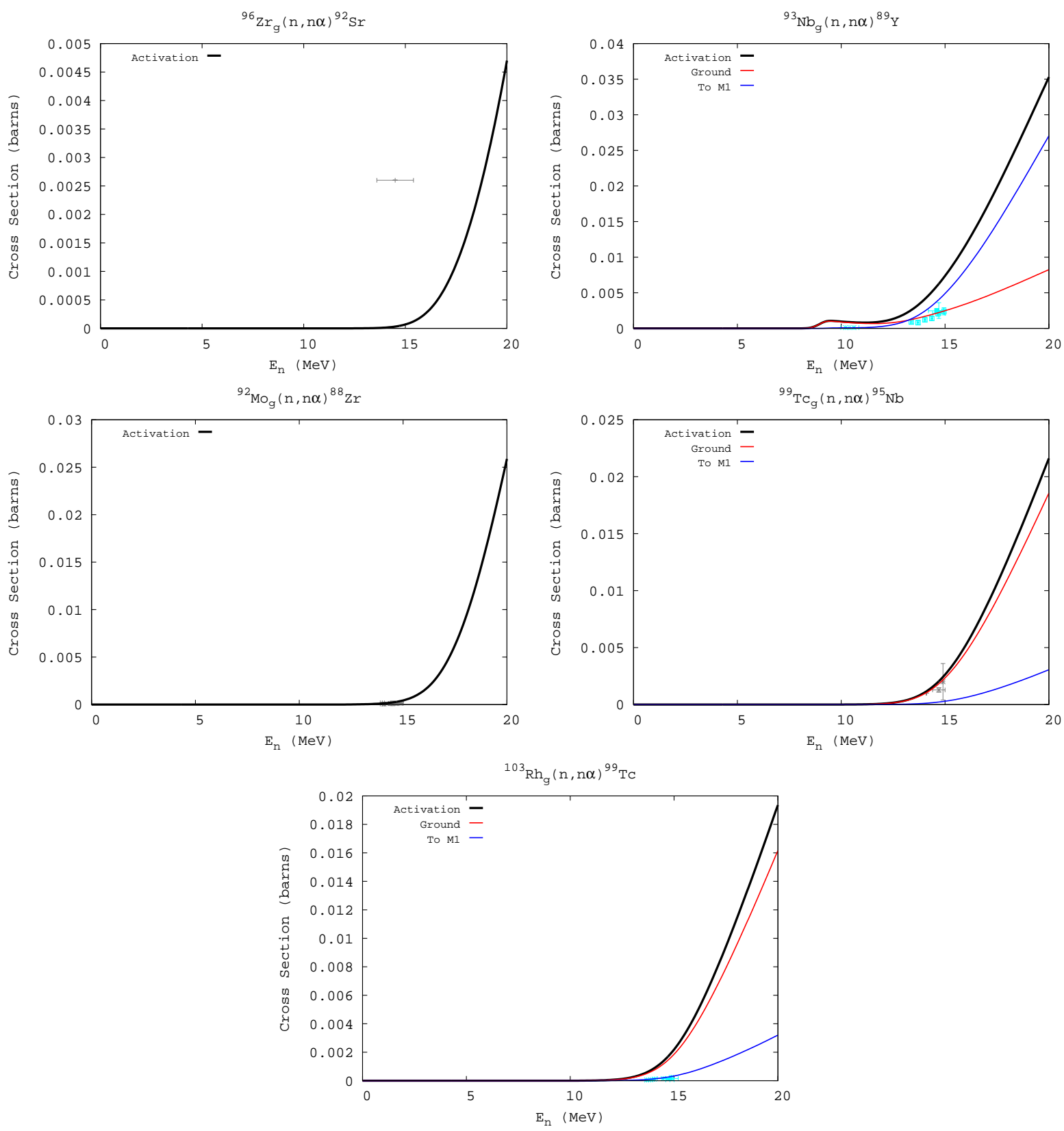

Fig. 33.- Modeled (n,n $\alpha)$ cross sections compared to measurement. The data is taken from (EXFOR 2006). The black, red, and blue solid lines represent our modeled cross sections (total, leading to the ground state, and leading to the first isomer, respectively). The Gery, orange, and light blue data points are measured cross section data (total, ground state, and first isomer). 
C.10. $(n, d)$
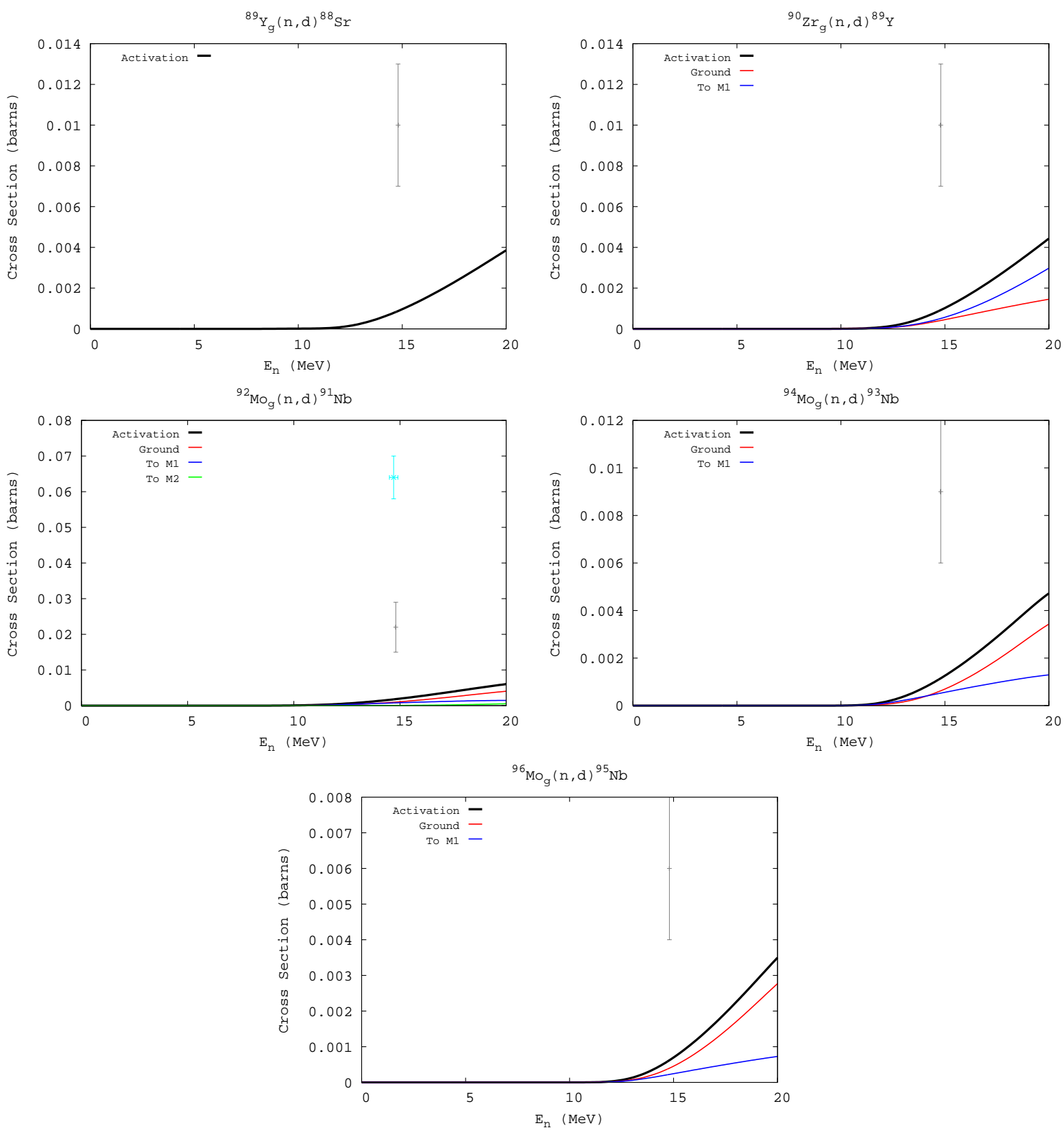

Fig. 34.- Modeled (n,d) cross sections compared to measurement. The data is taken from (EXFOR 2006). The black, red, and blue solid lines represent our modeled cross sections (total, leading to the ground state, and leading to the first isomer, respectively). The Gery, orange, and light blue data points are measured cross section data (total, ground state, and first isomer). 
C.11. (p,n)
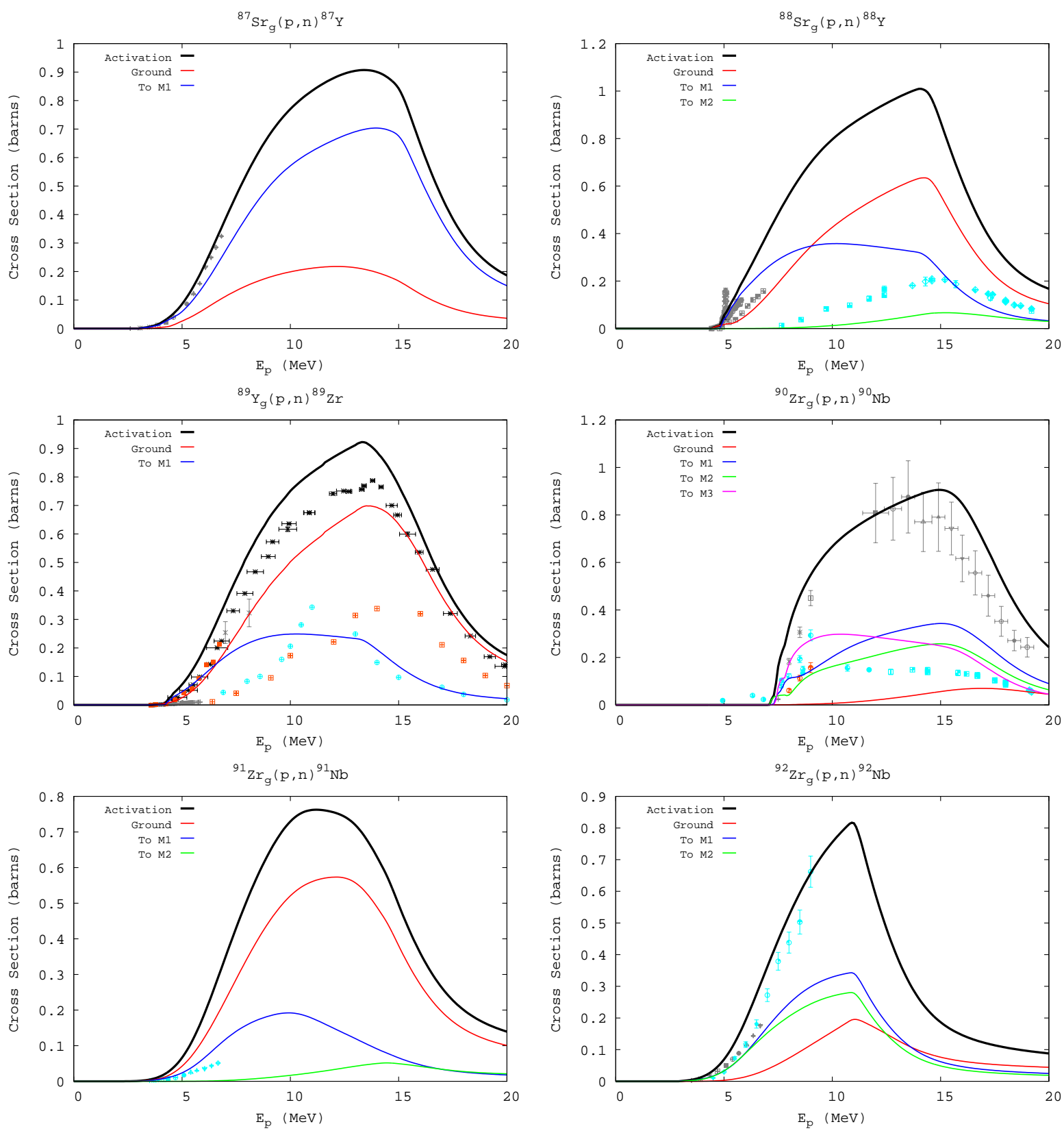

Fig. 35.- Modeled (p,n) cross sections compared to measurement. The data is taken from (EXFOR 2006). The black, red, and blue solid lines represent our modeled cross sections (total, leading to the ground state, and leading to the first isomer, respectively). The Gray, orange, and light blue data points are measured cross section data (total, ground state, and first isomer). 

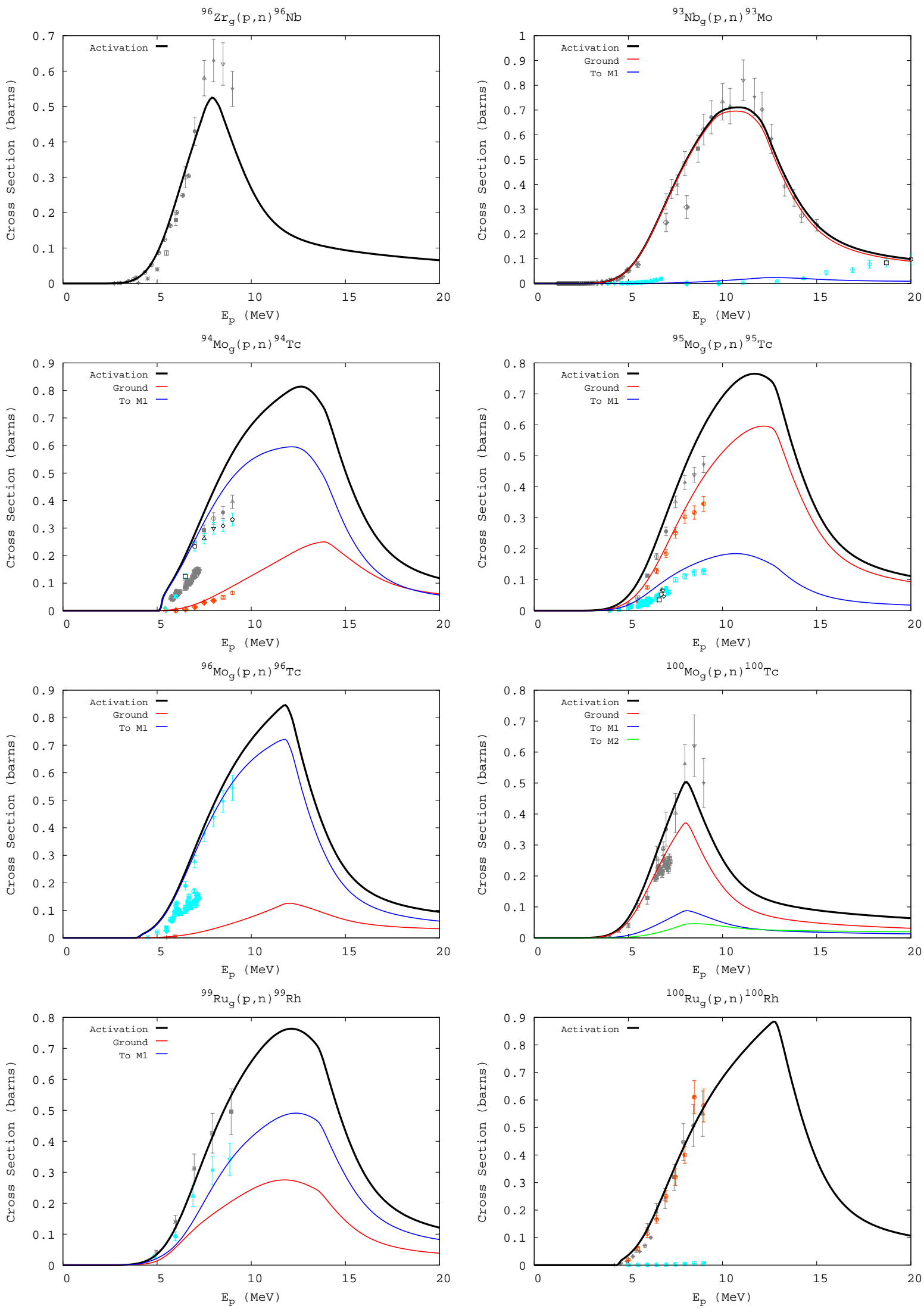

Fig. 35.- (continued) 

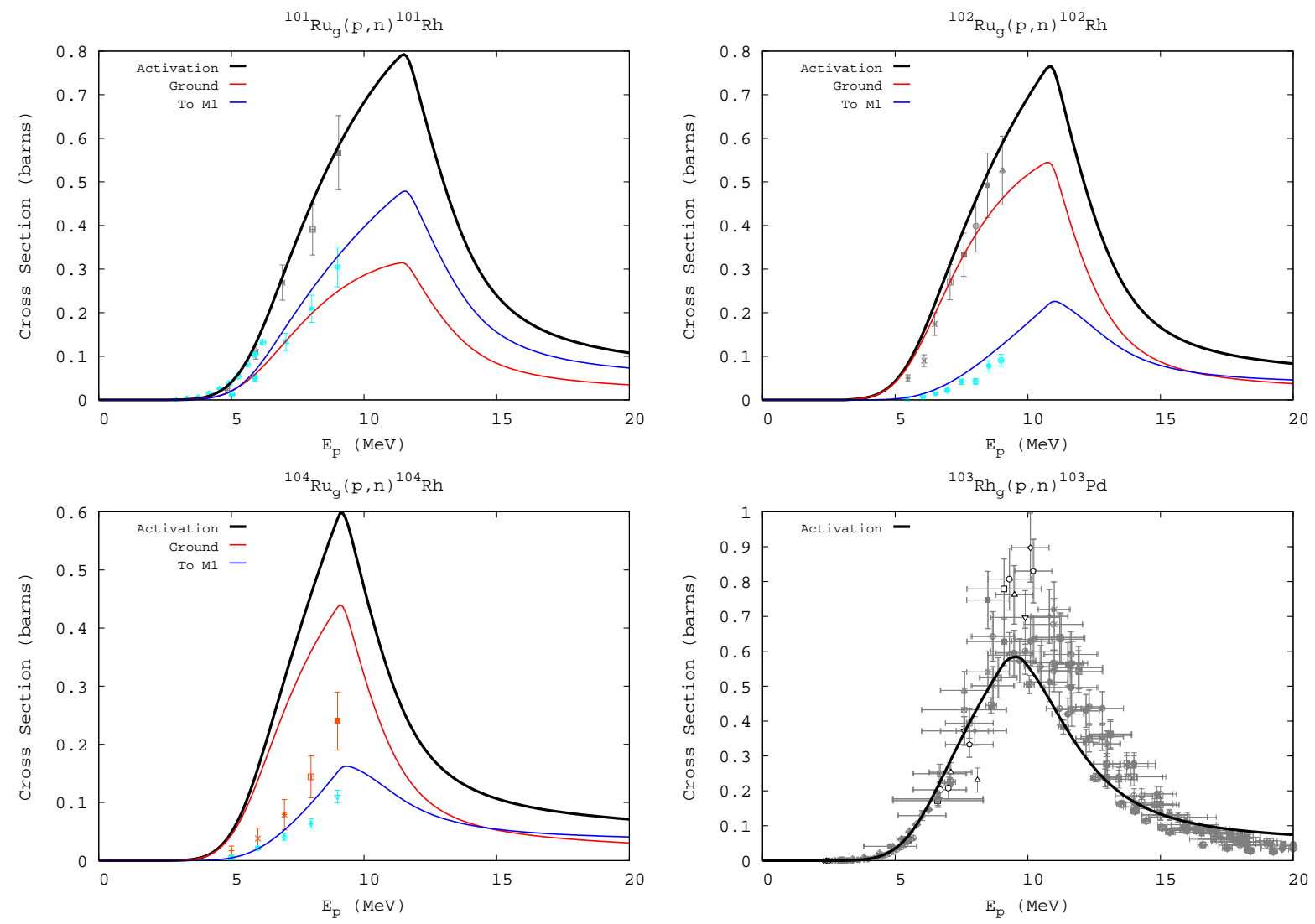

Fig. 35.- (continued) 


\section{C.12. $(p, 2 n)$}
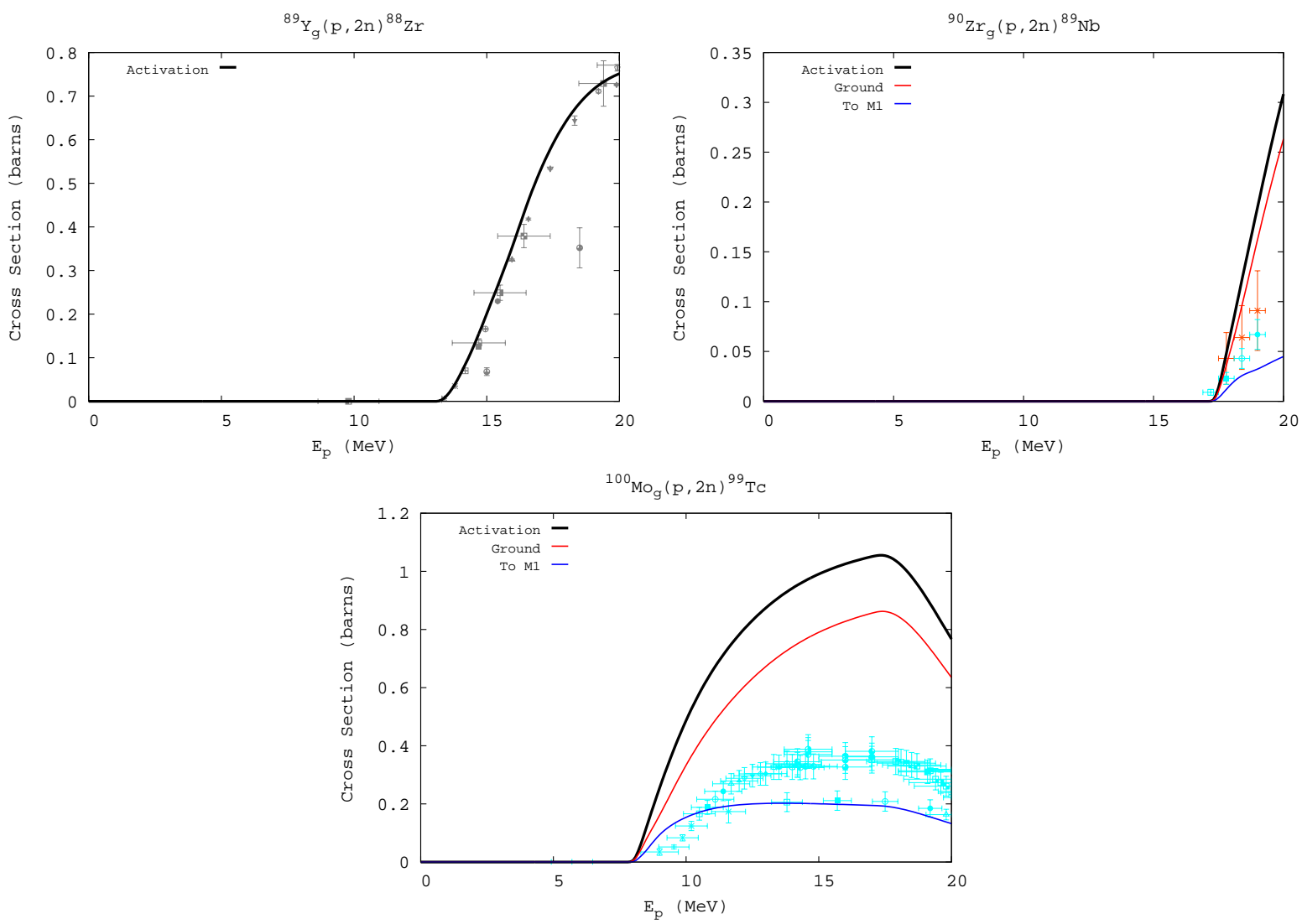

Fig. 36.- Modeled (p,2n) cross sections compared to measurement. The data is taken from (EXFOR 2006). The black, red, and blue solid lines represent our modeled cross sections (total, leading to the ground state, and leading to the first isomer, respectively). The Rey, orange, and light blue data points are measured cross section data (total, ground state, and first isomer). 
C.13. (p,np)
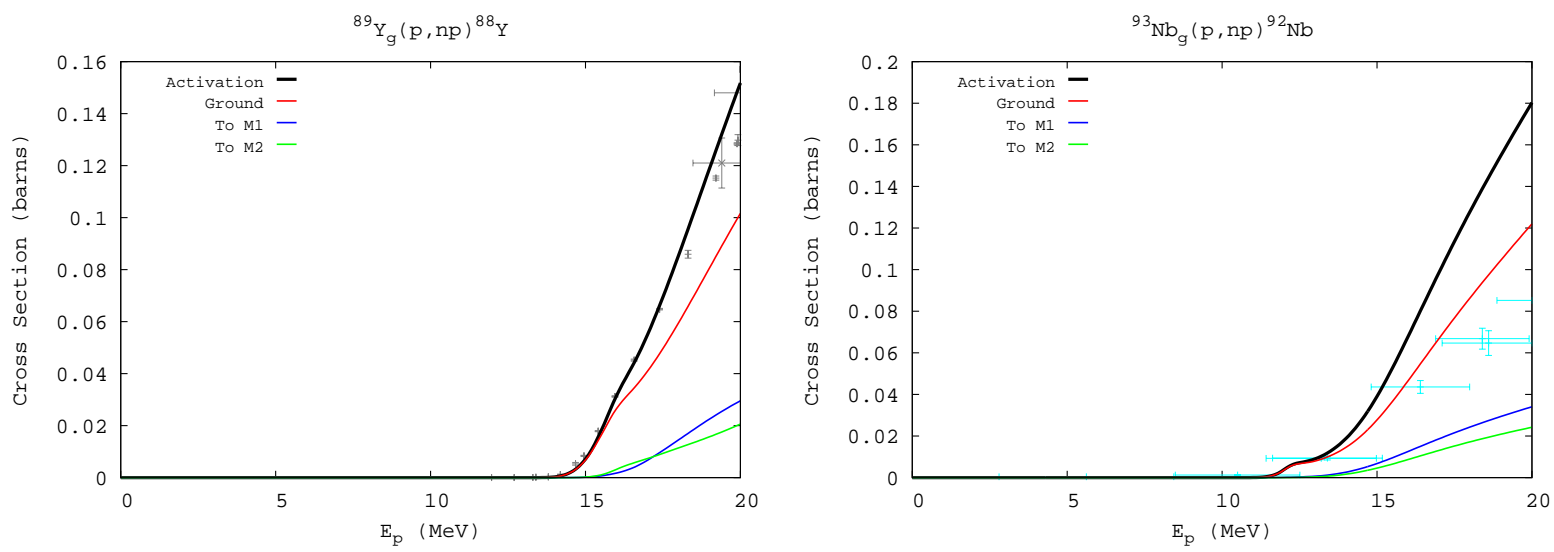

Fig. 37.- Modeled (p,np) cross sections compared to measurement. The data is taken from (EXFOR 2006). The black, red, and blue solid lines represent our modeled cross sections (total, leading to the ground state, and leading to the first isomer, respectively). The Frey, orange, and light blue data points are measured cross section data (total, ground state, and first isomer). 


\section{C.14. (p,p')}

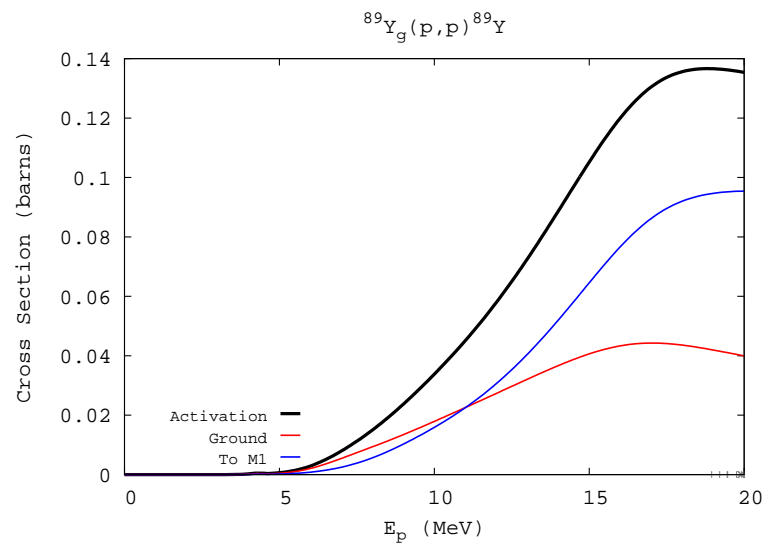

Fig. 38.- Modeled (p,p') cross sections compared to measurement. The data is taken from (EXFOR 2006). The black, red, and blue solid lines represent our modeled cross sections (total, leading to the ground state, and leading to the first isomer, respectively). The Gorey, orange, and light blue data points are measured cross section data (total, ground state, and first isomer). 
C.15. $(p, \gamma)$
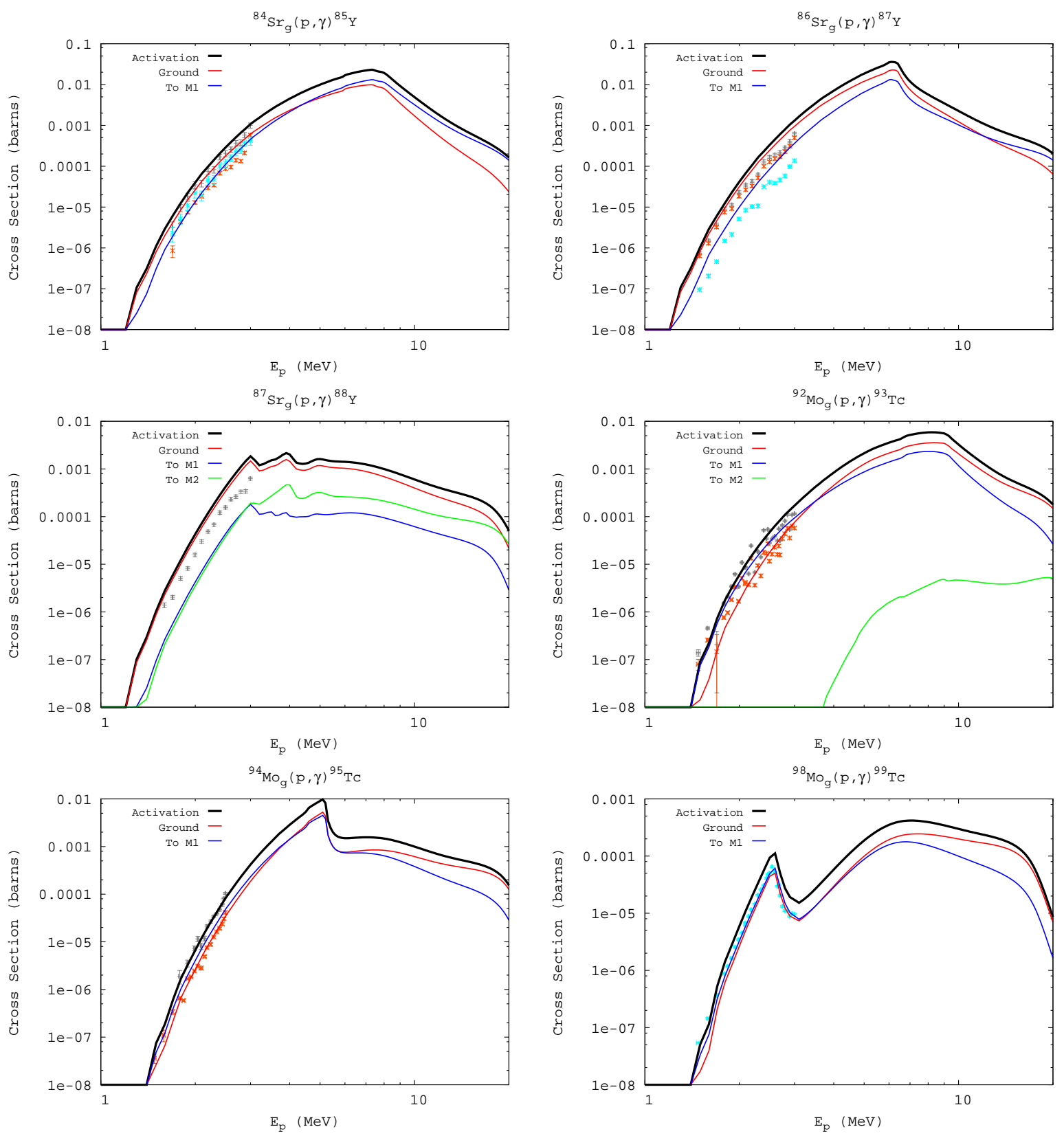

Fig. 39.- Modeled (p, $\gamma$ ) cross sections compared to measurement. The data is taken from (EXFOR 2006). The black, red, and blue solid lines represent our modeled cross sections (total, leading to the ground state, and leading to the first isomer, respectively). The Garey, orange, and light blue data points are measured cross section data (total, ground state, and first isomer). 

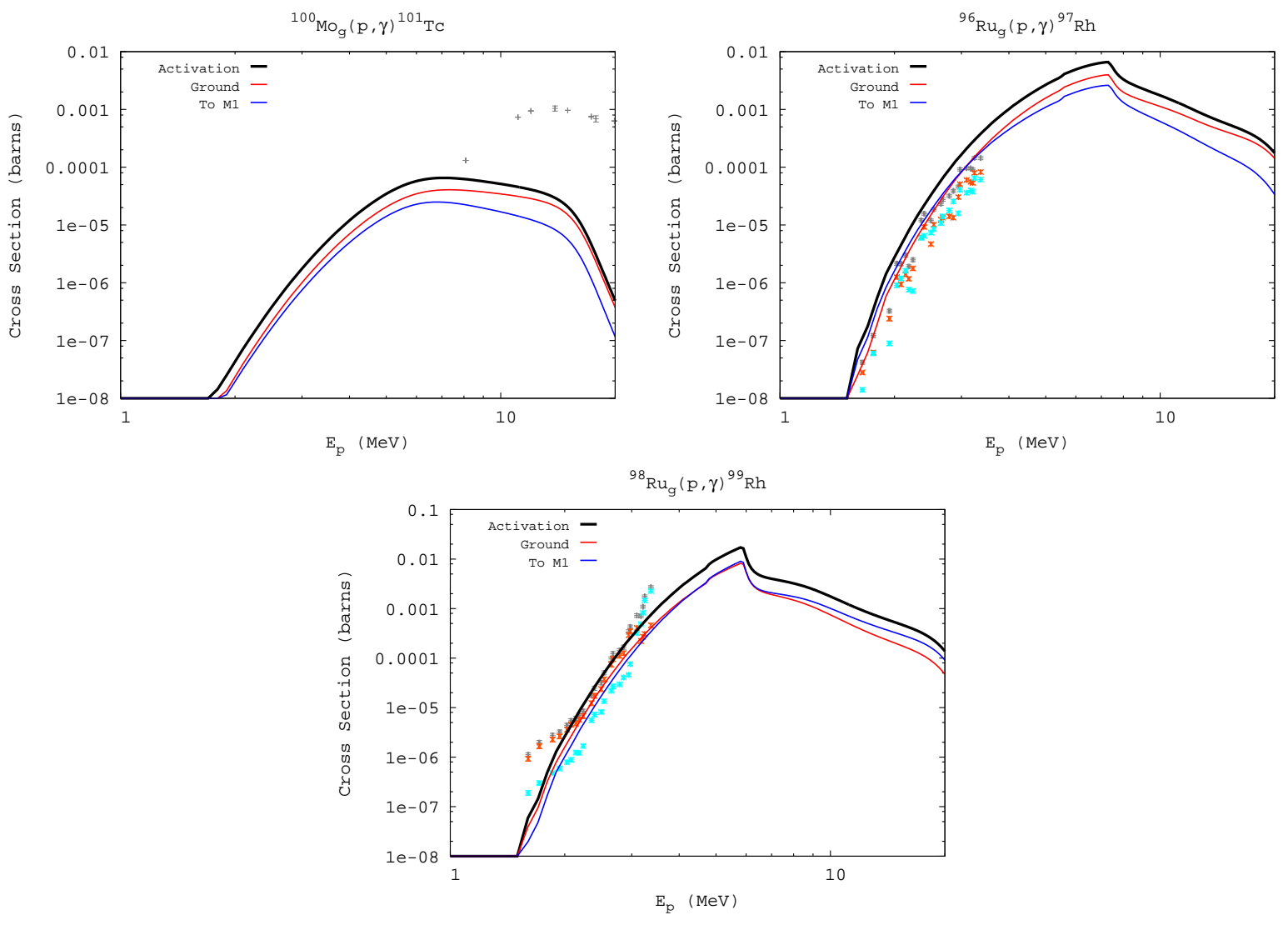

Fig. 39.- (continued) 
C.16. $(d, n)$
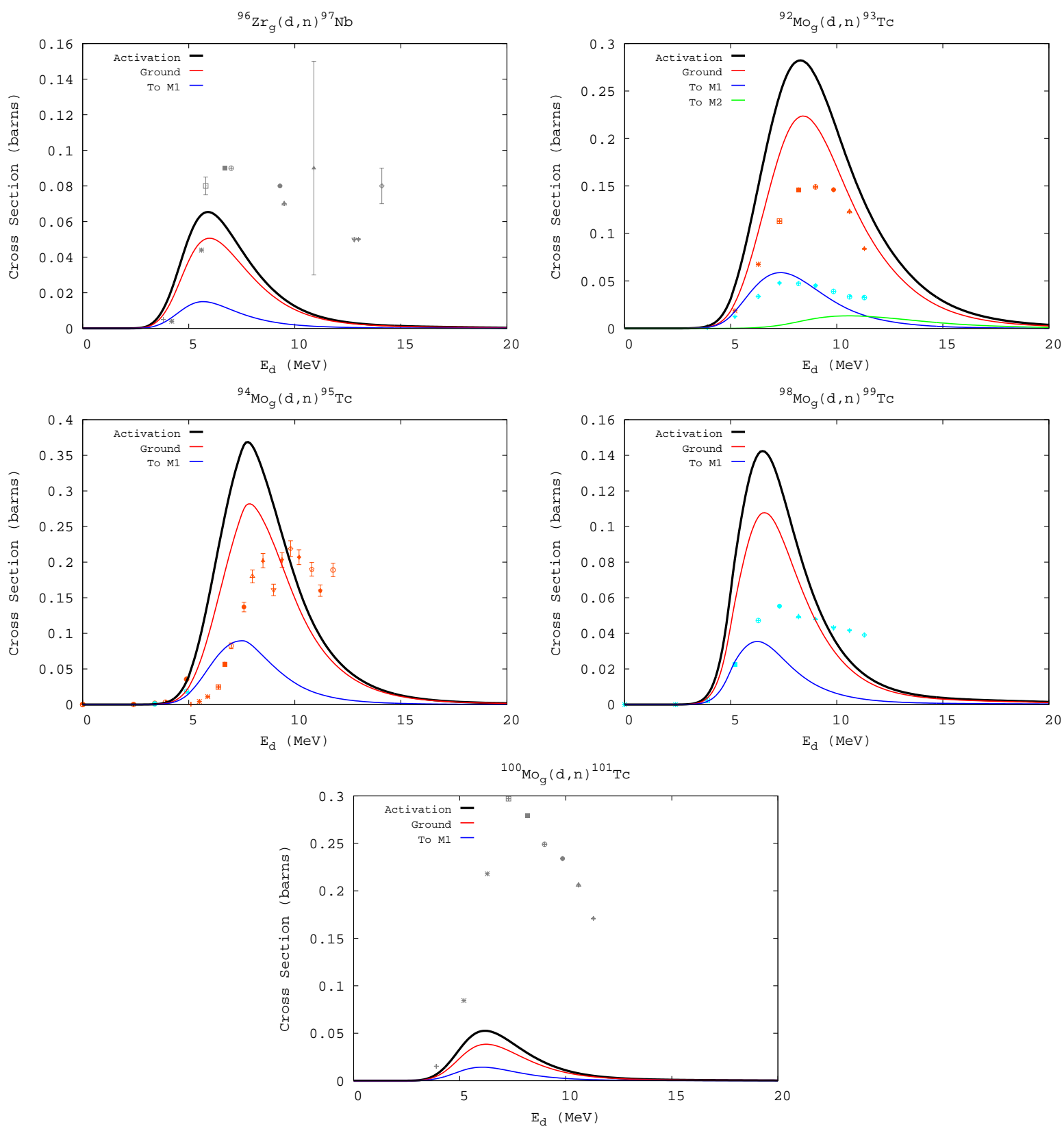

Fig. 40.- Modeled (d,n) cross sections compared to measurement. The data is taken from (EXFOR 2006). The black, red, and blue solid lines represent our modeled cross sections (total, leading to the ground state, and leading to the first isomer, respectively). The Gery, orange, and light blue data points are measured cross section data (total, ground state, and first isomer). 


\section{C.17. $(d, 2 n)$}
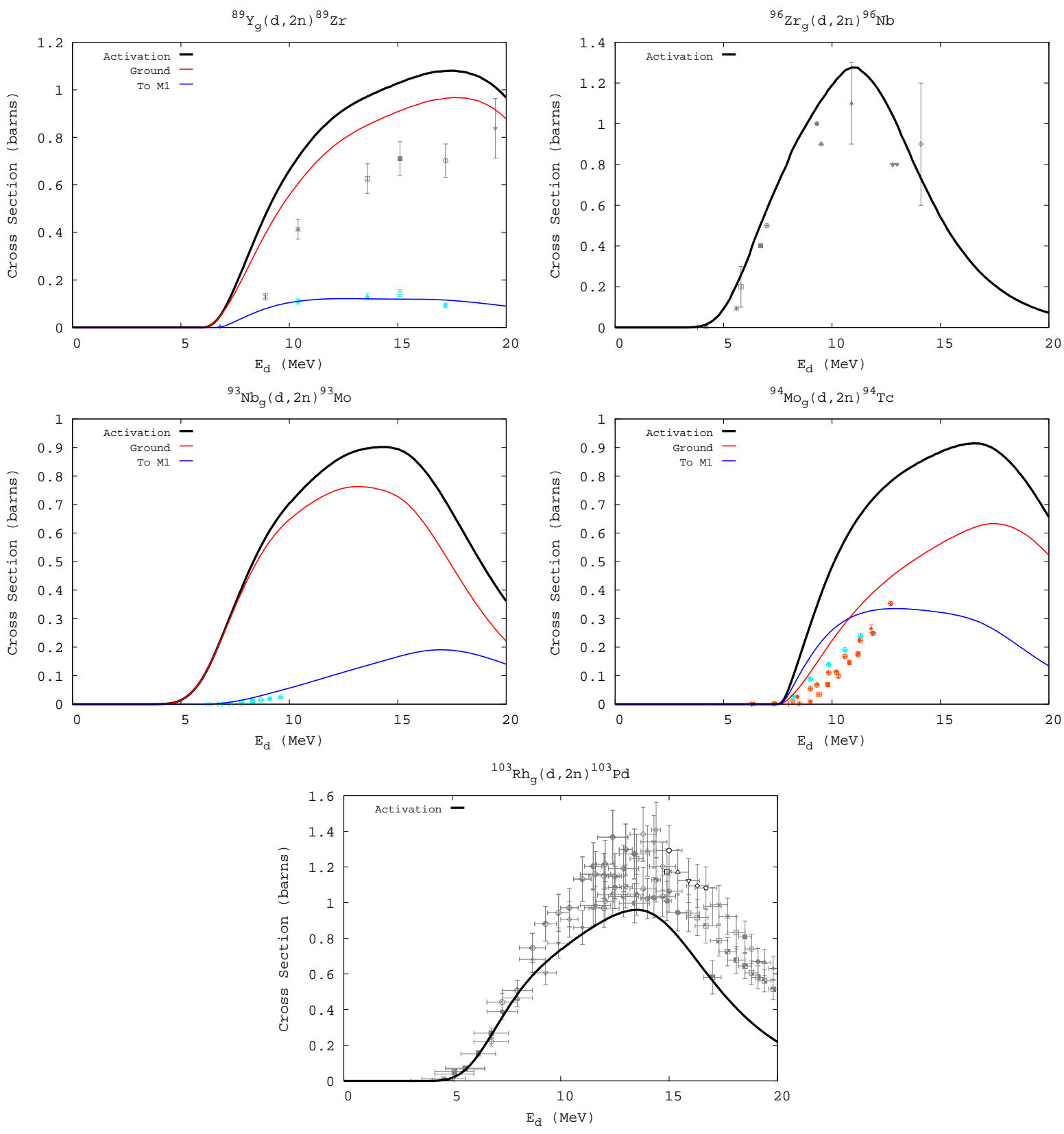

Fig. 41.- Modeled (d,2n) cross sections compared to measurement. The data is taken from (EXFOR 2006). The black, red, and blue solid lines represent our modeled cross sections (total, leading to the ground state, and leading to the first isomer, respectively). The Gery, orange, and light blue data points are measured cross section data (total, ground state, and first isomer). 


\section{C.18. $(d, 3 n)$}

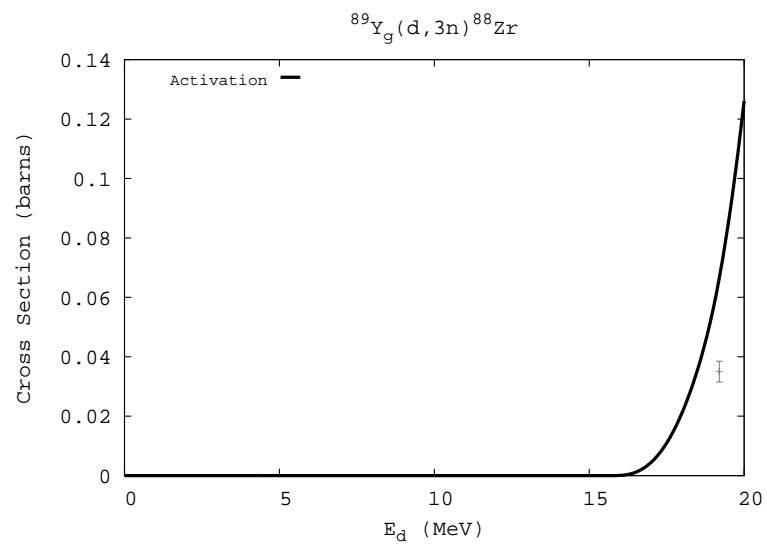

Fig. 42.- Modeled (d,3n) cross sections compared to measurement. The data is taken from (EXFOR 2006). The black, red, and blue solid lines represent our modeled cross sections (total, leading to the ground state, and leading to the first isomer, respectively). The Gery, orange, and light blue data points are measured cross section data (total, ground state, and first isomer). 
C.19. (d,p)

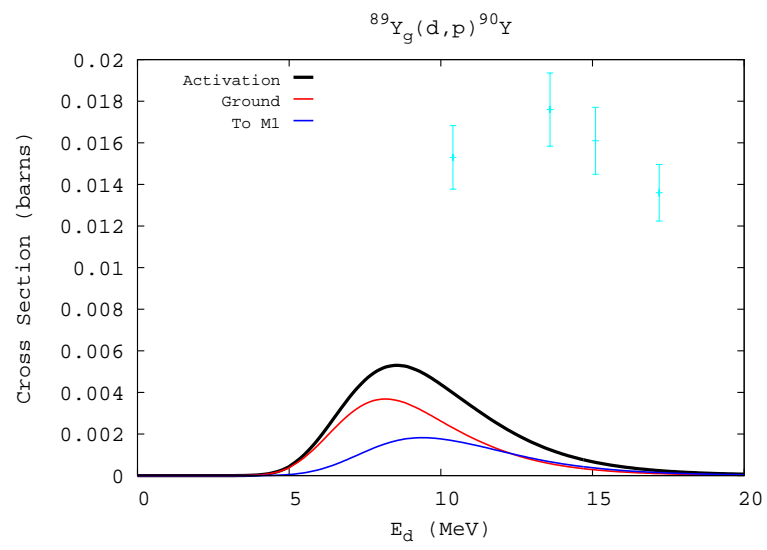

Fig. 43.- Modeled (d,p) cross sections compared to measurement. The data is taken from (EXFOR 2006). The black, red, and blue solid lines represent our modeled cross sections (total, leading to the ground state, and leading to the first isomer, respectively). The Gery, orange, and light blue data points are measured cross section data (total, ground state, and first isomer). 
D. Activation Cross Sections by Target 

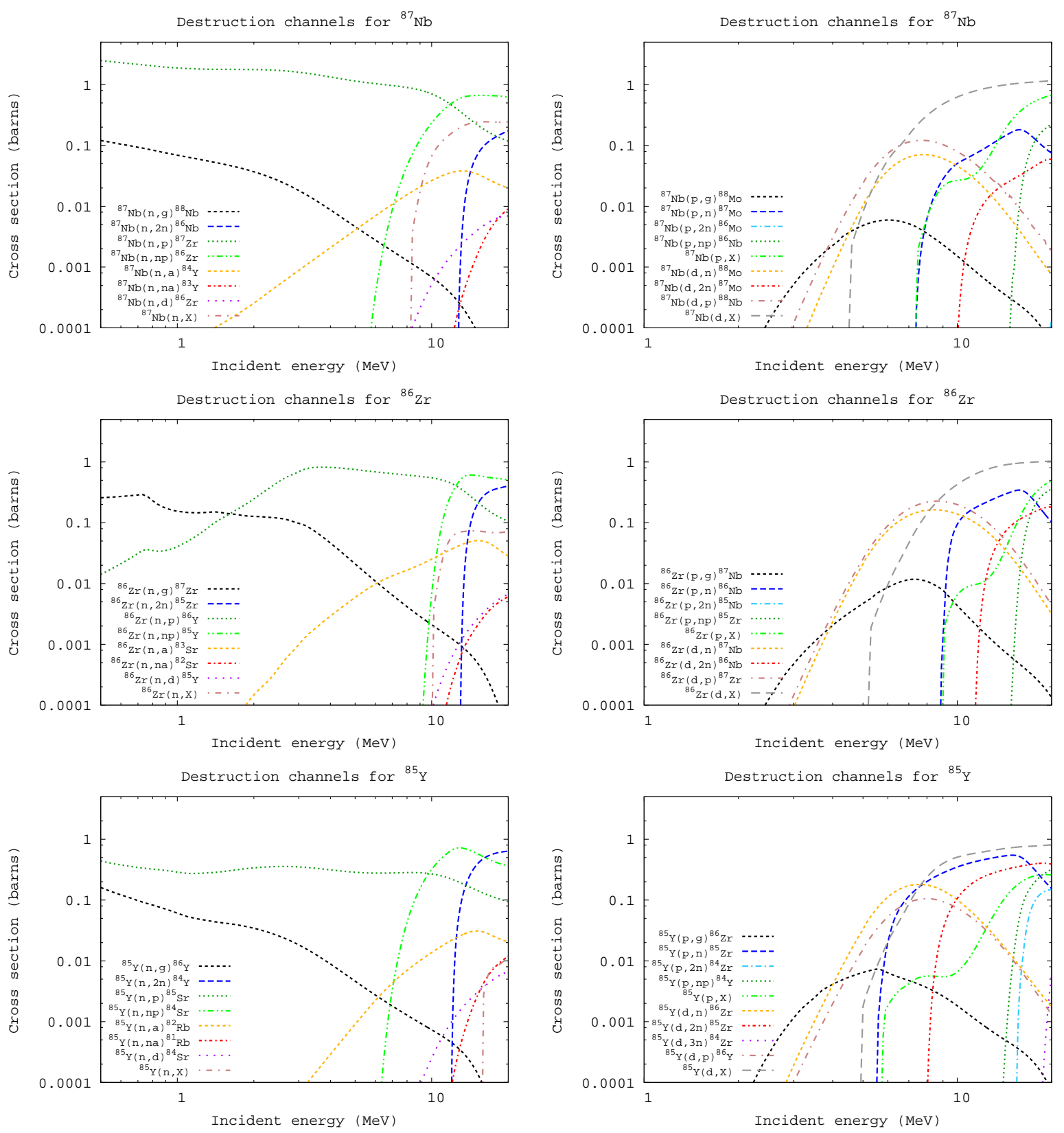

Fig. 44.- Activation cross sections for $\mathrm{N}=46$ ground state targets of $\mathrm{Y}, \mathrm{Zr}$, and $\mathrm{Nb}$. 

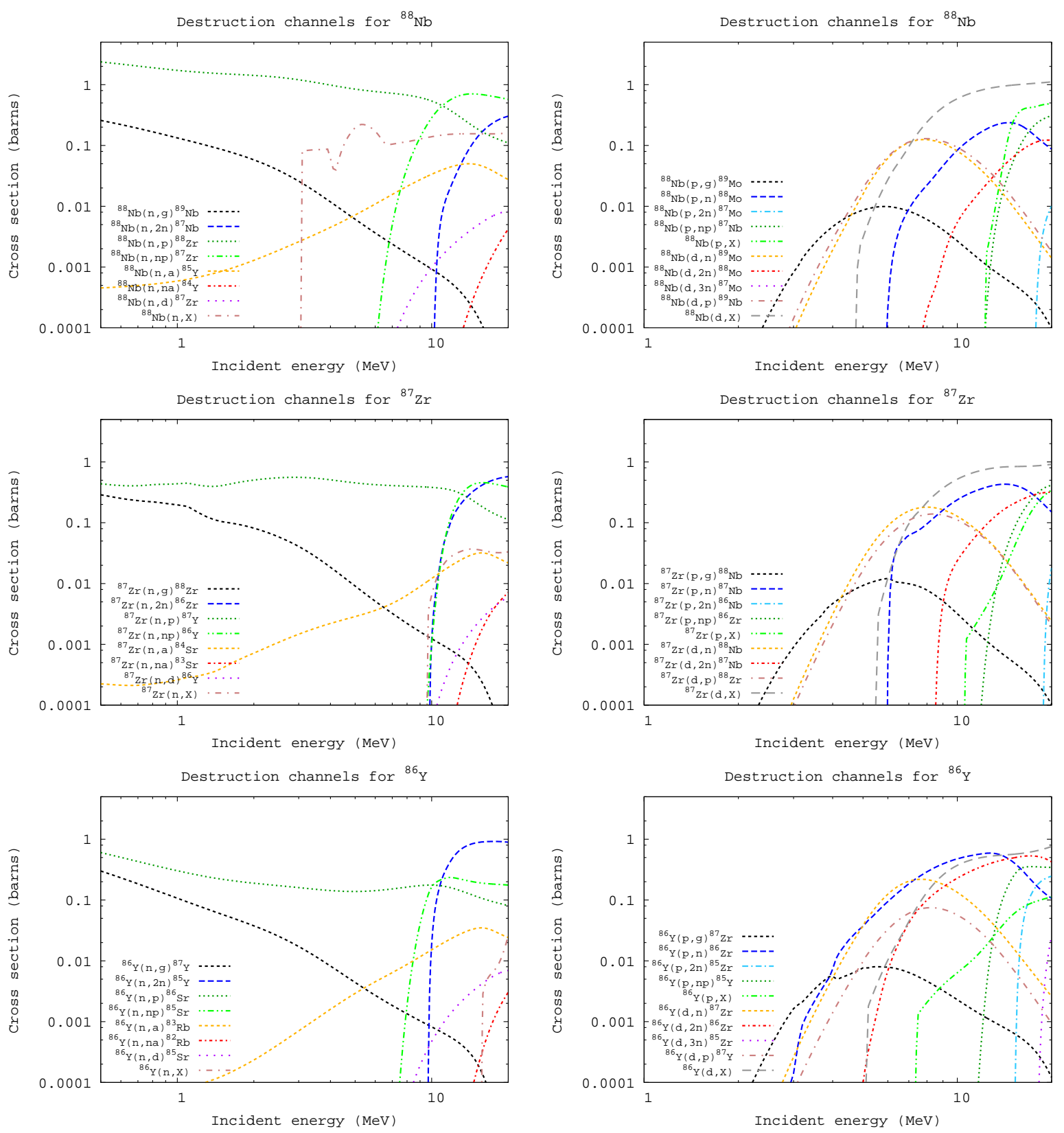

Fig. 45.- Activation cross sections for $\mathrm{N}=47$ ground state targets of $\mathrm{Y}, \mathrm{Zr}$, and $\mathrm{Nb}$. 

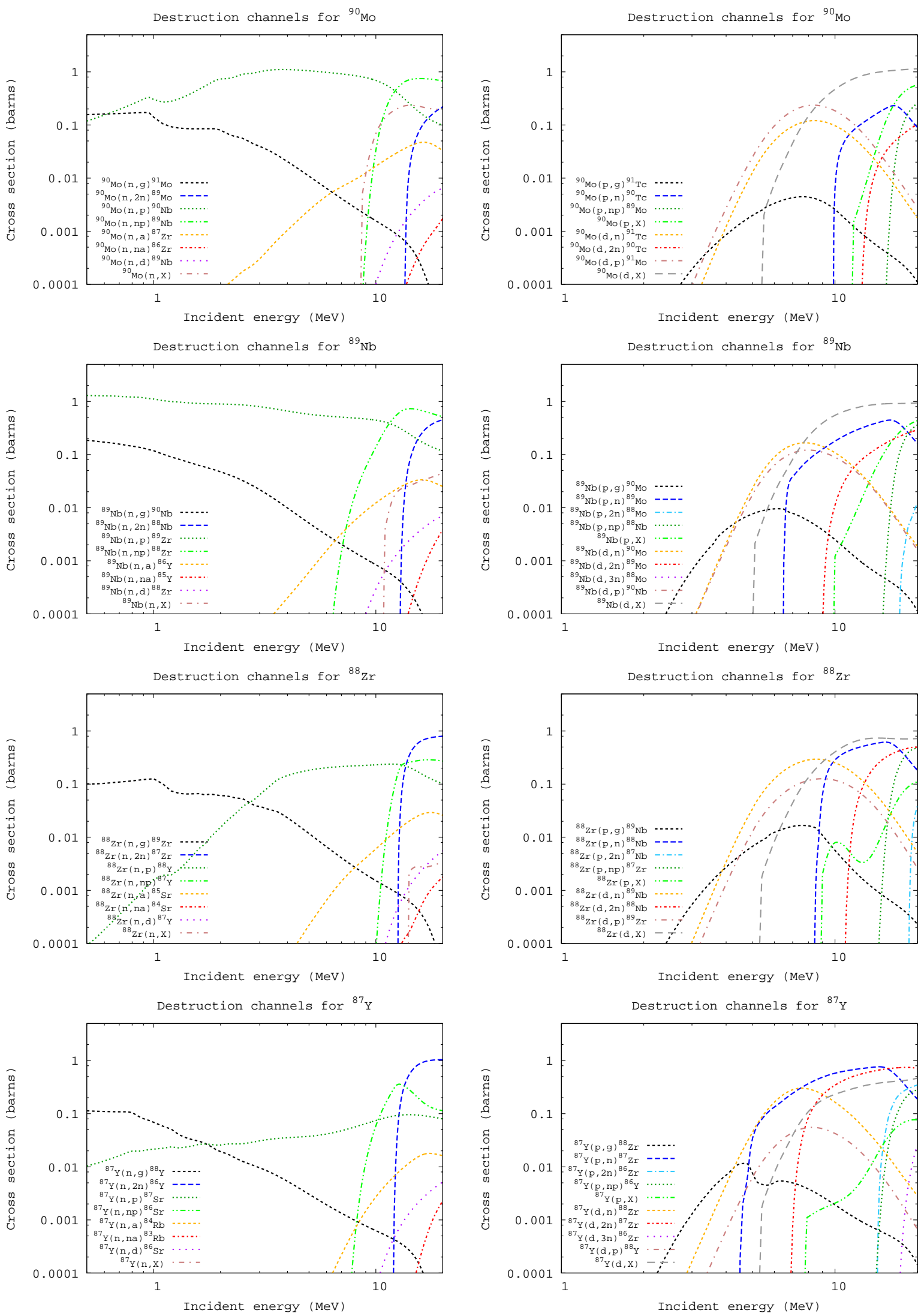

Fig. 46.- Activation cross sections for $\mathrm{N}=48$ ground state targets of $\mathrm{Y}, \mathrm{Zr}, \mathrm{Nb}$, and Mo. 

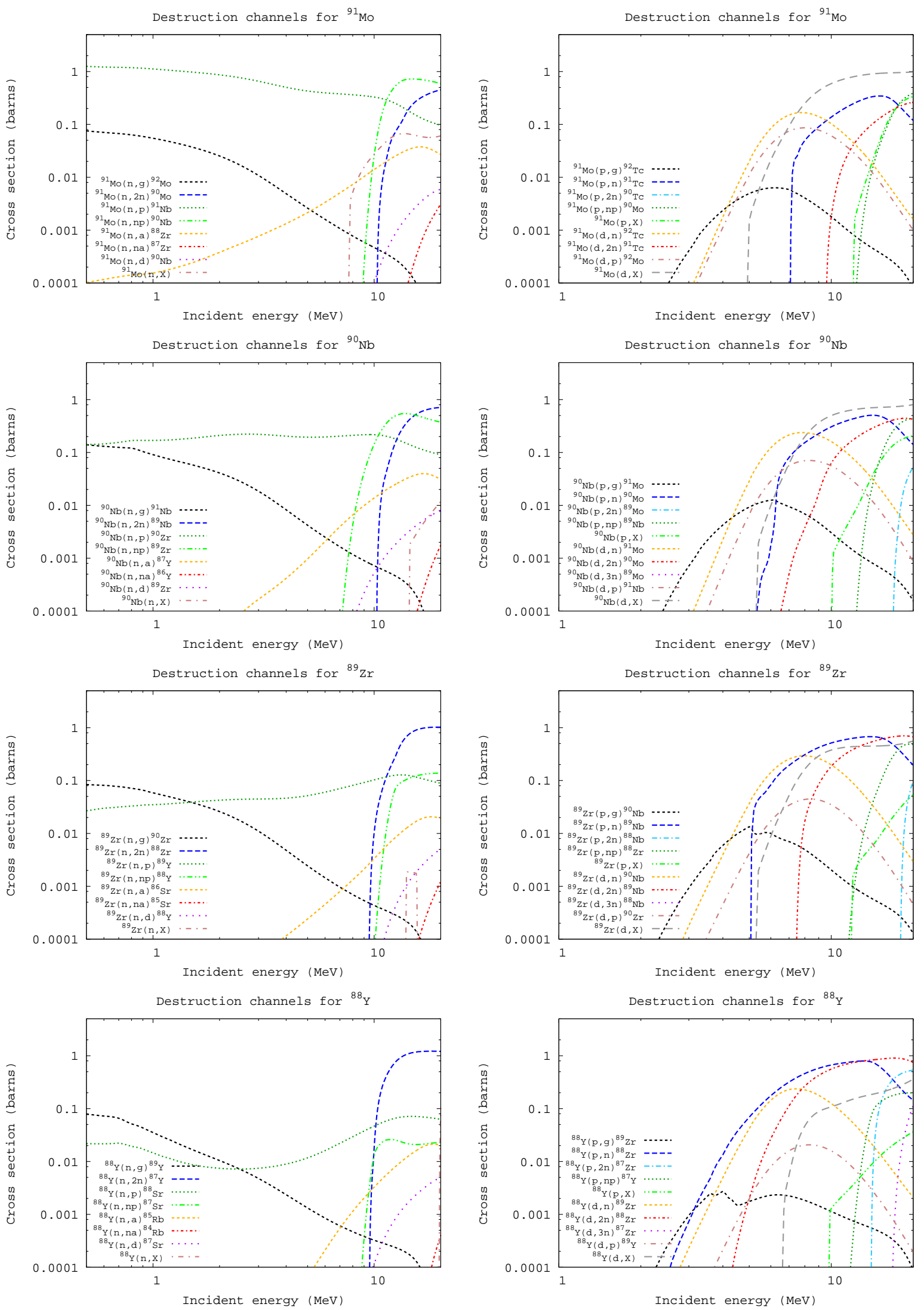

Fig. 47.- Activation cross sections for $\mathrm{N}=49$ ground state targets of $\mathrm{Y}, \mathrm{Zr}, \mathrm{Nb}$, and Mo. 

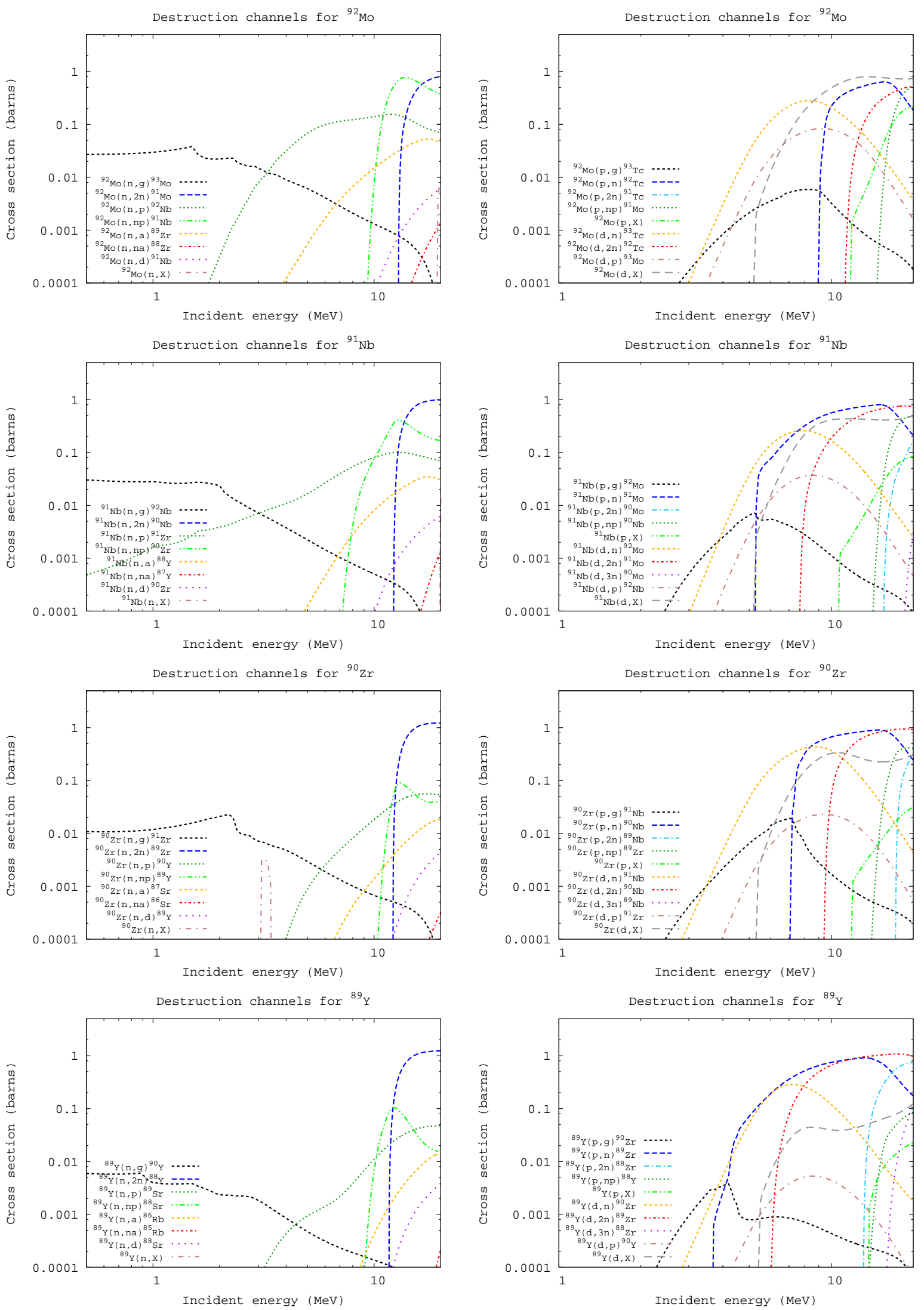

Fig. 48.- Activation cross sections for $\mathrm{N}=50$ ground state targets of $\mathrm{Y}, \mathrm{Zr}, \mathrm{Nb}$, and Mo. 

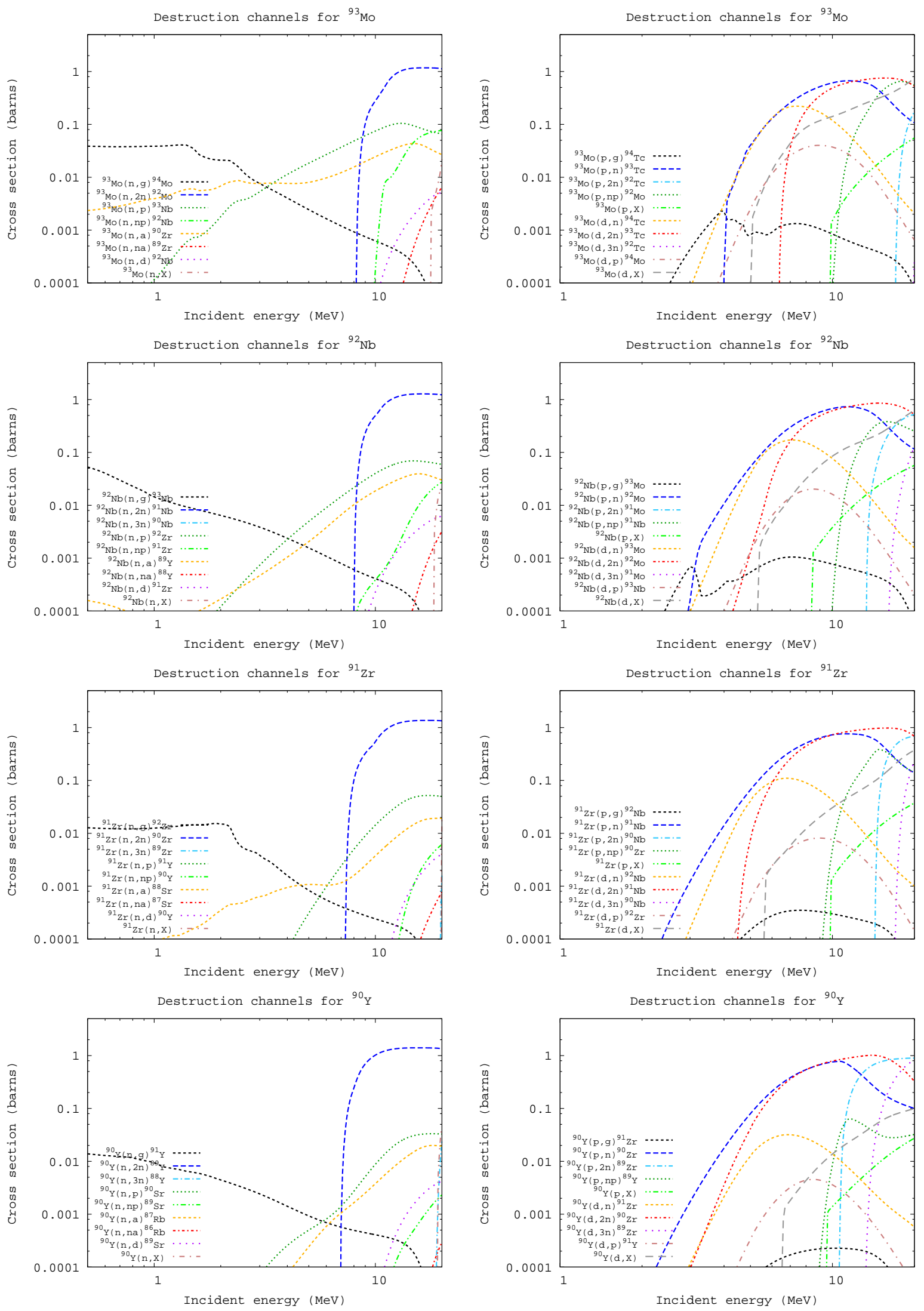

Fig. 49.- Activation cross sections for $\mathrm{N}=51$ ground state targets of $\mathrm{Y}, \mathrm{Zr}$, Nb, and Mo. 

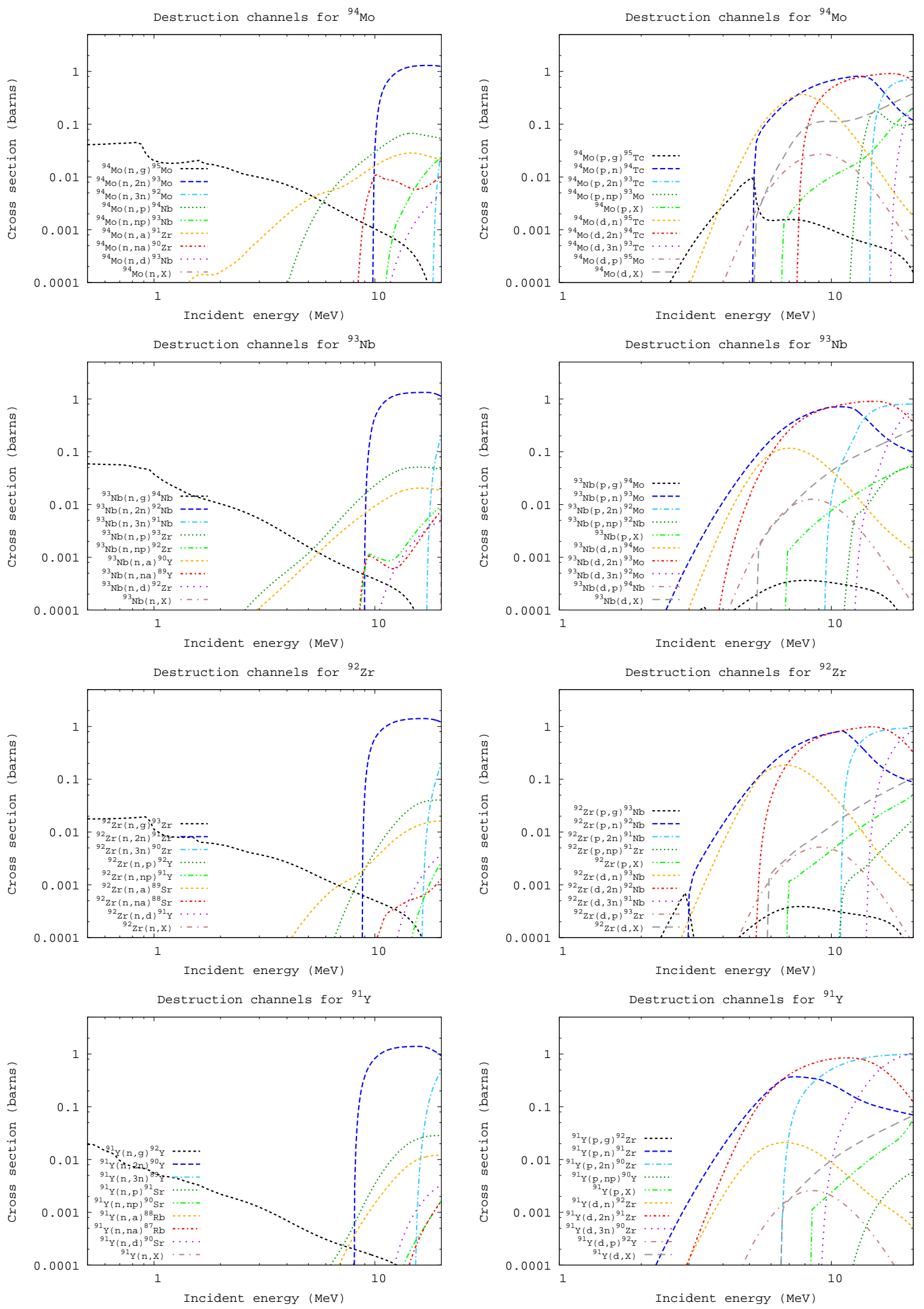

Fig. 50.- Activation cross sections for $\mathrm{N}=52$ ground state targets of $\mathrm{Y}, \mathrm{Zr}, \mathrm{Nb}$, and Mo. 

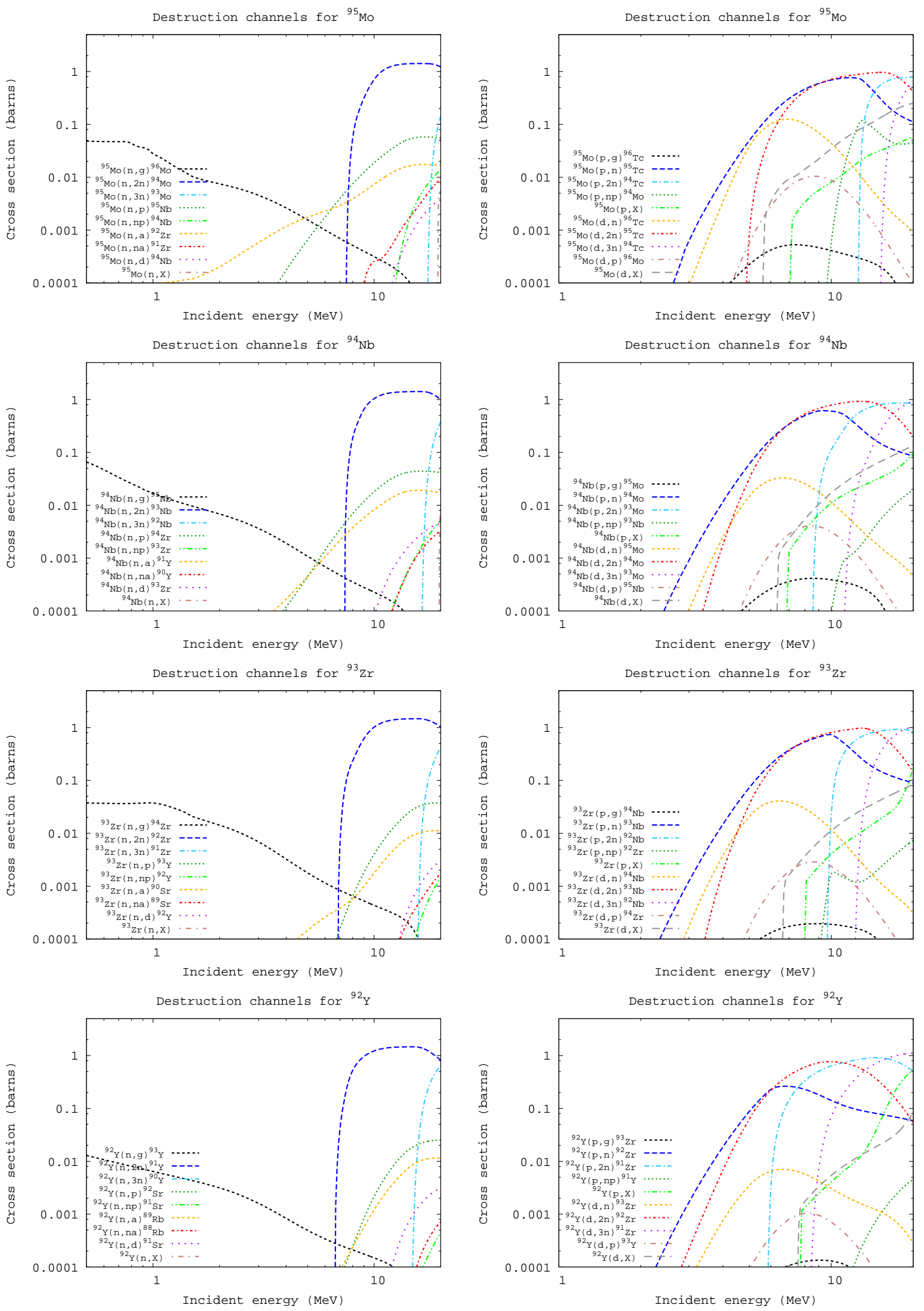

Fig. 51.- Activation cross sections for $\mathrm{N}=53$ ground state targets of $\mathrm{Y}, \mathrm{Zr}, \mathrm{Nb}$, and Mo. 

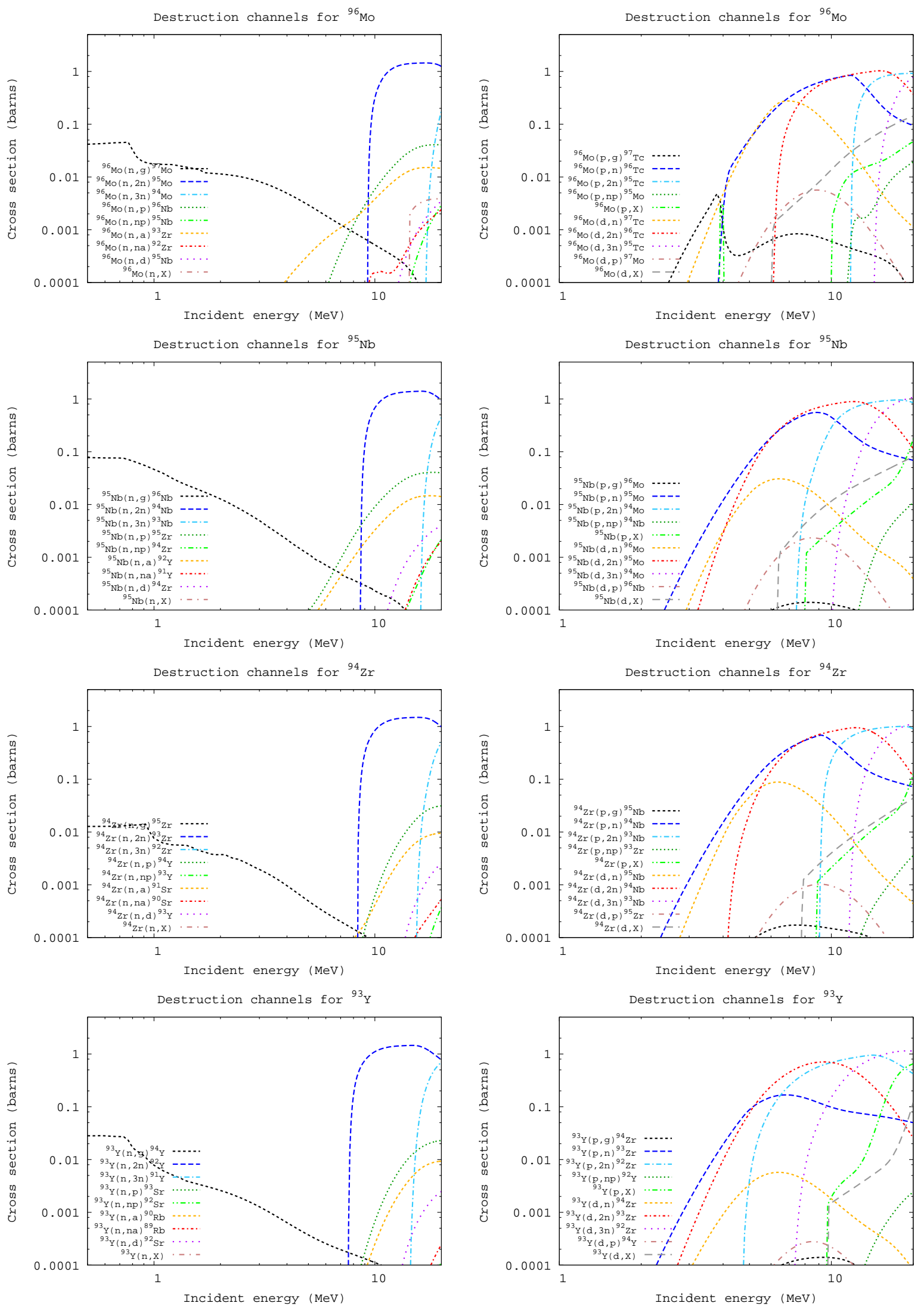

Fig. 52.- Activation cross sections for $\mathrm{N}=54$ ground state targets of $\mathrm{Y}, \mathrm{Zr}, \mathrm{Nb}$, and Mo. 

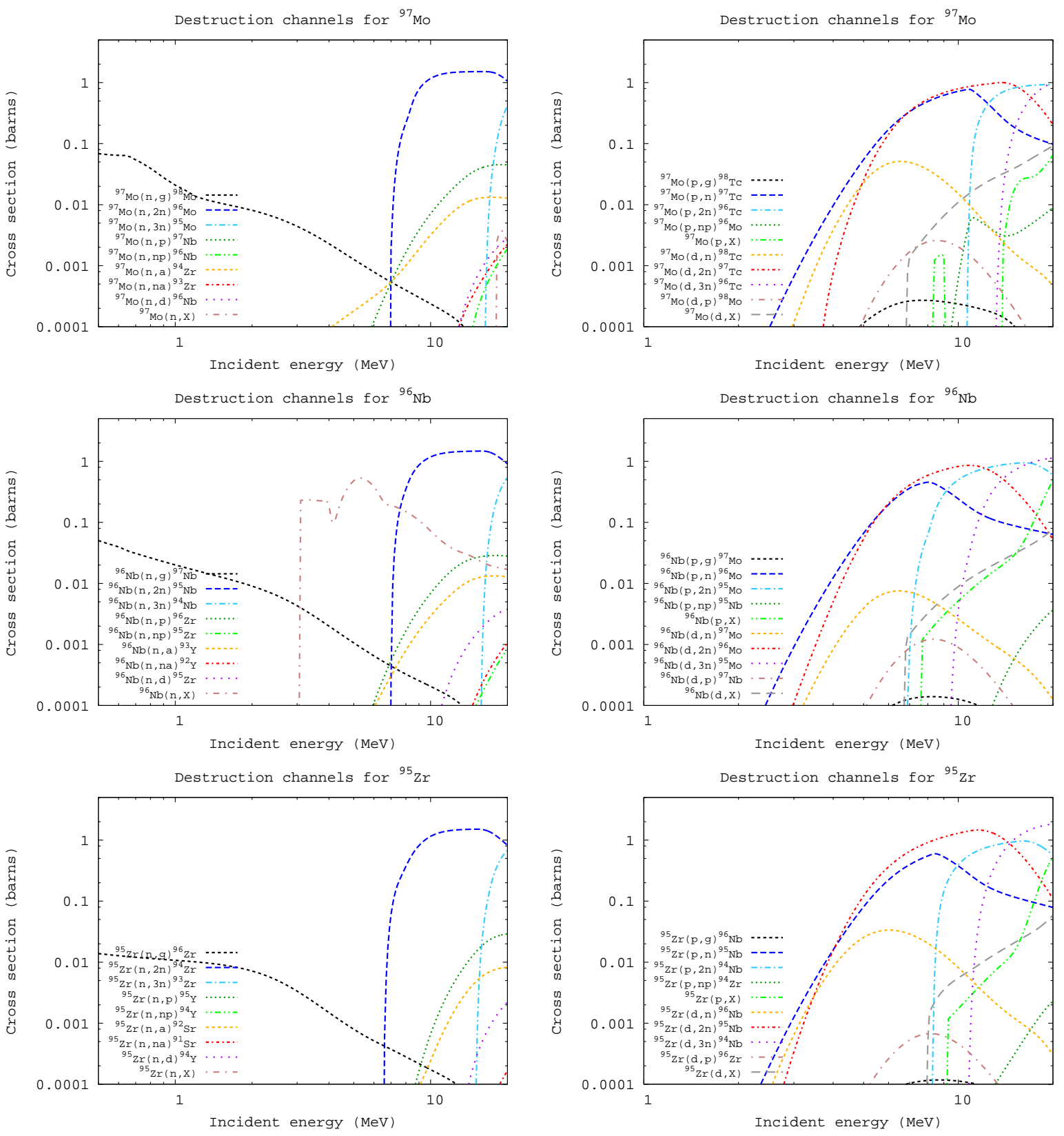

Fig. 53.- Activation cross sections for $\mathrm{N}=55$ ground state targets of $\mathrm{Zr}, \mathrm{Nb}$, and Mo. 

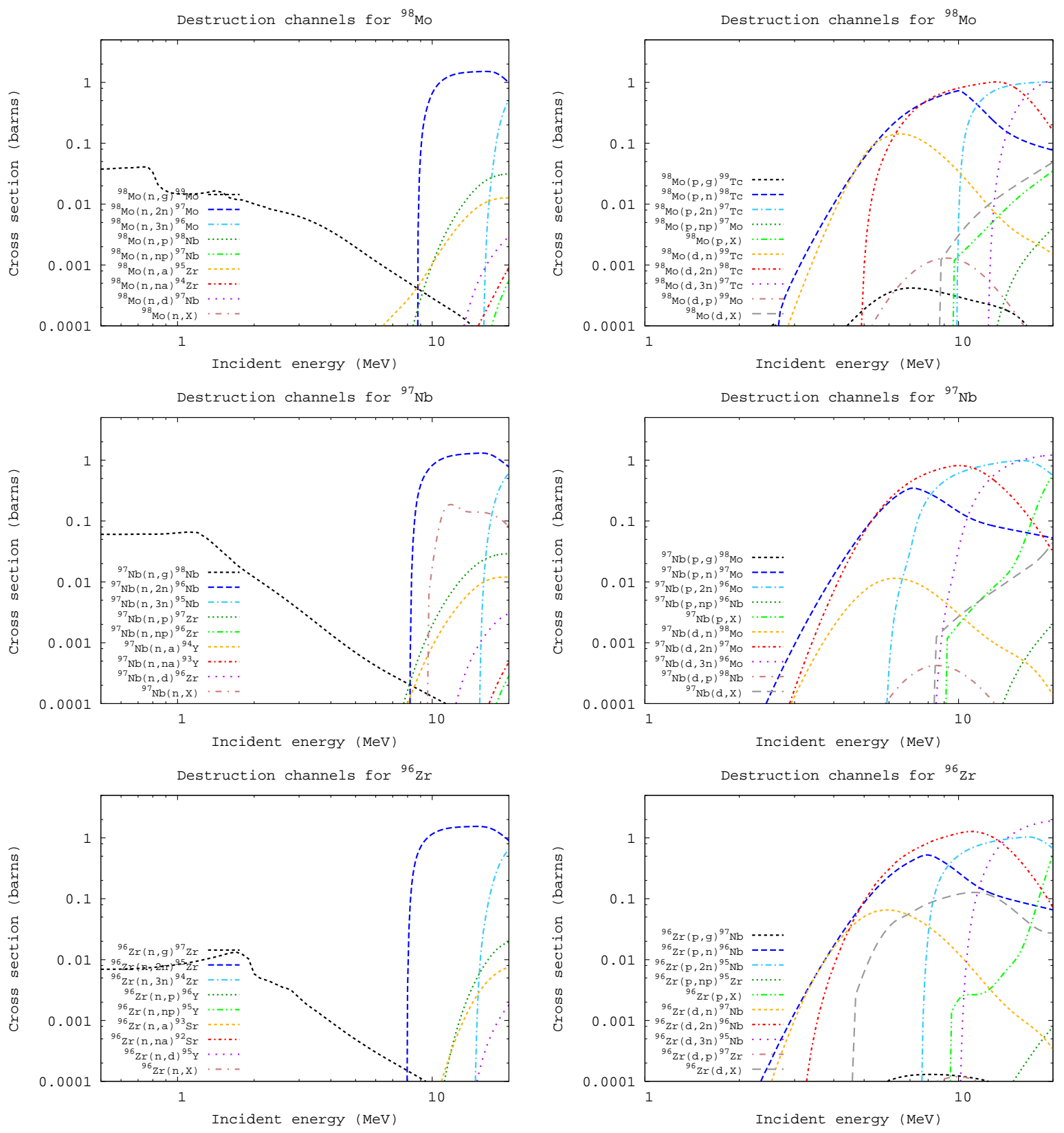

Fig. 54.- Activation cross sections for $\mathrm{N}=56$ ground state targets of $\mathrm{Zr}, \mathrm{Nb}$, and Mo. 